\title{
Structure Elucidation and Absolute Configuration Determination of Nortriterpenoids from Picramnia glazioviana
}

Leila Gimenes, ${ }^{\dagger,}$ Liany Luna-Dulcey, ${ }^{\S}$ Joao M. Batista Jr., ${ }^{\|}$Fernando M. dos Santos Jr., Cecília P. Popolin, ${ }^{\S}$ Marcia R. Cominetti, ${ }^{\S}$ Joao B. Fernandes,,${ }^{* \dagger}$ Dan Staerk ${ }^{*}, \sharp$

${ }^{\dagger}$ Department of Chemistry, Federal University of Sao Carlos (UFSCar), Sao Carlos, SP, Brazil.

${ }^{\S}$ Department of Gerontology, Federal University of Sao Carlos, 13565-905, Sao Carlos, SP, Brazil.

"Institute of Science and Technology, Federal University of Sao Paulo, 12231-280, Sao Jose dos Campos, SP, Brazil.

${ }^{\circ}$ Department of Organic Chemistry, Chemistry Institute, Fluminense Federal University (UFF), Niteroi, RJ, Brazil.

${ }^{\dagger}$ Department of Drug Design and Pharmacology, Faculty of Health and Medical Sciences, University of Copenhagen, Universitetsparken 2, DK-2100 Copenhagen, Denmark. 


\section{TABLE OF CONTENT}

Figure S1. Chromatographic trace at $254 \mathrm{~nm}$ from HPLC-PDA-HRMS-SPE-NMR analysis ........................... 8

Figure S2. ${ }^{1} \mathrm{H}$ NMR spectra obtained in the HPLC-PDA-HRMS-SPE-NMR mode .......................................... 8

Figure S3. Direct correlation of trapped peaks 1, 2, 4, 6-10,14, 15 and 17-21 as well as additional peaks 3, 5,

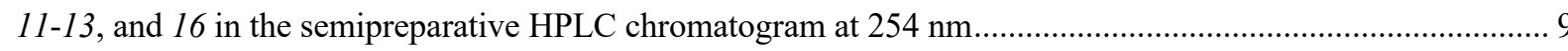

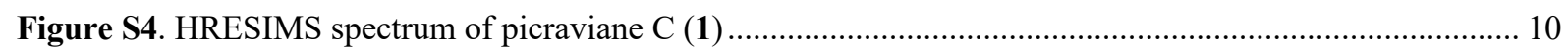

Figure S5. Mass fragmentation mechanism proposed for compounds 2 and 3...................................... 10

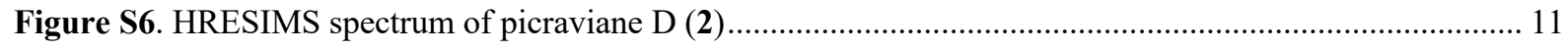

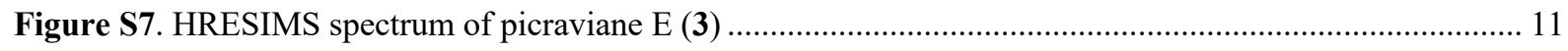

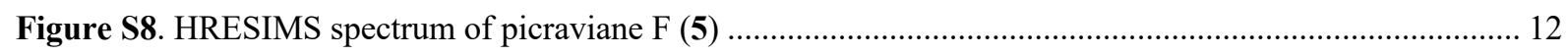

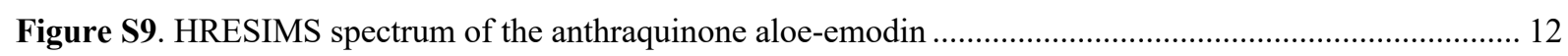

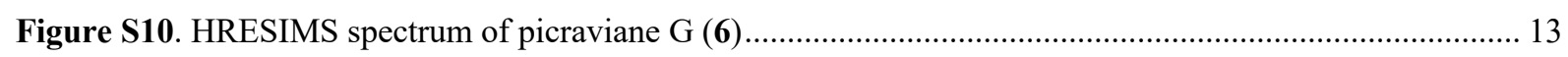

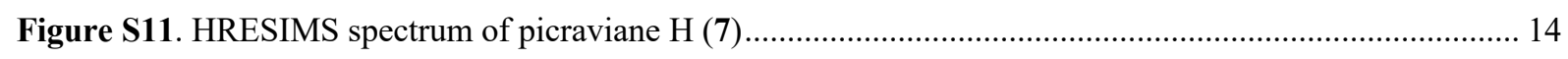

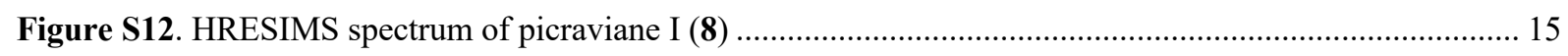

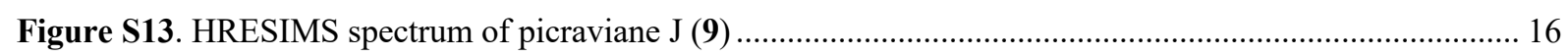

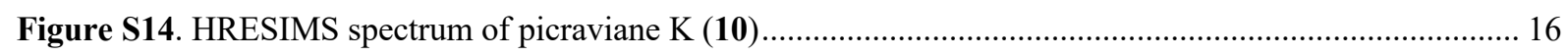

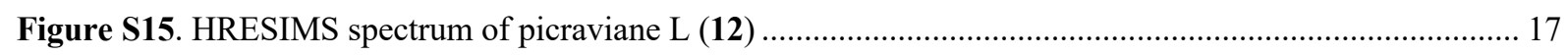

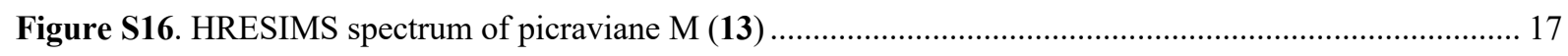

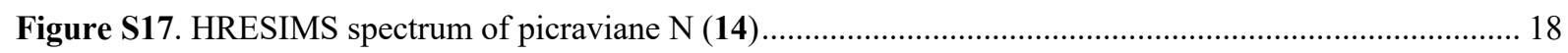

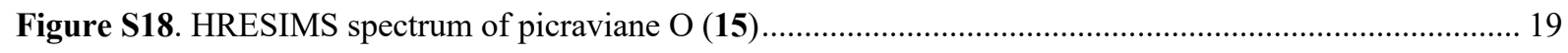

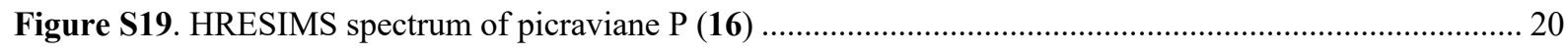

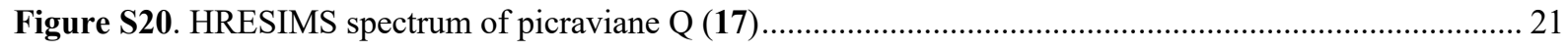

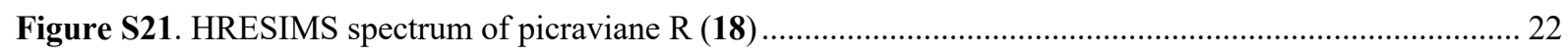

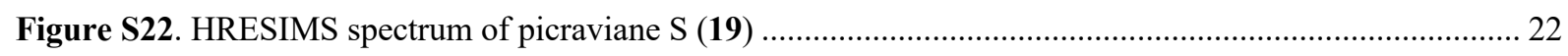

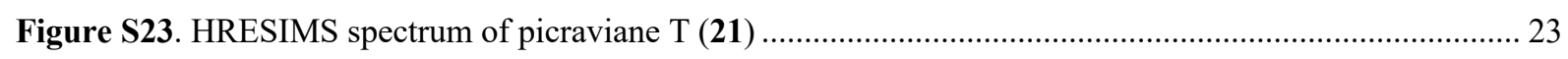

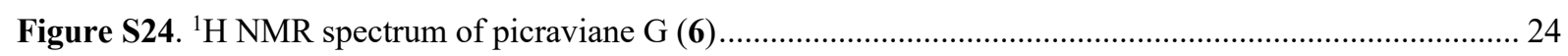

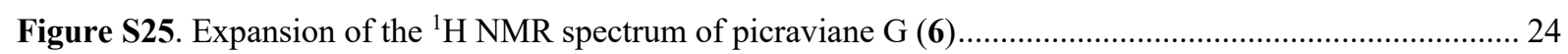

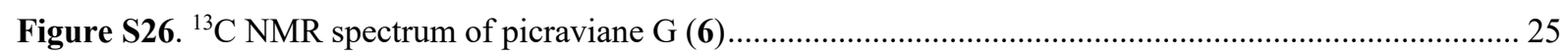

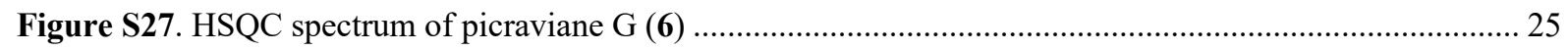

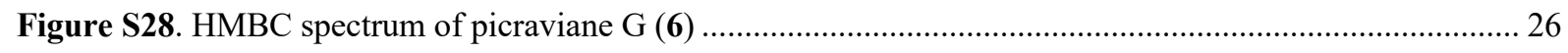

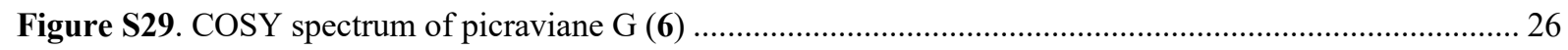

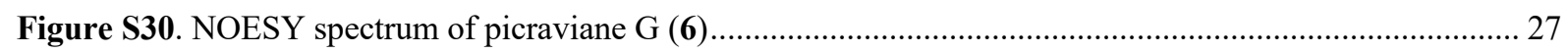

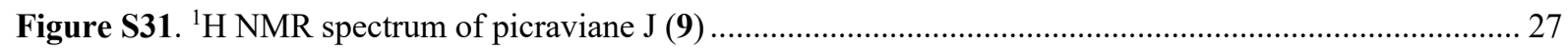

Figure S32. Expansion of the ${ }^{1} \mathrm{H}$ NMR spectrum of picraviane J (9) .......................................................2 28

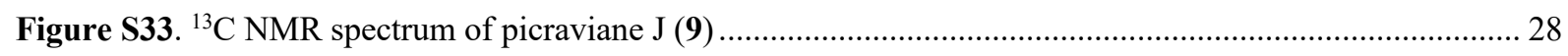

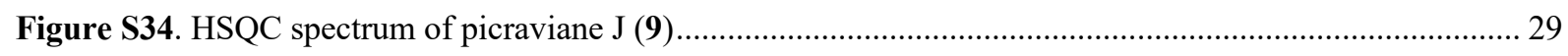


Figure S35. HMBC spectrum of picraviane J (9).

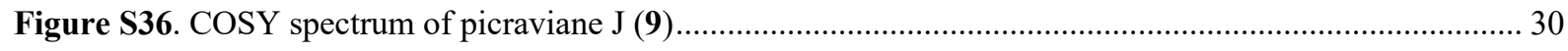

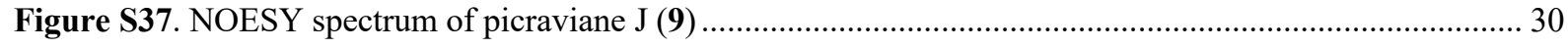

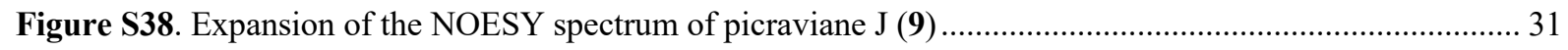

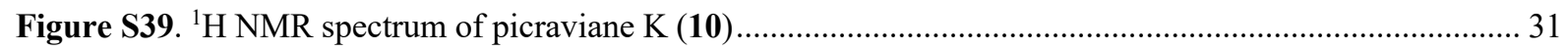

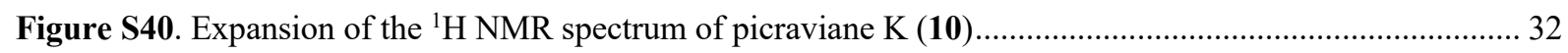

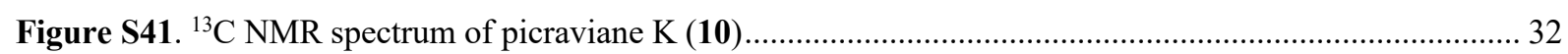

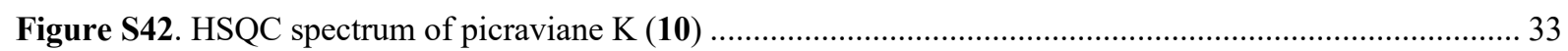

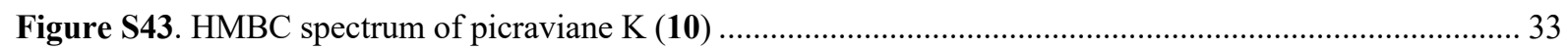

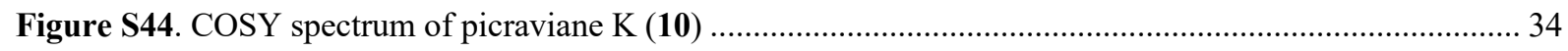

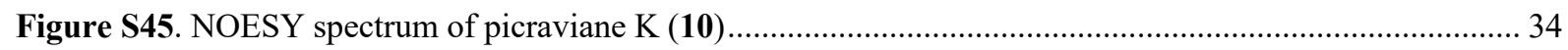

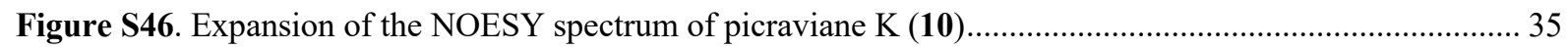

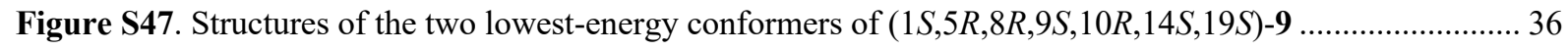

Figure S48. Comparison of the experimental and calculated UV and ECD spectra of

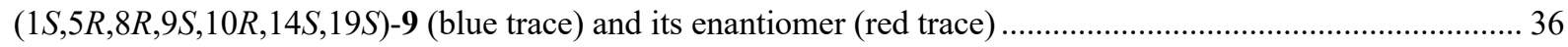

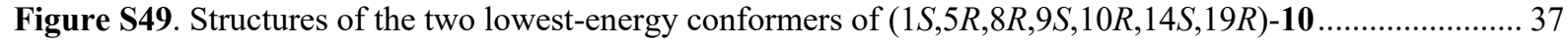

Figure S50. Comparison of the experimental and calculated UV and ECD spectra of $(1 S, 5 R, 8 R, 9 S, 10 R, 14 S, 19 R)-\mathbf{1 0}$ (blue trace) and its enantiomer (red trace) ................................................. 37

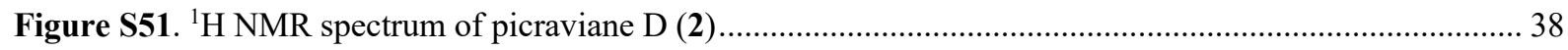

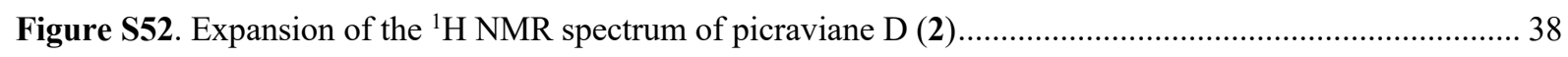

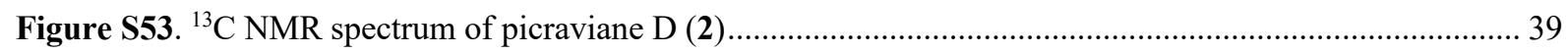

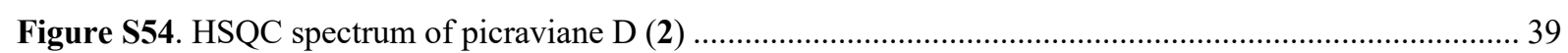

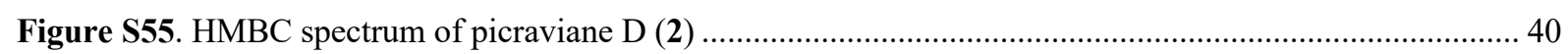

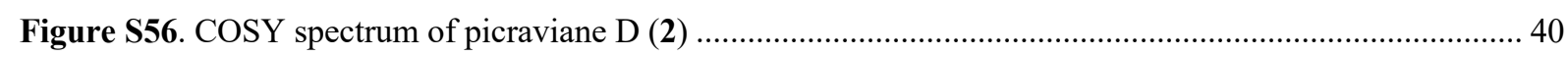

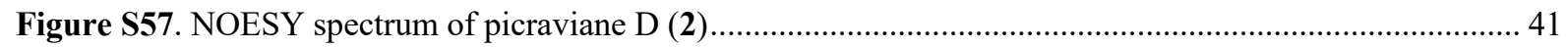

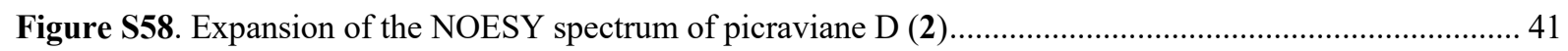

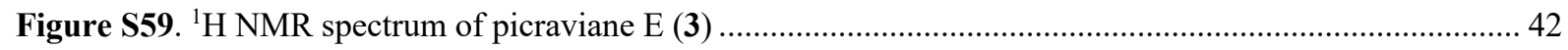

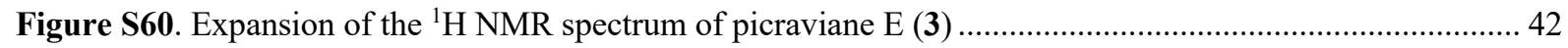

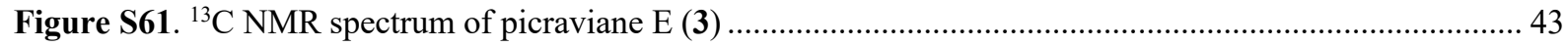

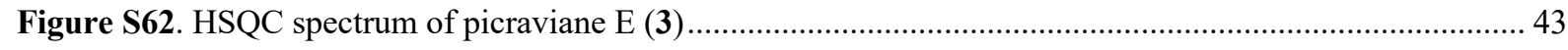

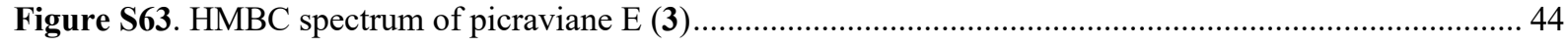

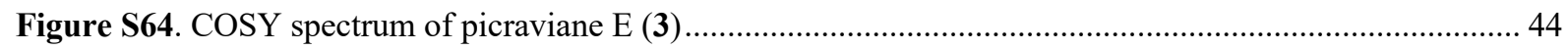

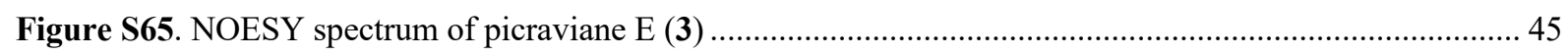

Figure S66. Expansion of the NOESY spectrum of picraviane E (3) .............................................................45

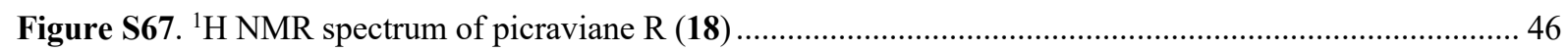

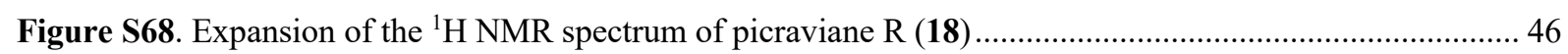

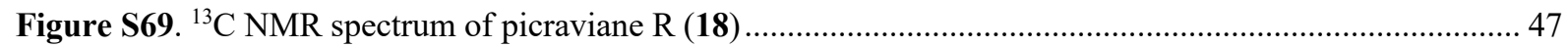




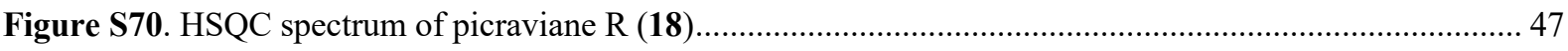

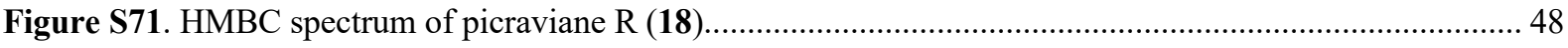

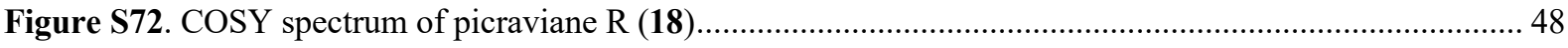

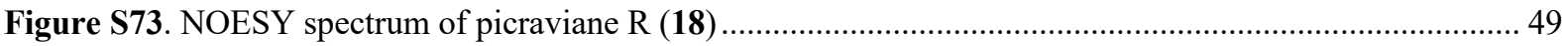

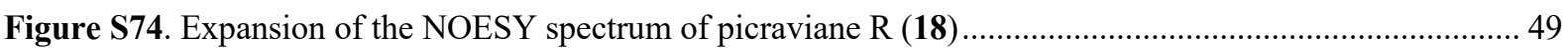

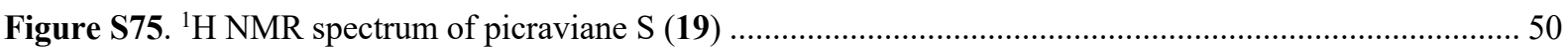

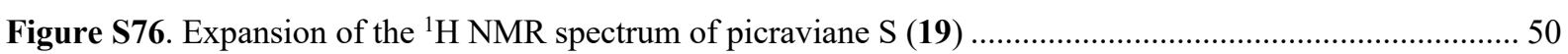

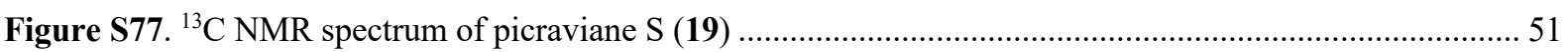

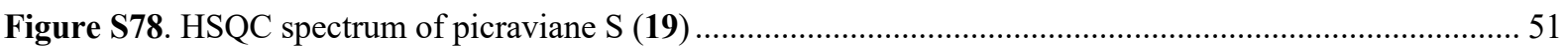

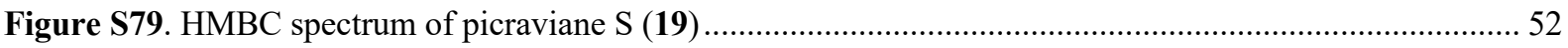

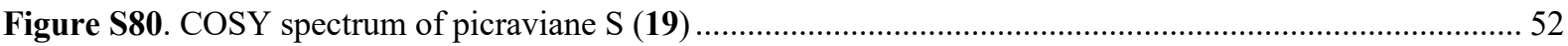

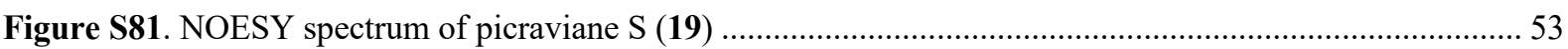

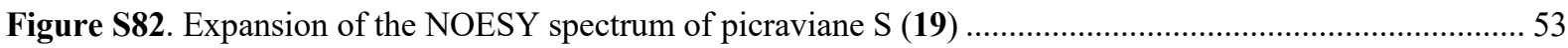

Figure S83. Structures of the two lowest-energy conformers of $(1 S, 5 R, 7 R, 8 R, 9 S, 10 R, 14 R, 19 S)-18 \ldots \ldots \ldots \ldots \ldots . . . .54$

Figure S84. Comparison of the experimental and calculated UV and ECD spectra of $(1 S, 5 R, 7 R, 8 R, 9 S, 10 R, 14 R, 19 S)-\mathbf{1 8}$ (blue trace) and its enantiomer (red trace) ..............................................5 54

Figure S85. Structures of the two lowest-energy conformers of $(1 S, 5 R, 7 R, 8 R, 9 S, 10 R, 14 R, 19 R)-19 \ldots \ldots \ldots \ldots \ldots . . . .55$

Figure S86. Comparison of the experimental and calculated UV and ECD spectra of $(1 S, 5 R, 7 R, 8 R, 9 S, 10 R, 14 R, 19 R)-19$ (blue trace) and its enantiomer (red trace) ............................................ 55

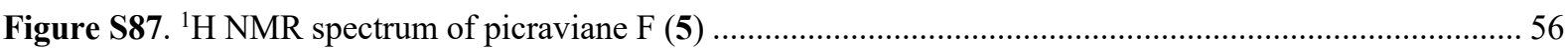

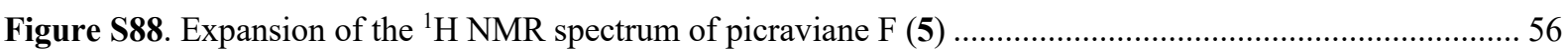

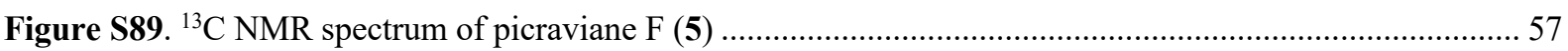

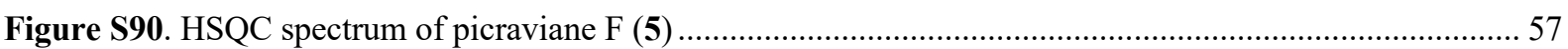

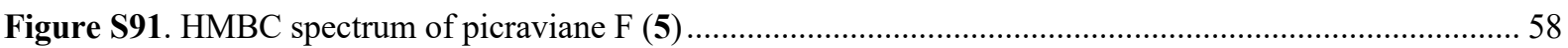

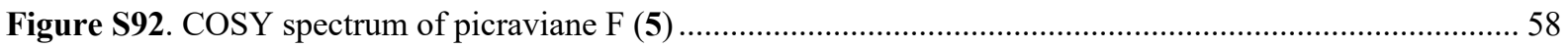

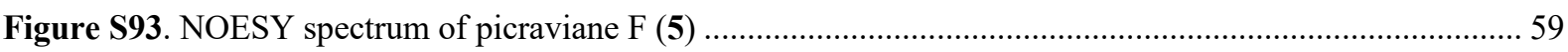

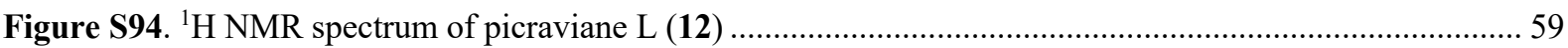

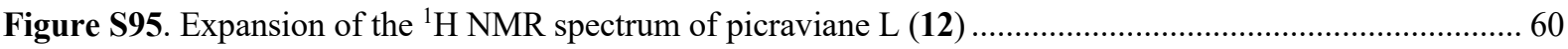

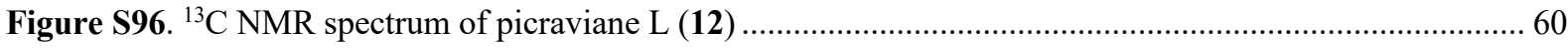

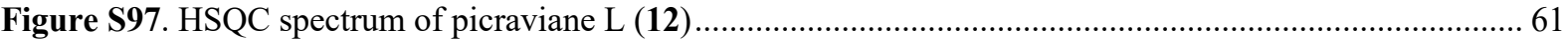

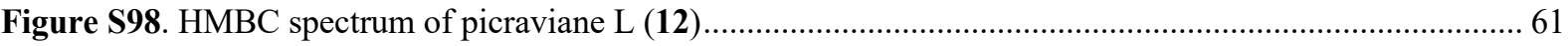

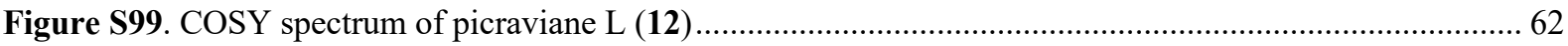

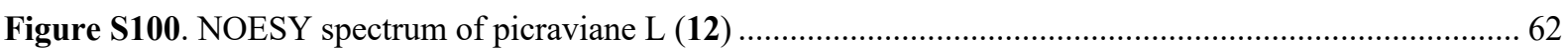

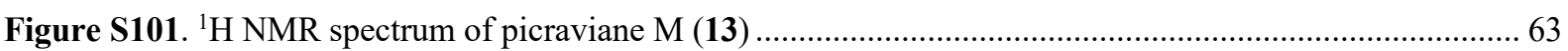

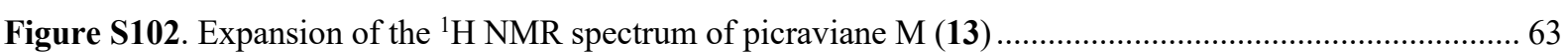

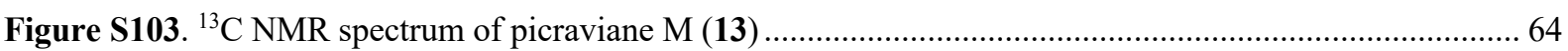

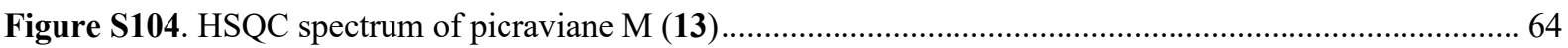




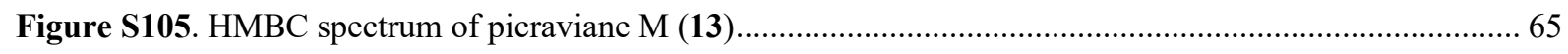

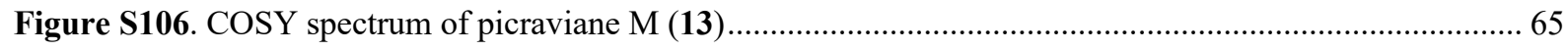

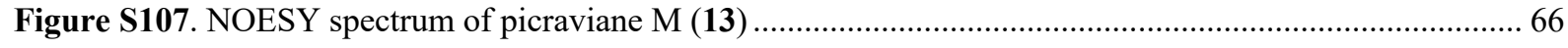

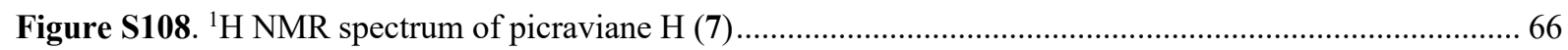

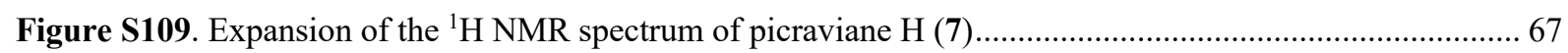

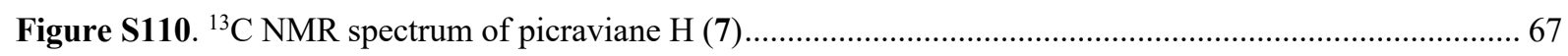

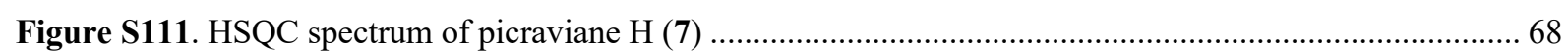

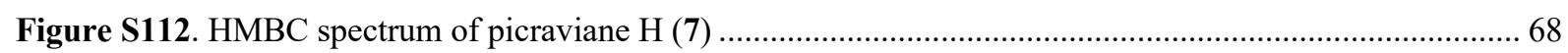

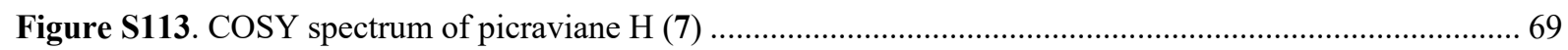

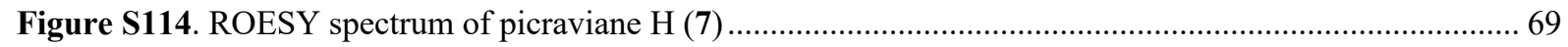

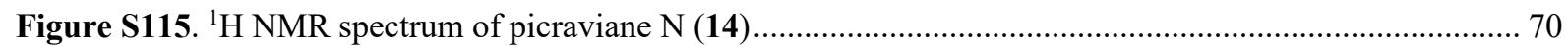

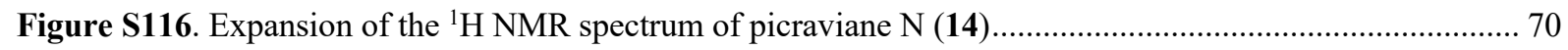

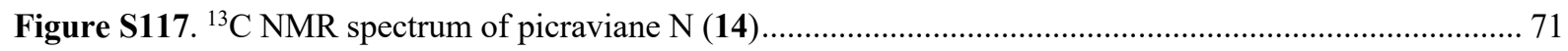

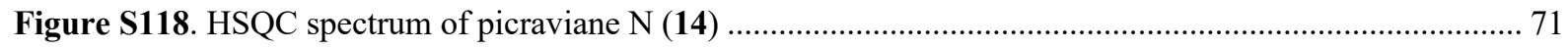

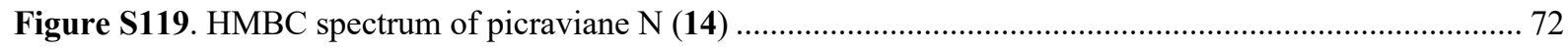

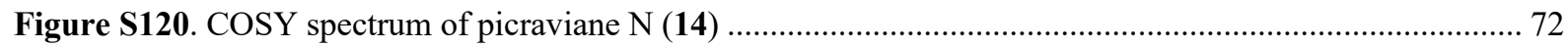

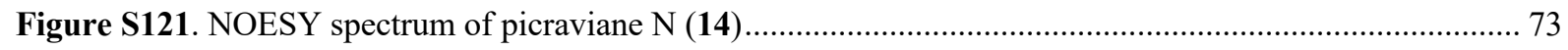

Figure S122. Structures of the five lowest-energy conformers of $(1 S, 5 R, 8 R, 9 S, 10 R, 14 S, 19 S)-7 \ldots \ldots \ldots \ldots \ldots \ldots . . . . . . . . . .74$

Figure S123. Comparison of the experimental and calculated UV and ECD spectra of $(1 S, 5 R, 8 R, 9 S, 10 R, 14 S, 19 S)-7$ (blue trace) and its enantiomer (red trace) .................................................... 75

Figure S124. Structure of lowest-energy conformations and ECD spectra of the $(1 S, 5 R, 8 R, 9 S, 10 R, 14 S, 19 R)$ -

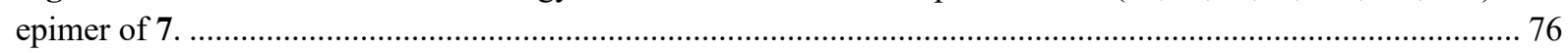

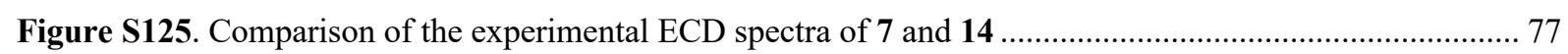

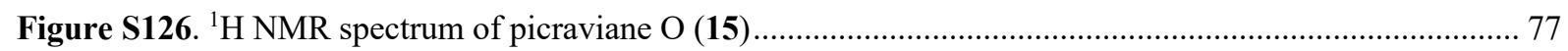

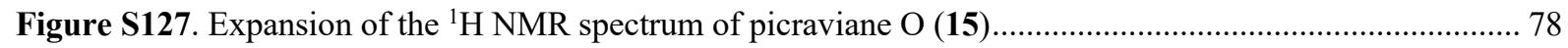

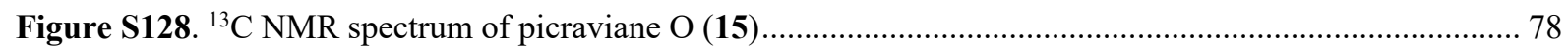

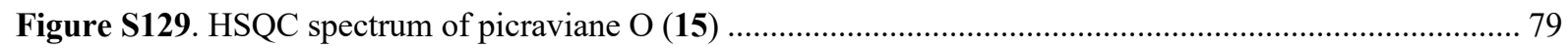

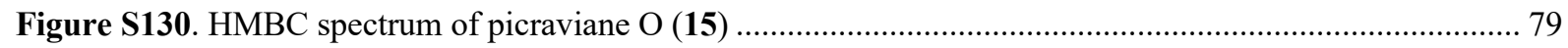

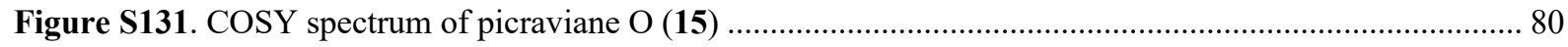

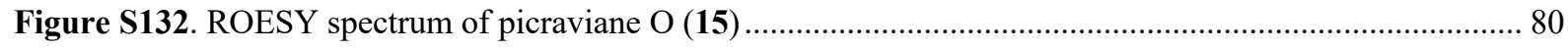

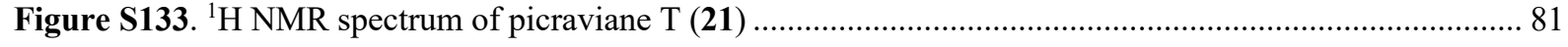

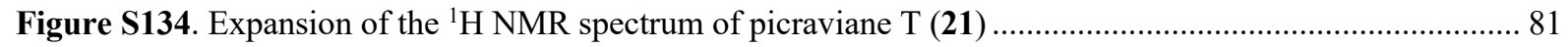

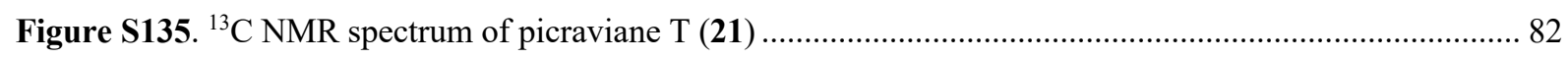

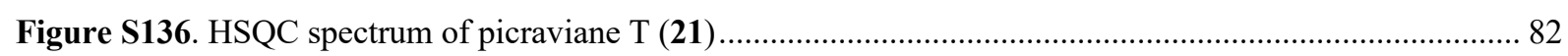

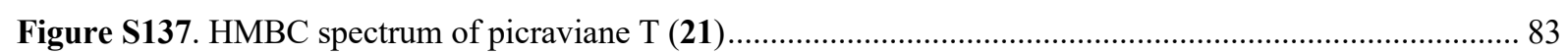

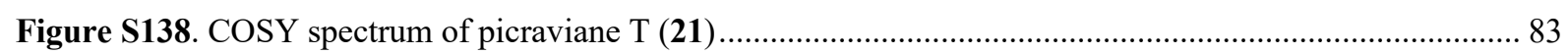

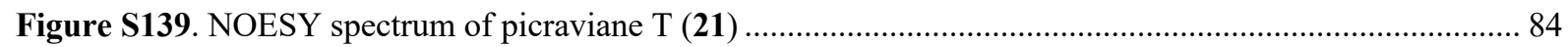


Figure S140. Structures of the three lowest-energy conformers of $(1 S, 5 R, 7 R, 8 R, 9 S, 10 R, 14 R, 19 S)-15$

Figure S141. Comparison of the experimental and calculated UV and ECD spectra of

$(1 S, 5 R, 7 R, 8 R, 9 S, 10 R, 14 R, 19 S)-\mathbf{1 5}$ (blue trace) and its enantiomer (red trace)

Figure S142. Structures of lowest-energy conformations and ECD spectra of $(1 S, 5 R, 7 R, 8 R, 9 S, 10 R, 14 R, 19 R)-15$

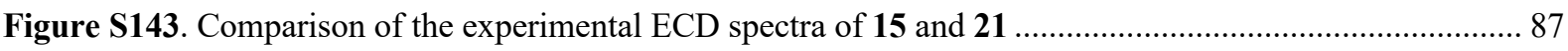

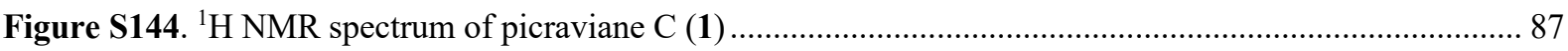

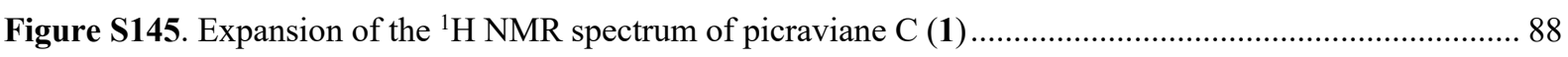

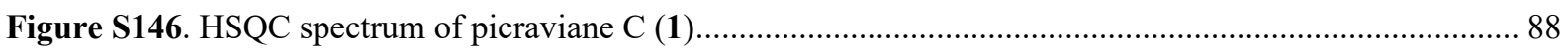

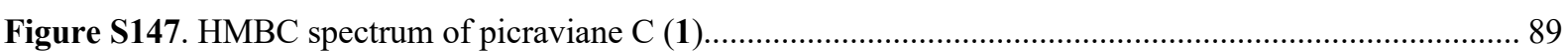

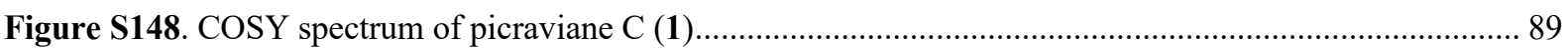

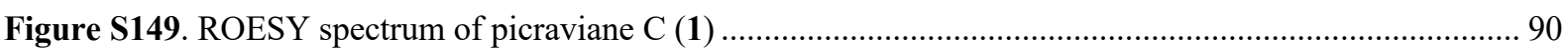

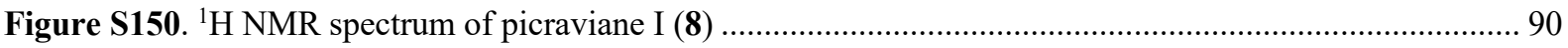

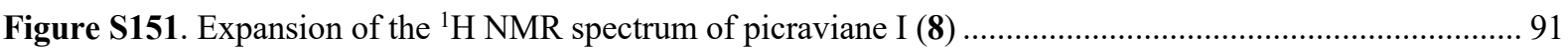

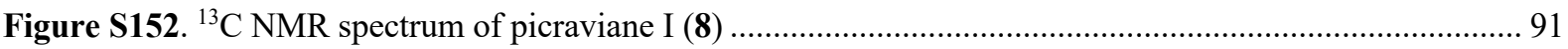

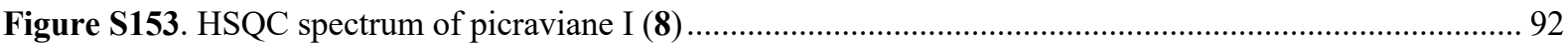

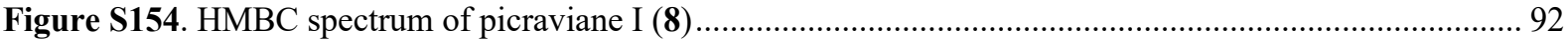

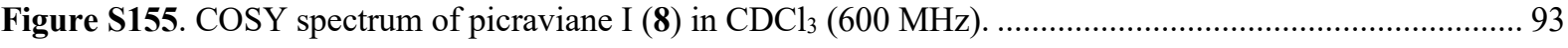

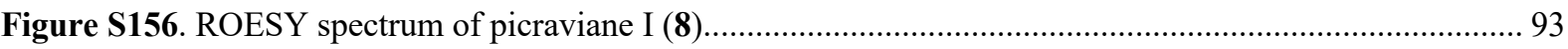

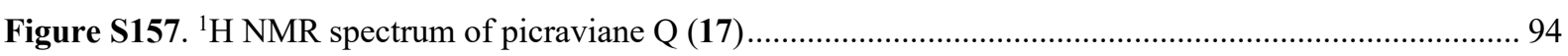

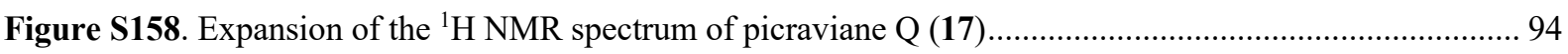

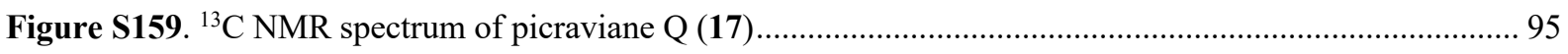

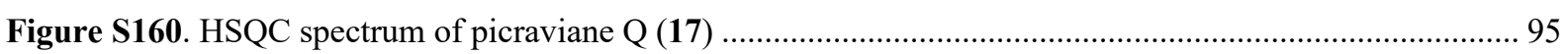

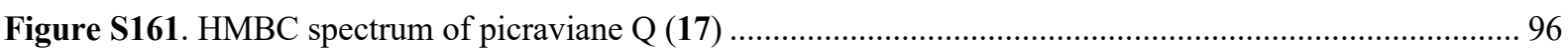

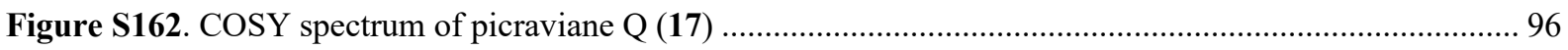

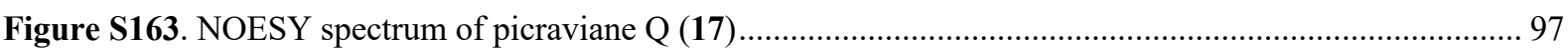

Figure S164. Structures of the five lowest-energy conformers of $(1 S, 5 R, 7 R, 8 R, 9 S, 10 R, 14 R, 19 S)-17 \ldots \ldots \ldots \ldots . . .98$

Figure S165. Comparison of the experimental and calculated UV and ECD spectra of $(1 S, 5 R, 7 R, 8 R, 9 S, 10 R, 14 R, 19 S)-17$ (blue trace) and its enantiomer (red trace) ............................................ 99

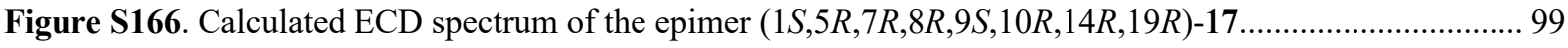

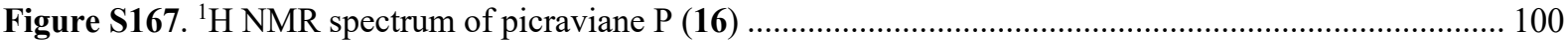

Figure S168. Expansion of the ${ }^{1} \mathrm{H}$ NMR spectrum of picraviane P (16) ............................................... 100

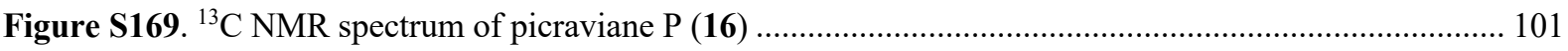

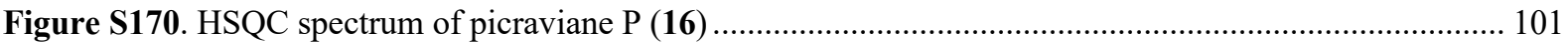

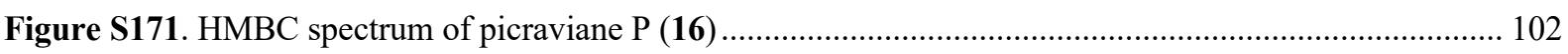

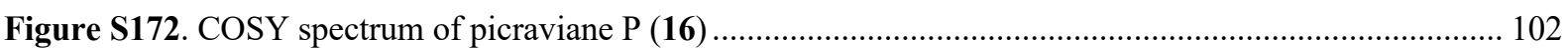

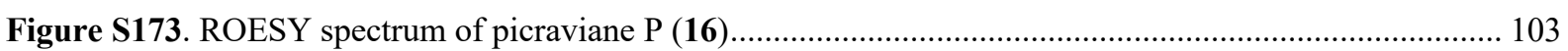


Figure S174. Effect of compounds 6, 10, 14, 16, 17, 18, 19 and 21 tested in different concentrations 104

Figure S175. Effect of compounds 6, 10, 14, 16, 17, 18, 19 and 21 tested in different concentrations 105

Figure S176. Effect of compounds 6, 10, 14, 16, 17, 18, 19 and 21 tested in different concentrations 106 
Figure S1. Chromatographic trace at $254 \mathrm{~nm}$ from HPLC-PDA-HRMS-SPE-NMR analysis. Green marks indicate successful trapping of peaks 1, 2, 4, 6-10, 14, 15, and 17-21. Compounds were eluted with $\mathrm{CDCl} 3$.

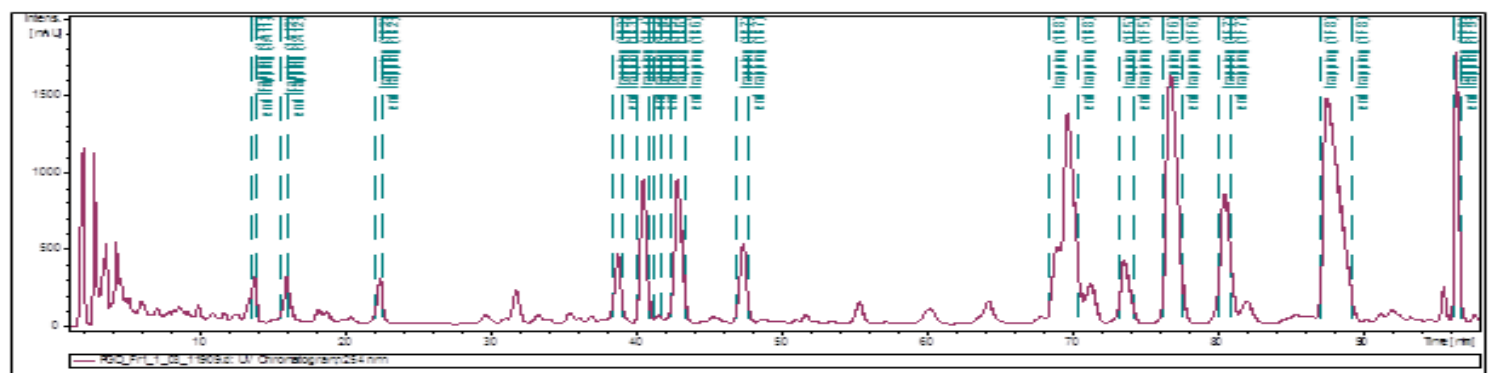

Figure S2. ${ }^{1} \mathrm{H}$ NMR spectra obtained in the HPLC-PDA-HRMS-SPE-NMR mode. Spectra of the material eluted with peaks 19 (orange), 18 (yellow), 17 (purple), 15 and 14 (green), 10 (red) and 7 (blue) in Figure S3. Compounds were eluted with CDCl3.

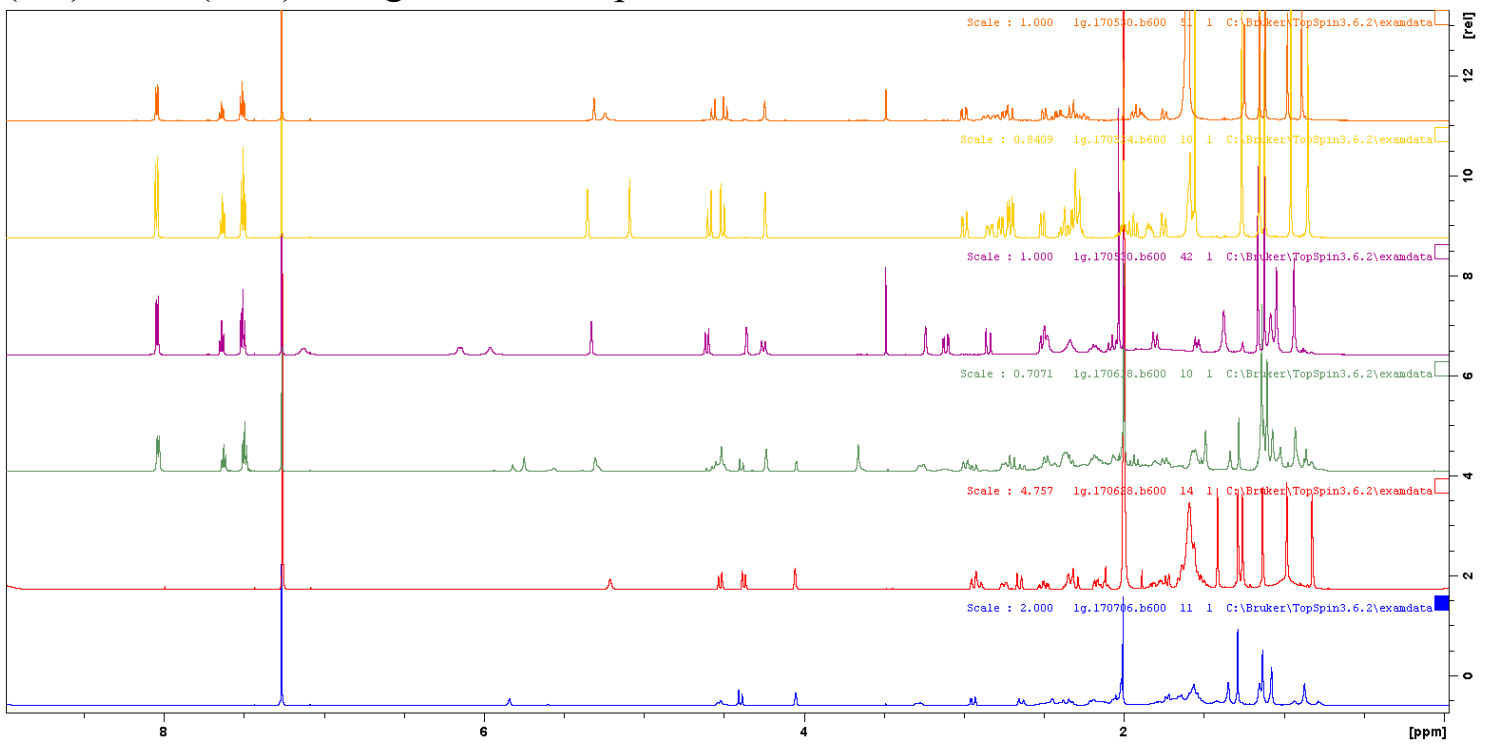


Figure S3. Direct correlation of trapped peaks 1, 2, 4, 6-10, 14, 15 and 17-21 as well as additional peaks 3, 5, 11-13, and 16 in the semipreparative HPLC chromatogram at $254 \mathrm{~nm}$. 30 repeated semipreparative separations afforded material for $2 \mathrm{D}$ NMR, ECD and in vitro assays.

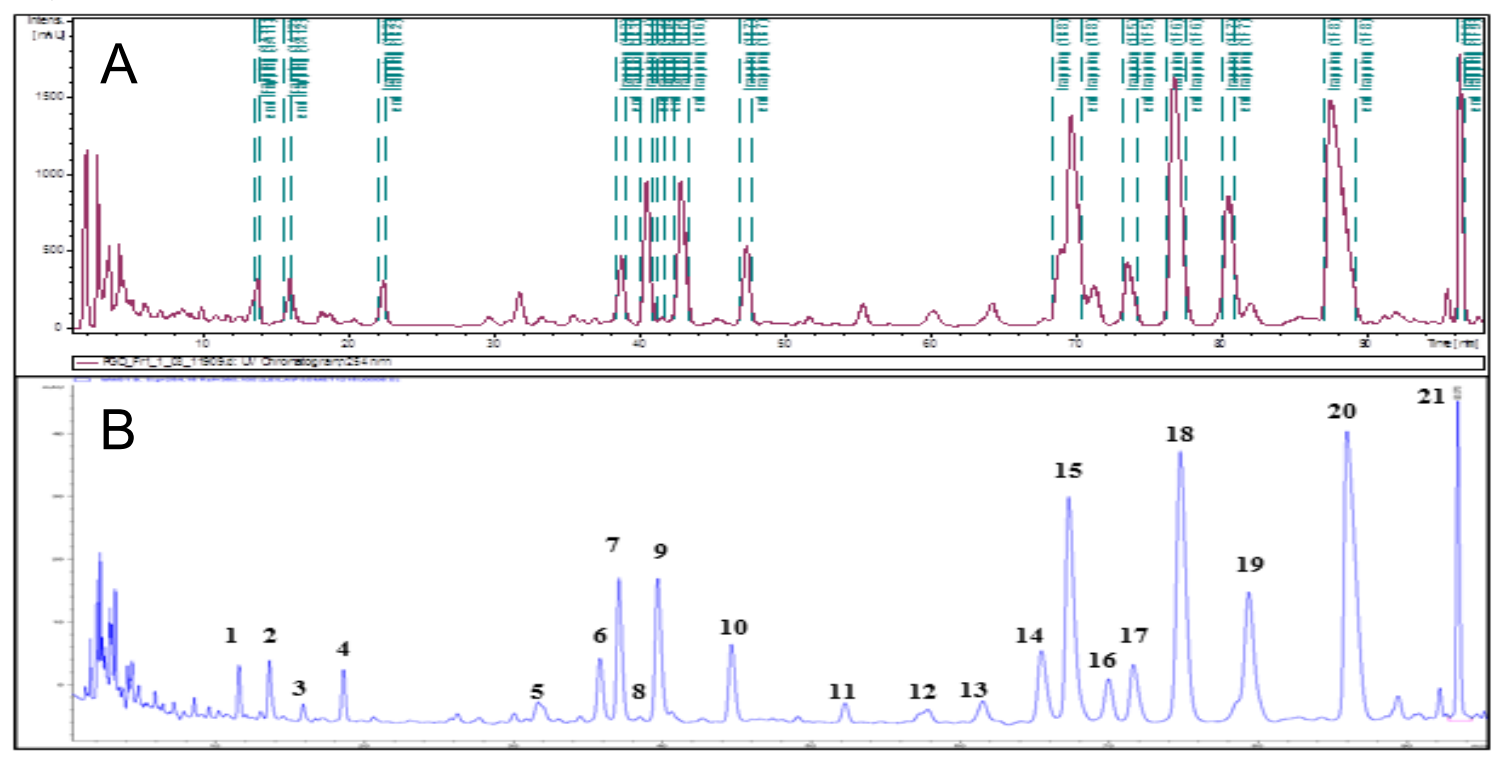


Figure S4. HRESIMS spectrum of picraviane C (1) indicating $m / z[\mathrm{M}+\mathrm{H}]^{+}=561.3051$ (calc. for $\mathrm{C}_{31} \mathrm{H}_{45} \mathrm{O}_{9}{ }^{+} 561.3058, \Delta \mathrm{M}+1.3 \mathrm{ppm}$ ), $\mathrm{m} / z\left[\mathrm{M}+\mathrm{H}-\mathrm{CH}_{3} \mathrm{CO}_{2} \mathrm{H}\right]^{+}=501.2842$ (calc. for $\mathrm{C}_{29} \mathrm{H}_{41} \mathrm{O}_{7}{ }^{+}$501.2846, $\Delta \mathrm{M}+1.0 \mathrm{ppm}$ ), $\mathrm{m} / z\left[\mathrm{M}+\mathrm{H}-\mathrm{CH}_{3} \mathrm{CO}_{2} \mathrm{H}-\mathrm{H}_{2} \mathrm{O}\right]^{+}=483.2725$ (calc. for $\mathrm{C}_{29} \mathrm{H}_{39} \mathrm{O}_{6}{ }^{+} 483.2741, \Delta \mathrm{M}+3.3 \mathrm{ppm}$ ), $m / z\left[\mathrm{M}+\mathrm{H}-\mathrm{CH}_{3} \mathrm{CO}_{2} \mathrm{H}-\left(2 \mathrm{xH}_{2} \mathrm{O}\right)\right]^{+}=465.2618$ (calc. for $\left.\mathrm{C}_{29} \mathrm{H}_{37} \mathrm{O}_{5}{ }^{+} 465.2635, \Delta \mathrm{M}+3.8 \mathrm{ppm}\right)$.

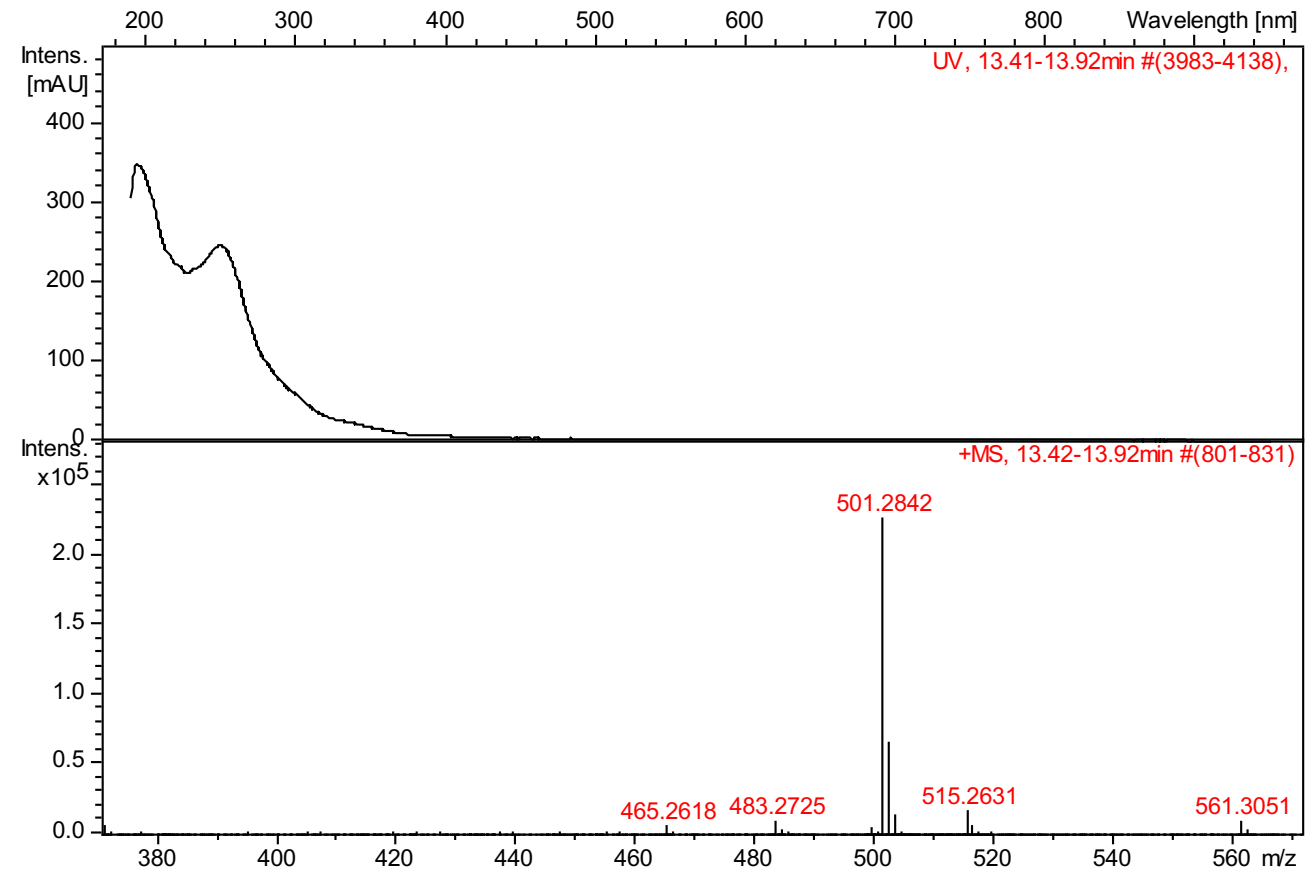

Figure S5. Mass fragmentation mechanism proposed for compounds 2 and $\mathbf{3}$.

The following mass fragmentation mechanism is proposed to explain the loss of two water molecules for compounds $\mathbf{2}$ and $\mathbf{3}$, leading to the formation of the acylium ion.

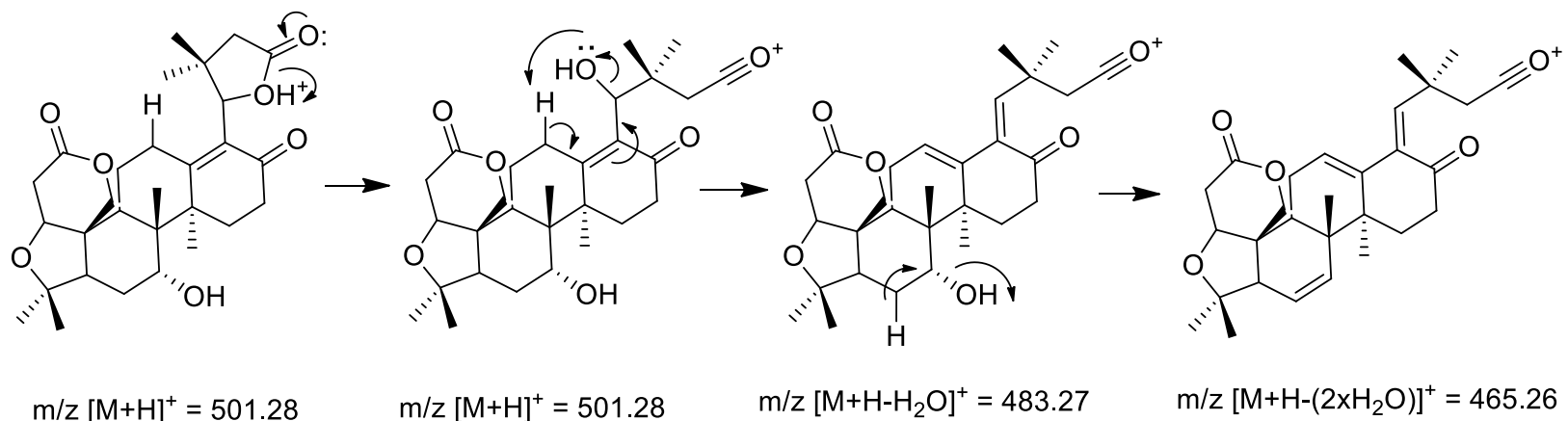


Figure S6. HRESIMS spectrum of picraviane D (2) indicating $m / z[\mathrm{M}+\mathrm{H}]^{+}=501.2829$ (calc. for $\mathrm{C}_{29} \mathrm{H}_{41} \mathrm{O}_{7}{ }^{+} 501.2846, \Delta \mathrm{M}+3.6 \mathrm{ppm}$ ), $\mathrm{m} / \mathrm{z}\left[\mathrm{M}+\mathrm{H}-\mathrm{H}_{2} \mathrm{O}\right]^{+}=483.2718$ (calc. for $\mathrm{C}_{29} \mathrm{H}_{39} \mathrm{O}_{6}{ }^{+}$ $483.2741, \Delta \mathrm{M}+4.8 \mathrm{ppm}$ ) and $\mathrm{m} / \mathrm{z}\left[\mathrm{M}+\mathrm{H}-\left(2 \mathrm{xH}_{2} \mathrm{O}\right)\right]^{+}=465.2616$ (calc. for $\mathrm{C}_{29} \mathrm{H}_{37} \mathrm{O}_{5}{ }^{+} 465.2635$, $\Delta \mathrm{M}+4.2 \mathrm{ppm})$.

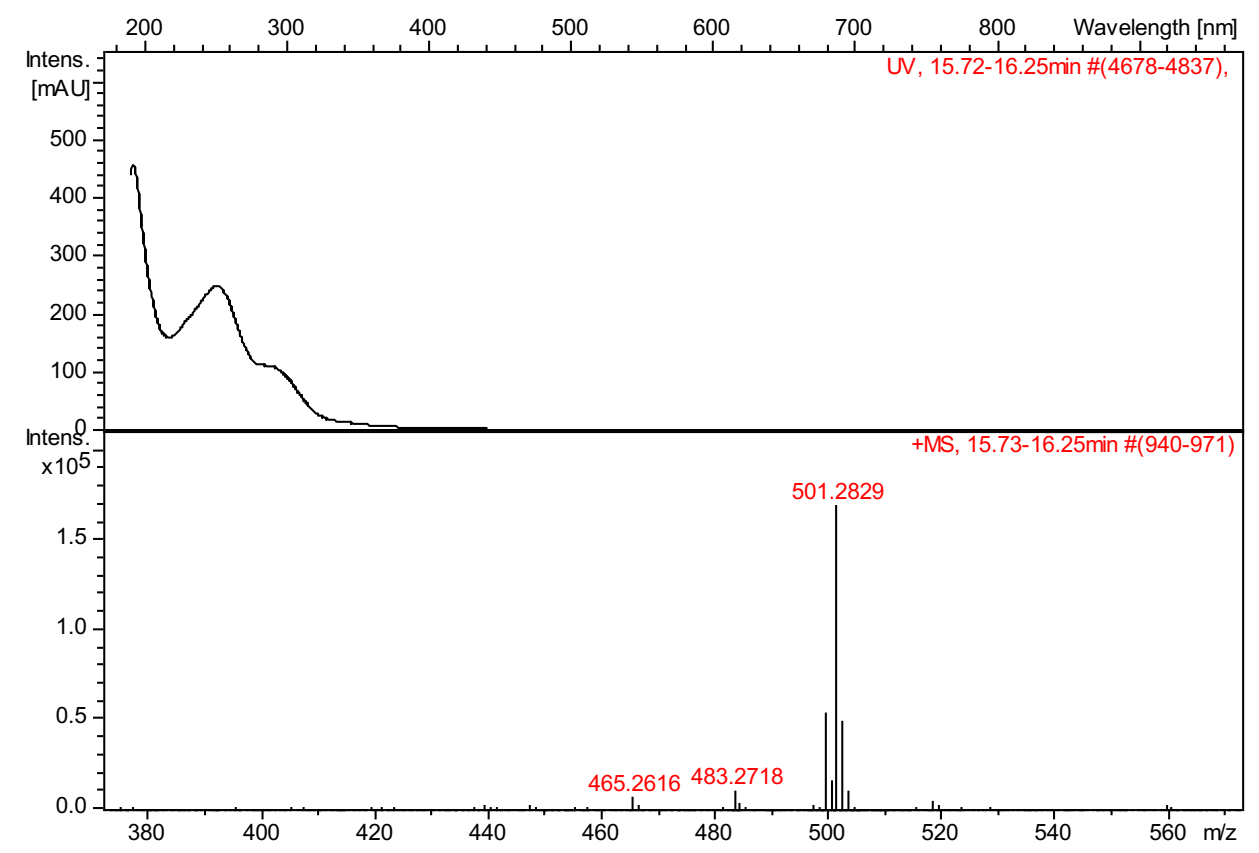

Figure S7. HRESIMS spectrum of picraviane E (3) indicating $m / z[\mathrm{M}+\mathrm{H}]^{+}=501.2842$ (calc. for $\mathrm{C}_{29} \mathrm{H}_{41} \mathrm{O}_{7}{ }^{+} 501.2846, \Delta \mathrm{M}+1.0 \mathrm{ppm}$ ), $\mathrm{m} / z\left[\mathrm{M}+\mathrm{H}-\mathrm{H}_{2} \mathrm{O}\right]^{+}=483.2730$ (calc. for $\mathrm{C}_{29} \mathrm{H}_{39} \mathrm{O}_{6}{ }^{+}$ $483.2741, \Delta \mathrm{M}+2.1 \mathrm{ppm}$ ) and $\mathrm{m} / \mathrm{z}\left[\mathrm{M}+\mathrm{H}-\left(2 \mathrm{xH}_{2} \mathrm{O}\right)\right]^{+}=465.2621$ (calc. for $\mathrm{C}_{29} \mathrm{H}_{37} \mathrm{O}_{5}{ }^{+} 465.2635$, $\Delta \mathrm{M}+3.1 \mathrm{ppm})$.

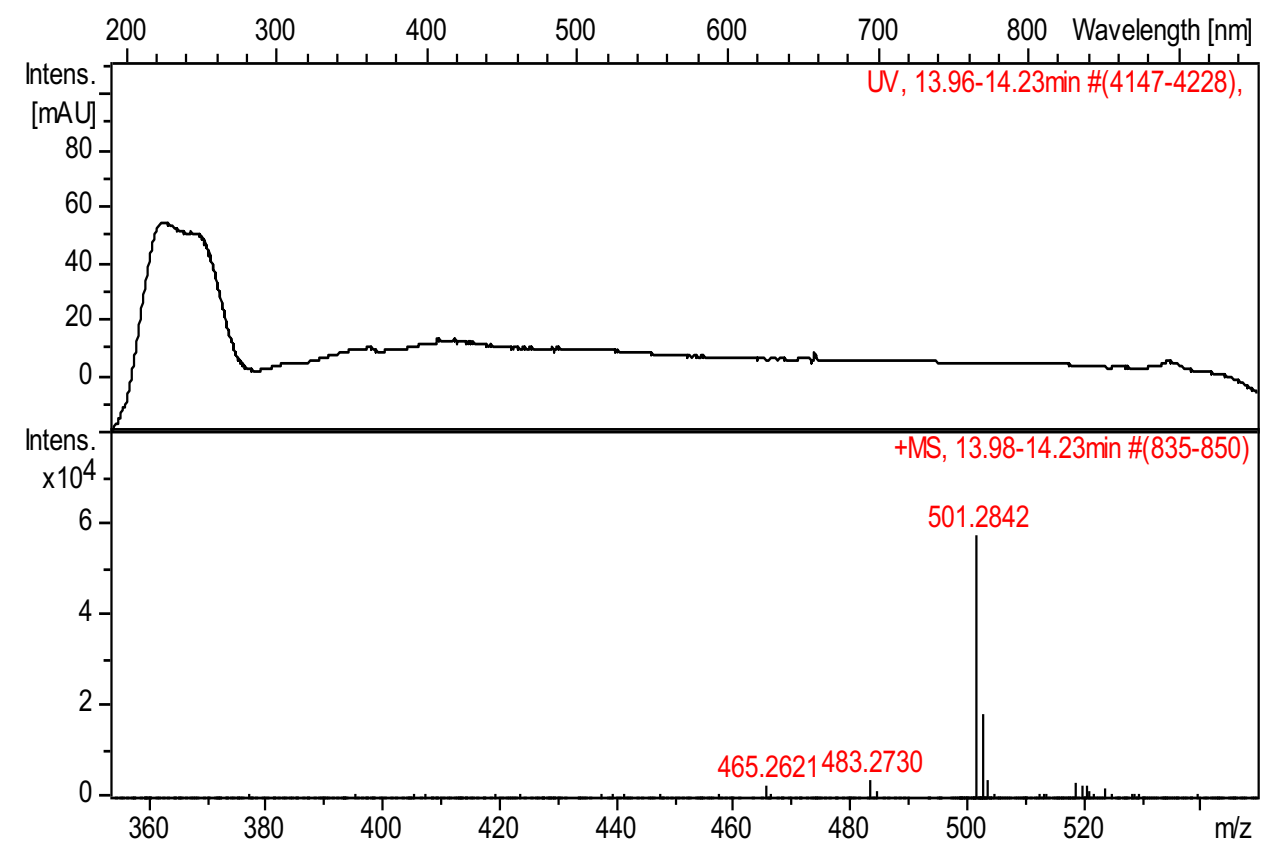


Figure S8. HRESIMS spectrum of picraviane $\mathrm{F}(5)$ indicating $\mathrm{m} / \mathrm{z}[\mathrm{M}+\mathrm{K}]^{+}=539.2411$ (calc. for $\mathrm{C}_{29} \mathrm{H}_{40} \mathrm{O}_{7} \mathrm{~K}^{+} 539.2405, \Delta \mathrm{M}-1.0 \mathrm{ppm}$ ), $\mathrm{m} / \mathrm{z}[\mathrm{M}+\mathrm{Na}]^{+}=523.2658$ (calc. for $\mathrm{C}_{29} \mathrm{H}_{40} \mathrm{O}_{7} \mathrm{Na}^{+}$ 523.2666, $\Delta \mathrm{M}+1.6 \mathrm{ppm}$ ), $\mathrm{m} / \mathrm{z}[\mathrm{M}+\mathrm{H}]^{+}=501.2828$ (calc. for $\mathrm{C}_{29} \mathrm{H}_{41} \mathrm{O}_{7}{ }^{+} 501.2846, \Delta \mathrm{M}+3.8$ $\mathrm{ppm}$ ), $\mathrm{m} / \mathrm{z}\left[\mathrm{M}+\mathrm{H}-\mathrm{H}_{2} \mathrm{O}\right]^{+}=483.2736$ (calc. for $\mathrm{C}_{29} \mathrm{H}_{39} \mathrm{O}_{6}{ }^{+} 483.2741, \Delta \mathrm{M}+1.1 \mathrm{ppm}$ ), $\mathrm{m} / z[\mathrm{M}+\mathrm{H}-$ $\left.\left(2 \mathrm{xH}_{2} \mathrm{O}\right)\right]^{+}=465.2620$ (calc. for $\left.\mathrm{C}_{29} \mathrm{H}_{37} \mathrm{O}_{5}{ }^{+} 465.2635, \Delta \mathrm{M}+3.3 \mathrm{ppm}\right)$, and $m / z\left[\mathrm{M}+\mathrm{H}-\left(2 \mathrm{xH}_{2} \mathrm{O}\right)-\right.$ $\mathrm{CO}^{+}=437.2678$ (calc. for $\mathrm{C}_{28} \mathrm{H}_{37} \mathrm{O}_{4}{ }^{+} 437.2686, \Delta \mathrm{M}+1.9 \mathrm{ppm}$ ).

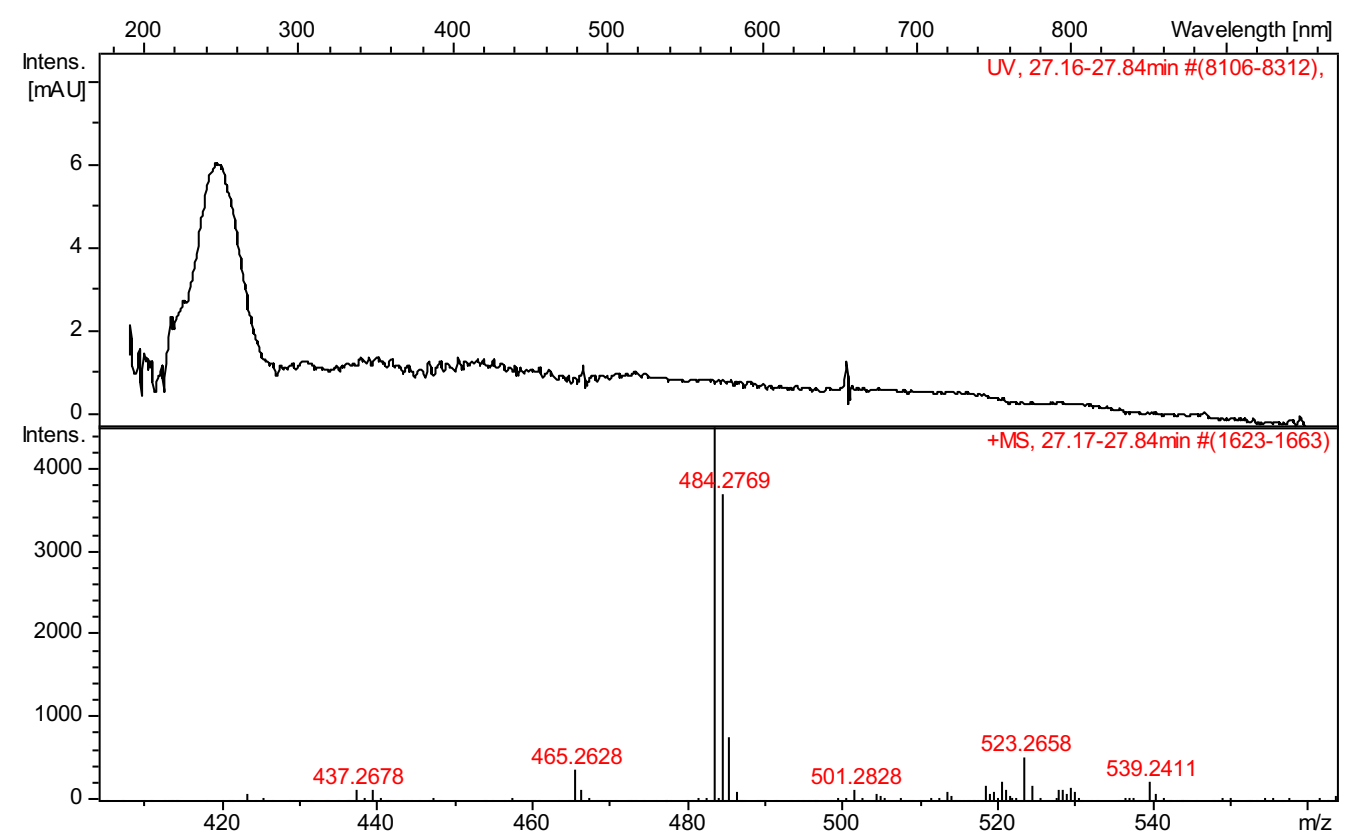

Figure S9. HRESIMS spectrum of the anthraquinone aloe-emodin (eluted together with compound 5) indicating $\mathrm{m} / \mathrm{z}[\mathrm{M}+\mathrm{H}]^{+}=271.0602$ (calc. for $\mathrm{C}_{15} \mathrm{H}_{11} \mathrm{O}_{5}{ }^{+} 271.0601, \Delta \mathrm{M}-0.4 \mathrm{ppm}$ ), $\mathrm{m} / \mathrm{z}\left[\mathrm{M}+\mathrm{H}-\mathrm{H}_{2} \mathrm{O}\right]^{+}=253.0490$ (calc. for $\mathrm{C}_{15} \mathrm{H}_{9} \mathrm{O}_{4}{ }^{+} 253.0595, \Delta \mathrm{M}+2.1 \mathrm{ppm}$ ) and $\mathrm{m} / \mathrm{z}[\mathrm{M}+\mathrm{H}-$ $\left.\mathrm{H}_{2} \mathrm{O}-\mathrm{CO}\right]^{+}=225.0546$ (calc. for $\mathrm{C}_{14} \mathrm{H}_{9} \mathrm{O}_{3}{ }^{+} 225.0546, \Delta \mathrm{M}+0.1 \mathrm{ppm}$ ).

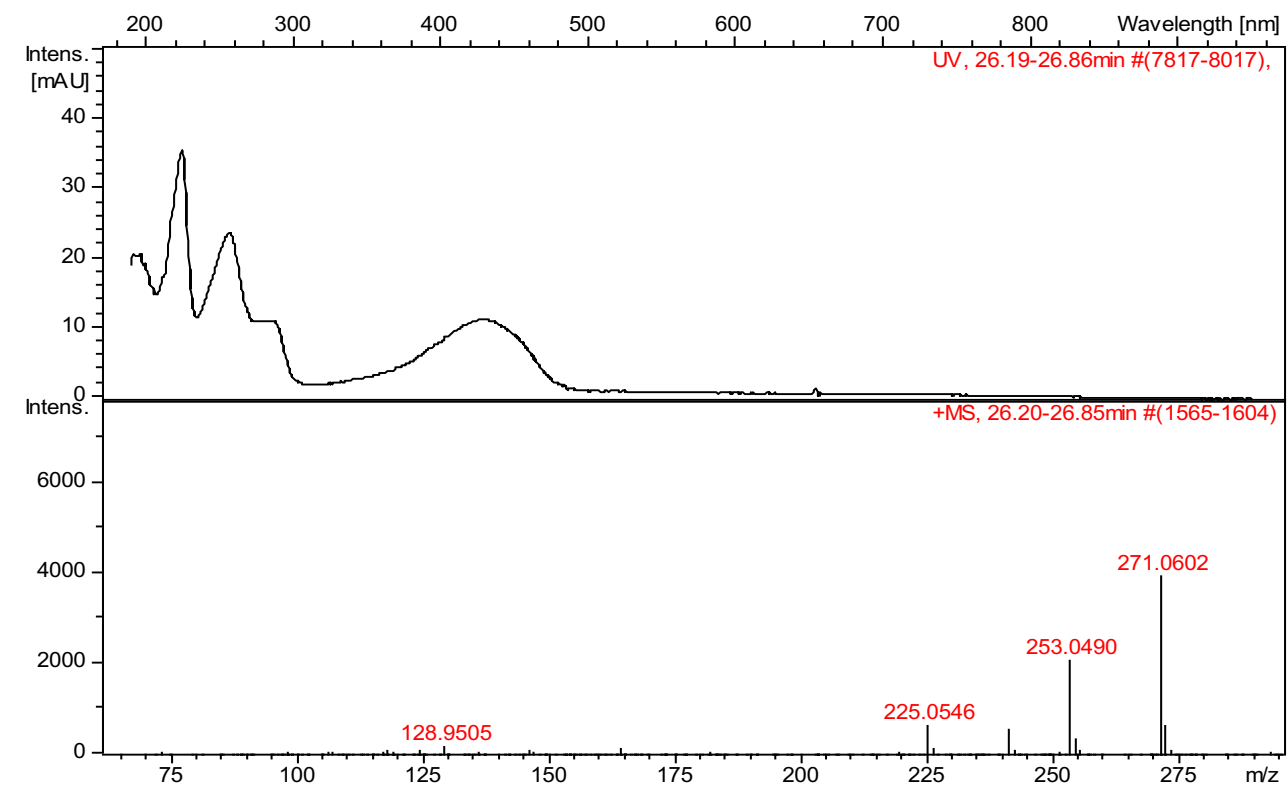


Figure S10. HRESIMS spectrum of picraviane G (6) (two different $m / z$-expansions) indicating $m / z[\mathrm{M}+\mathrm{H}]^{+}=543.2917$ (calc. for $\mathrm{C}_{31} \mathrm{H}_{43} \mathrm{O}_{8}{ }^{+} 543.2952, \Delta \mathrm{M}+6.5 \mathrm{ppm}$ ), $m / z\left[\mathrm{M}+\mathrm{H}_{-}-\mathrm{CH}_{3} \mathrm{CO}_{2} \mathrm{H}\right]^{+}$ $=483.2726$ (calc. for $\mathrm{C}_{29} \mathrm{H}_{39} \mathrm{O}_{6}{ }^{+} 483.2741, \Delta \mathrm{M}+3.1 \mathrm{ppm}$ ), $\mathrm{m} / z\left[\mathrm{M}+\mathrm{H}-\mathrm{CH}_{3} \mathrm{CO}_{2} \mathrm{H}-\mathrm{H}_{2} \mathrm{O}\right]^{+}=$ 465.2617 (calc. for $\mathrm{C}_{29} \mathrm{H}_{37} \mathrm{O}_{5}{ }^{+} 465.2635, \Delta \mathrm{M}+4.0 \mathrm{ppm}$ ).

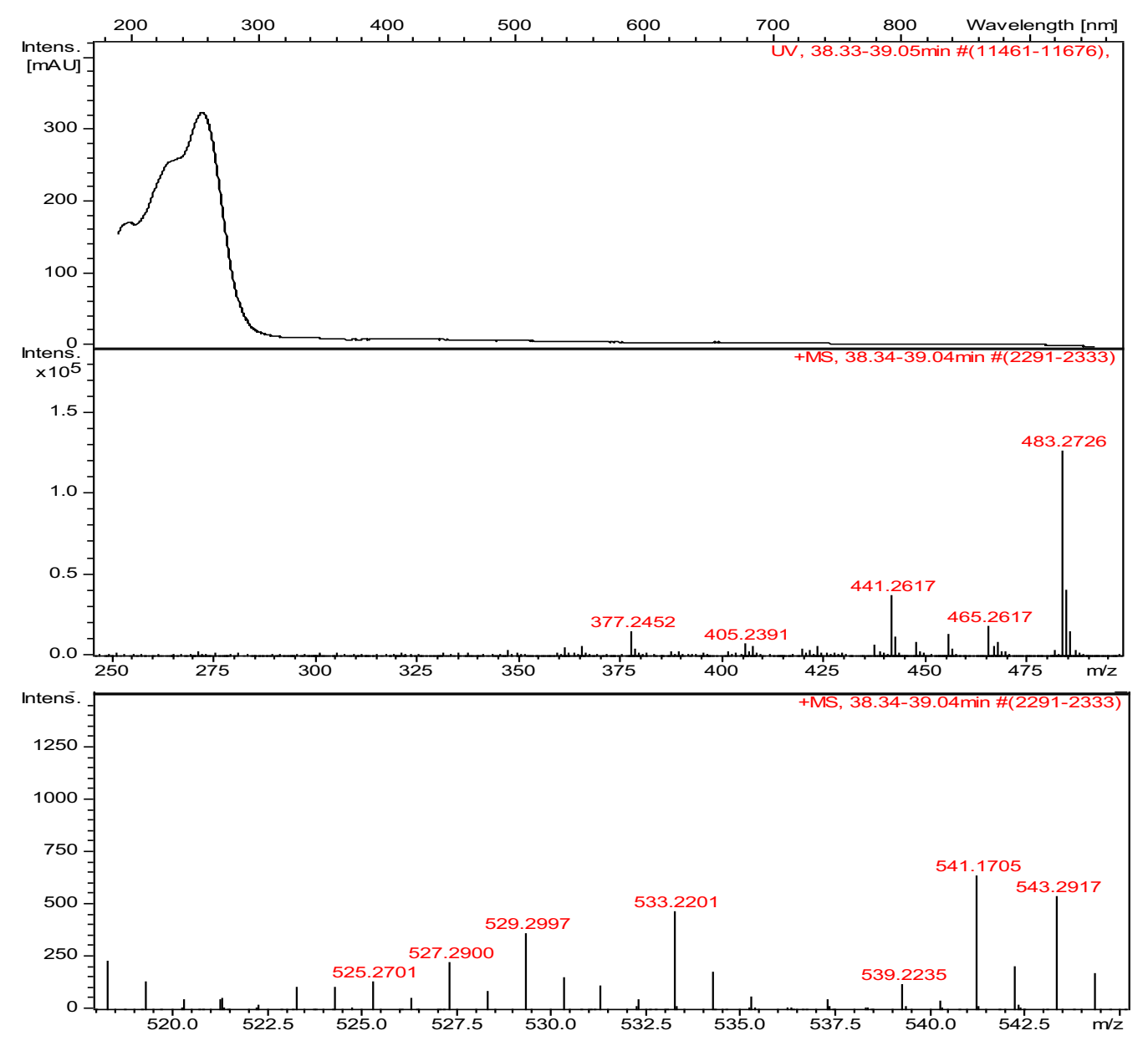


Figure S11. HRESIMS spectrum of picraviane H (7) indicating $m / z[\mathrm{M}+\mathrm{H}]^{+}=545.3096$ (calc. for $\mathrm{C}_{31} \mathrm{H}_{45} \mathrm{O}_{8}{ }^{+} 545.3108, \Delta \mathrm{M}+2.4 \mathrm{ppm}$ ), $\mathrm{m} / z\left[\mathrm{M}+\mathrm{H}-\mathrm{CH}_{3} \mathrm{CO}_{2} \mathrm{H}\right]^{+}=485.2893$ (calc. for $\mathrm{C}_{29} \mathrm{H}_{41} \mathrm{O}_{6}{ }^{+} 485.2897, \Delta \mathrm{M}+1.0 \mathrm{ppm}$ ), $\mathrm{m} / z\left[\mathrm{M}+\mathrm{H}-\mathrm{CH}_{3} \mathrm{CO}_{2} \mathrm{H}-\mathrm{H}_{2} \mathrm{O}\right]^{+}=467.2777$ (calc. for $\left.\mathrm{C}_{29} \mathrm{H}_{39} \mathrm{O}_{5}{ }^{+} 467.2792, \Delta \mathrm{M}+3.2 \mathrm{ppm}\right)$.

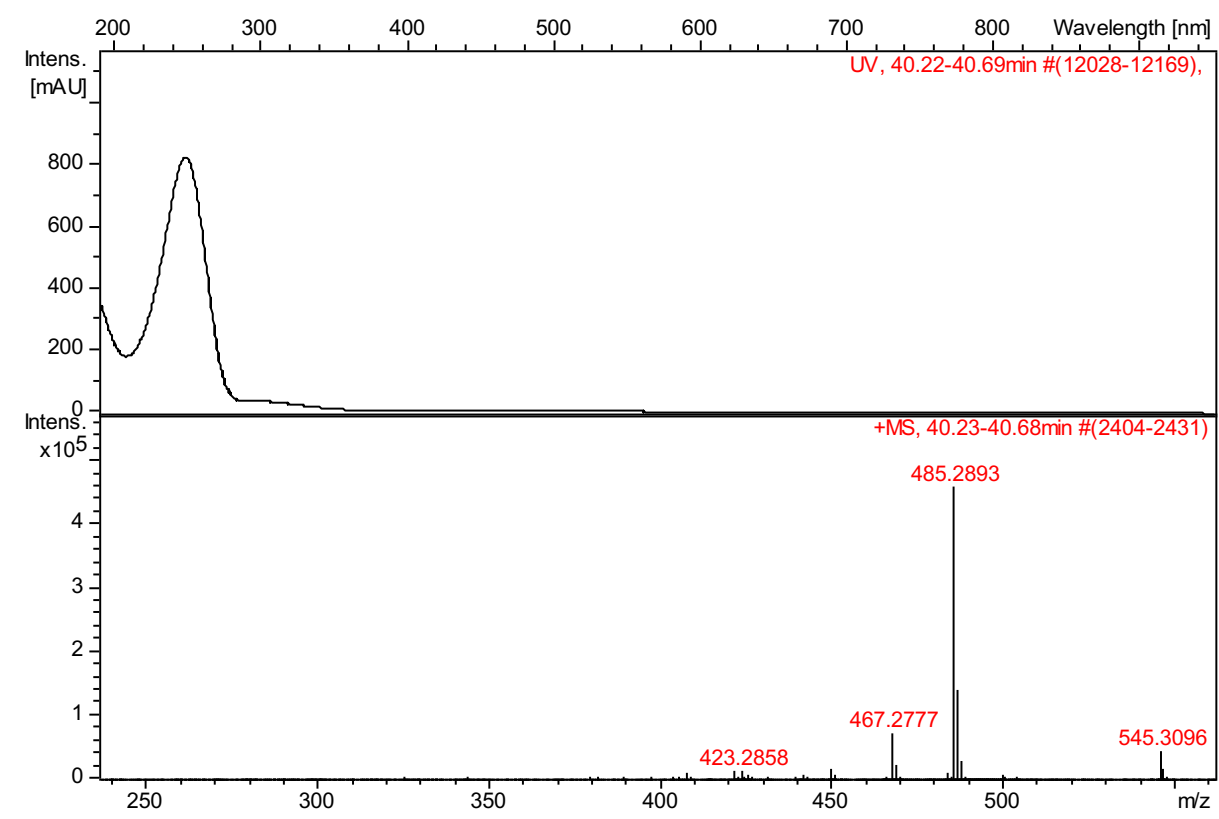


Figure S12. HRESIMS spectrum of picraviane I (8) (two different $m / z$-expansions) indicating $m / z[\mathrm{M}+\mathrm{H}]^{+}=543.2937$ (calc. for $\mathrm{C}_{31} \mathrm{H}_{43} \mathrm{O}_{8}{ }^{+} 543.2952, \Delta \mathrm{M}+2.8 \mathrm{ppm}$ ), $m / z\left[\mathrm{M}+\mathrm{H}_{-}-\mathrm{CH}_{3} \mathrm{CO}_{2} \mathrm{H}\right]^{+}$ $=483.2734$ (calc. for $\mathrm{C}_{29} \mathrm{H}_{39} \mathrm{O}_{6}{ }^{+} 483.2741, \Delta \mathrm{M}+1.5 \mathrm{ppm}$ ), $m / z\left[\mathrm{M}+\mathrm{H}-\mathrm{CH}_{3} \mathrm{CO}_{2} \mathrm{H}_{-}-\mathrm{H}_{2} \mathrm{O}\right]^{+}=$ 465.2618 (calc. for $\mathrm{C}_{29} \mathrm{H}_{37} \mathrm{O}_{5}{ }^{+} 465.2635, \Delta \mathrm{M}+3.8 \mathrm{ppm}$ ), and $m / z\left[\mathrm{M}+\mathrm{H}-\mathrm{CH}_{3} \mathrm{CO}_{2} \mathrm{H}-\mathrm{H}_{2} \mathrm{O}-\mathrm{CO}\right]^{+}$ $=437.2693$ (calc. for $\mathrm{C}_{28} \mathrm{H}_{37} \mathrm{O}_{4}{ }^{+} 437.2686, \Delta \mathrm{M}-1.5 \mathrm{ppm}$ ).
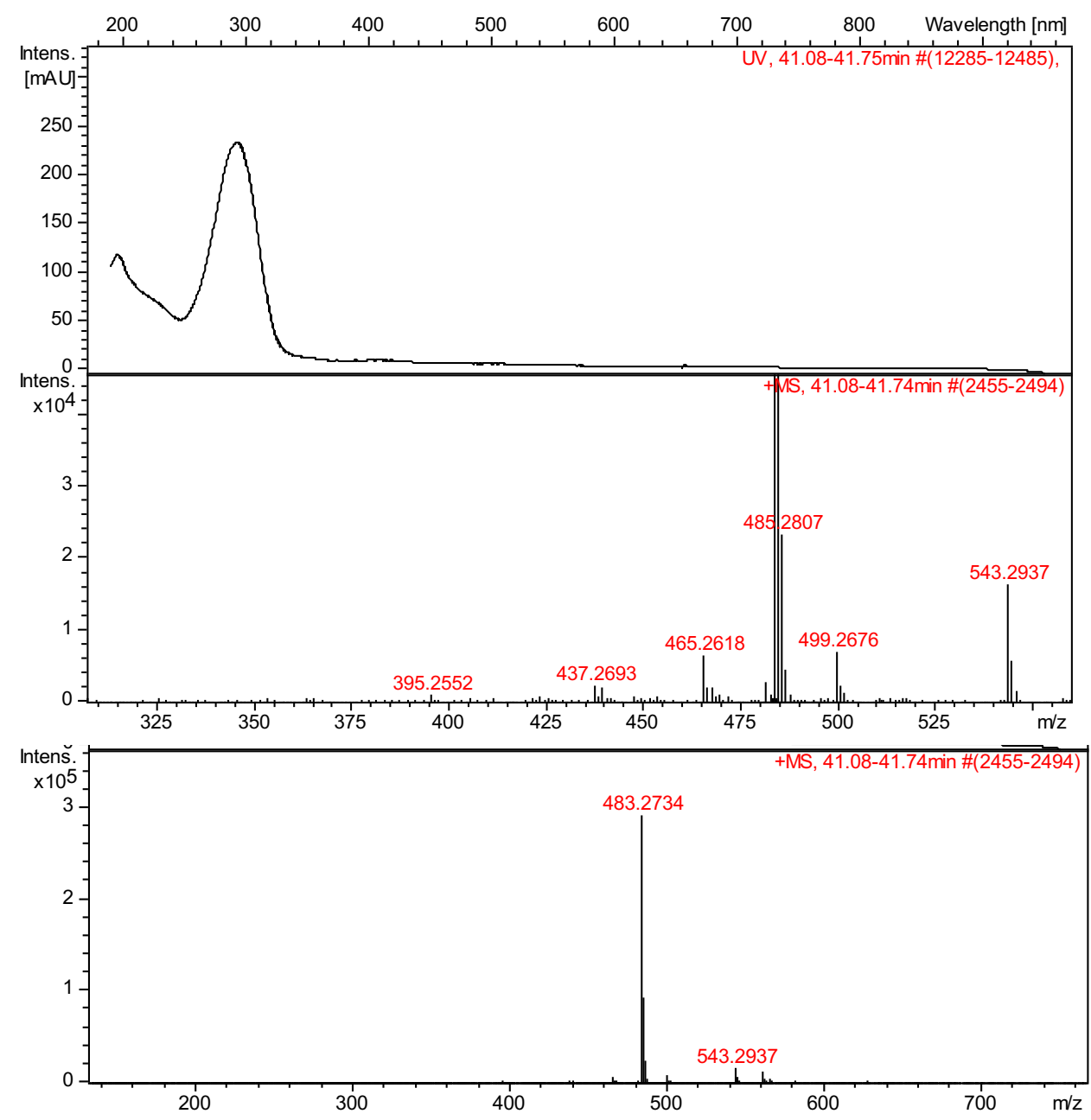
Figure S13. HRESIMS spectrum of picraviane J (9) indicating $m / z[\mathrm{M}+\mathrm{H}]^{+}=485.2890$ (calc. for $\mathrm{C}_{29} \mathrm{H}_{41} \mathrm{O}_{6}{ }^{+}$485.2898, $\Delta \mathrm{M}+1.6 \mathrm{ppm}$ ).

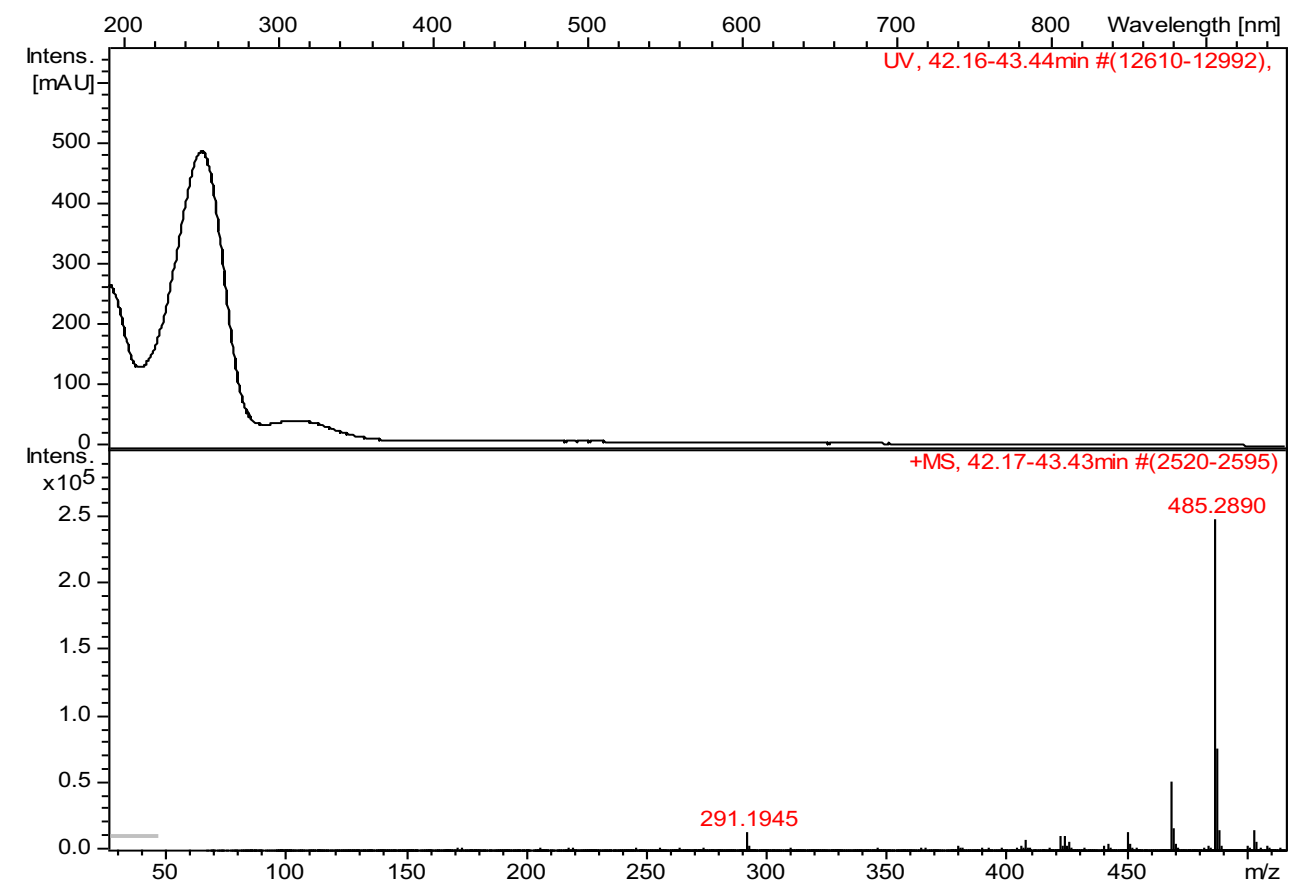

Figure S14. HRESIMS spectrum of picraviane K (10) indicating $m / z[\mathrm{M}+\mathrm{H}]^{+}=485.2886$ (calc. for $\mathrm{C}_{29} \mathrm{H}_{41} \mathrm{O}_{6}{ }^{+} 485.2898, \Delta \mathrm{M}+2.4 \mathrm{ppm}$ ).

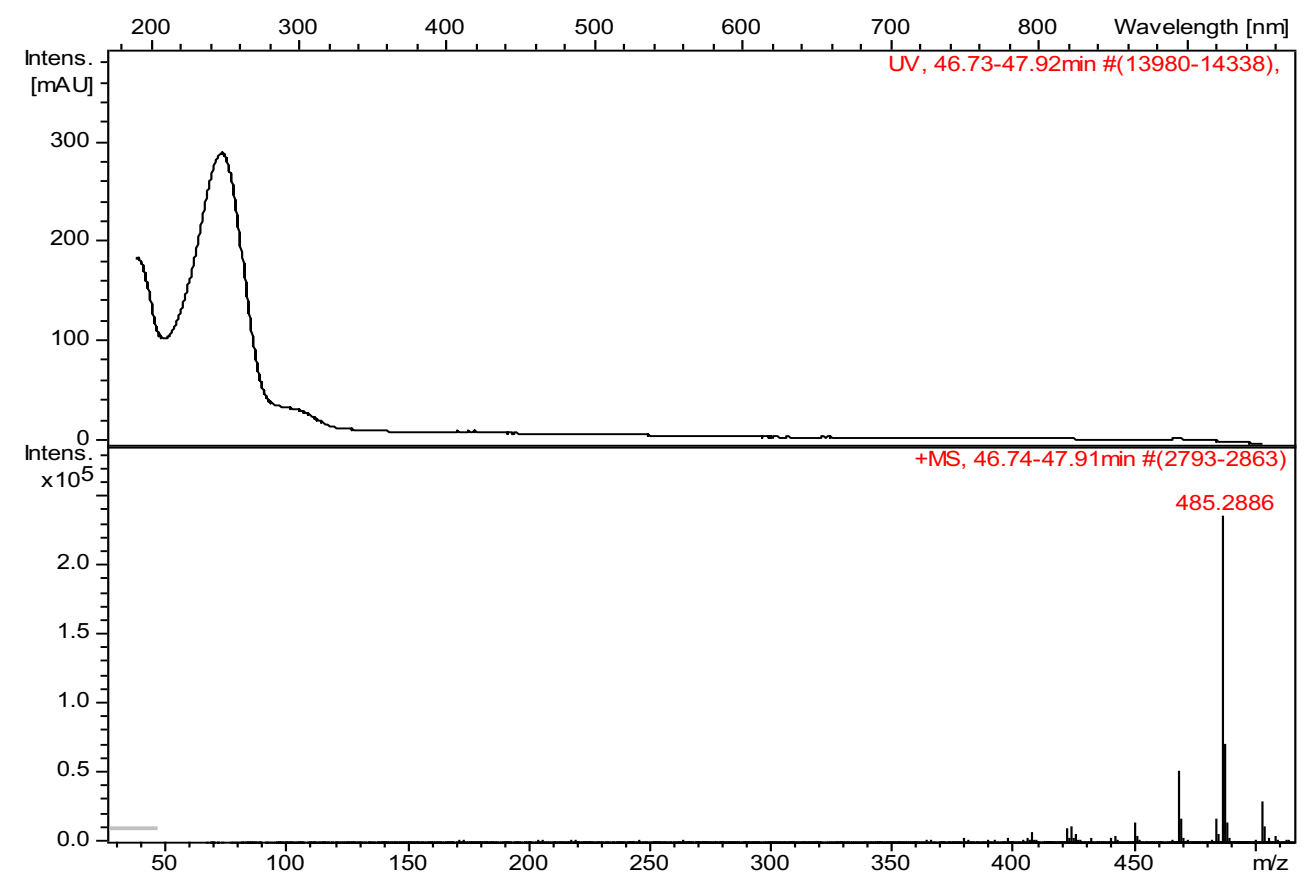


Figure S15. HRESIMS spectrum of picraviane L (12) indicating $m / z[\mathrm{M}+\mathrm{H}]^{+}=621.3051$ (calc. for $\mathrm{C}_{36} \mathrm{H}_{45} \mathrm{O}_{9}{ }^{+} 621.3058, \Delta \mathrm{M}+1.1 \mathrm{ppm}$ ), $m / z\left[\mathrm{M}+\mathrm{H}_{-} \mathrm{H}_{2} \mathrm{O}\right]^{+}=603.2953$ (calc. for $\mathrm{C}_{36} \mathrm{H}_{43} \mathrm{O}_{8}{ }^{+}$ 603.2952, $\Delta \mathrm{M}-0.1 \mathrm{ppm}$ ), $m / z\left[\mathrm{M}+\mathrm{H}-\left(2 \mathrm{xH}_{2} \mathrm{O}\right)\right]^{+}=585.2842$ (calc. for $\mathrm{C}_{36} \mathrm{H}_{41} \mathrm{O}_{7}+585.2846, \Delta \mathrm{M}$ $+0.8 \mathrm{ppm}$ ), $m / z\left[\mathrm{M}+\mathrm{H}-\mathrm{H}_{2} \mathrm{O}-\mathrm{C}_{6} \mathrm{H}_{5} \mathrm{CO}_{2} \mathrm{H}\right]^{+}=481.2578$ (calc. for $\mathrm{C}_{29} \mathrm{H}_{37} \mathrm{O}_{6}{ }^{+} 481.2584, \Delta \mathrm{M}+1.4$ ppm).

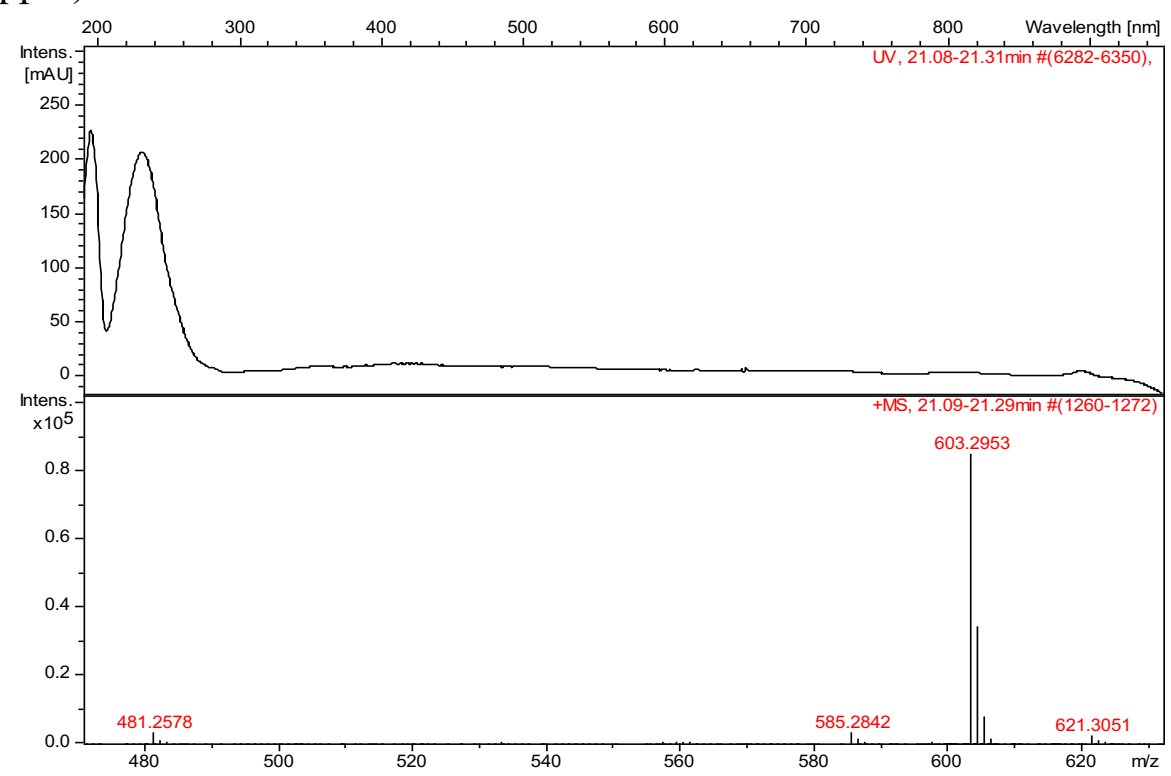

Figure S16. HRESIMS spectrum of picraviane M (13) indicating $m / z[\mathrm{M}+\mathrm{H}]^{+}=621.3046$ (calc. for $\mathrm{C}_{36} \mathrm{H}_{45} \mathrm{O}_{9}{ }^{+} 621.3058, \Delta \mathrm{M}+1.9 \mathrm{ppm}$ ), $\mathrm{m} / z\left[\mathrm{M}+\mathrm{H}-\mathrm{H}_{2} \mathrm{O}\right]^{+}=603.2944$ (calc. for $\mathrm{C}_{36} \mathrm{H}_{43} \mathrm{O}_{8}{ }^{+}$ 603.2952, $\Delta \mathrm{M}+1.4 \mathrm{ppm}$ ), $\mathrm{m} / z\left[\mathrm{M}+\mathrm{H}-\left(2 \mathrm{xH}_{2} \mathrm{O}\right)\right]^{+}=585.2841$ (calc. for $\mathrm{C}_{36} \mathrm{H}_{41} \mathrm{O}_{7}{ }^{+}$585.2846, $\Delta \mathrm{M}+1.0 \mathrm{ppm}$ ), $m / z\left[\mathrm{M}+\mathrm{H}-\mathrm{H}_{2} \mathrm{O}-\mathrm{C}_{6} \mathrm{H}_{5} \mathrm{CO}_{2} \mathrm{H}\right]^{+}=481.2569$ (calc. for $\mathrm{C}_{29} \mathrm{H}_{37} \mathrm{O}_{6}{ }^{+} 481.2584, \Delta \mathrm{M}$ $+3.3 \mathrm{ppm})$.

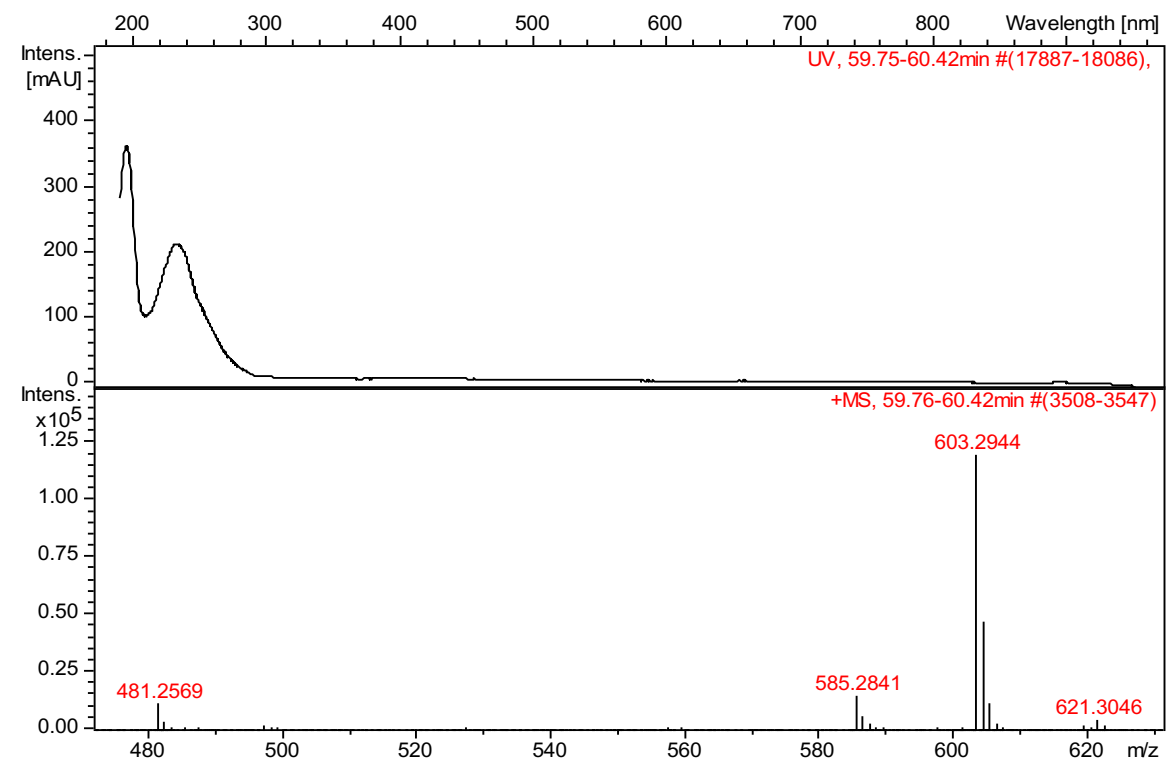


Figure S17. HRESIMS spectrum of picraviane $\mathrm{N}(14)$ indicating $m / z[\mathrm{M}+\mathrm{H}]^{+}=559.3251$ (calc. for $\mathrm{C}_{32} \mathrm{H}_{47} \mathrm{O}_{8}{ }^{+} 559.3265, \Delta \mathrm{M}+2.6 \mathrm{ppm}$ ), $\mathrm{m} / z\left[\mathrm{M}+\mathrm{H}-\mathrm{CH}_{3} \mathrm{CO}_{2} \mathrm{H}\right]^{+}=499.3054$ (calc. for $\mathrm{C}_{30} \mathrm{H}_{43} \mathrm{O}_{6}{ }^{+} 499.3054, \Delta \mathrm{M} 0.0 \mathrm{ppm}$ ) and $\mathrm{m} / z\left[\mathrm{M}+\mathrm{H}-\mathrm{CH}_{3} \mathrm{CO}_{2} \mathrm{H}-\mathrm{CH}_{3} \mathrm{OH}\right]^{+}=467.2776$ (calc. for $\mathrm{C}_{29} \mathrm{H}_{39} \mathrm{O}_{5}{ }^{+}$467.2792, $\left.\Delta \mathrm{M}+3.4 \mathrm{ppm}\right)$.

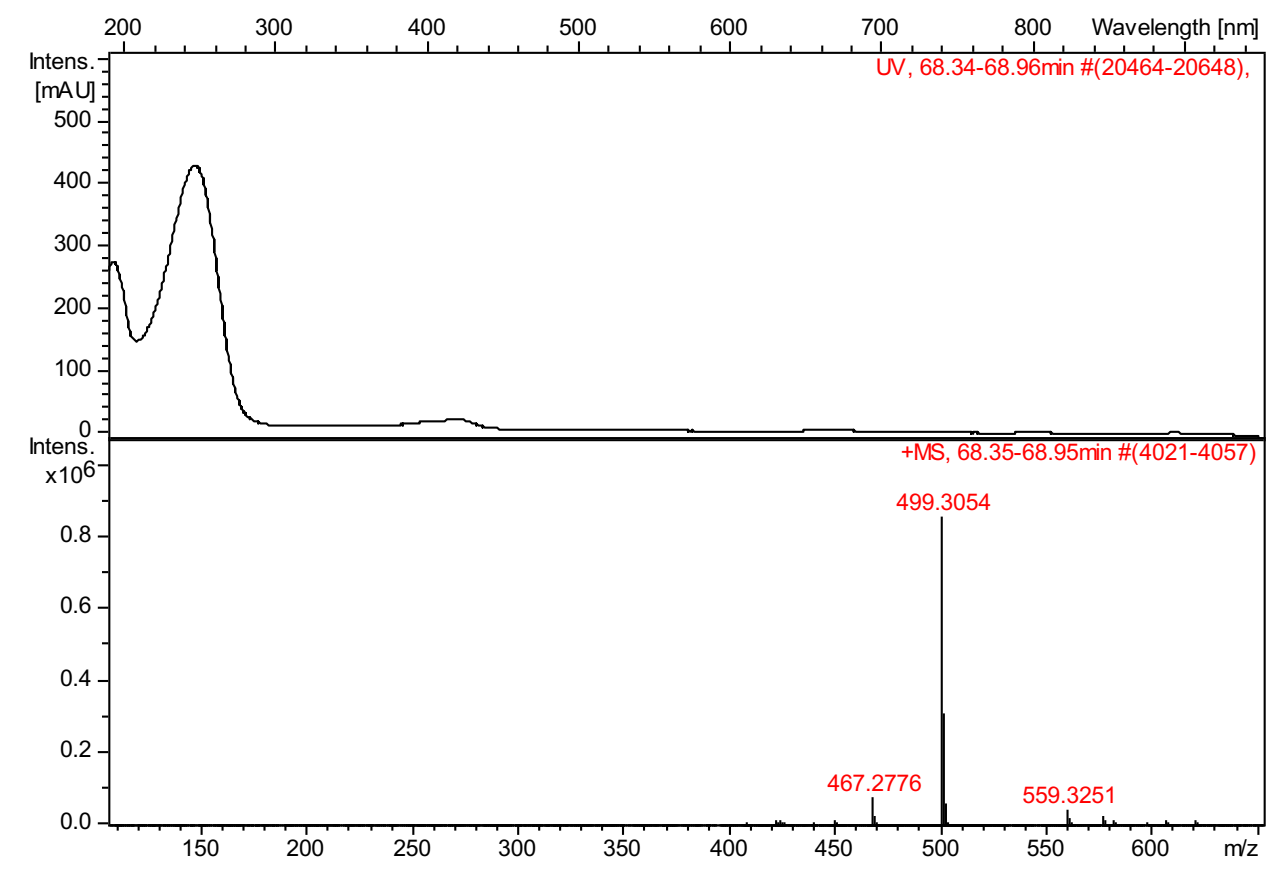


Figure S18. HRESIMS spectrum of picraviane $O$ (15) (two different $m / z$-expansions) indicating $m / z[\mathrm{M}+\mathrm{H}]^{+}=665.3325$ (calc. for $\mathrm{C}_{38} \mathrm{H}_{49} \mathrm{O}_{10}{ }^{+}$665.3320, $\Delta \mathrm{M}-0.7 \mathrm{ppm}$ ), $\mathrm{m} / z[\mathrm{M}+\mathrm{H}-$ $\left.\mathrm{CH}_{3} \mathrm{CO}_{2} \mathrm{H}\right]^{+}=605.3116$ (calc. for $\mathrm{C}_{36} \mathrm{H}_{45} \mathrm{O}_{8}{ }^{+}$605.3108, $\Delta \mathrm{M}-1.2 \mathrm{ppm}$ ), $m / z\left[\mathrm{M}+\mathrm{H}_{-} \mathrm{CH}_{3} \mathrm{CO}_{2} \mathrm{H}-\right.$ $\left.\mathrm{H}_{2} \mathrm{O}\right]^{+}=587.3000$ (calc. for $\mathrm{C}_{36} \mathrm{H}_{43} \mathrm{O}_{7}{ }^{+}$587.3003, $\Delta \mathrm{M}+0.6 \mathrm{ppm}$ ), $m / z\left[\mathrm{M}+\mathrm{H}-\mathrm{CH}_{3} \mathrm{CO}_{2} \mathrm{H}-\right.$ $\left.\mathrm{C}_{6} \mathrm{H}_{5} \mathrm{CO}_{2} \mathrm{H}\right]^{+}=483.2736$ (calc. for $\mathrm{C}_{29} \mathrm{H}_{39} \mathrm{O}_{6}{ }^{+} 483.2741, \Delta \mathrm{M}+1.1 \mathrm{ppm}$ ) and $\mathrm{m} / z[\mathrm{M}+\mathrm{H}-$ $\left.\mathrm{CH}_{3} \mathrm{CO}_{2} \mathrm{H}-\mathrm{C}_{6} \mathrm{H}_{5} \mathrm{CO}_{2} \mathrm{H}-\mathrm{H}_{2} \mathrm{O}\right]^{+}=465.2630$ (calc. for $\mathrm{C}_{29} \mathrm{H}_{37} \mathrm{O}_{5}{ }^{+} 465.2635, \Delta \mathrm{M}+1.2 \mathrm{ppm}$ ).

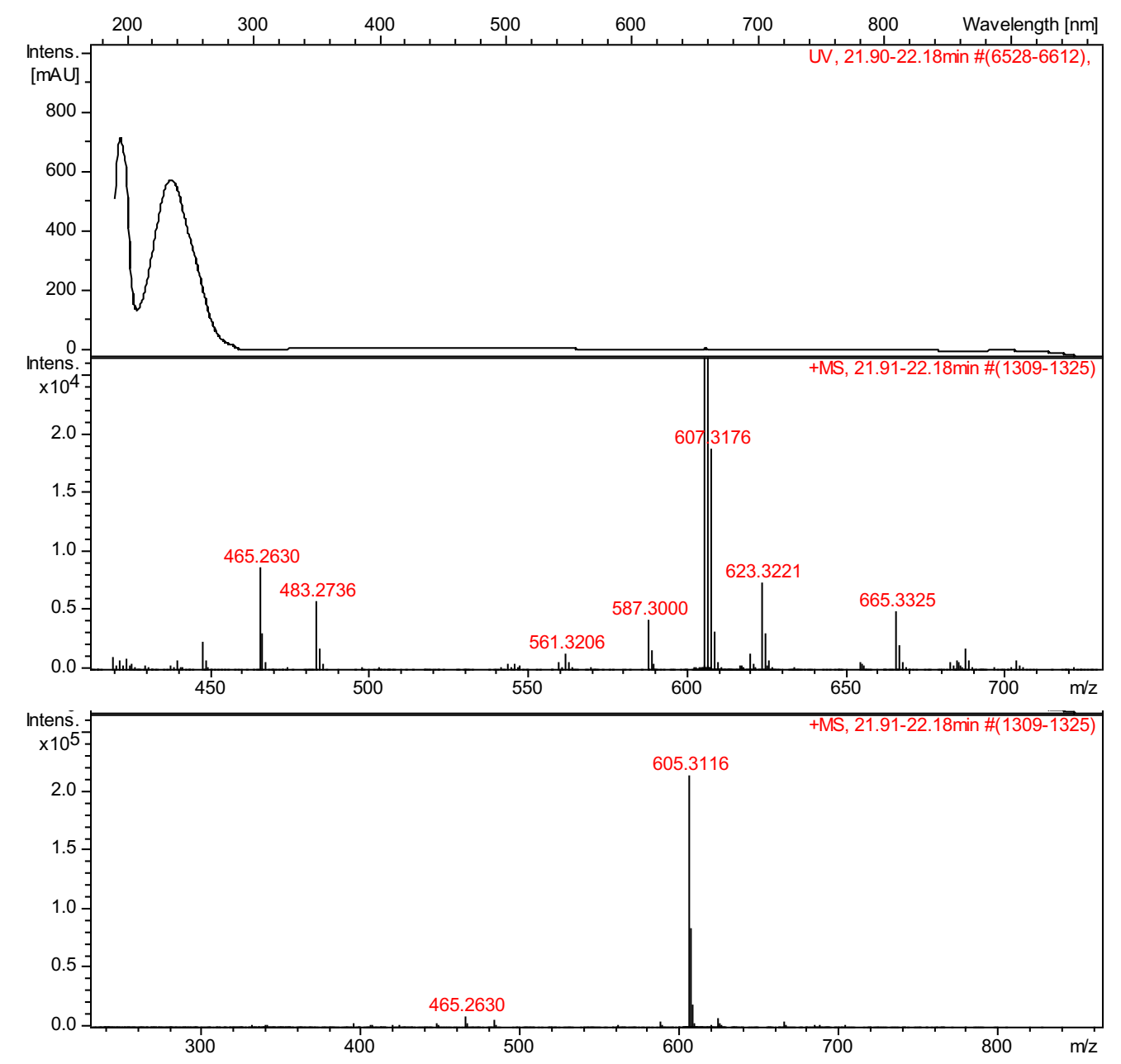


Figure S19. HRESIMS spectrum of picraviane $\mathrm{P}(\mathbf{1 6})$ (two different $m / z$-expansions) indicating $m / z[\mathrm{M}+\mathrm{H}]^{+}=679.3087$ (calc. for $\mathrm{C}_{38} \mathrm{H}_{47} \mathrm{O}_{11}{ }^{+} 679.3112, \Delta \mathrm{M}+3.8 \mathrm{ppm}$ ), $\mathrm{m} / z[\mathrm{M}+\mathrm{H}-$ $\left.\mathrm{CH}_{3} \mathrm{CO}_{2} \mathrm{H}\right]^{+}=619.2888$ (calc. for $\mathrm{C}_{36} \mathrm{H}_{43} \mathrm{O}_{9}{ }^{+} 619.2901, \Delta \mathrm{M}+2.2 \mathrm{ppm}$ ), $m / z\left[\mathrm{M}+\mathrm{H}_{-} \mathrm{CH}_{3} \mathrm{CO}_{2} \mathrm{H}-\right.$ $\left.\mathrm{H}_{2} \mathrm{O}\right]^{+}=601.2777$ (calc. for $\mathrm{C}_{36} \mathrm{H}_{41} \mathrm{O}_{8}{ }^{+} 601.2795, \Delta \mathrm{M}+3.2 \mathrm{ppm}$ ), $m / z\left[\mathrm{M}+\mathrm{H}-\mathrm{CH}_{3} \mathrm{CO}_{2} \mathrm{H}-\right.$ $\left.\mathrm{C}_{6} \mathrm{H}_{5} \mathrm{CO}_{2} \mathrm{H}\right]^{+}=497.2522$ (calc. for $\mathrm{C}_{29} \mathrm{H}_{37} \mathrm{O}_{7}^{+} 497.2533, \Delta \mathrm{M}+2.4 \mathrm{ppm}$ ) and $\mathrm{m} / z[\mathrm{M}+\mathrm{H}-$ $\left.\mathrm{CH}_{3} \mathrm{CO}_{2} \mathrm{H}-\mathrm{C}_{6} \mathrm{H}_{5} \mathrm{CO}_{2} \mathrm{H}-\mathrm{H}_{2} \mathrm{O}\right]^{+}=479.2412$, (calc. for $\mathrm{C}_{29} \mathrm{H}_{35} \mathrm{O}_{6}{ }^{+} 479.2428, \Delta \mathrm{M}+3.4 \mathrm{ppm}$ ).
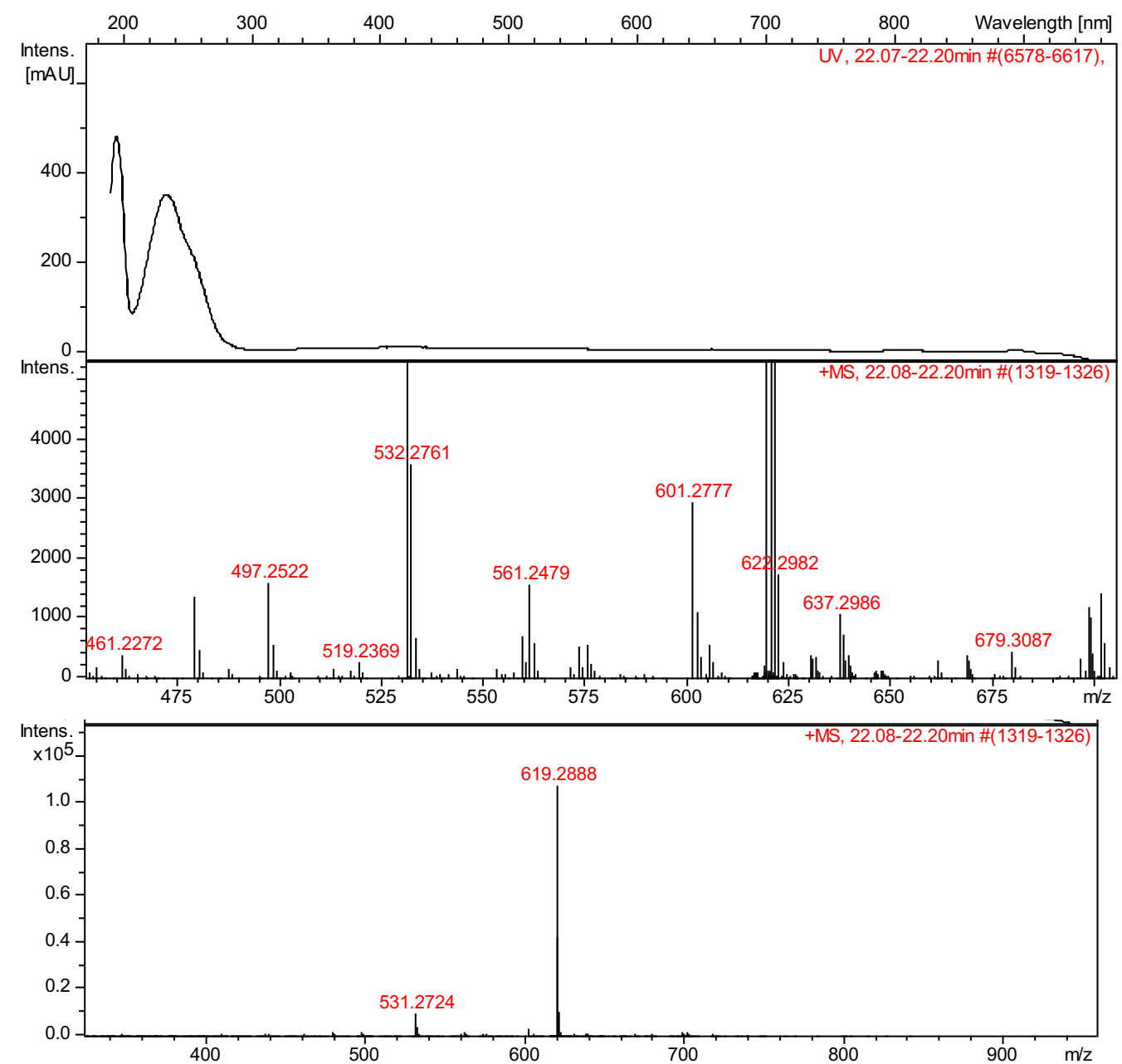
Figure S20. HRESIMS spectrum of picraviane Q (17) (two different $m / z$-expansions) indicating $m / z[\mathrm{M}+\mathrm{H}]^{+}=663.3147$ (calc. for $\mathrm{C}_{38} \mathrm{H}_{47} \mathrm{O}_{10}{ }^{+} 663.3163, \Delta \mathrm{M}+2.5 \mathrm{ppm}$ ), $m / z[\mathrm{M}+\mathrm{H}-$ $\left.\mathrm{CH}_{3} \mathrm{CO}_{2} \mathrm{H}\right]^{+}=603.2947$ (calc. for $\mathrm{C}_{36} \mathrm{H}_{43} \mathrm{O}_{8}{ }^{+}$603.2952, $\Delta \mathrm{M}+0.9 \mathrm{ppm}$ ), $m / z\left[\mathrm{M}+\mathrm{H}-\mathrm{CH}_{3} \mathrm{CO}_{2} \mathrm{H}-\right.$ $\left.\mathrm{H}_{2} \mathrm{O}\right]^{+}=585.2835$ (calc. for $\mathrm{C}_{36} \mathrm{H}_{41} \mathrm{O}_{7}{ }^{+} 585.2846, \Delta \mathrm{M}+2.0 \mathrm{ppm}$ ) and $m / z\left[\mathrm{M}+\mathrm{H}-\mathrm{CH}_{3} \mathrm{CO}_{2} \mathrm{H}-\right.$ $\left.\mathrm{C}_{6} \mathrm{H}_{5} \mathrm{CO}_{2} \mathrm{H}-\mathrm{H}_{2} \mathrm{O}\right]^{+}=463.2468$ (calc. for $\mathrm{C}_{29} \mathrm{H}_{35} \mathrm{O}_{5}{ }^{+} 463.2479, \Delta \mathrm{M}+2.4 \mathrm{ppm}$ ).

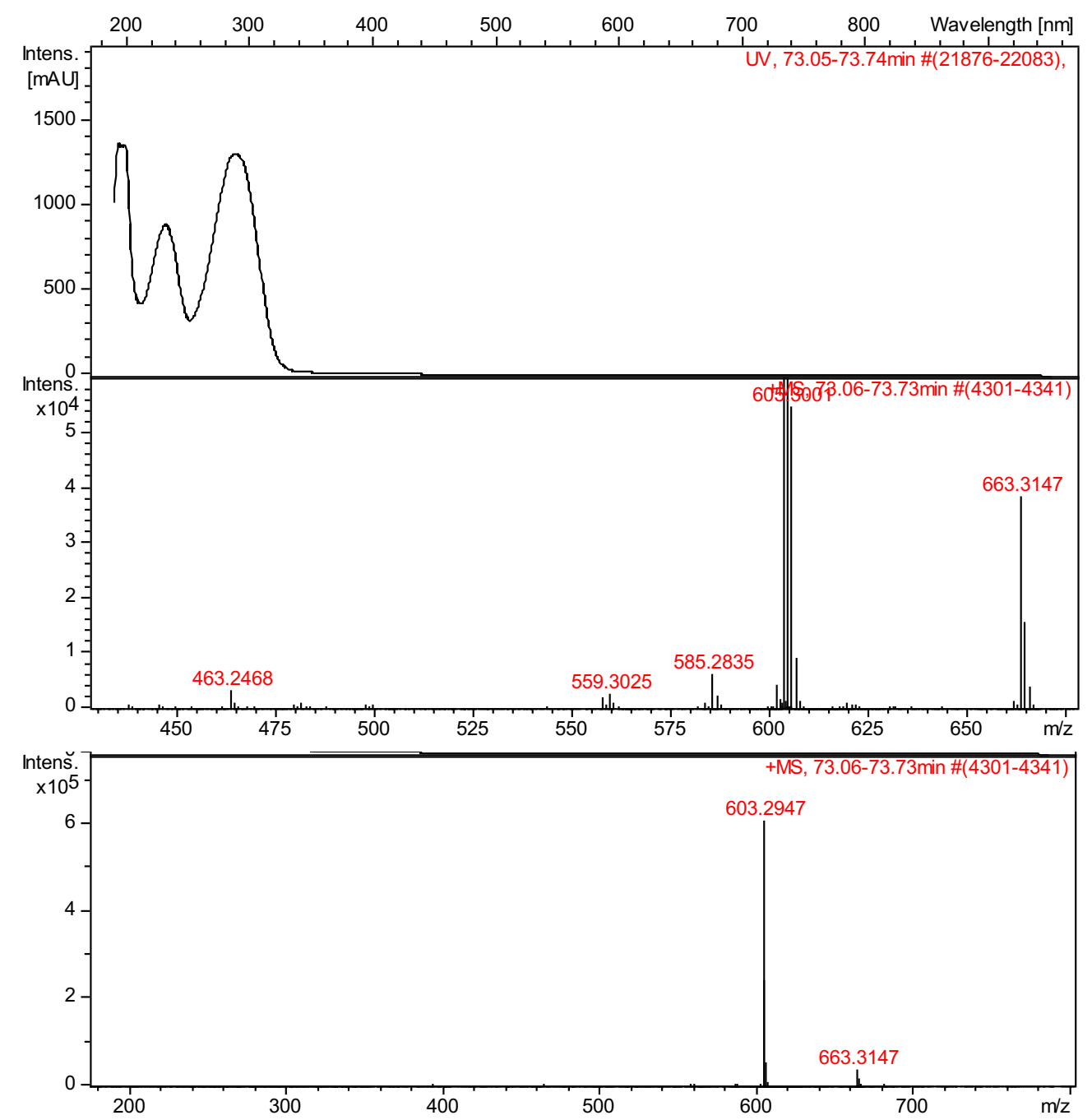


Figure S21. HRESIMS spectrum of picraviane R (18) indicating $m / z[\mathrm{M}+\mathrm{H}]^{+}=605.3100$ (calc. for $\mathrm{C}_{36} \mathrm{H}_{45} \mathrm{O}_{8}{ }^{+} 605.3109, \Delta \mathrm{M}+1.5 \mathrm{ppm}$ ), and $m / z\left[\mathrm{M}+\mathrm{H}_{-} \mathrm{C}_{6} \mathrm{H}_{5} \mathrm{CO}_{2} \mathrm{H}-\mathrm{H}_{2} \mathrm{O}\right]^{+}=465.2618$ (calc. for $\left.\mathrm{C}_{29} \mathrm{H}_{37} \mathrm{O}_{5}{ }^{+} 465.2635, \Delta \mathrm{M}+3.8 \mathrm{ppm}\right)$.

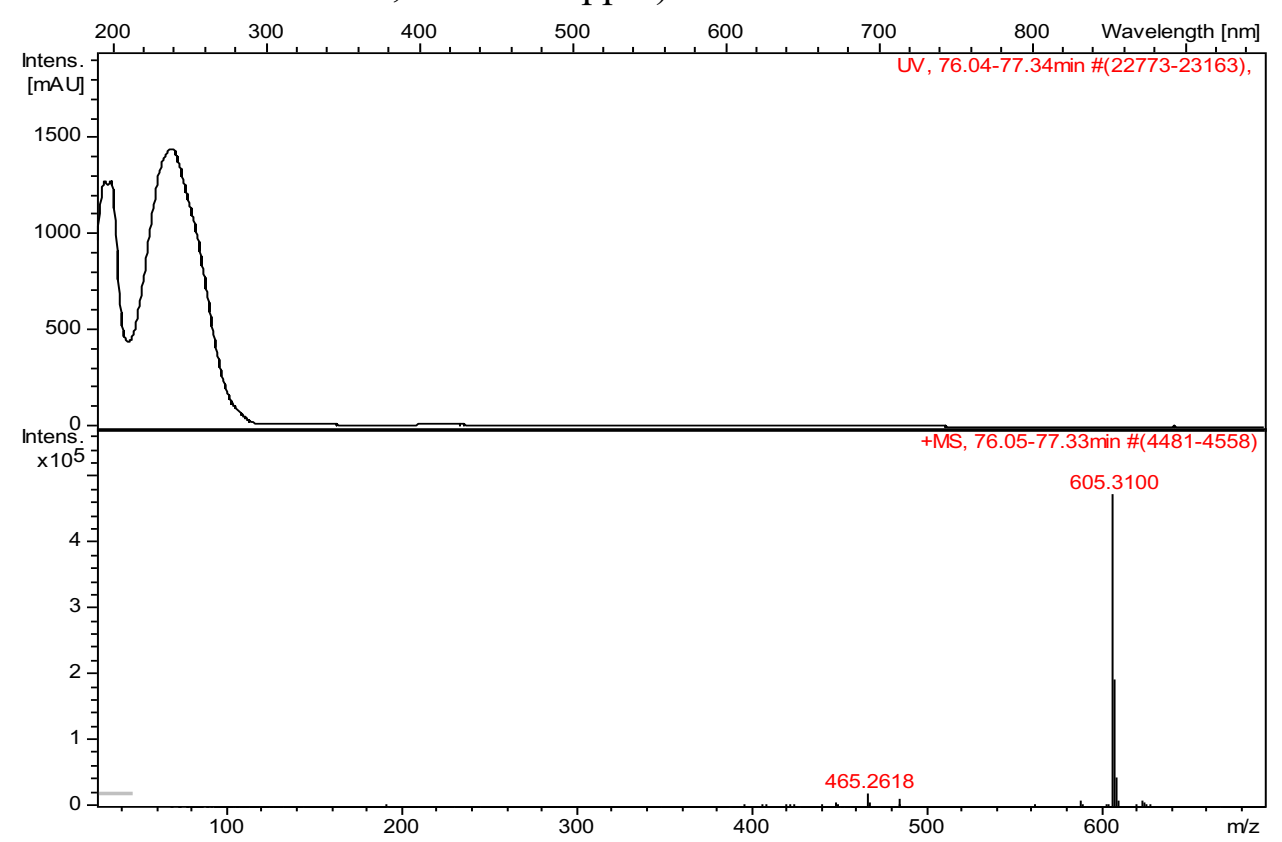

Figure S22. HRESIMS spectrum of picraviane S (19) indicating $m / z[\mathrm{M}+\mathrm{H}]^{+}=605.3099$ (calc. for $\mathrm{C}_{36} \mathrm{H}_{45} \mathrm{O}_{8}{ }^{+} 605.3109, \Delta \mathrm{M}+1.6 \mathrm{ppm}$ ) and $m / z\left[\mathrm{M}+\mathrm{H}-\mathrm{C}_{6} \mathrm{H}_{5} \mathrm{CO}_{2} \mathrm{H}-\mathrm{H}_{2} \mathrm{O}\right]^{+}=465.2621$ (calc. for $\mathrm{C}_{29} \mathrm{H}_{37} \mathrm{O}_{5}{ }^{+} 465.2635, \Delta \mathrm{M}+3.1 \mathrm{ppm}$ ).

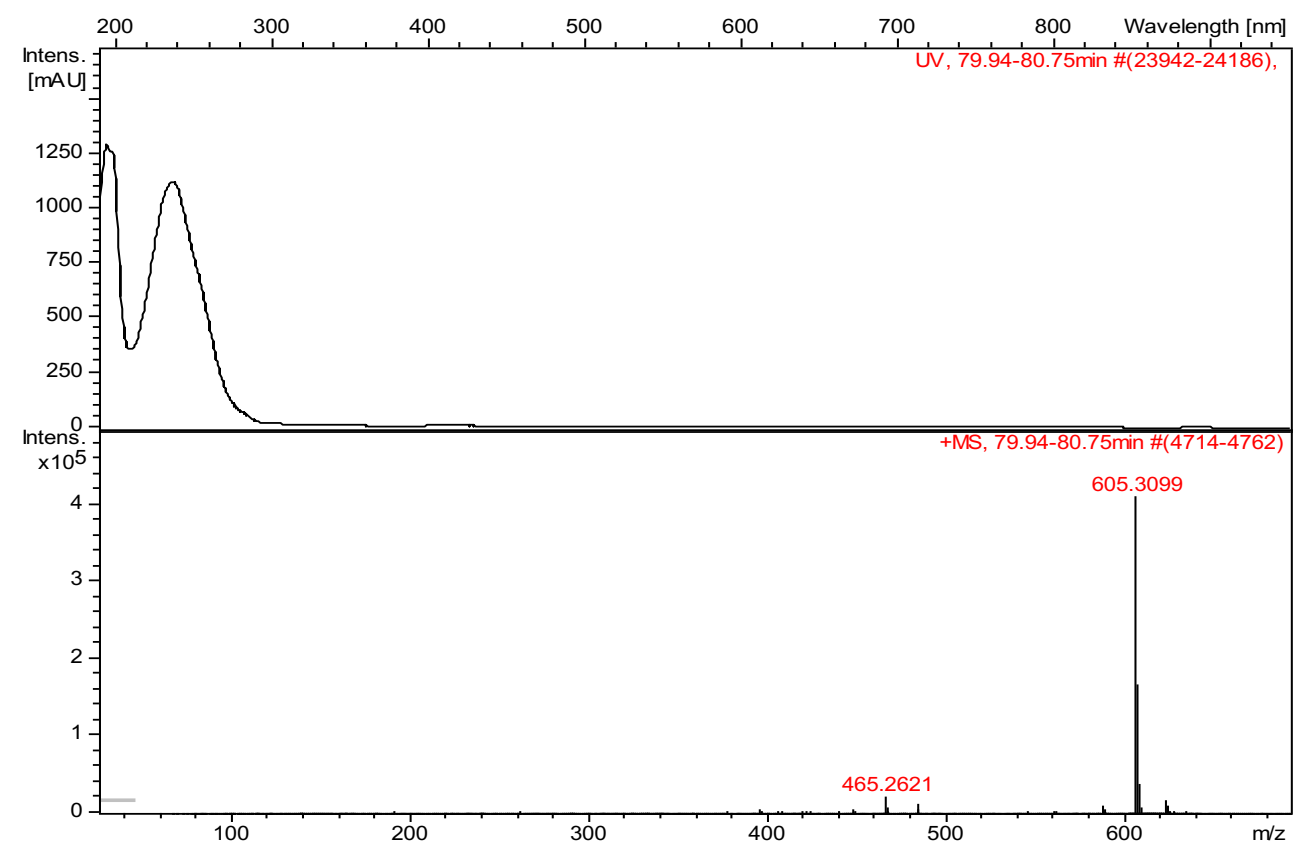


Figure S23. HRESIMS spectrum of picraviane T (21) (two different $m / z$-expansions) indicating $m / z[\mathrm{M}+\mathrm{H}]^{+}=679.3465$ (calc. for $\mathrm{C}_{39} \mathrm{H}_{51} \mathrm{O}_{10}{ }^{+}$679.3476, $\Delta \mathrm{M}+1.7 \mathrm{ppm}$ ), $\quad m / z[\mathrm{M}+\mathrm{H}-$ $\left.\mathrm{CH}_{3} \mathrm{CO}_{2} \mathrm{H}\right]^{+}=619.3277$ (calc. for $\mathrm{C}_{37} \mathrm{H}_{47} \mathrm{O}_{8}{ }^{+} 619.3265, \Delta \mathrm{M}-1.9 \mathrm{ppm}$ ), $m / z\left[\mathrm{M}+\mathrm{H}-\mathrm{CH}_{3} \mathrm{CO}_{2} \mathrm{H}-\right.$ $\left.\mathrm{CH}_{3} \mathrm{OH}\right]^{+}=587.2997$ (calc. for $\mathrm{C}_{36} \mathrm{H}_{43} \mathrm{O}_{7}{ }^{+} 587.3003, \Delta \mathrm{M}+1.1 \mathrm{ppm}$ ), and $m / z\left[\mathrm{M}+\mathrm{H}_{-} \mathrm{CH}_{3} \mathrm{CO}_{2} \mathrm{H}-\right.$ $\left.\mathrm{CH}_{3} \mathrm{OH}-\mathrm{C}_{6} \mathrm{H}_{5} \mathrm{CO}_{2} \mathrm{H}\right]^{+}=465.2627$ (calc. for $\mathrm{C}_{29} \mathrm{H}_{37} \mathrm{O}_{5}{ }^{+} 465.2635, \Delta \mathrm{M}+1.8 \mathrm{ppm}$ ).

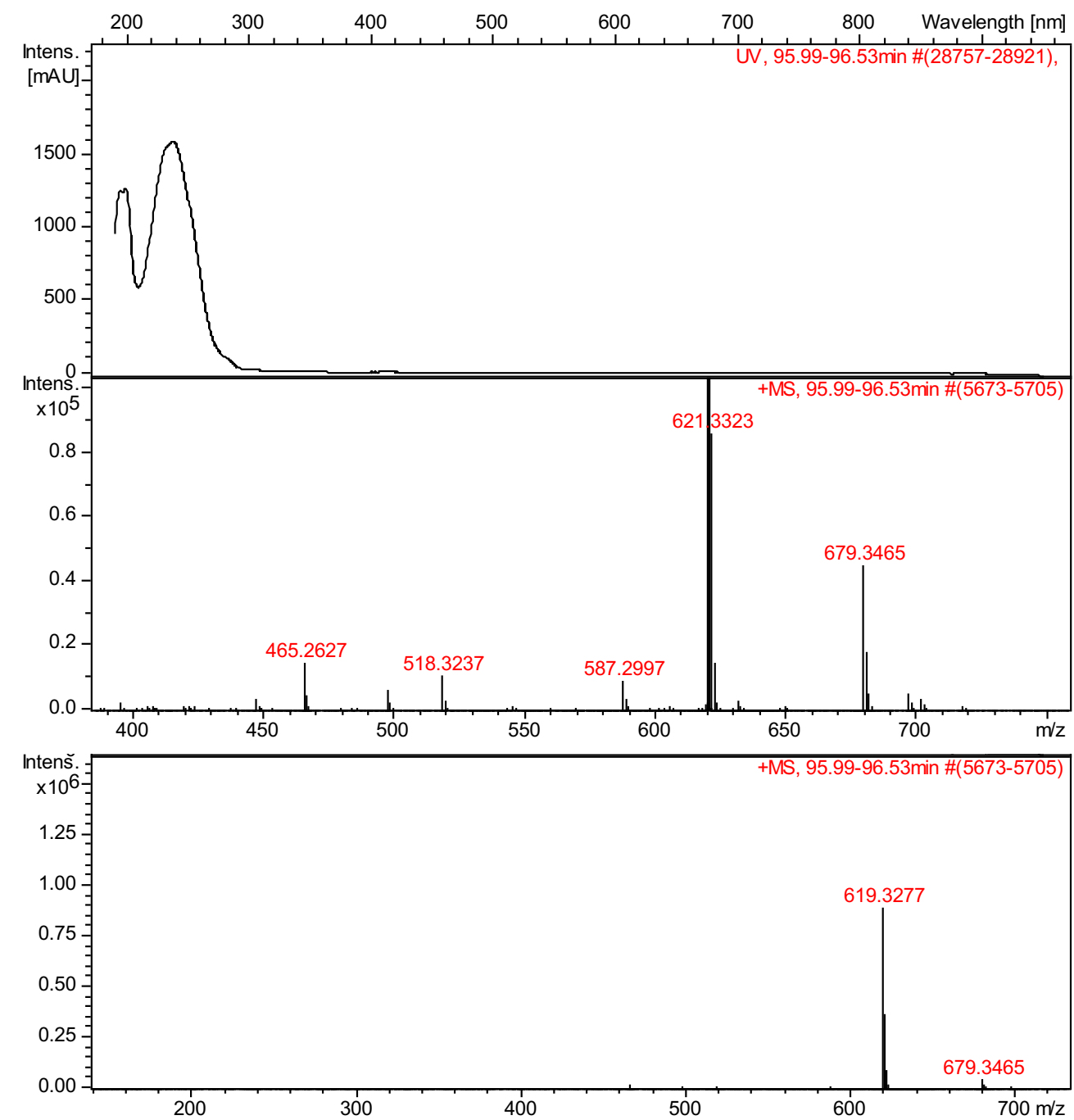


Figure S24. ${ }^{1} \mathrm{H}$ NMR spectrum of picraviane $\mathrm{G}(6)$ in $\mathrm{CDCl}_{3}(600 \mathrm{MHz})$.

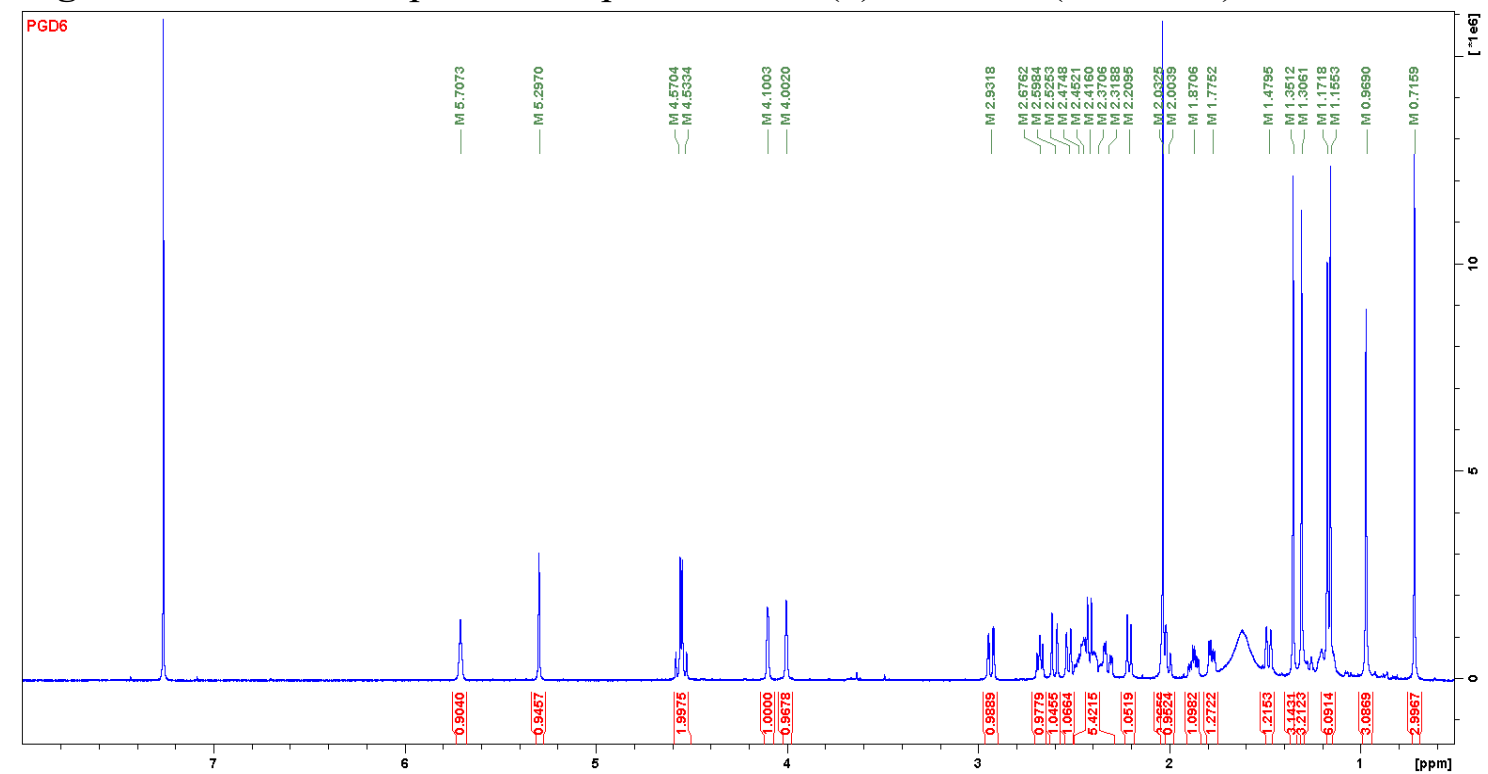

Figure S25. Expansion of the ${ }^{1} \mathrm{H}$ NMR spectrum of picraviane $\mathrm{G}(6)$ in $\mathrm{CDCl}_{3}(600 \mathrm{MHz})$.

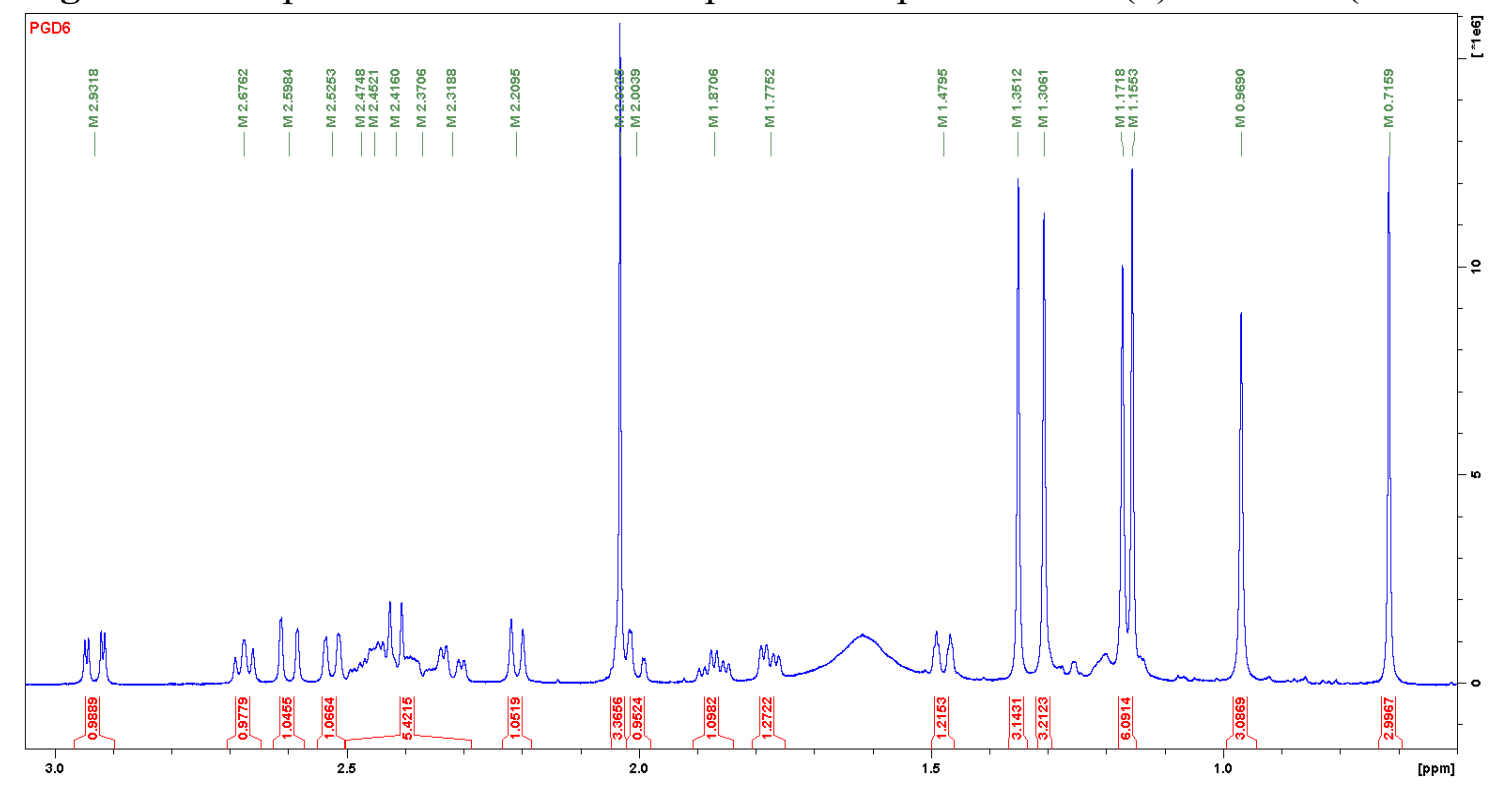


Figure S26. ${ }^{13} \mathrm{C}$ NMR spectrum of picraviane $\mathrm{G}(6)$ in $\mathrm{CDCl}_{3}(150 \mathrm{MHz})$.

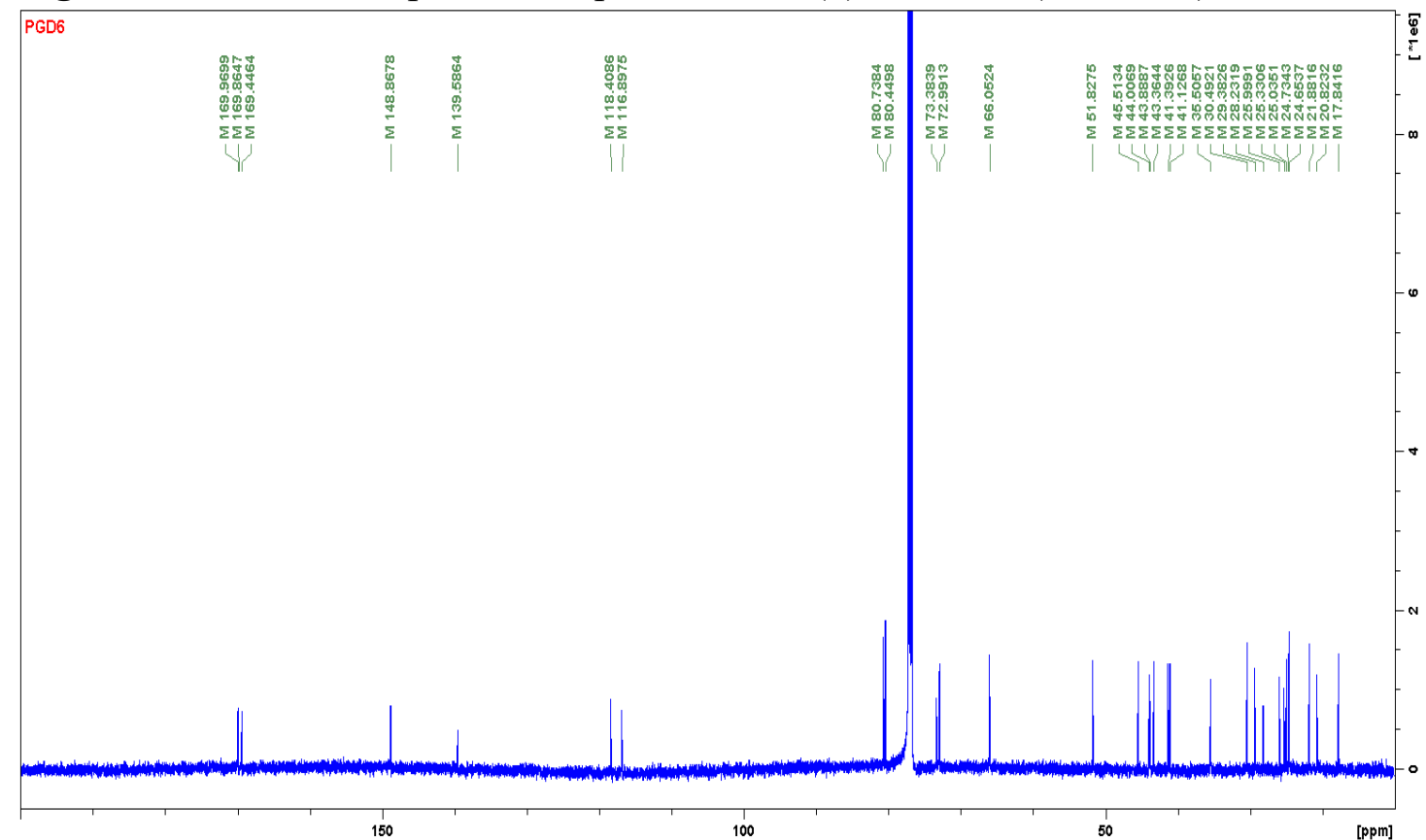

Figure S27. HSQC spectrum of picraviane $\mathrm{G}(6)$ in $\mathrm{CDCl}_{3}(600 \mathrm{MHz})$.

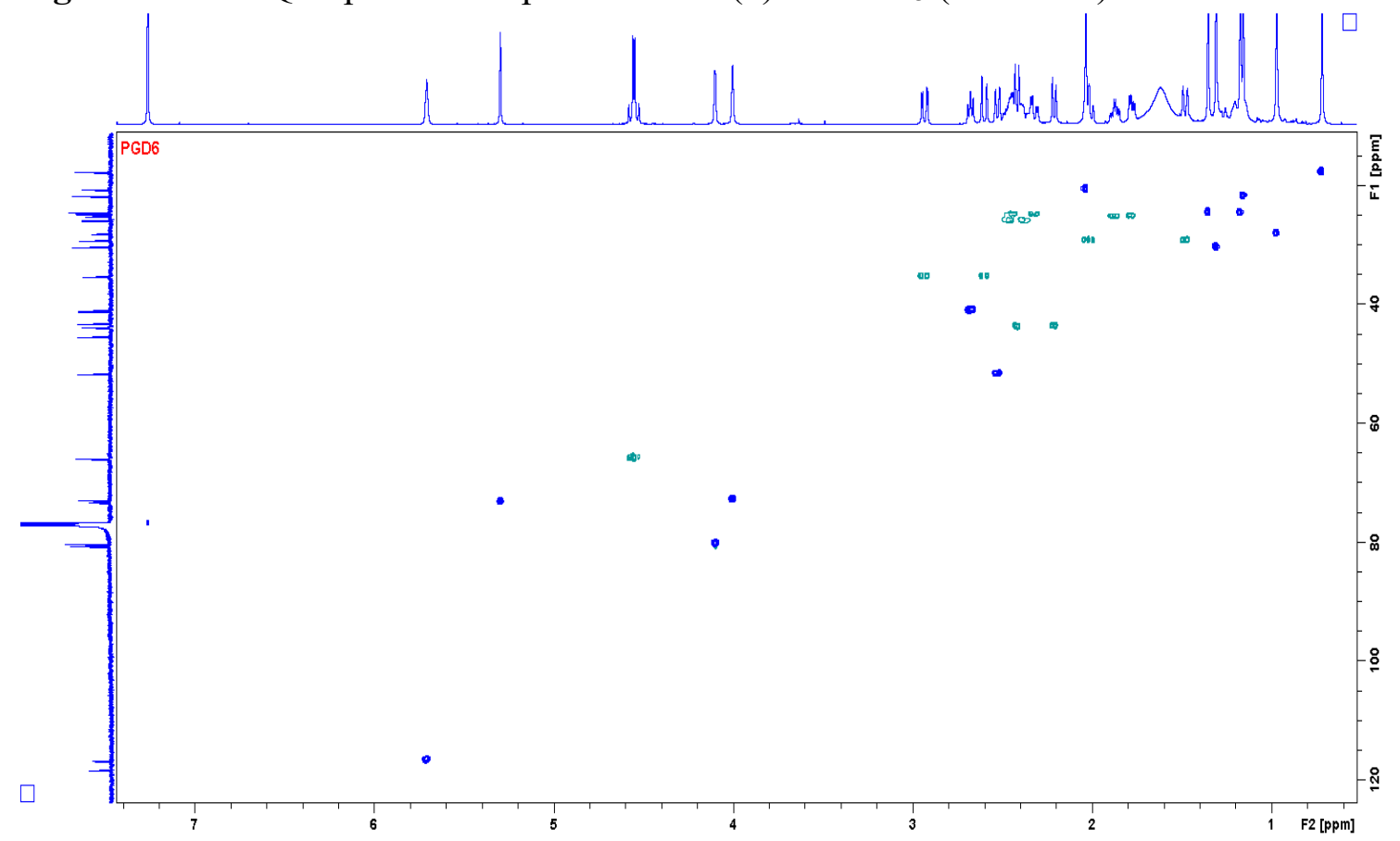


Figure S28. HMBC spectrum of picraviane $\mathrm{G}(6)$ in $\mathrm{CDCl}_{3}(600 \mathrm{MHz})$.

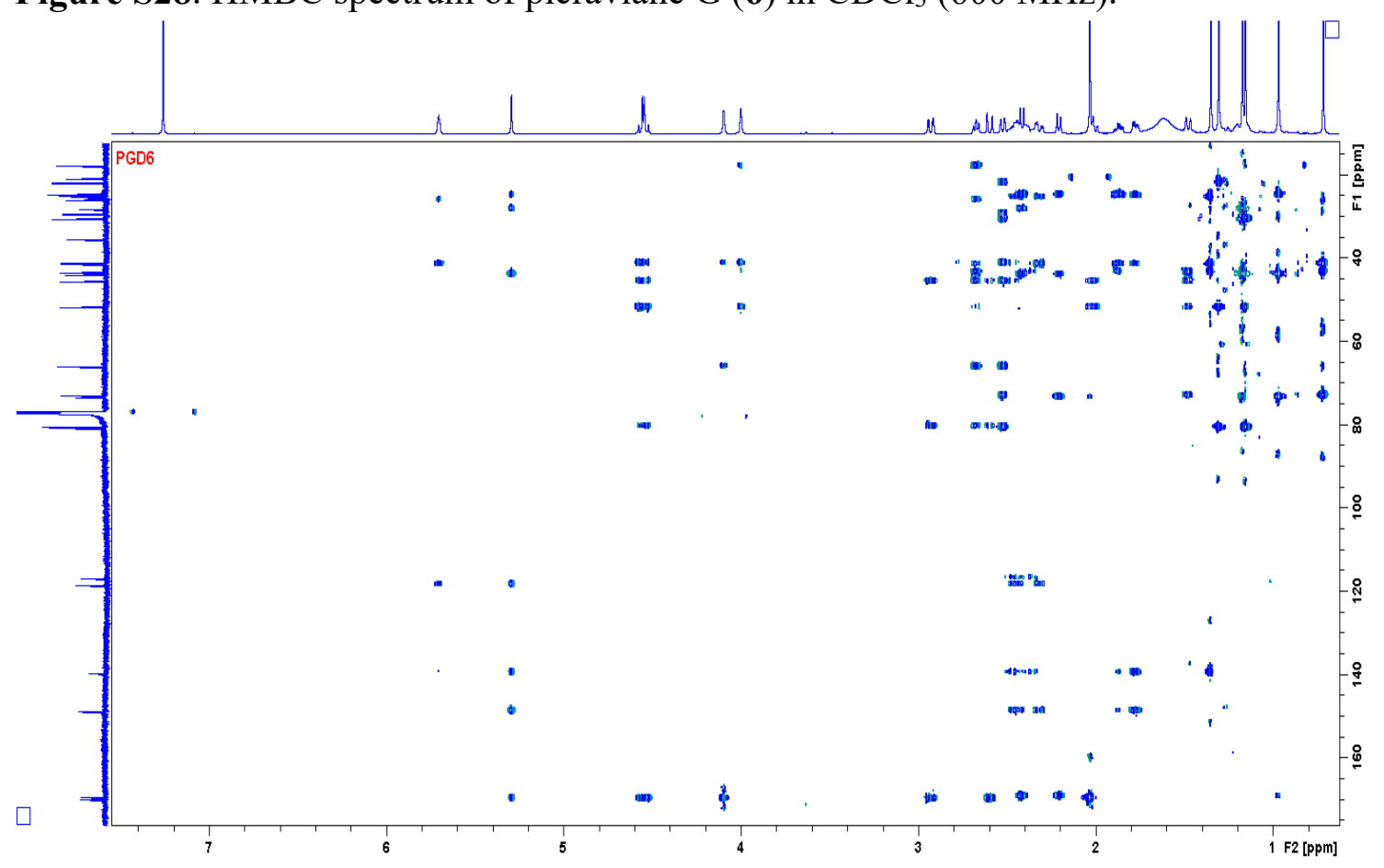

Figure S29. COSY spectrum of picraviane $\mathrm{G}(\mathbf{6})$ in $\mathrm{CDCl}_{3}(600 \mathrm{MHz})$.

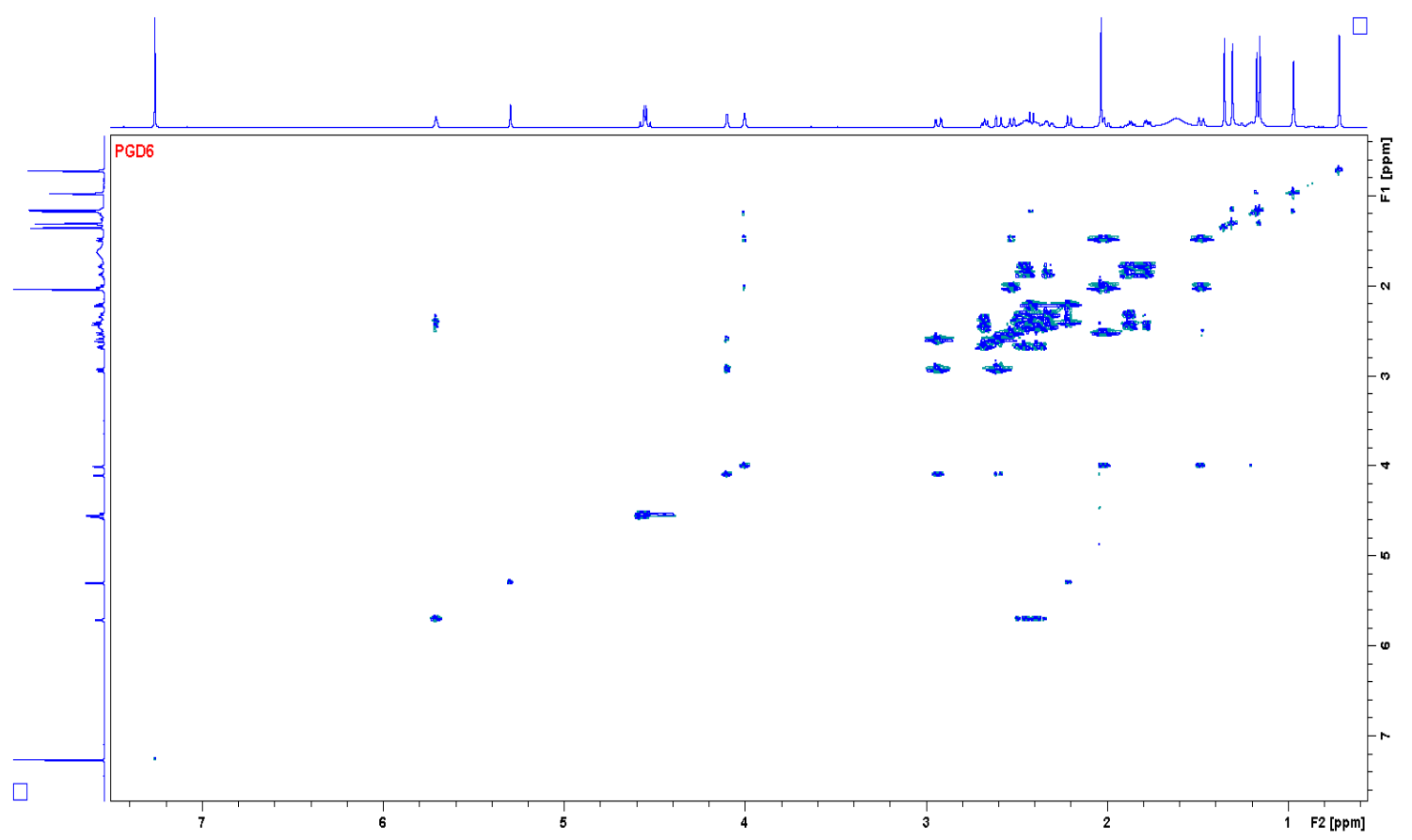

26 
Figure S30. NOESY spectrum of picraviane $\mathrm{G}(6)$ in $\mathrm{CDCl}_{3}(600 \mathrm{MHz})$.

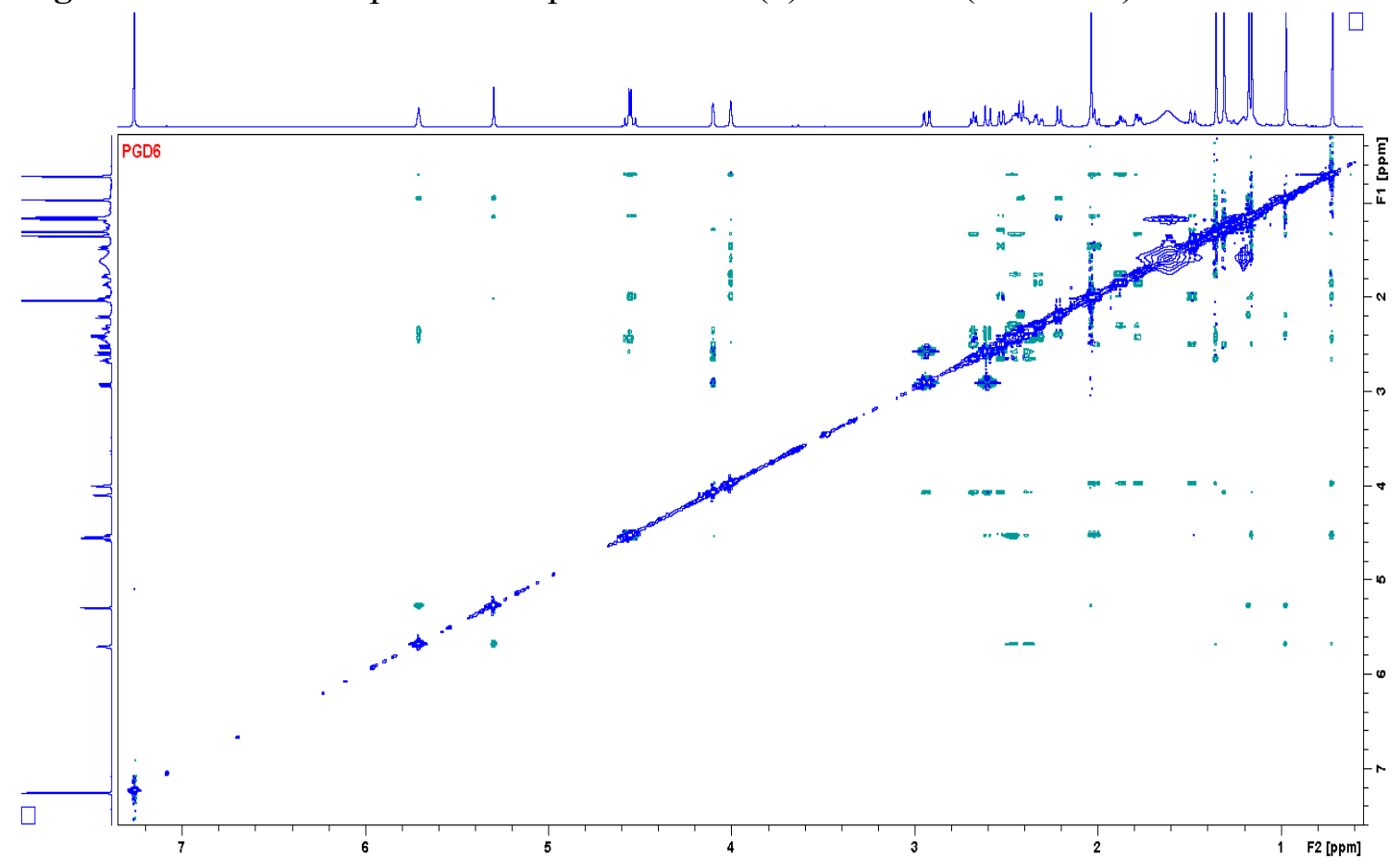

Figure S31. ${ }^{1} \mathrm{H}$ NMR spectrum of picraviane J (9) in $\mathrm{CDCl}_{3}(600 \mathrm{MHz})$.

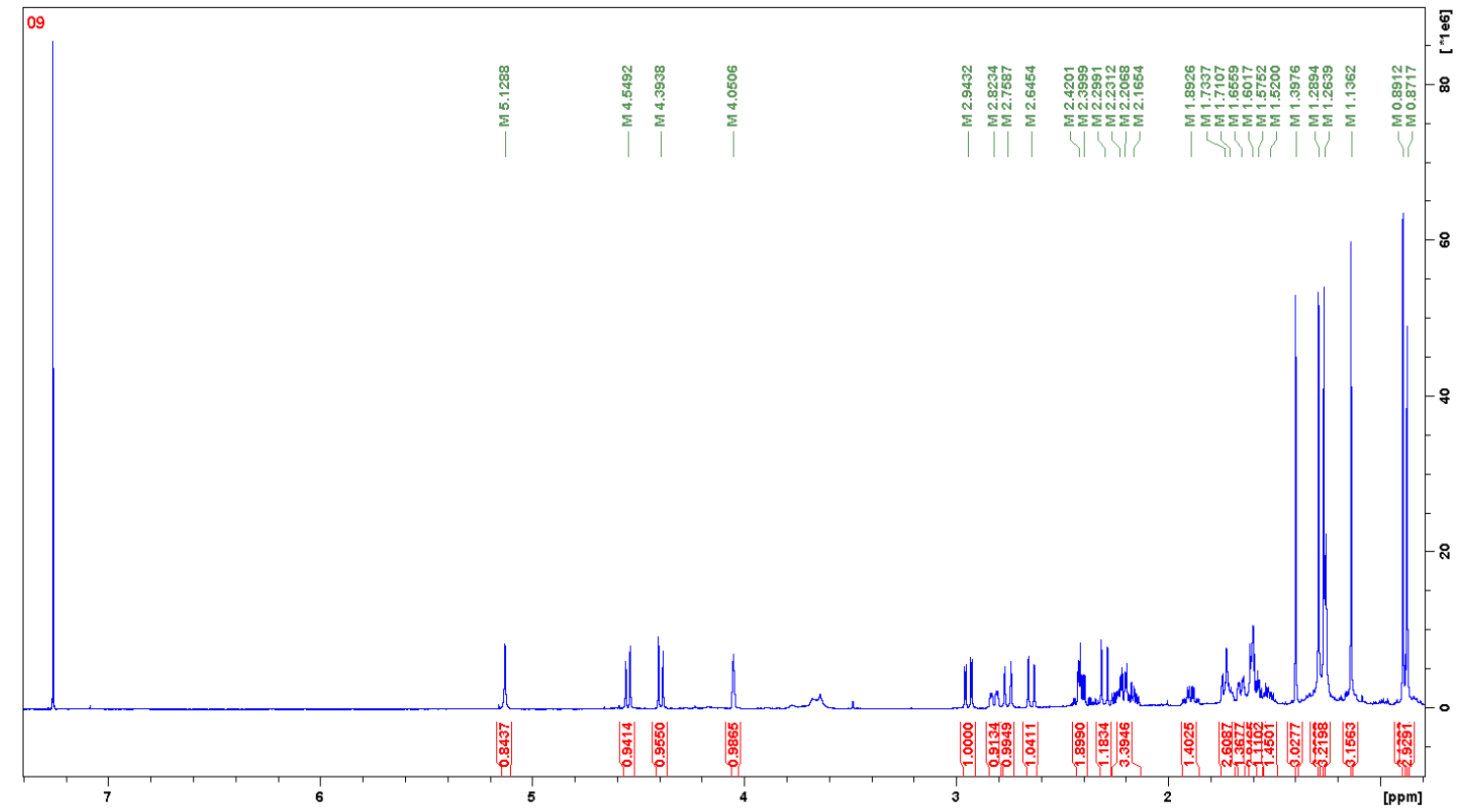


Figure S32. Expansion of the ${ }^{1} \mathrm{H}$ NMR spectrum of picraviane $\mathrm{J}(9)$ in $\mathrm{CDCl}_{3}(600 \mathrm{MHz})$.

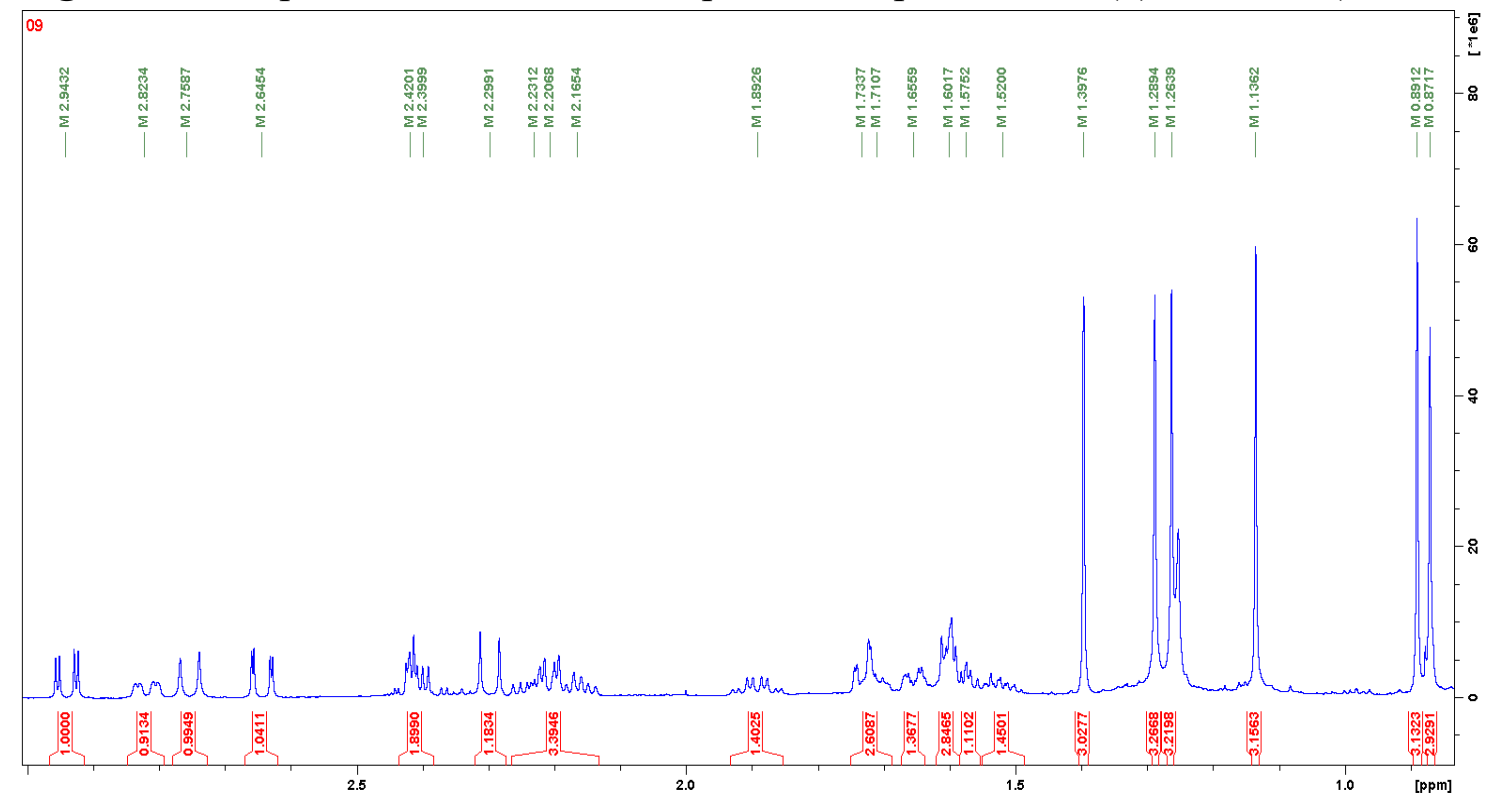

Figure S33. ${ }^{13} \mathrm{C}$ NMR spectrum of picraviane $\mathrm{J}(9)$ in $\mathrm{CDCl}_{3}(150 \mathrm{MHz})$.

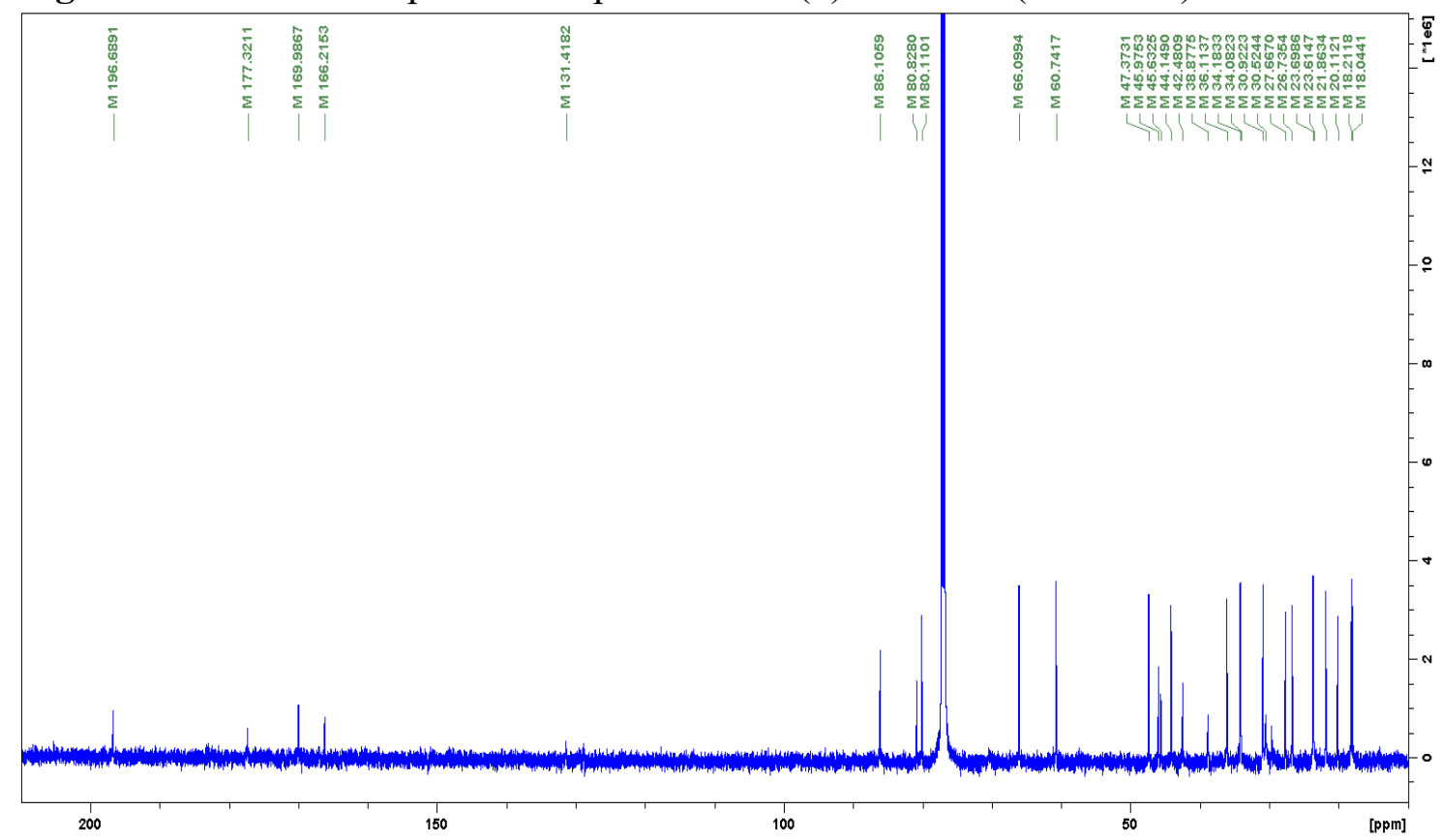


Figure S34. HSQC spectrum of picraviane J (9) in $\mathrm{CDCl}_{3}(600 \mathrm{MHz})$.

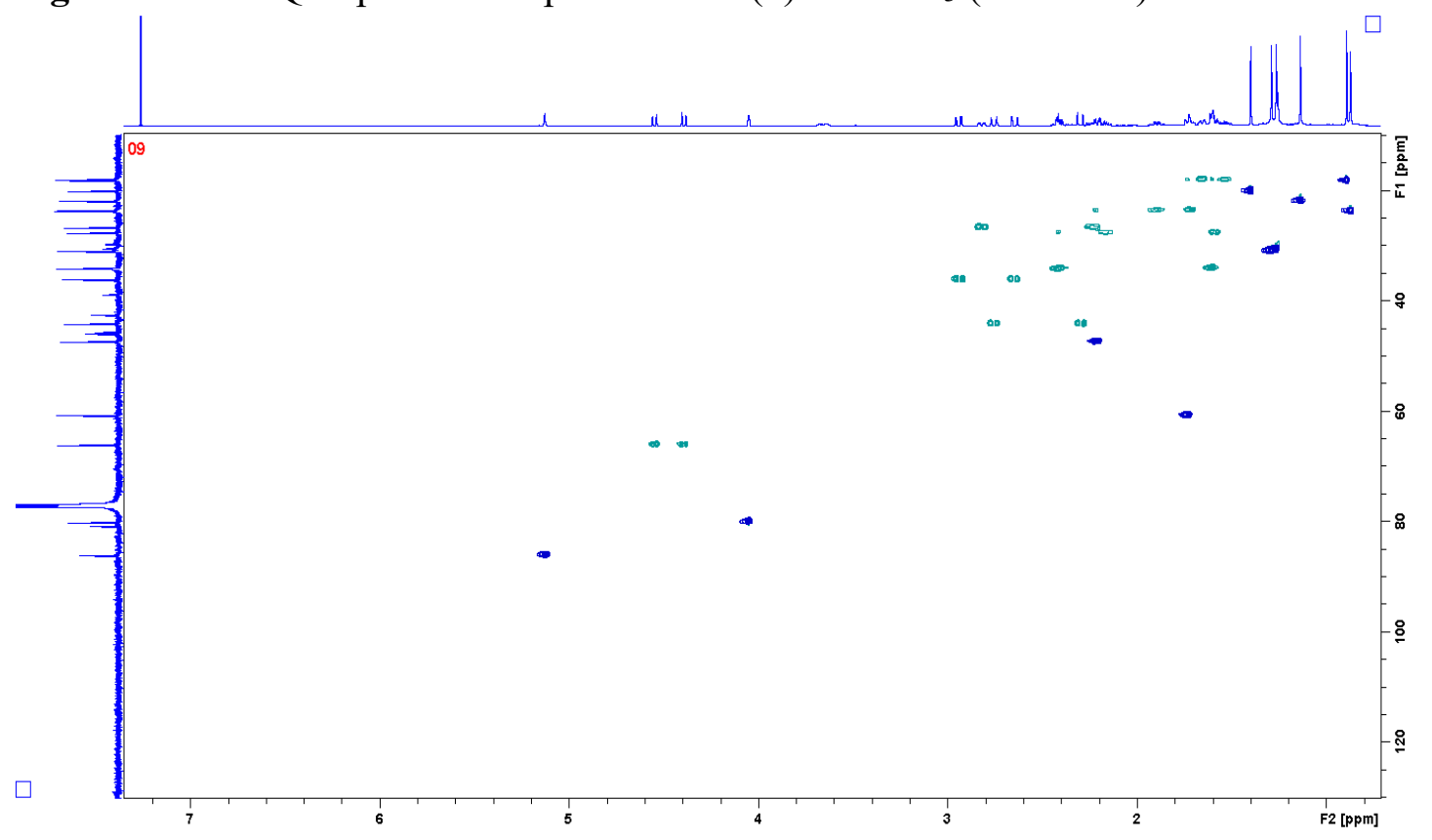

Figure S35. HMBC spectrum of picraviane $\mathrm{J}(9)$ in $\mathrm{CDCl}_{3}(600 \mathrm{MHz})$.

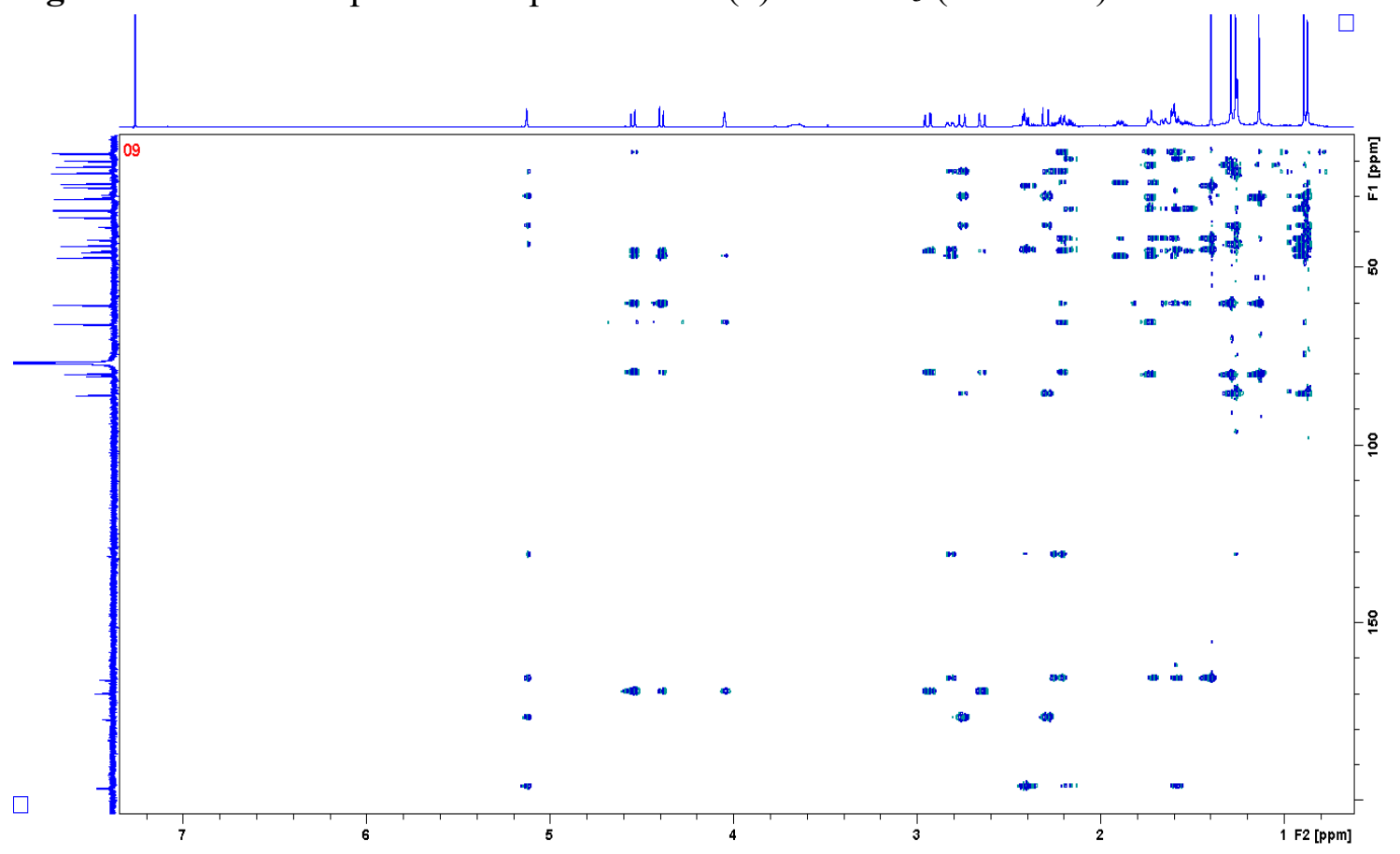

29 
Figure S36. COSY spectrum of picraviane $\mathrm{J}(9)$ in $\mathrm{CDCl}_{3}(600 \mathrm{MHz})$.

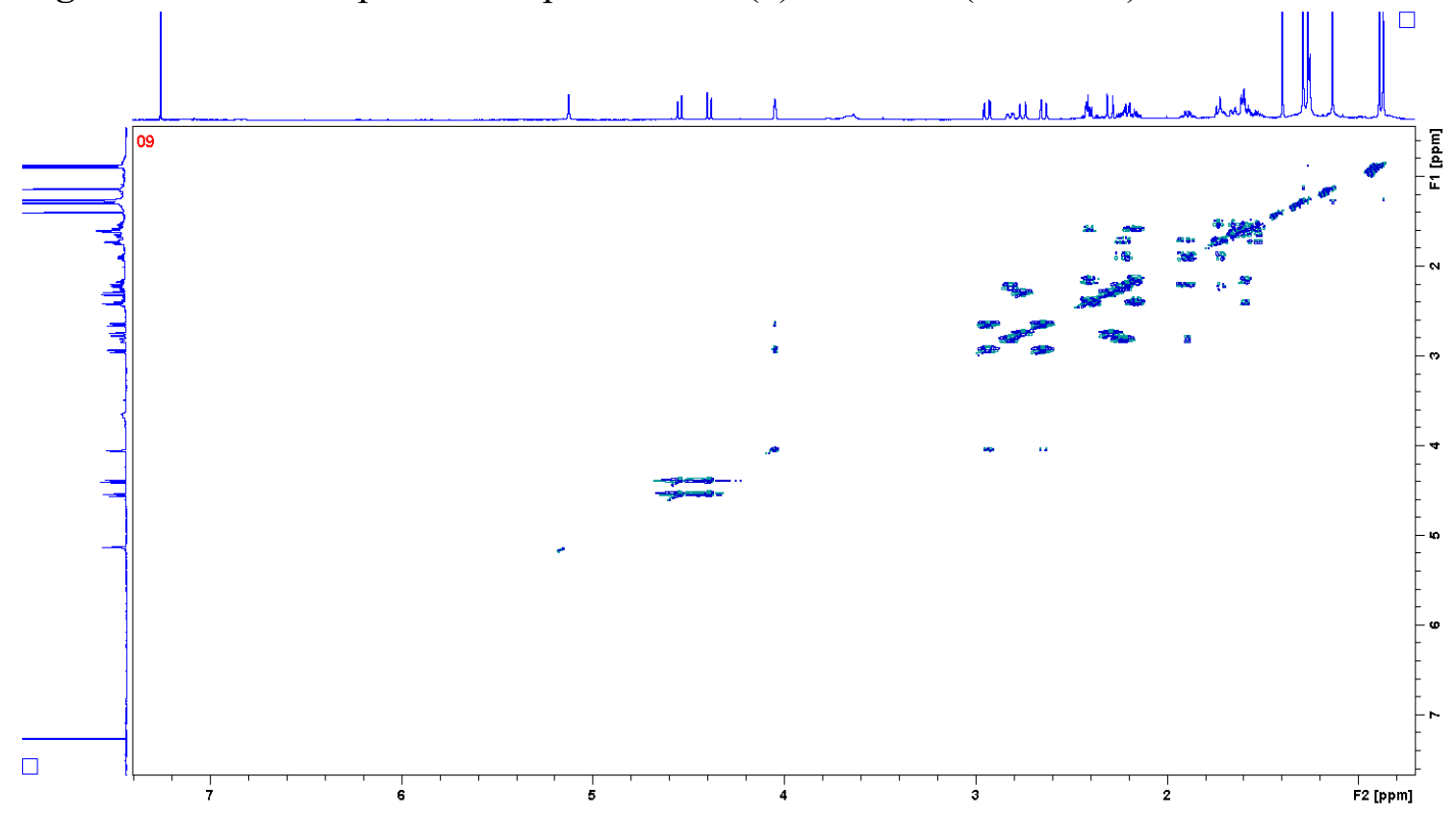

Figure S37. NOESY spectrum of picraviane J (9) in $\mathrm{CDCl}_{3}(600 \mathrm{MHz})$.

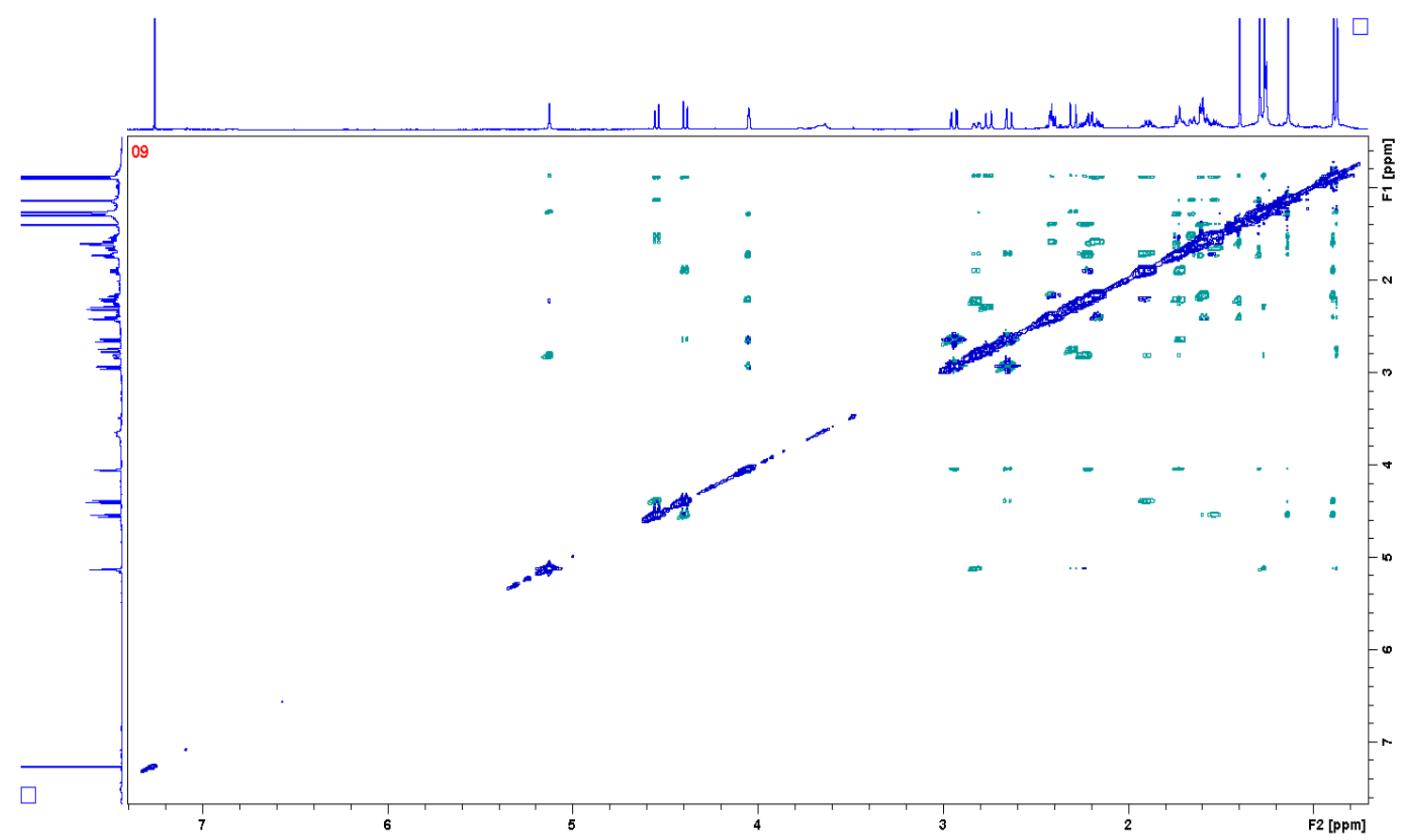


Figure S38. Expansion of the NOESY spectrum of picraviane $\mathrm{J}(9)$ in $\mathrm{CDCl}_{3}(600 \mathrm{MHz})$.

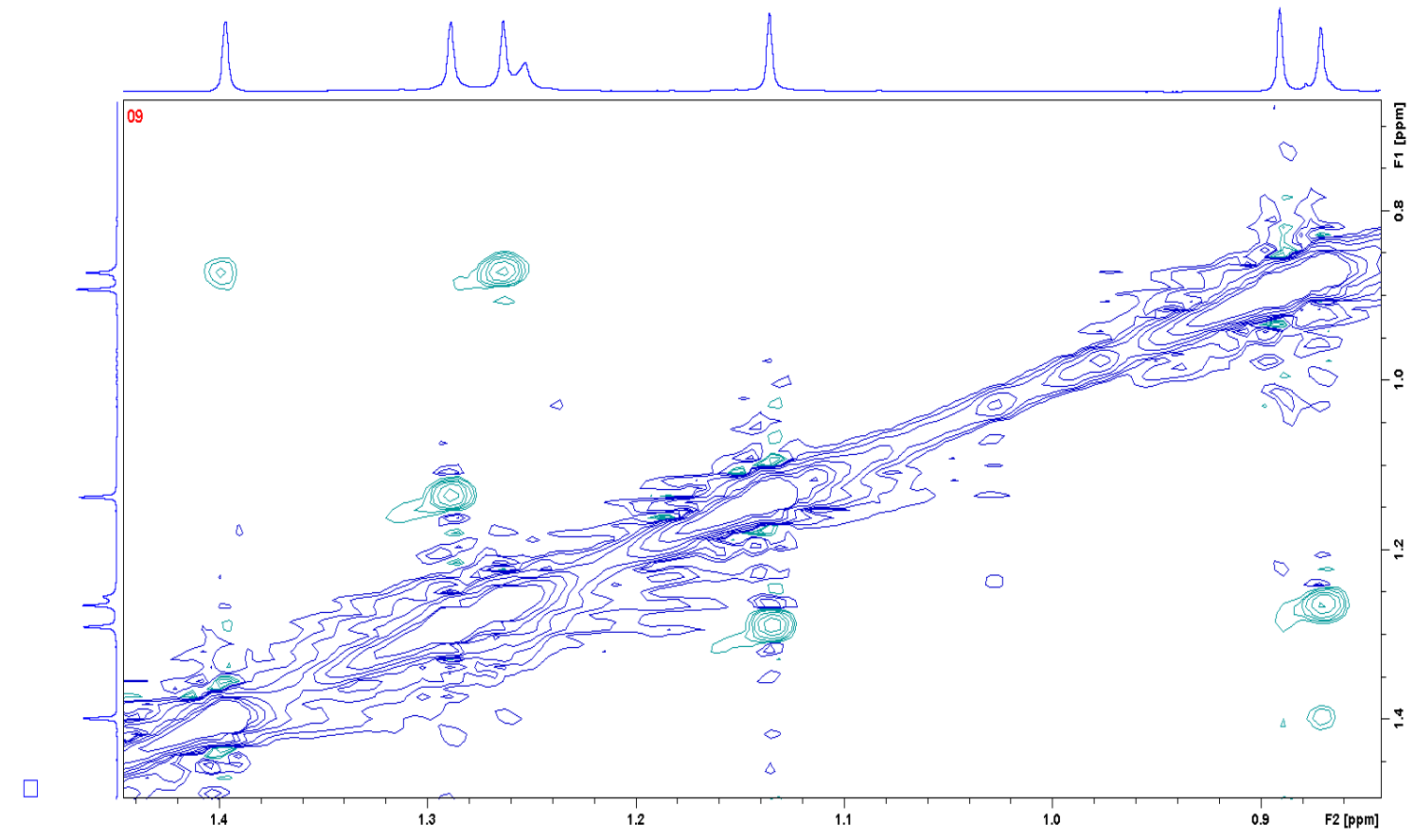

Figure S39. ${ }^{1} \mathrm{H}$ NMR spectrum of picraviane $\mathrm{K}(\mathbf{1 0})$ in $\mathrm{CDCl}_{3}(600 \mathrm{MHz})$.

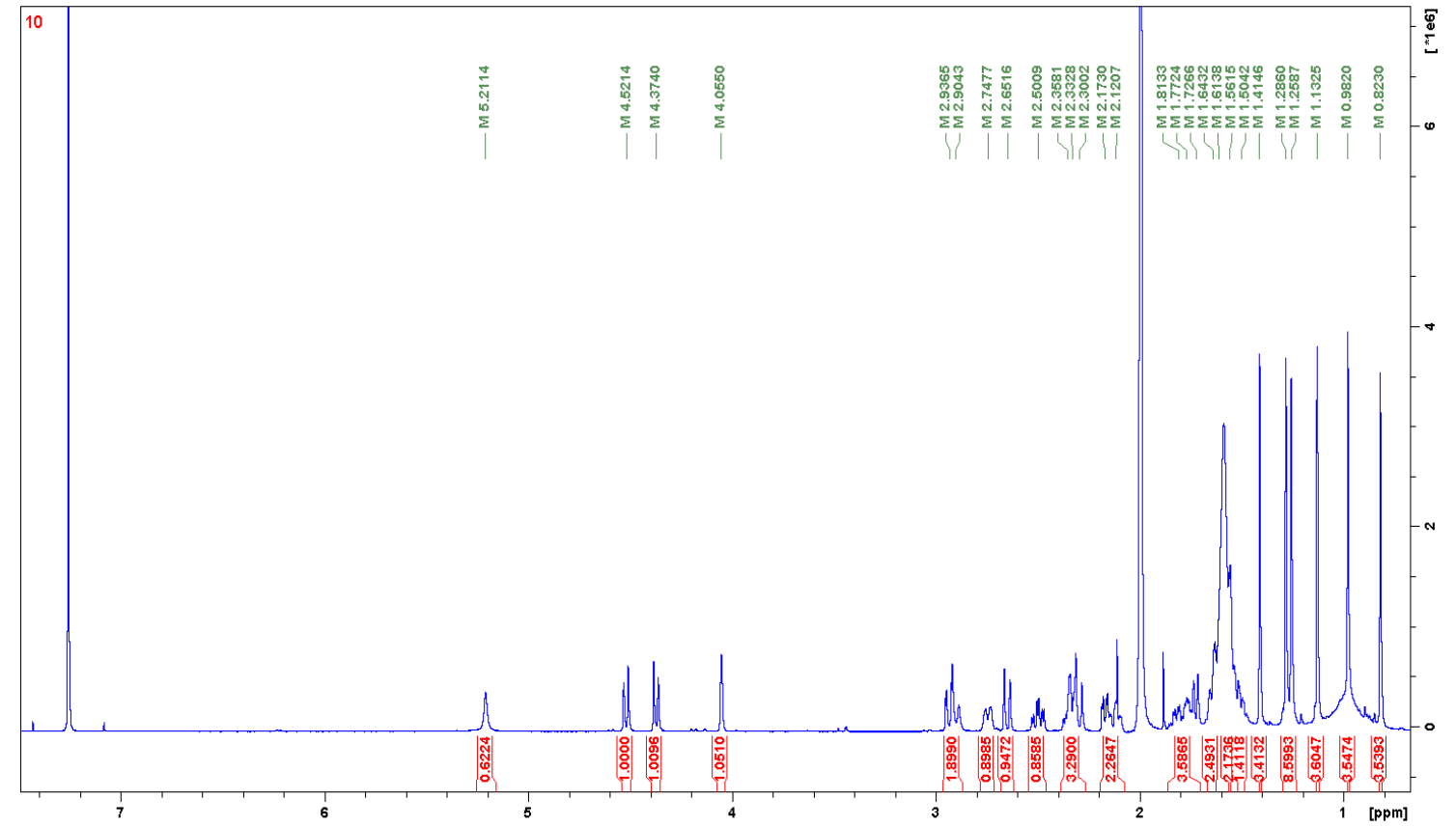


Figure S40. Expansion of the ${ }^{1} \mathrm{H}$ NMR spectrum of picraviane $\mathrm{K}(\mathbf{1 0})$ in $\mathrm{CDCl}_{3}(600 \mathrm{MHz})$.

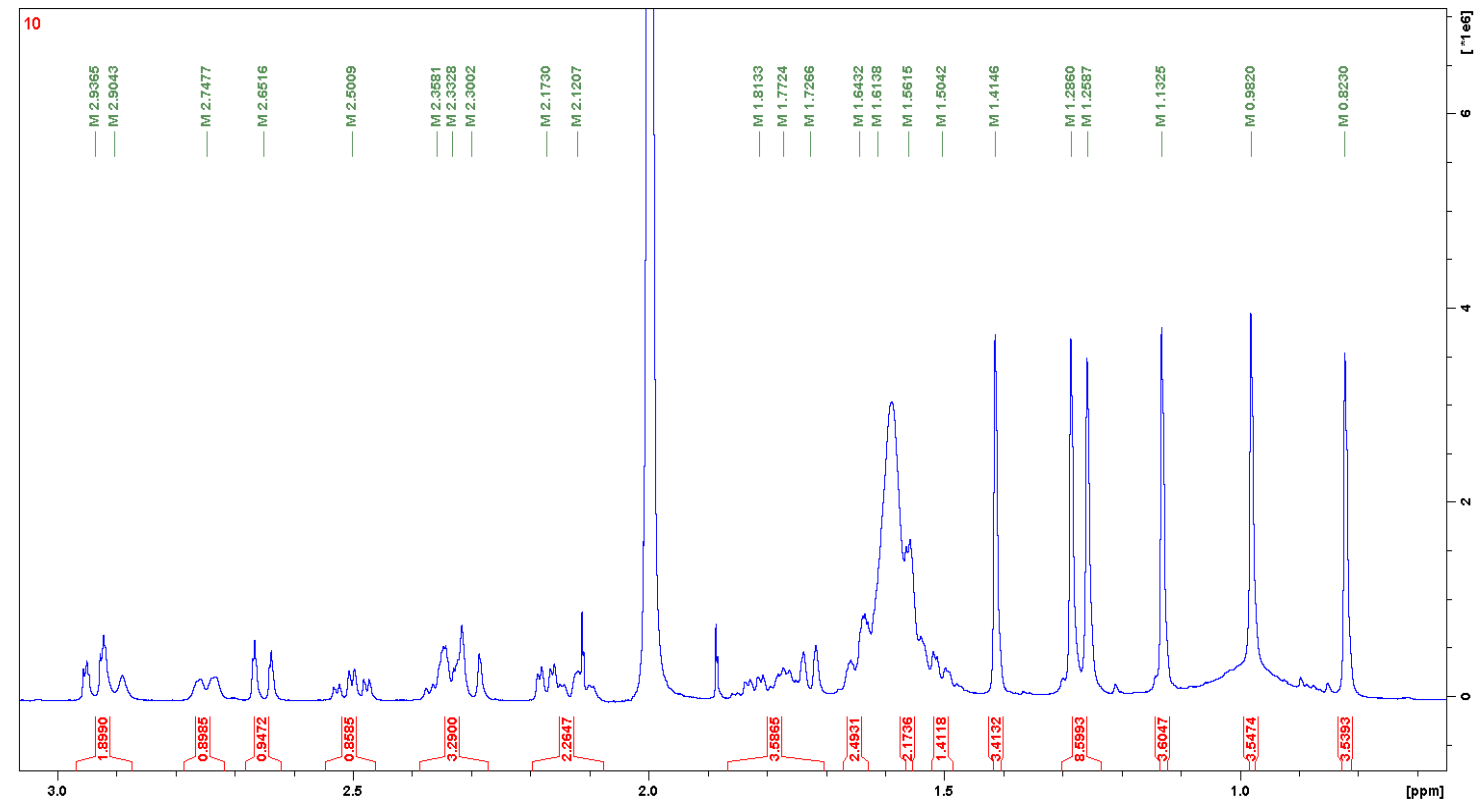

Figure S41. ${ }^{13} \mathrm{C}$ NMR spectrum of picraviane $\mathrm{K}(\mathbf{1 0})$ in $\mathrm{CDCl}_{3}(150 \mathrm{MHz})$.

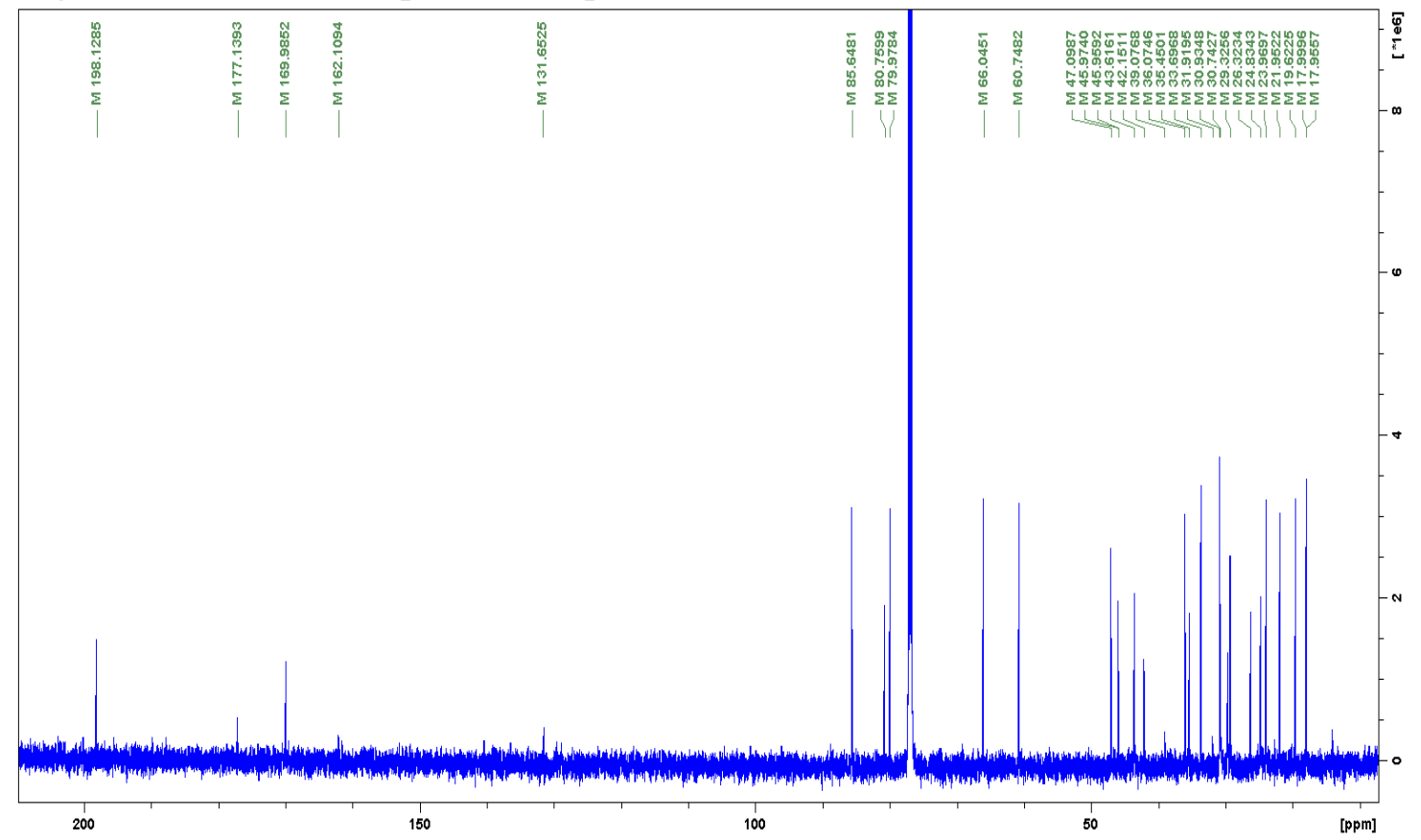


Figure S42. HSQC spectrum of picraviane $\mathrm{K}(\mathbf{1 0})$ in $\mathrm{CDCl}_{3}(600 \mathrm{MHz})$.

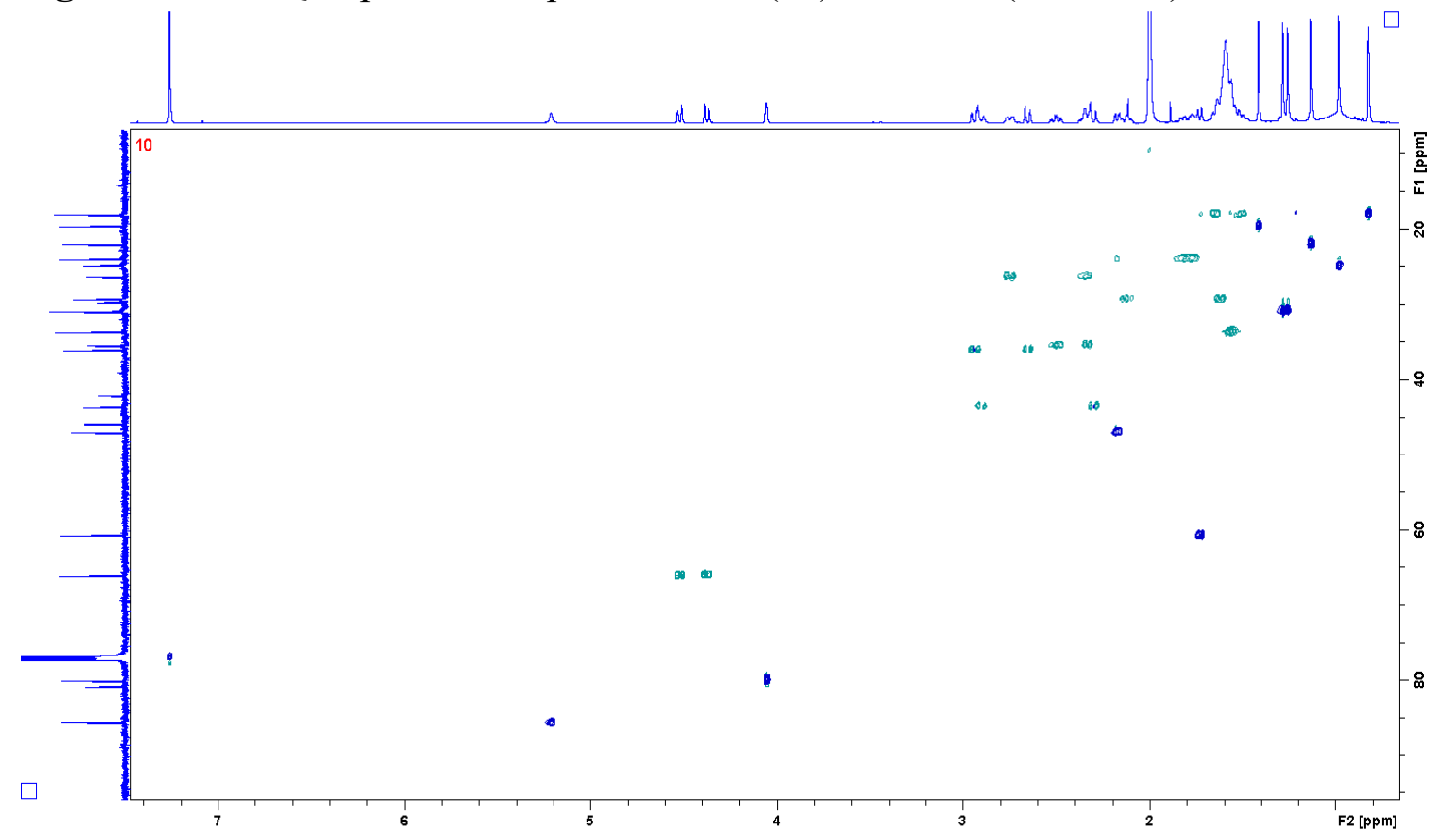

Figure S43. HMBC spectrum of picraviane $\mathrm{K}(\mathbf{1 0})$ in $\mathrm{CDCl}_{3}(600 \mathrm{MHz})$.

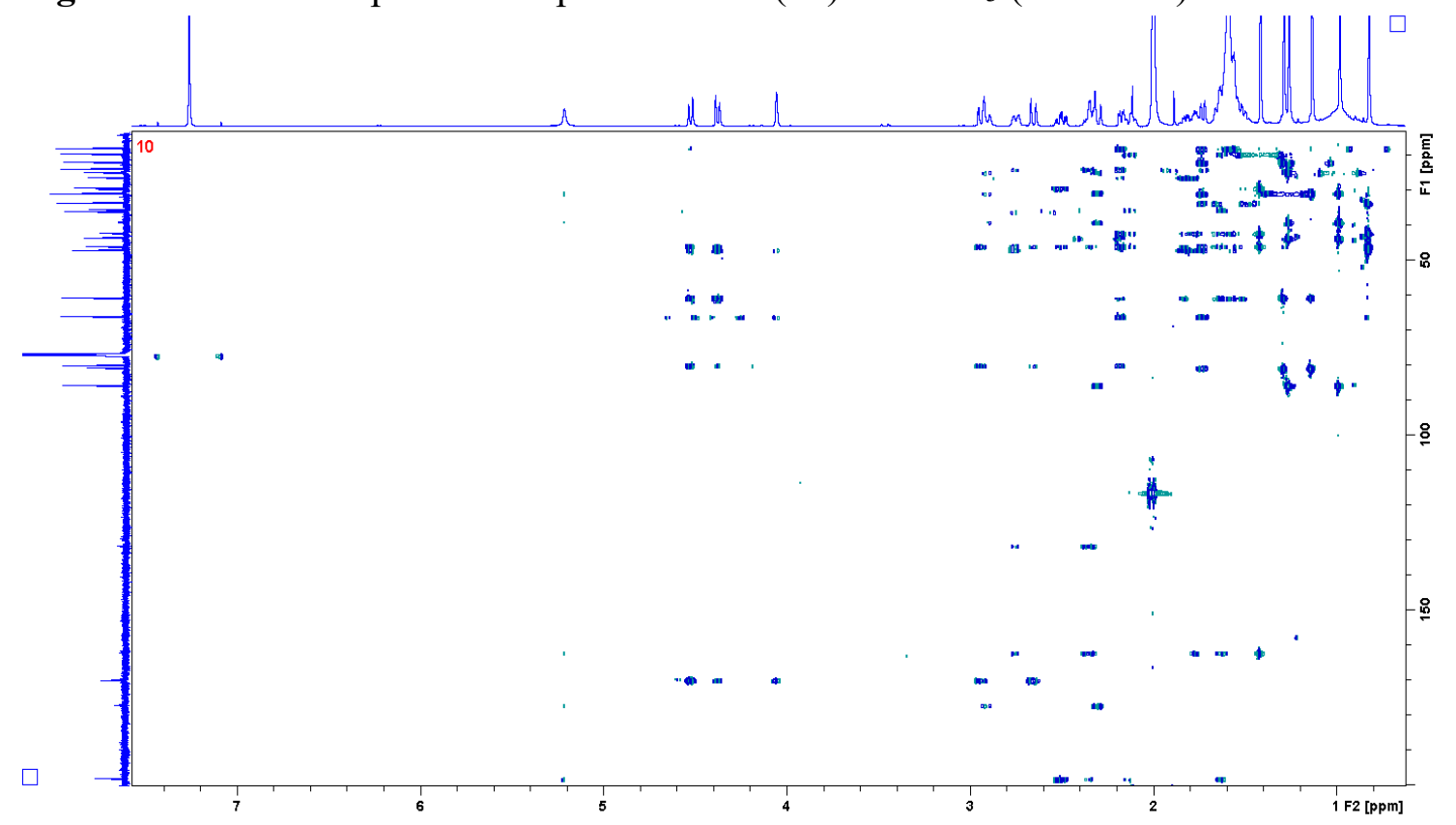


Figure S44. COSY spectrum of picraviane K (10) in $\mathrm{CDCl}_{3}(600 \mathrm{MHz})$.

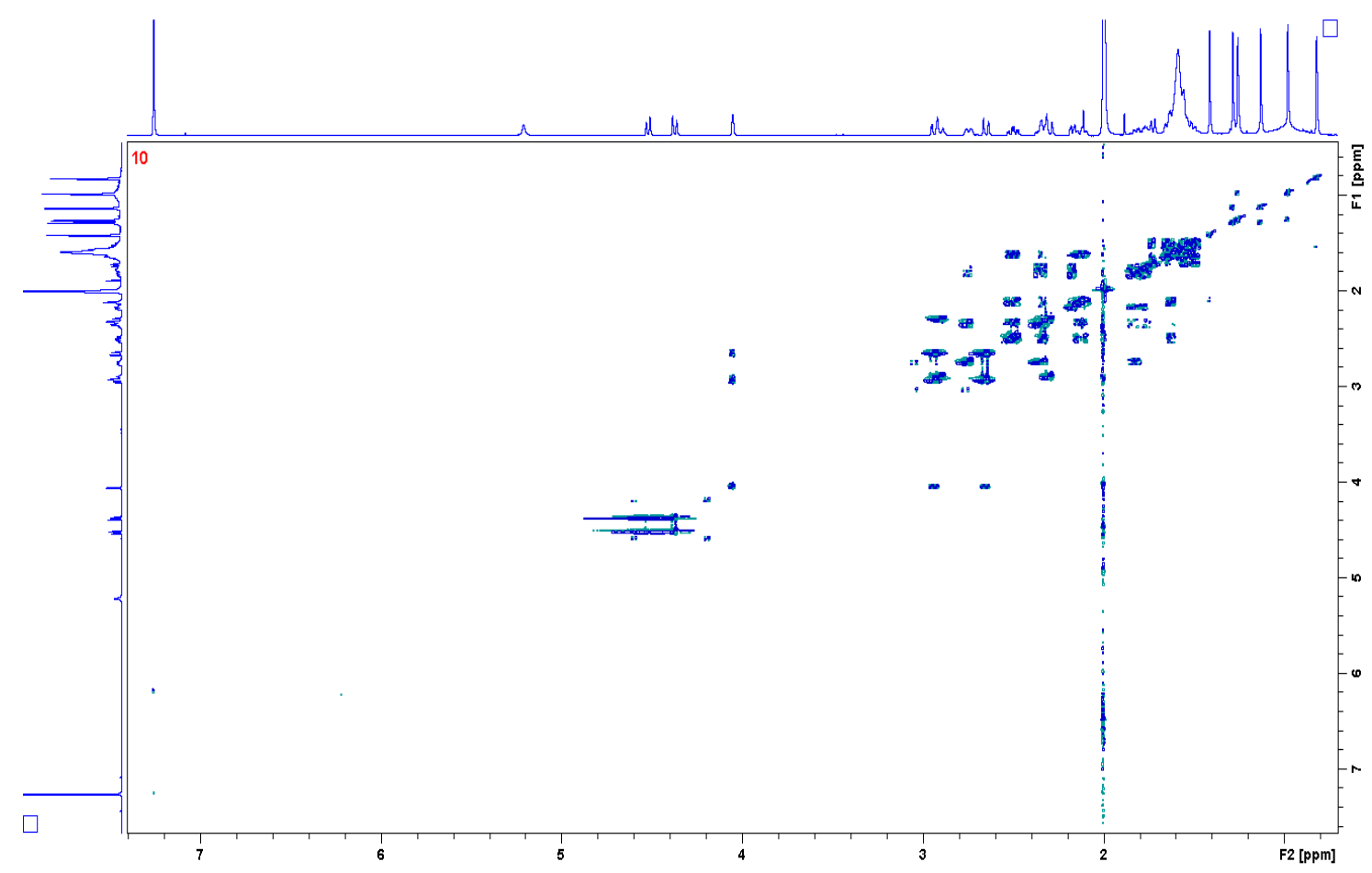

Figure S45. NOESY spectrum of picraviane $\mathrm{K}(\mathbf{1 0})$ in $\mathrm{CDCl}_{3}(600 \mathrm{MHz})$.

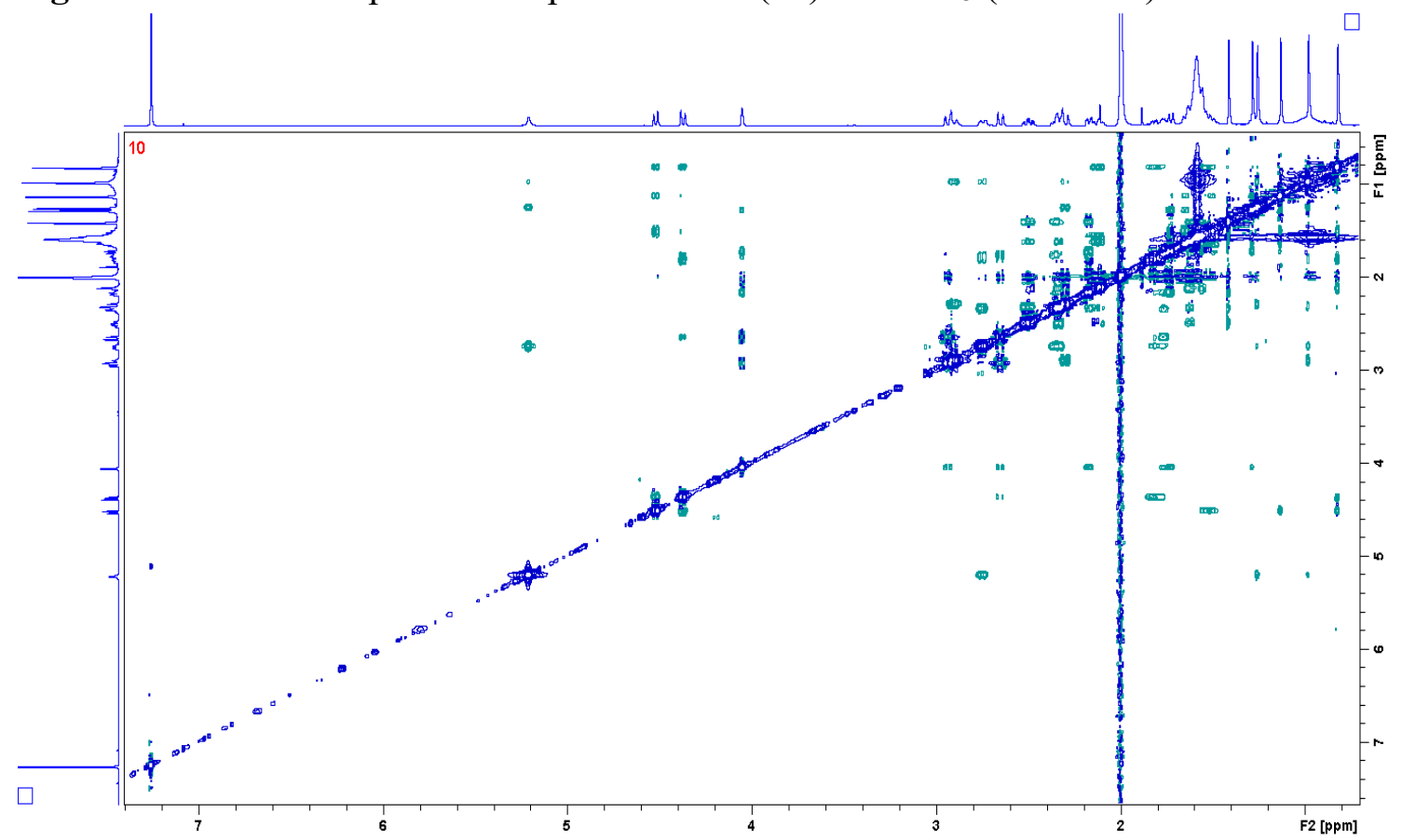


Figure S46. Expansion of the NOESY spectrum of picraviane $\mathrm{K}(10)$ in $\mathrm{CDCl}_{3}(600 \mathrm{MHz})$.

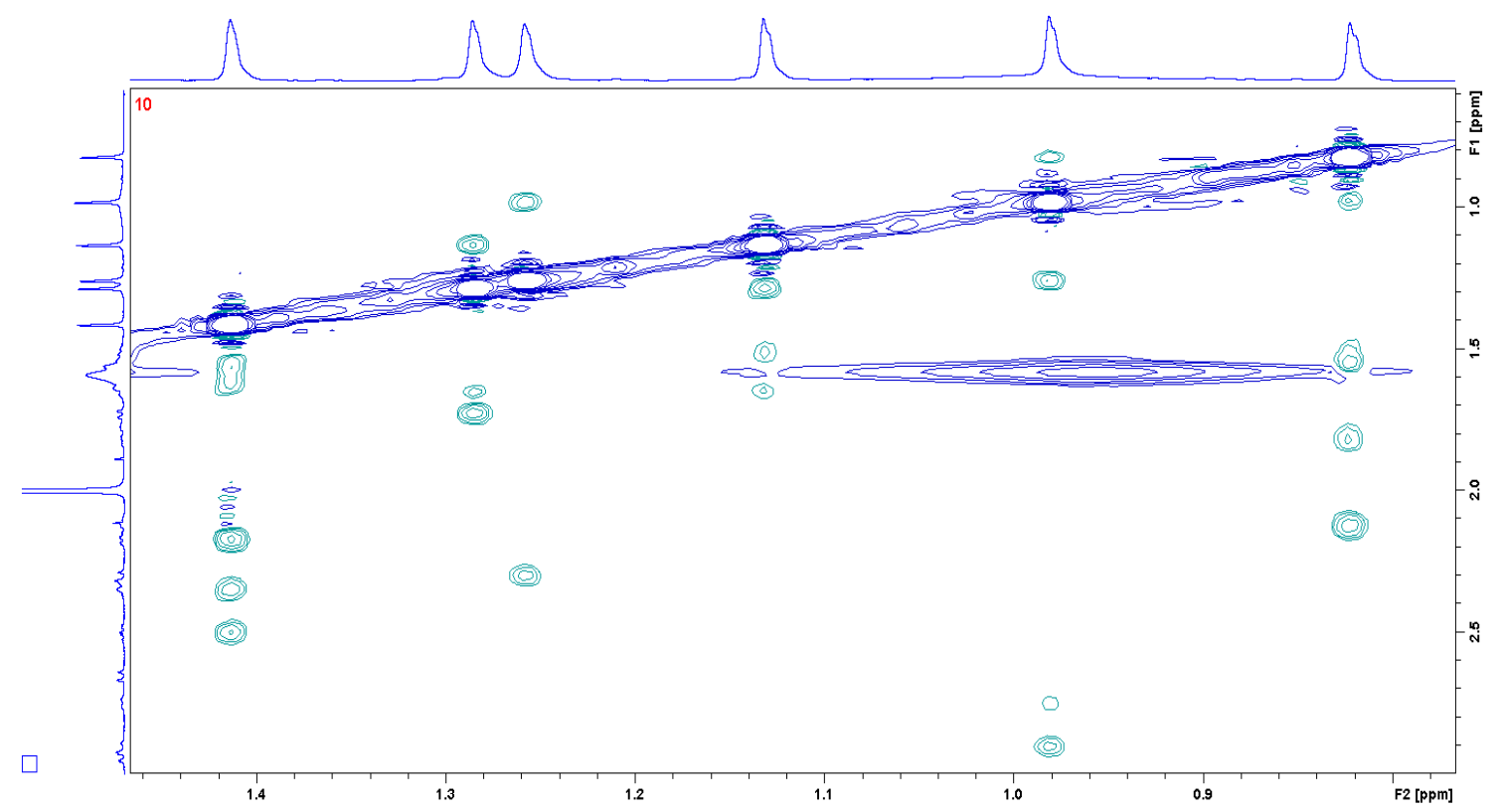


Figure S47. Structures of the two lowest-energy conformers of $(1 S, 5 R, 8 R, 9 S, 10 R, 14 S, 19 S)-9$ at the B3LYP/PCM(MeOH)/TZVP level. The simple average of the two conformers was used for calculation of the ECD spectrum.

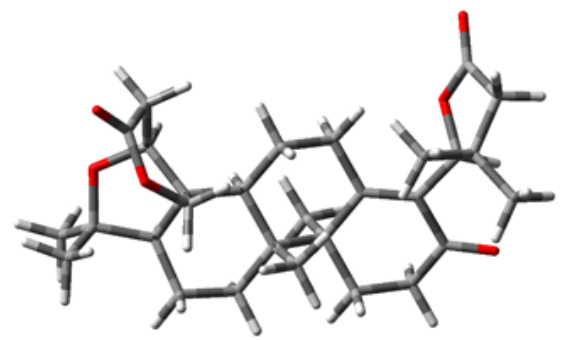

Conf. 1

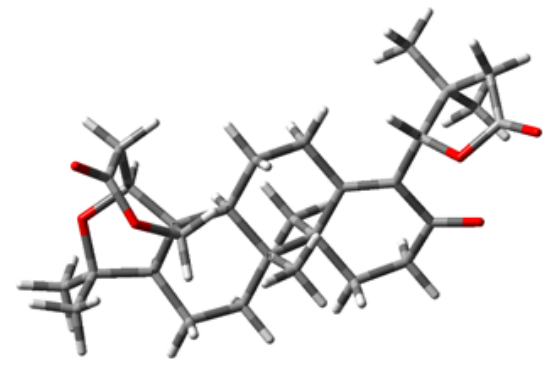

Conf. 2

Figure S48. Comparison of the experimental and calculated UV and ECD spectra of $(1 S, 5 R, 8 R, 9 S, 10 R, 14 S, 19 S)-9$ (blue trace) and its enantiomer (red trace). Experimental spectra were acquired in methanol, and UV and ECD spectra were calculated at the [CAM$\mathrm{B} 3 \mathrm{LYP} / \mathrm{PCM}(\mathrm{MeOH}) / \mathrm{TZVP}]$ level for the simple average of the two lowest-energy conformers identified.

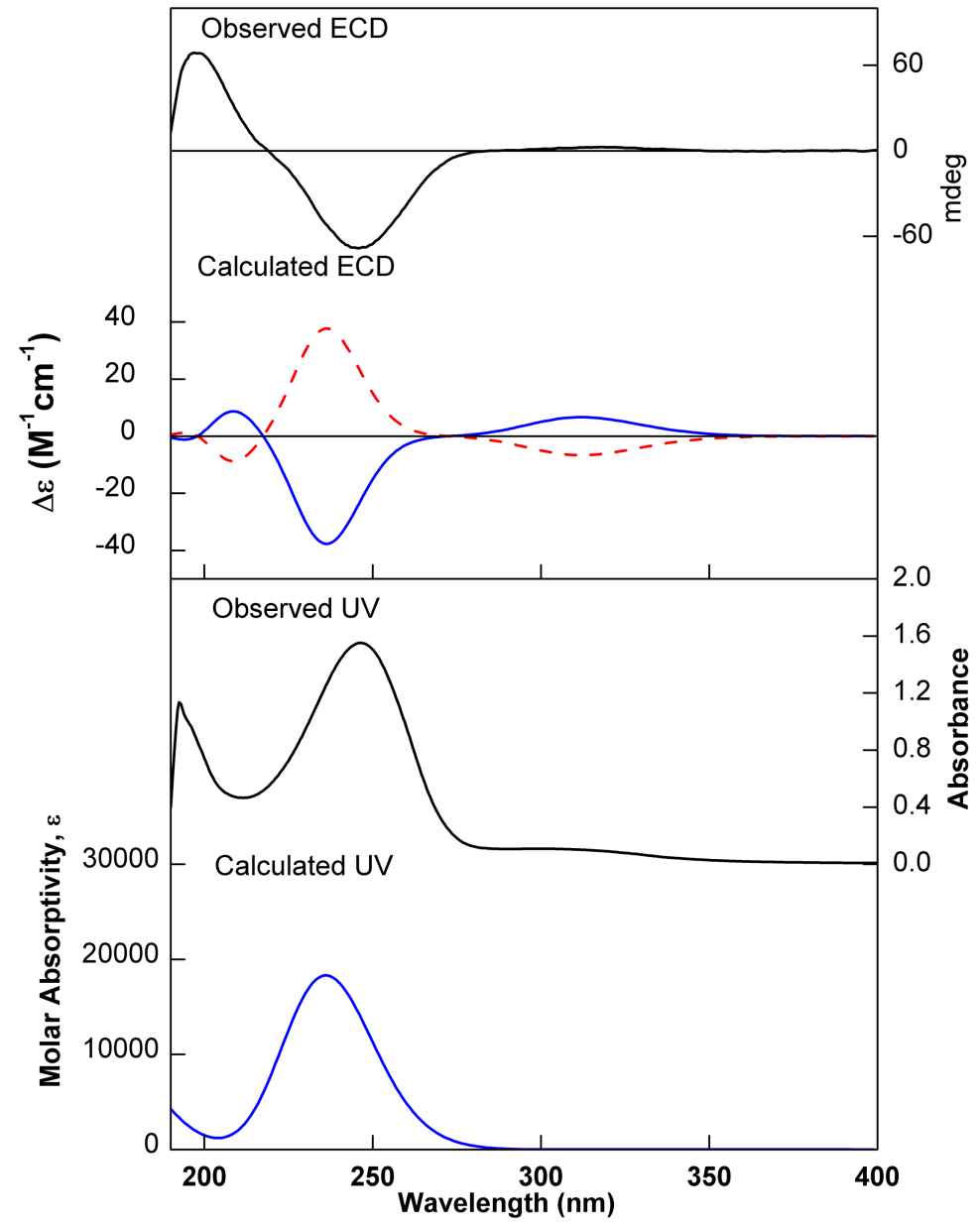


Figure S49. Structures of the two lowest-energy conformers of $(1 S, 5 R, 8 R, 9 S, 10 R, 14 S, 19 R)$ 10 at the B3LYP/PCM(MeOH)/TZVP level. The simple average of the two conformers was used for calculation of the ECD spectrum.

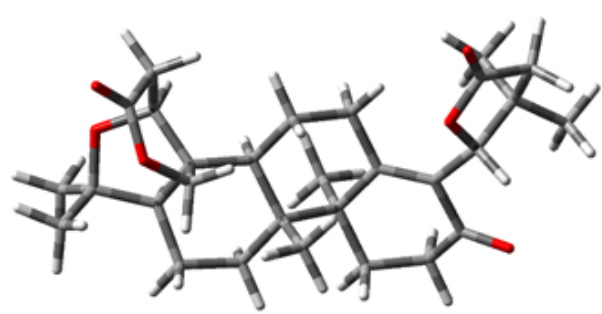

Conf. 1

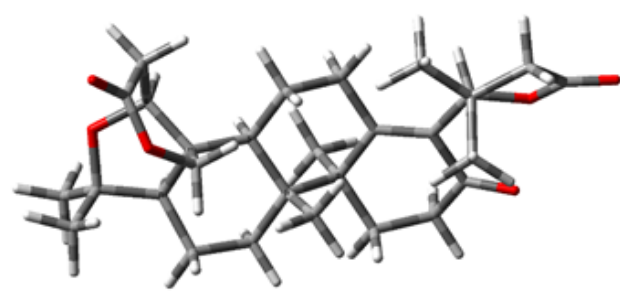

Conf. 2

Figure S50. Comparison of the experimental and calculated UV and ECD spectra of $(1 S, 5 R, 8 R, 9 S, 10 R, 14 S, 19 R)-\mathbf{1 0}$ (blue trace) and its enantiomer (red trace). Experimental spectra were acquired in methanol, and UV and ECD spectra were calculated at the [CAM$\mathrm{B} 3 \mathrm{LYP} / \mathrm{PCM}(\mathrm{MeOH}) / \mathrm{TZVP}]$ level for the simple average of the two lowest-energy conformers identified.

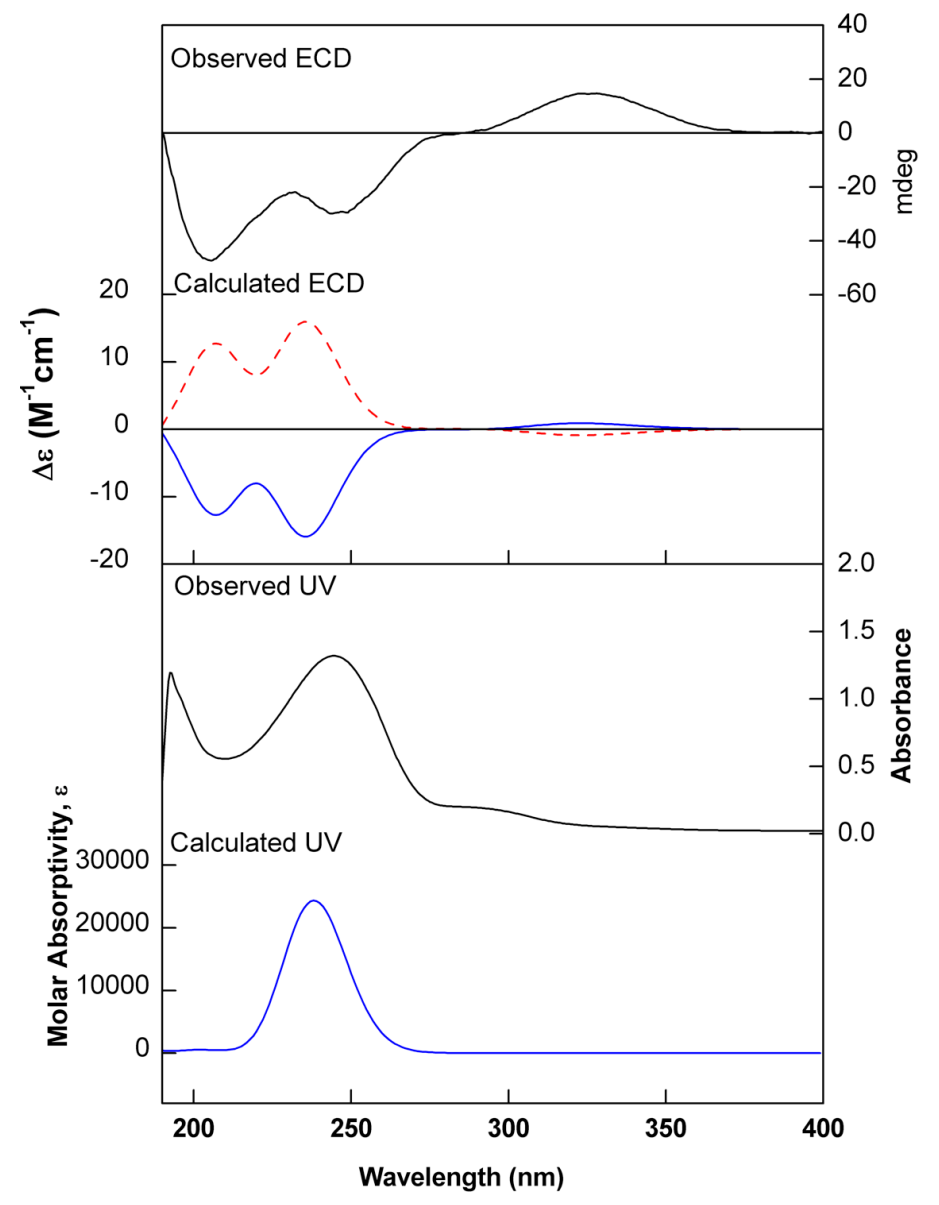


Figure S51. ${ }^{1} \mathrm{H}$ NMR spectrum of picraviane D (2) in $\mathrm{CDCl}_{3}(600 \mathrm{MHz})$.

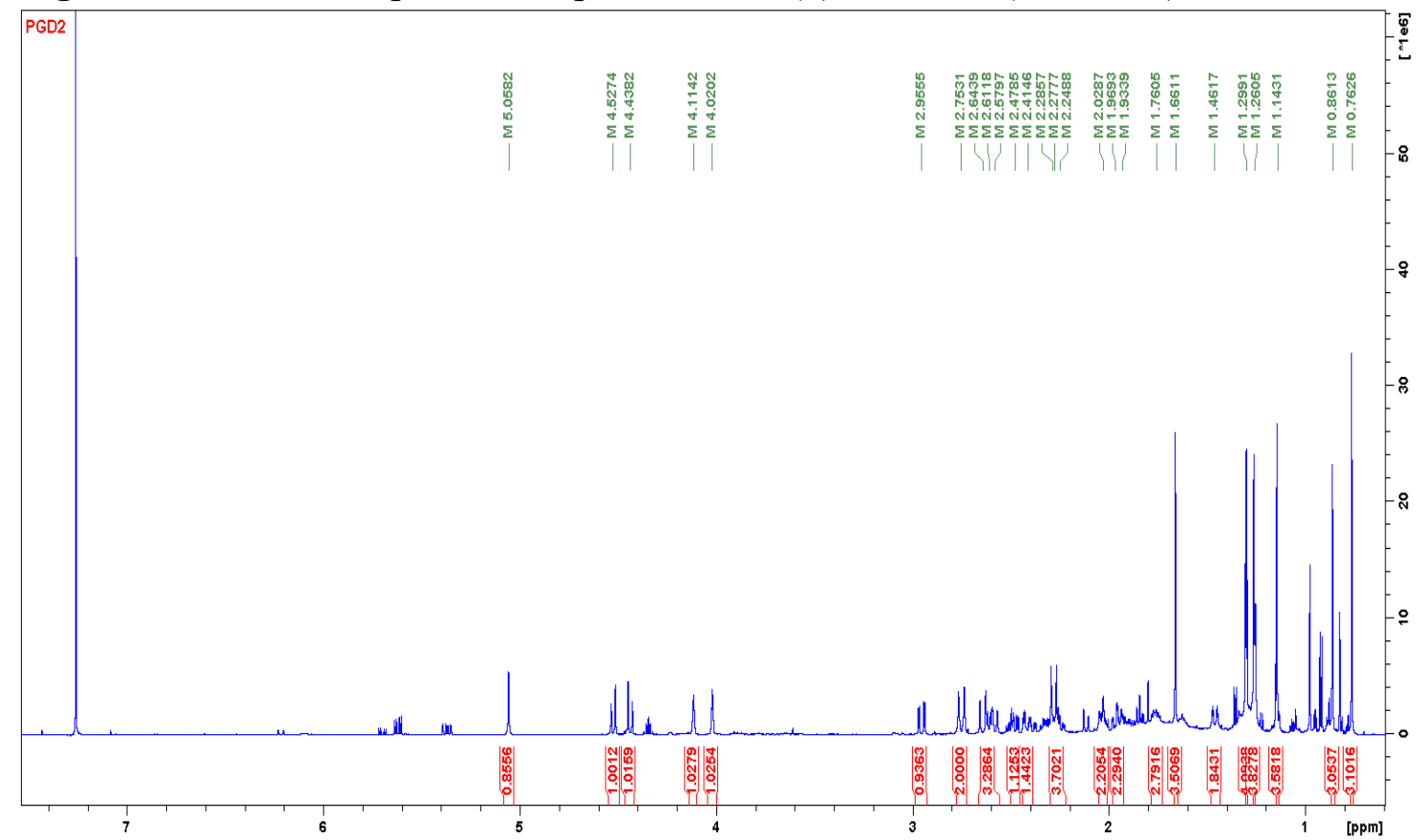

Figure S52. Expansion of the ${ }^{1} \mathrm{H}$ NMR spectrum of picraviane D (2) in $\mathrm{CDCl}_{3}(600 \mathrm{MHz})$.

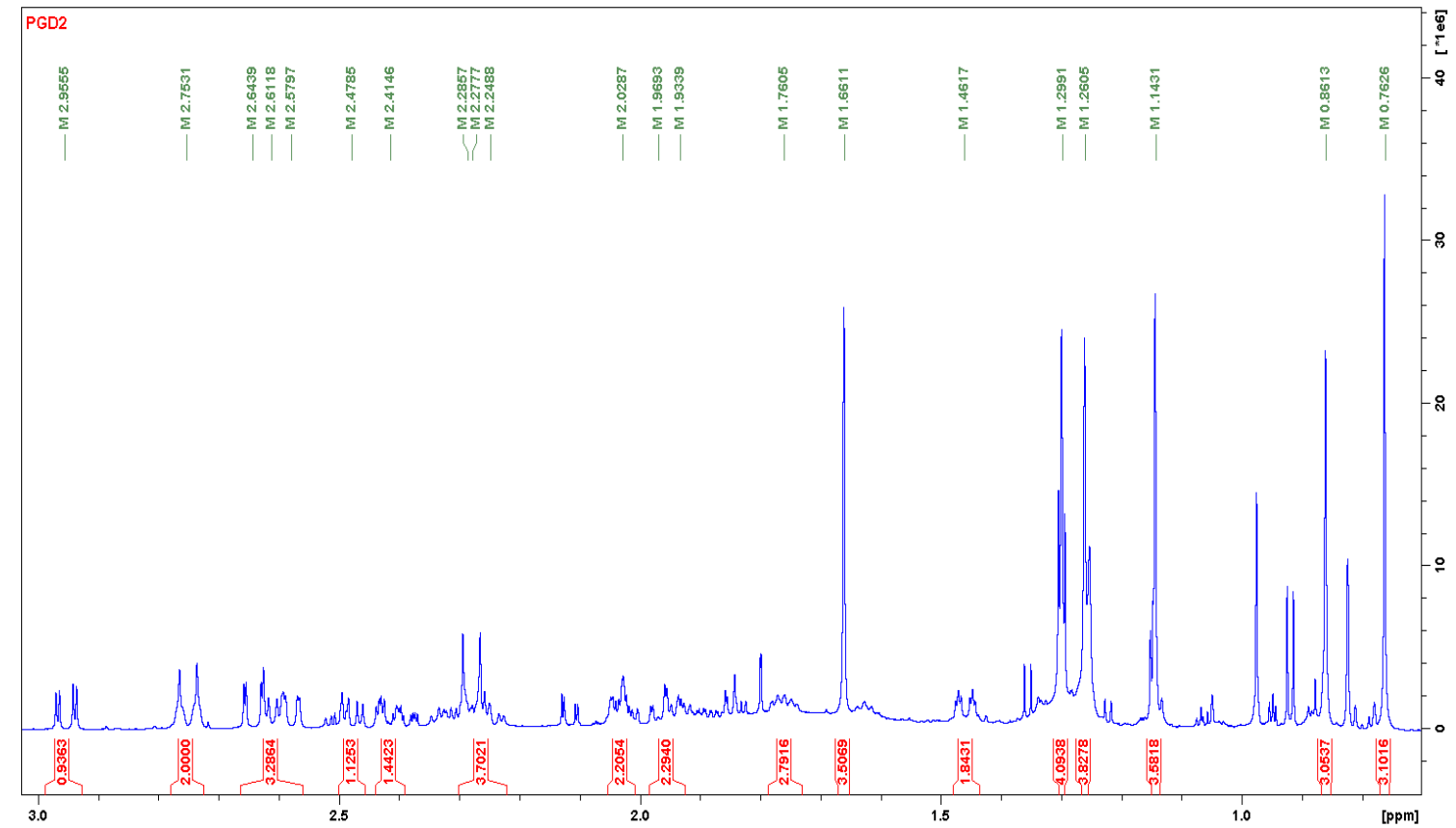


Figure S53. ${ }^{13} \mathrm{C}$ NMR spectrum of picraviane D (2) in $\mathrm{CDCl}_{3}(150 \mathrm{MHz})$.

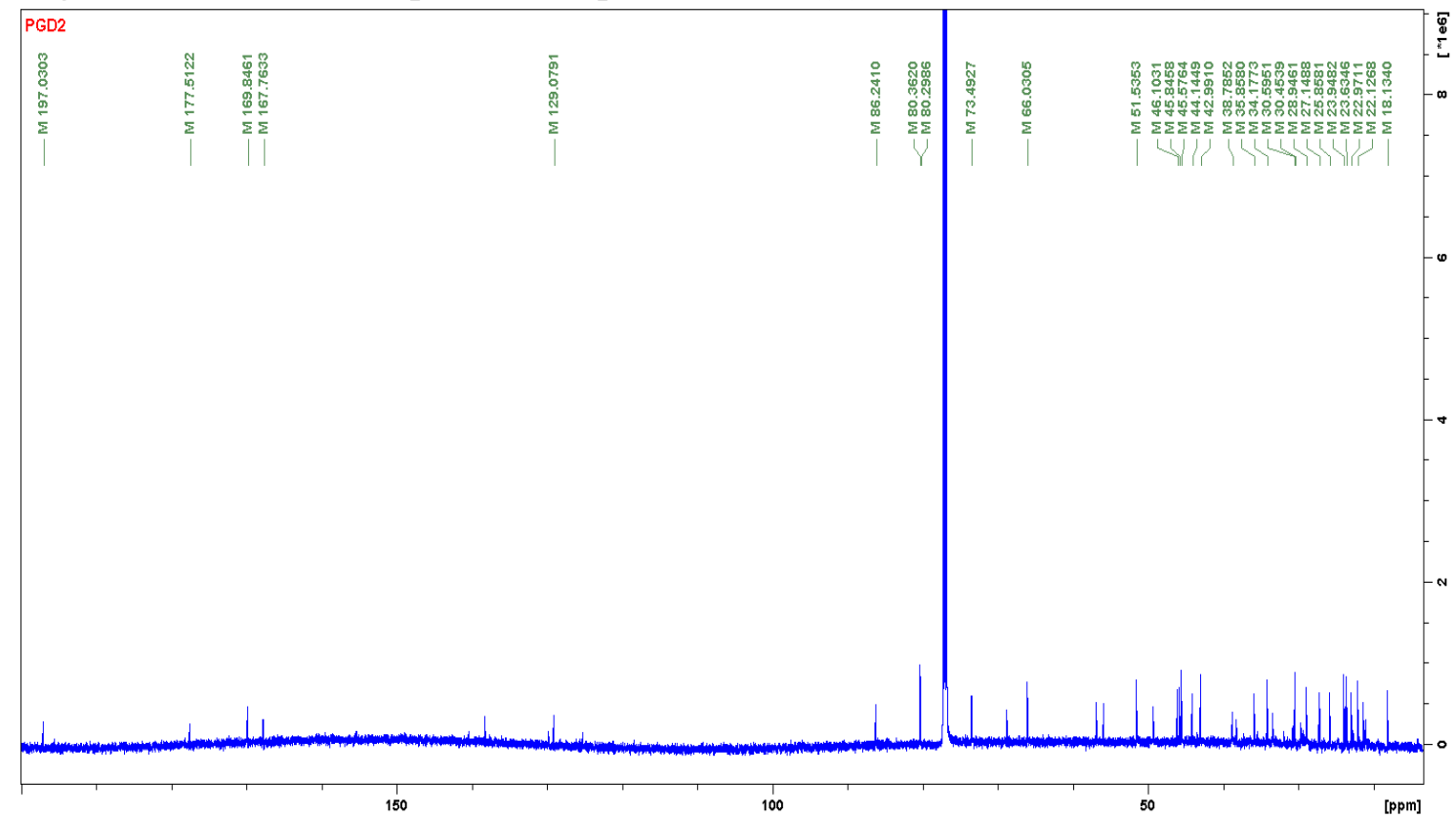

Figure S54. HSQC spectrum of picraviane D (2) in $\mathrm{CDCl}_{3}(600 \mathrm{MHz})$.

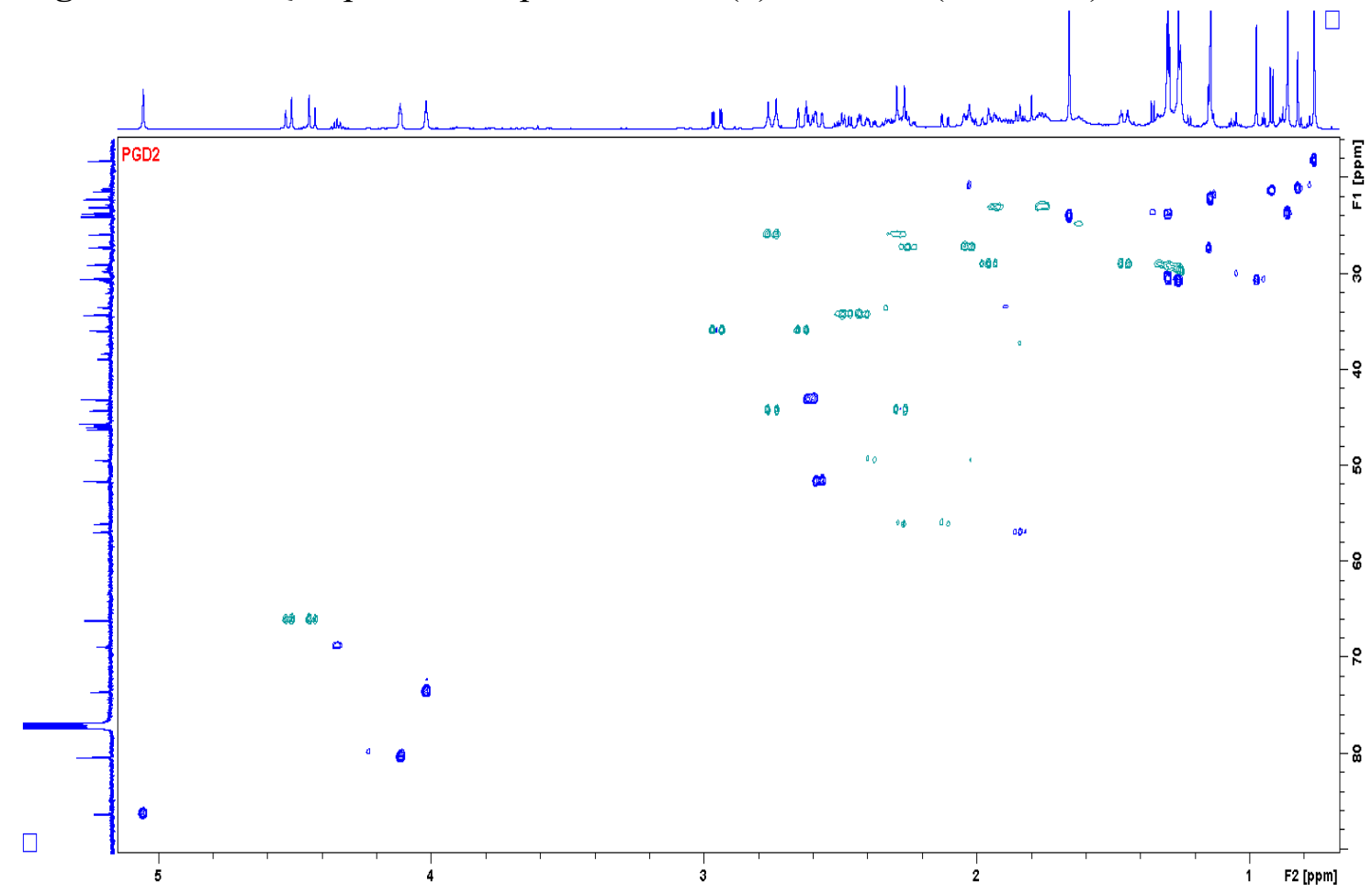


Figure S55. HMBC spectrum of picraviane D (2) in $\mathrm{CDCl}_{3}(600 \mathrm{MHz})$.

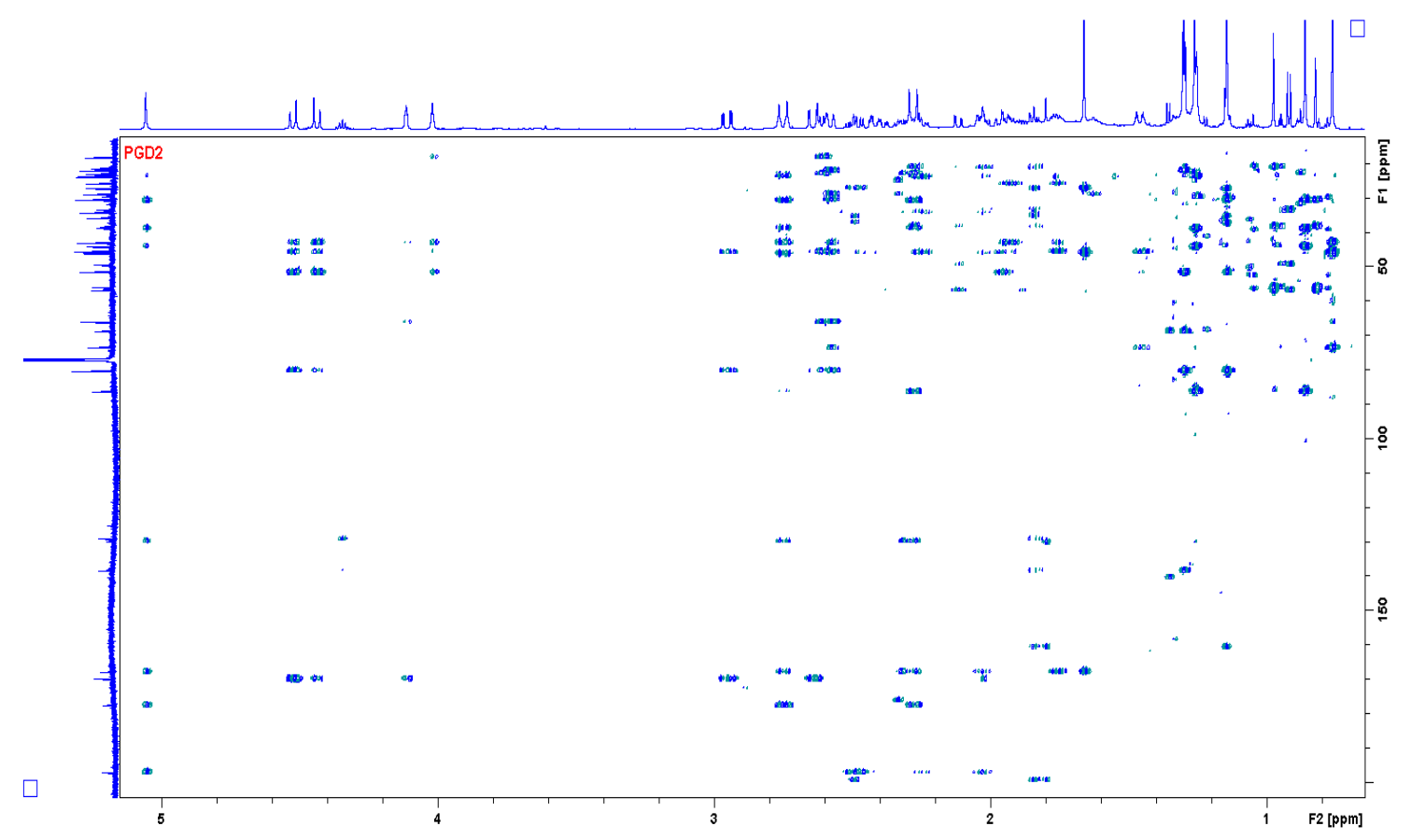

Figure S56. COSY spectrum of picraviane D (2) in $\mathrm{CDCl}_{3}(600 \mathrm{MHz})$.

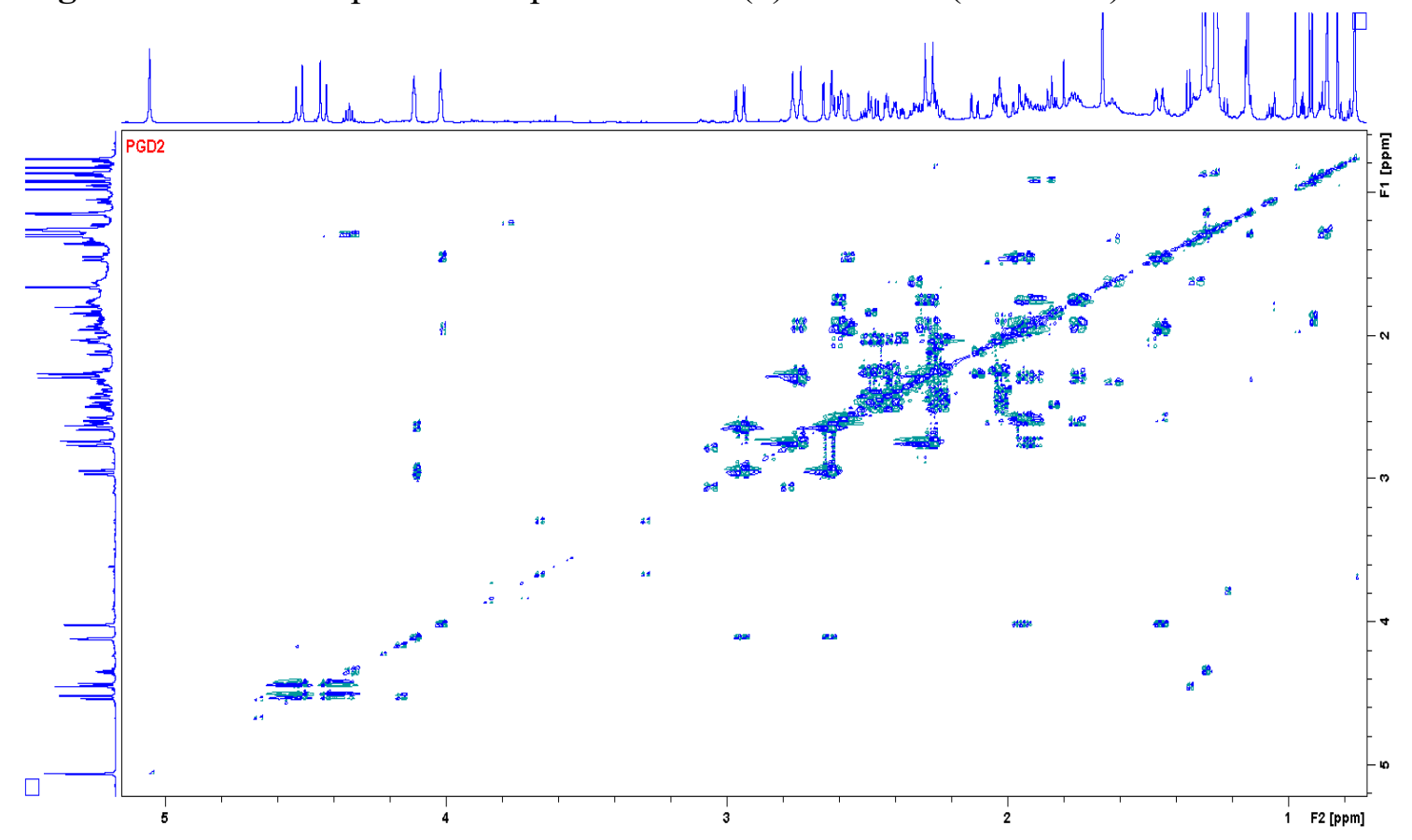


Figure S57. NOESY spectrum of picraviane D (2) in $\mathrm{CDCl}_{3}(600 \mathrm{MHz})$.

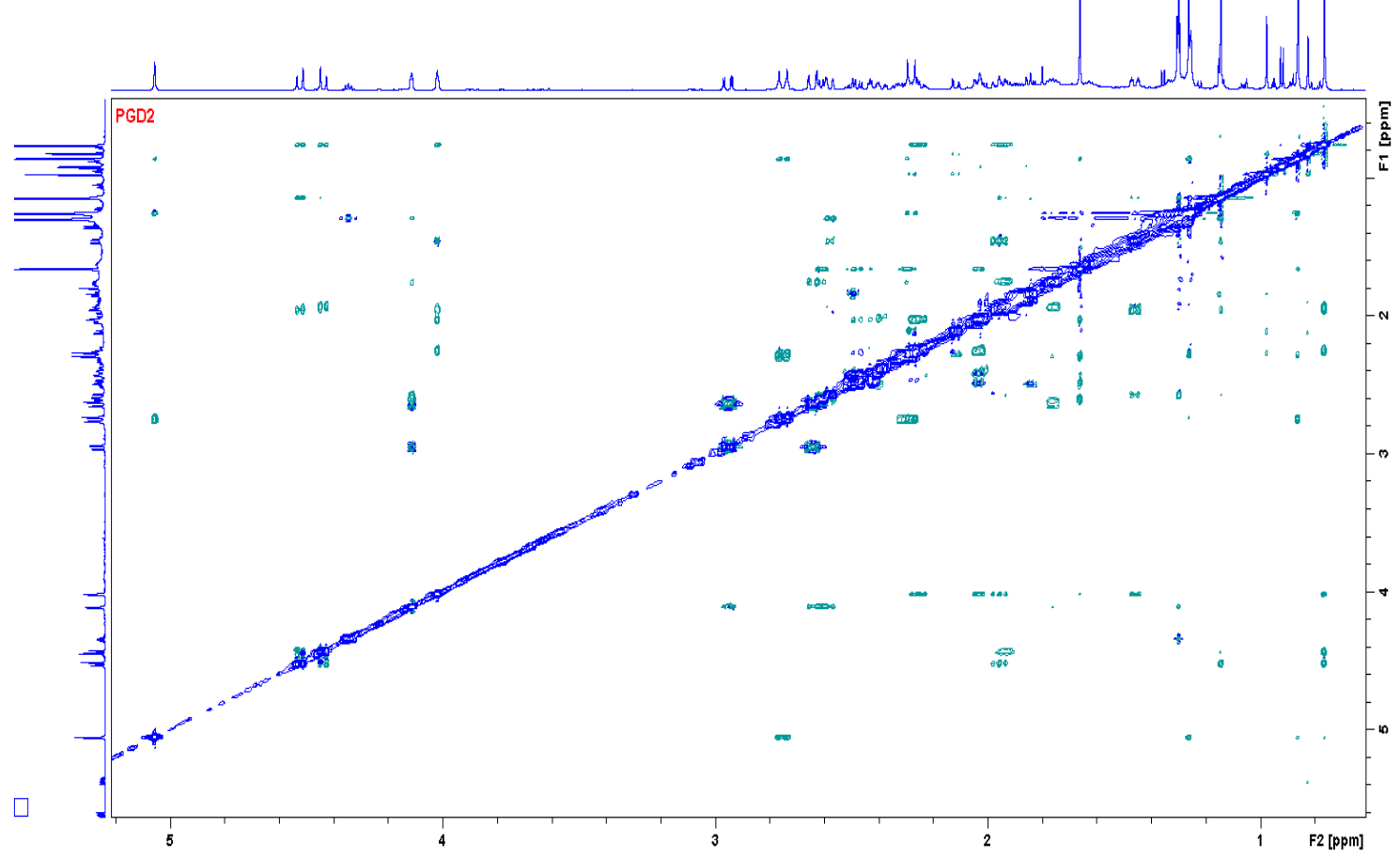

Figure S58. Expansion of the NOESY spectrum of picraviane D (2) in $\mathrm{CDCl}_{3}(600 \mathrm{MHz})$.

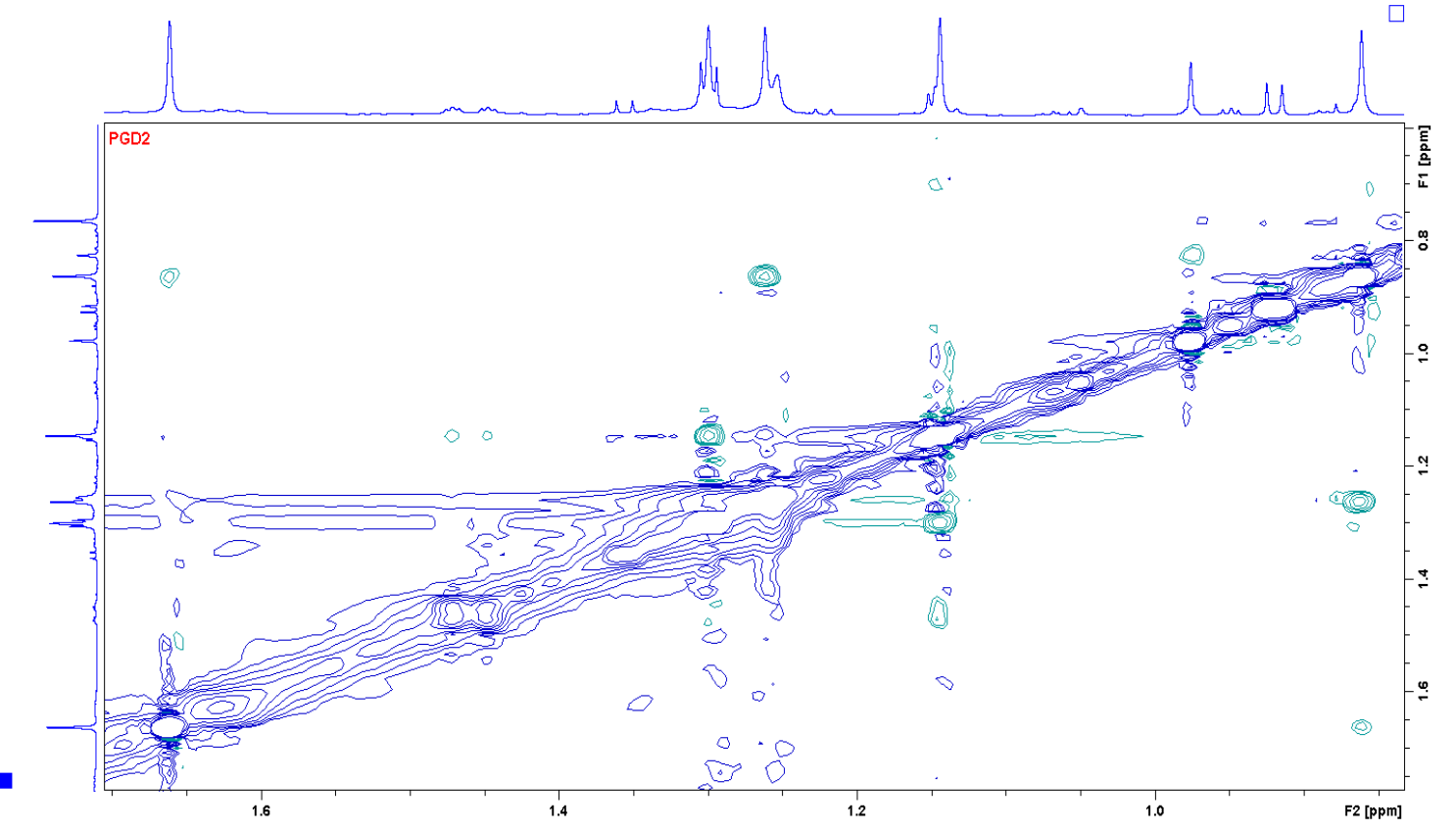

41 
Figure S59. ${ }^{1} \mathrm{H}$ NMR spectrum of picraviane $\mathrm{E}(3)$ in $\mathrm{CDCl}_{3}(600 \mathrm{MHz})$.

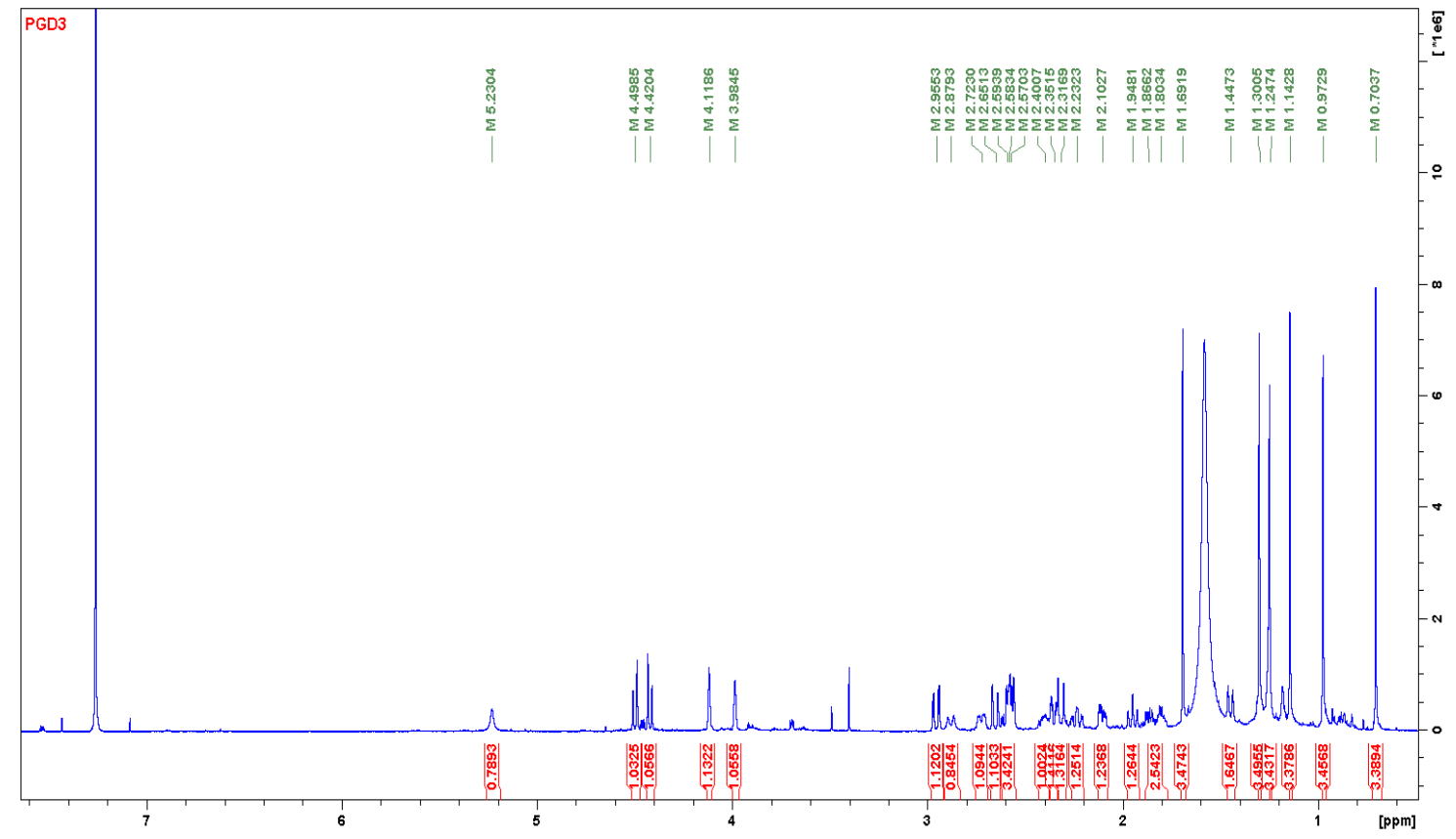

Figure S60. Expansion of the ${ }^{1} \mathrm{H}$ NMR spectrum of picraviane $\mathrm{E}(3)$ in $\mathrm{CDCl}_{3}(600 \mathrm{MHz})$.

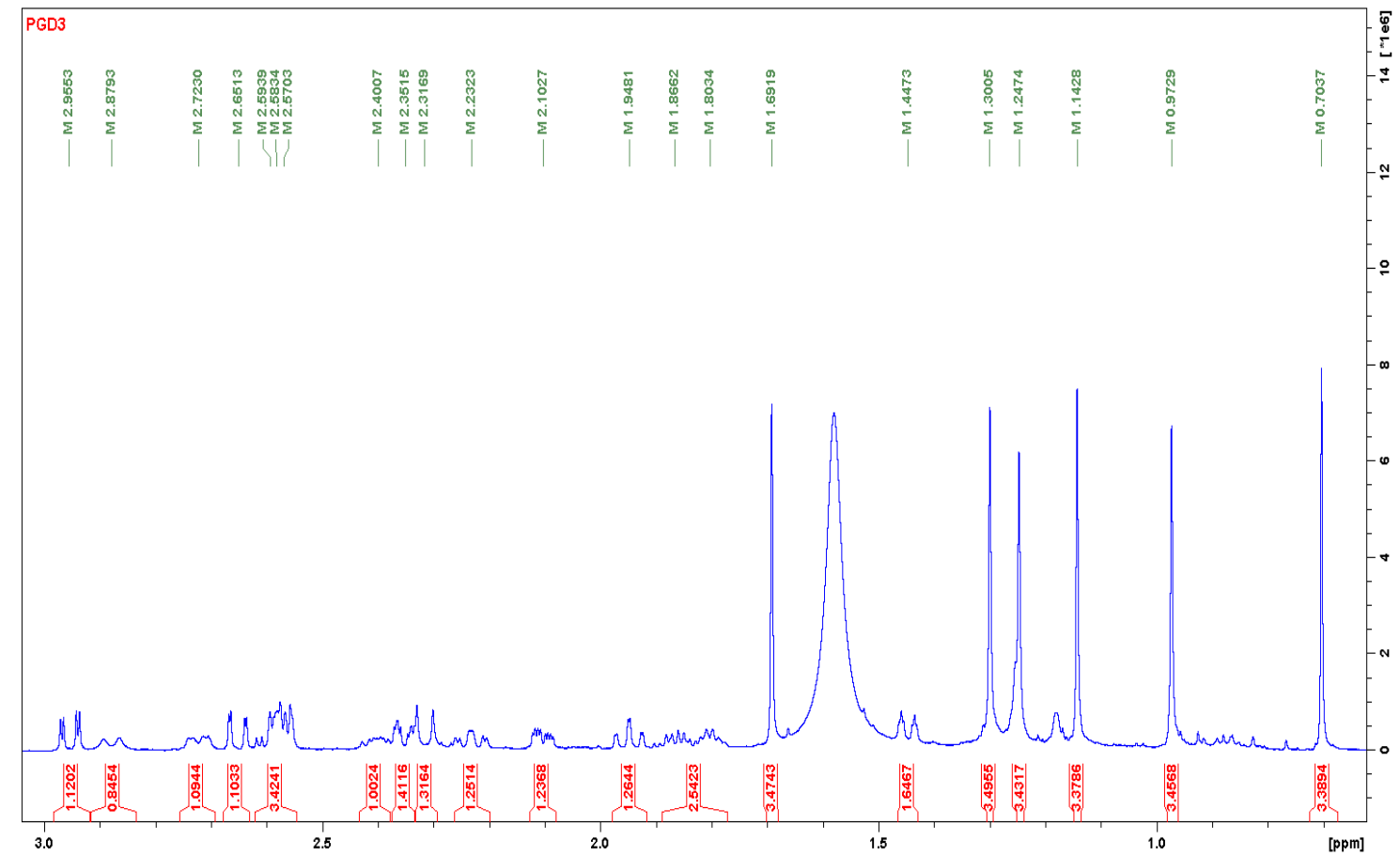


Figure S61. ${ }^{13} \mathrm{C}$ NMR spectrum of picraviane $\mathrm{E}(3)$ in $\mathrm{CDCl}_{3}(150 \mathrm{MHz})$.

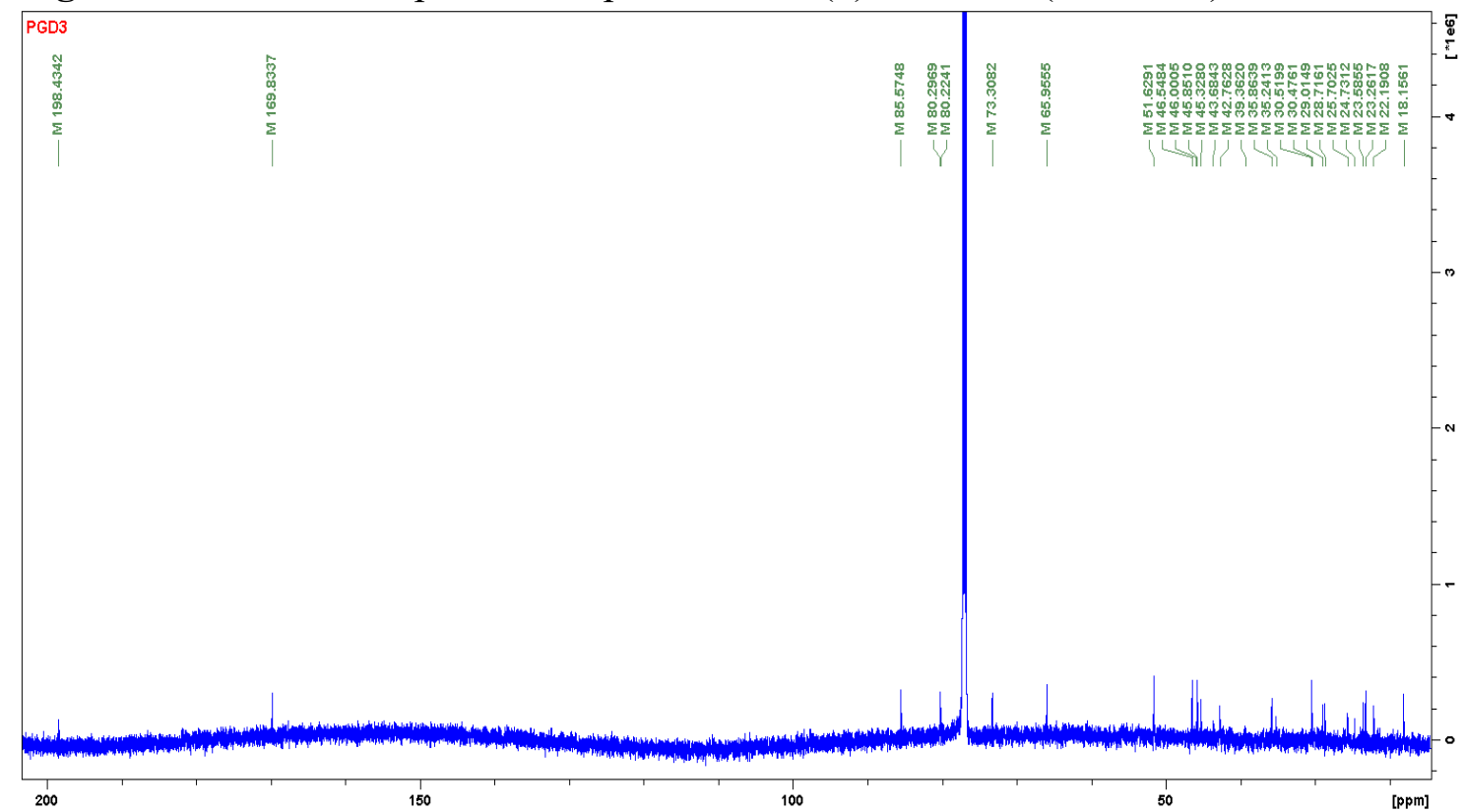

Figure S62. HSQC spectrum of picraviane $\mathrm{E}(\mathbf{3})$ in $\mathrm{CDCl}_{3}(600 \mathrm{MHz})$.

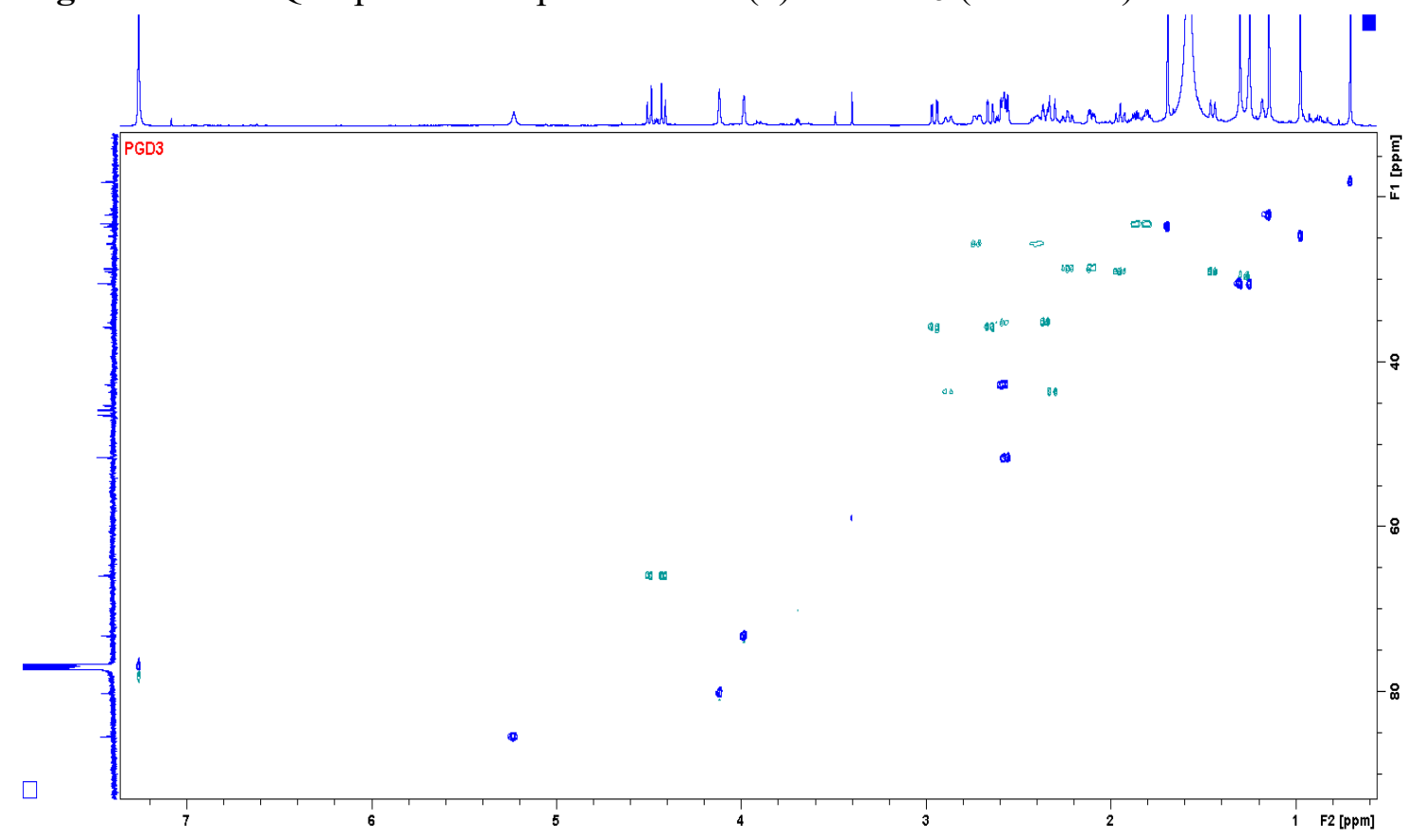


Figure S63. HMBC spectrum of picraviane $\mathrm{E}(3)$ in $\mathrm{CDCl}_{3}(600 \mathrm{MHz})$.

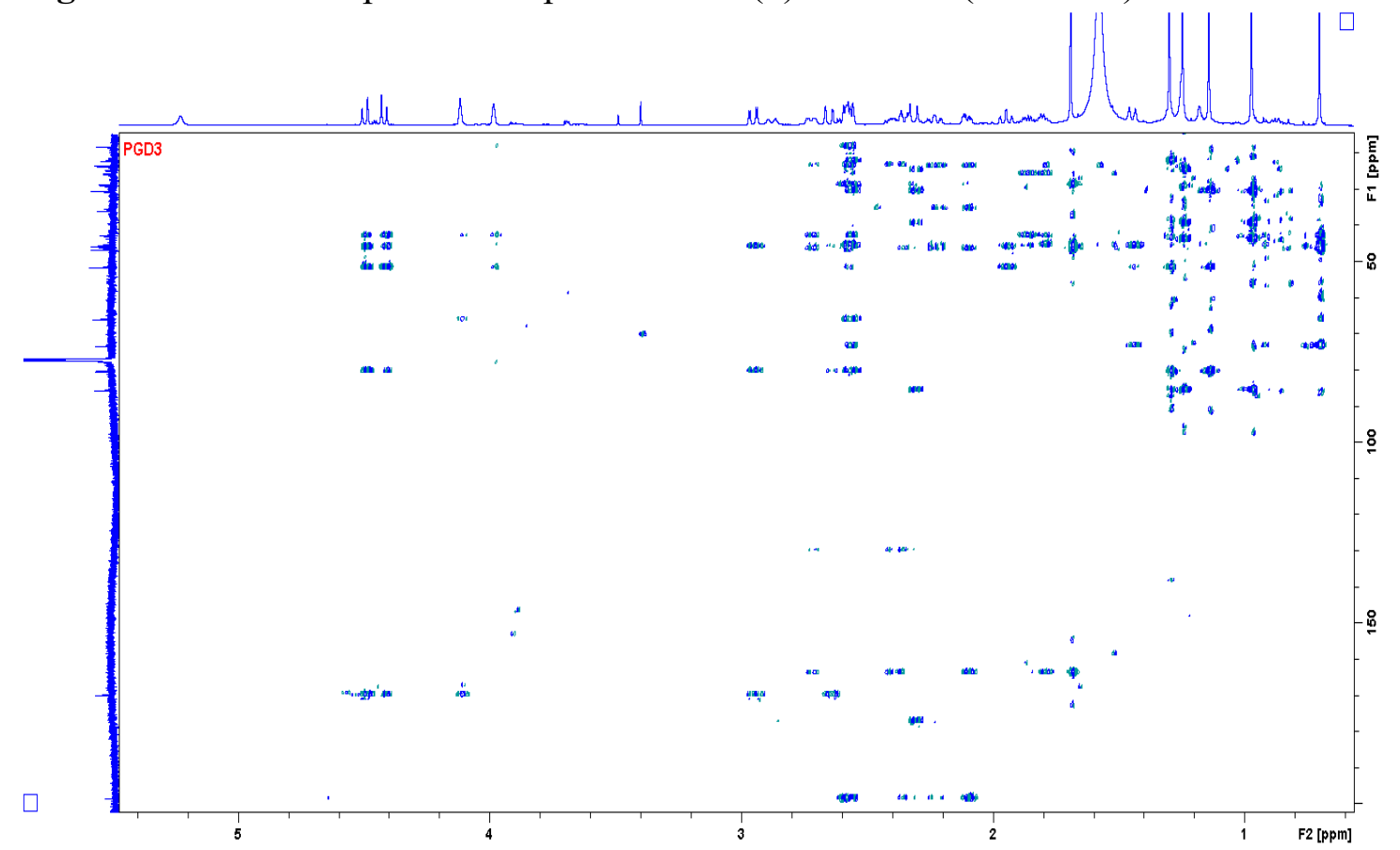

Figure S64. COSY spectrum of picraviane $\mathrm{E}(3)$ in $\mathrm{CDCl}_{3}(600 \mathrm{MHz})$.

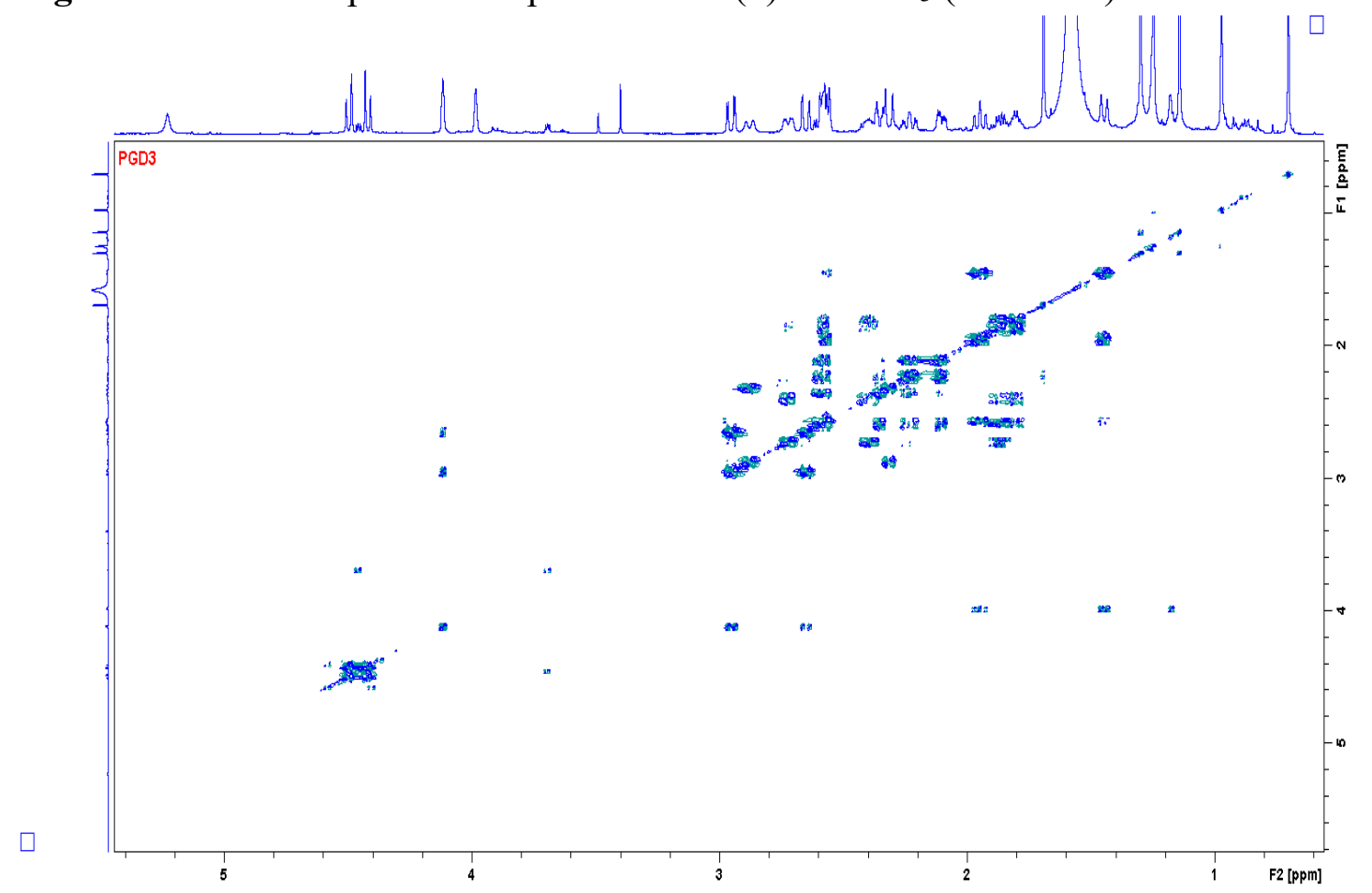


Figure S65. NOESY spectrum of picraviane E (3) in $\mathrm{CDCl}_{3}(600 \mathrm{MHz})$.

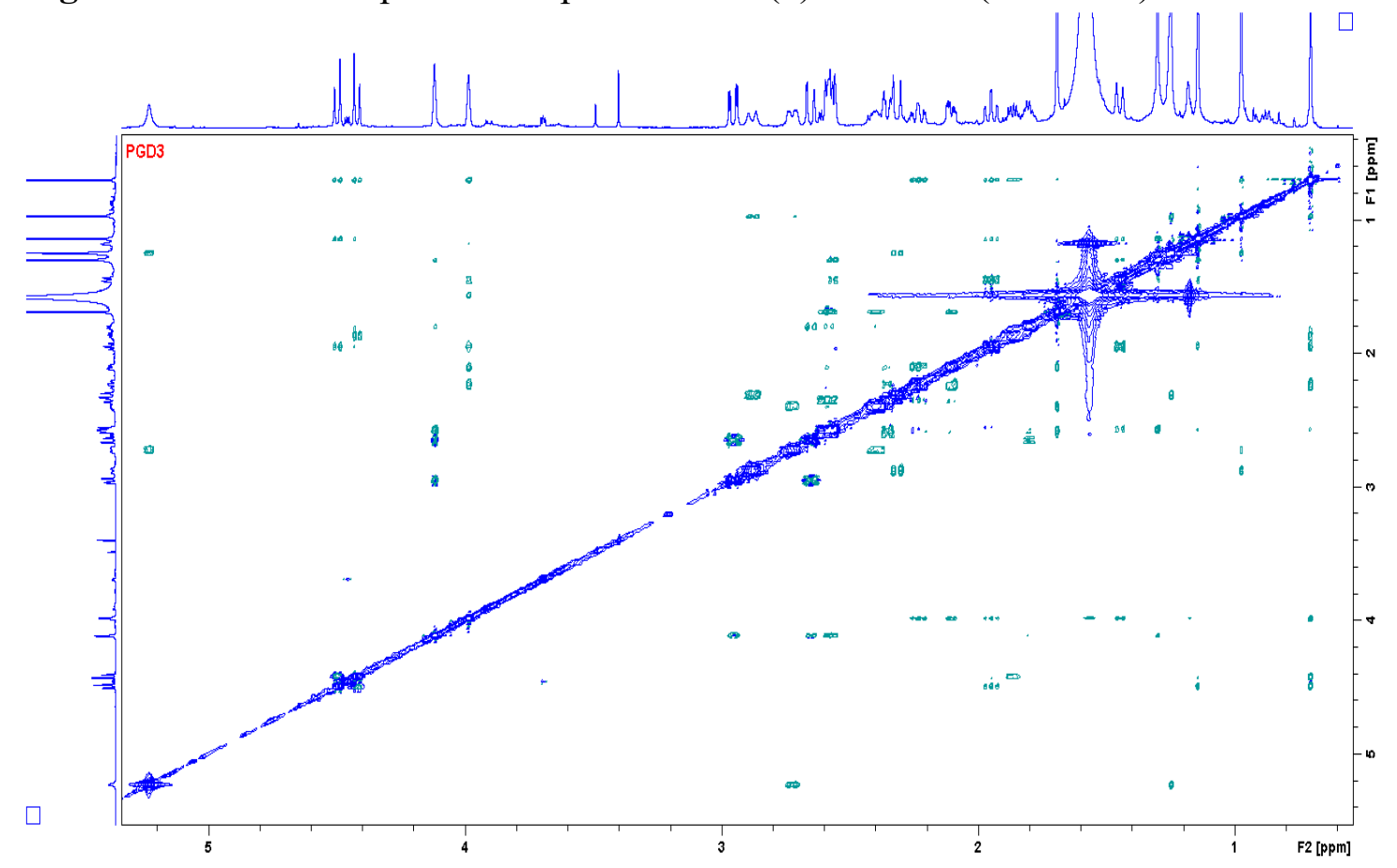

Figure S66. Expansion of the NOESY spectrum of picraviane E (3) in $\mathrm{CDCl}_{3}(600 \mathrm{MHz})$.

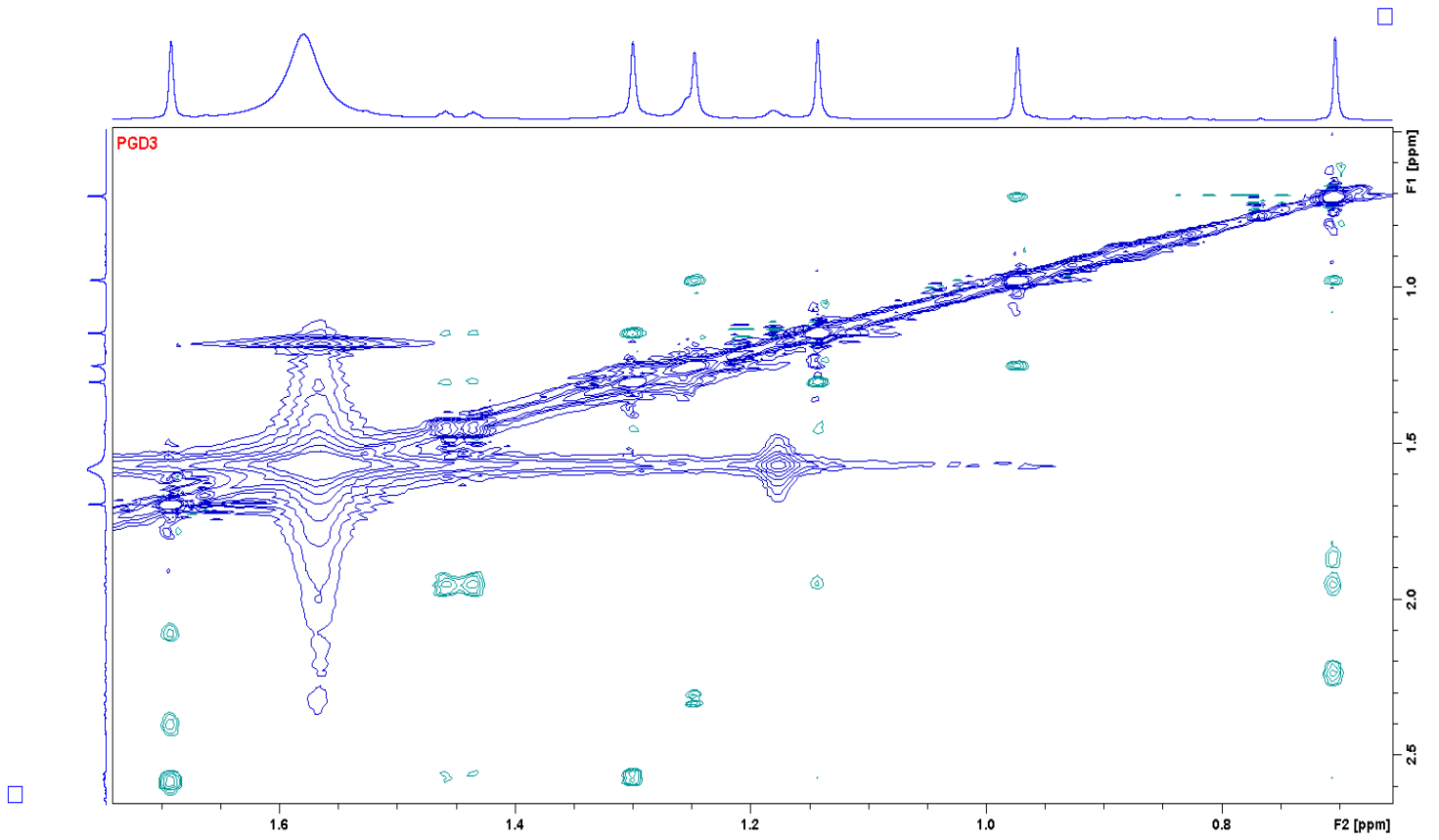


Figure S67. ${ }^{1} \mathrm{H}$ NMR spectrum of picraviane $\mathrm{R}(18)$ in $\mathrm{CDCl}_{3}(600 \mathrm{MHz})$.

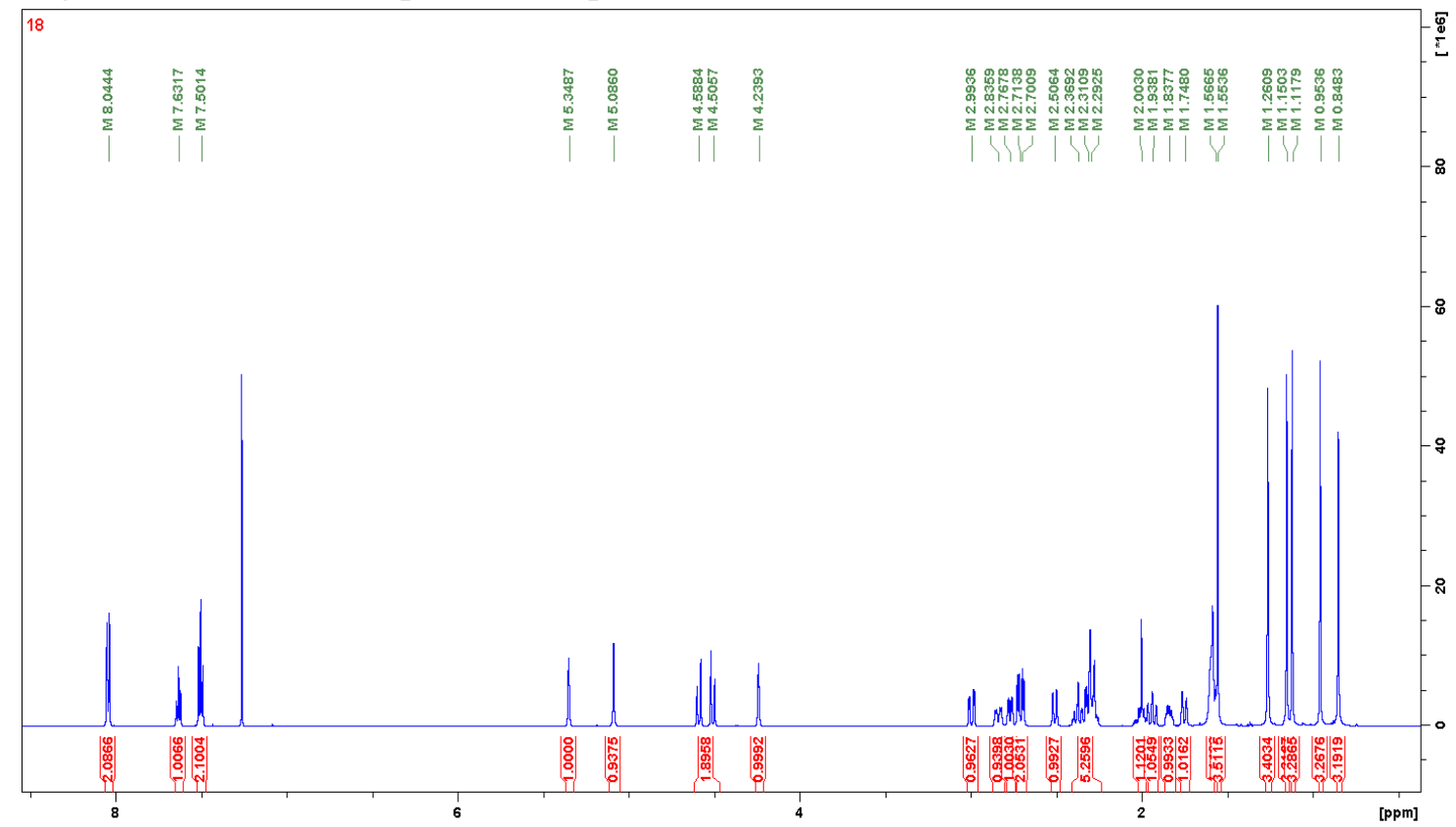

Figure S68. Expansion of the ${ }^{1} \mathrm{H}$ NMR spectrum of picraviane R (18) in $\mathrm{CDCl}_{3}(600 \mathrm{MHz})$.

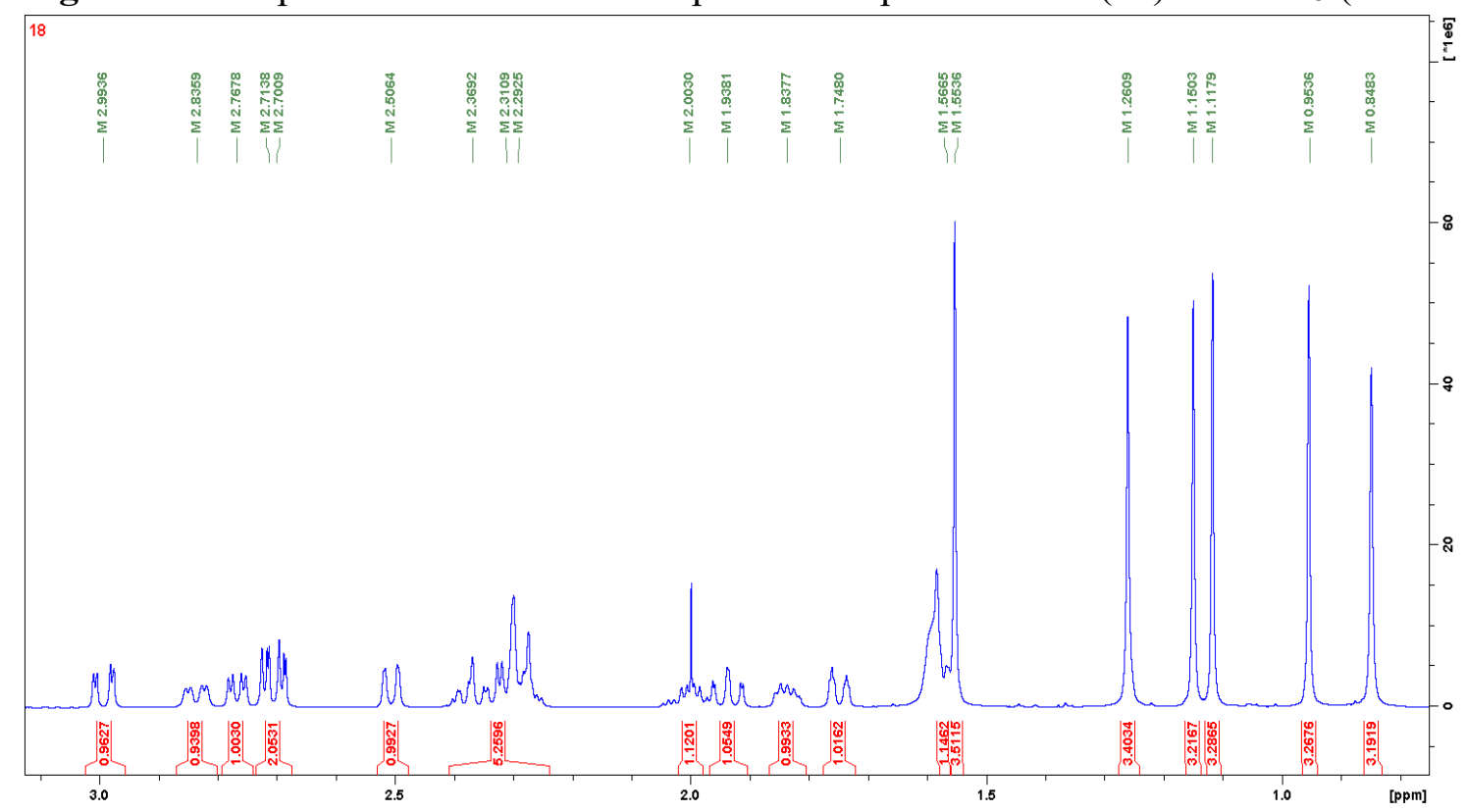


Figure S69. ${ }^{13} \mathrm{C}$ NMR spectrum of picraviane R (18) in $\mathrm{CDCl}_{3}(150 \mathrm{MHz})$.

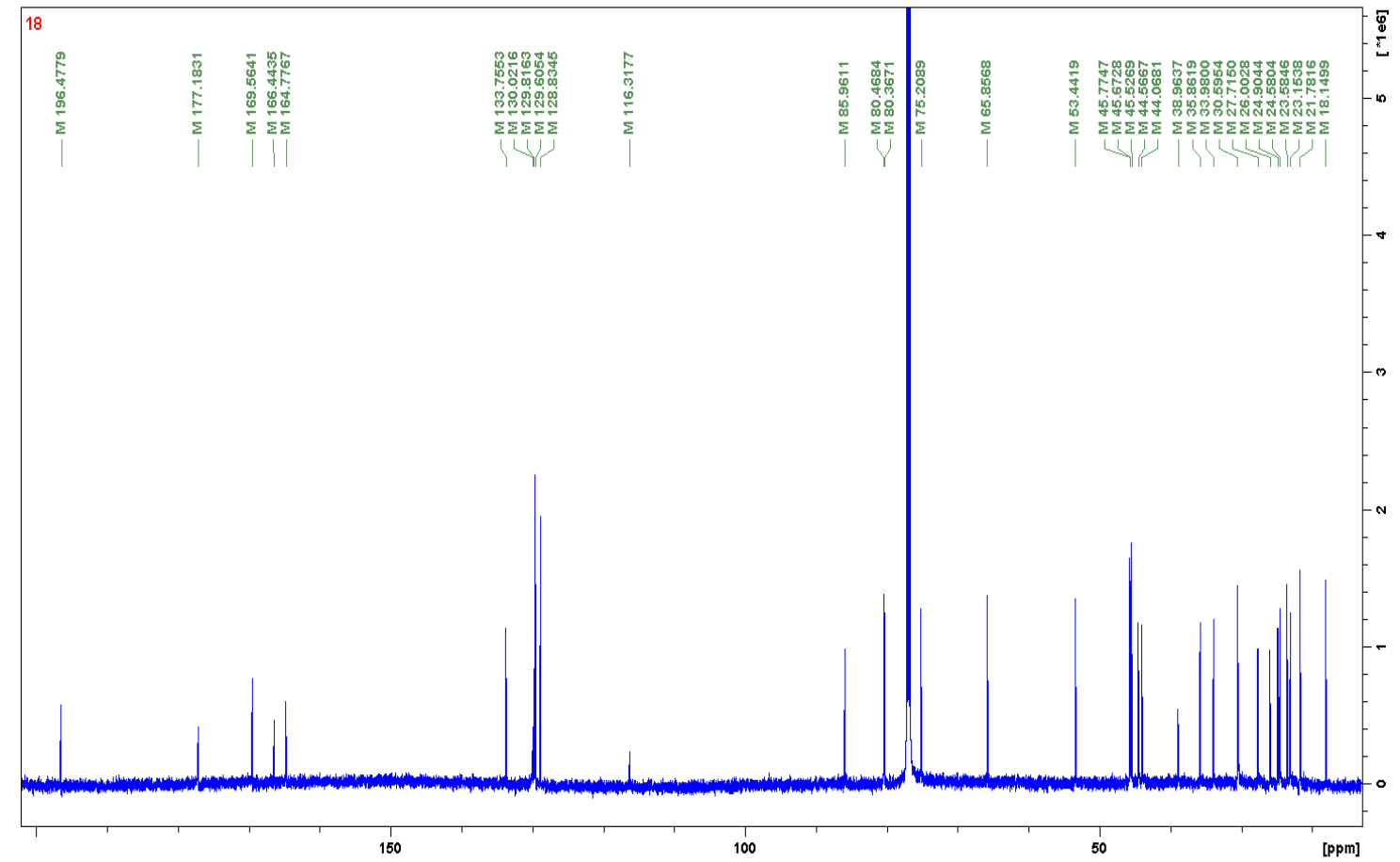

Figure S70. HSQC spectrum of picraviane R (18) in $\mathrm{CDCl}_{3}(600 \mathrm{MHz})$.

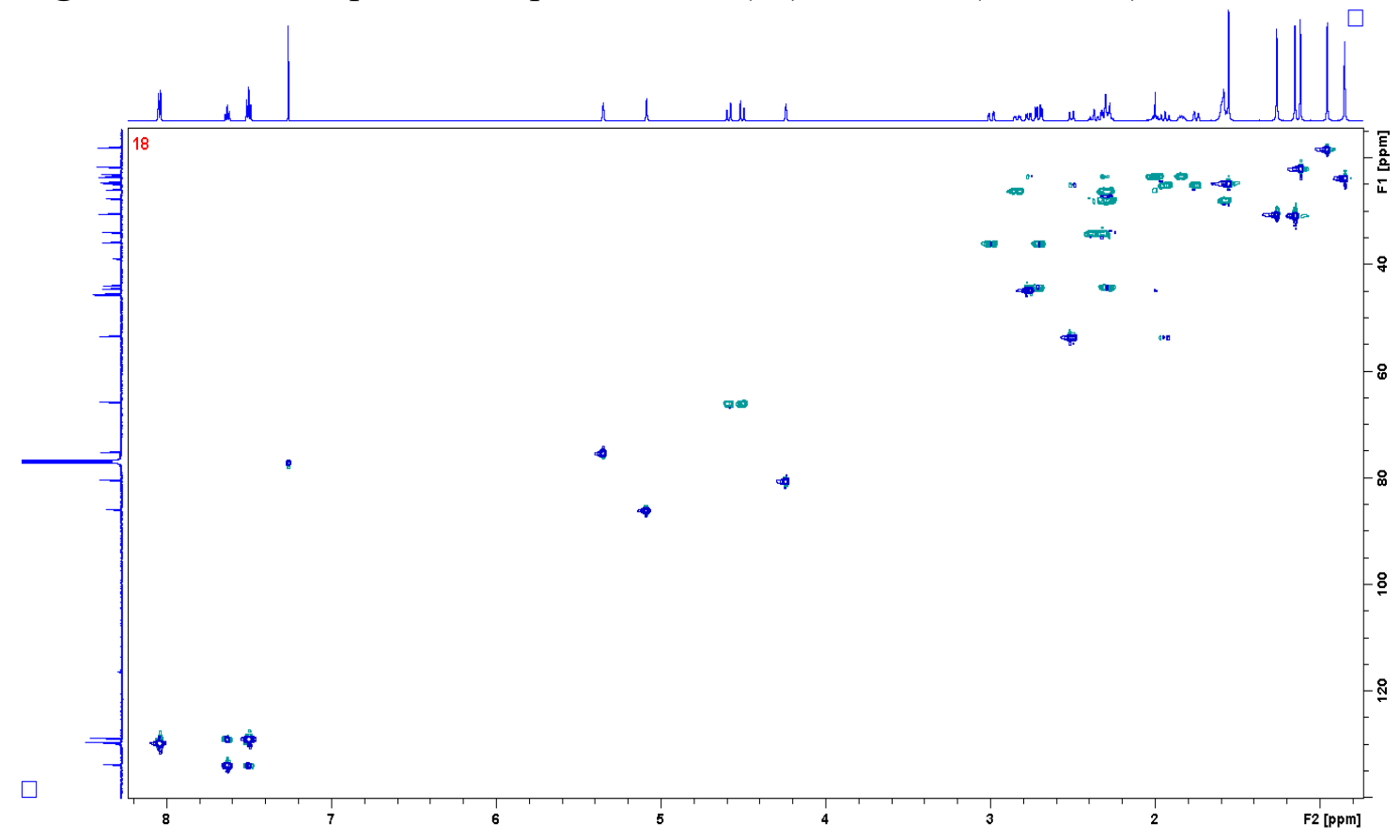


Figure S71. HMBC spectrum of picraviane R (18) in $\mathrm{CDCl}_{3}(600 \mathrm{MHz})$.

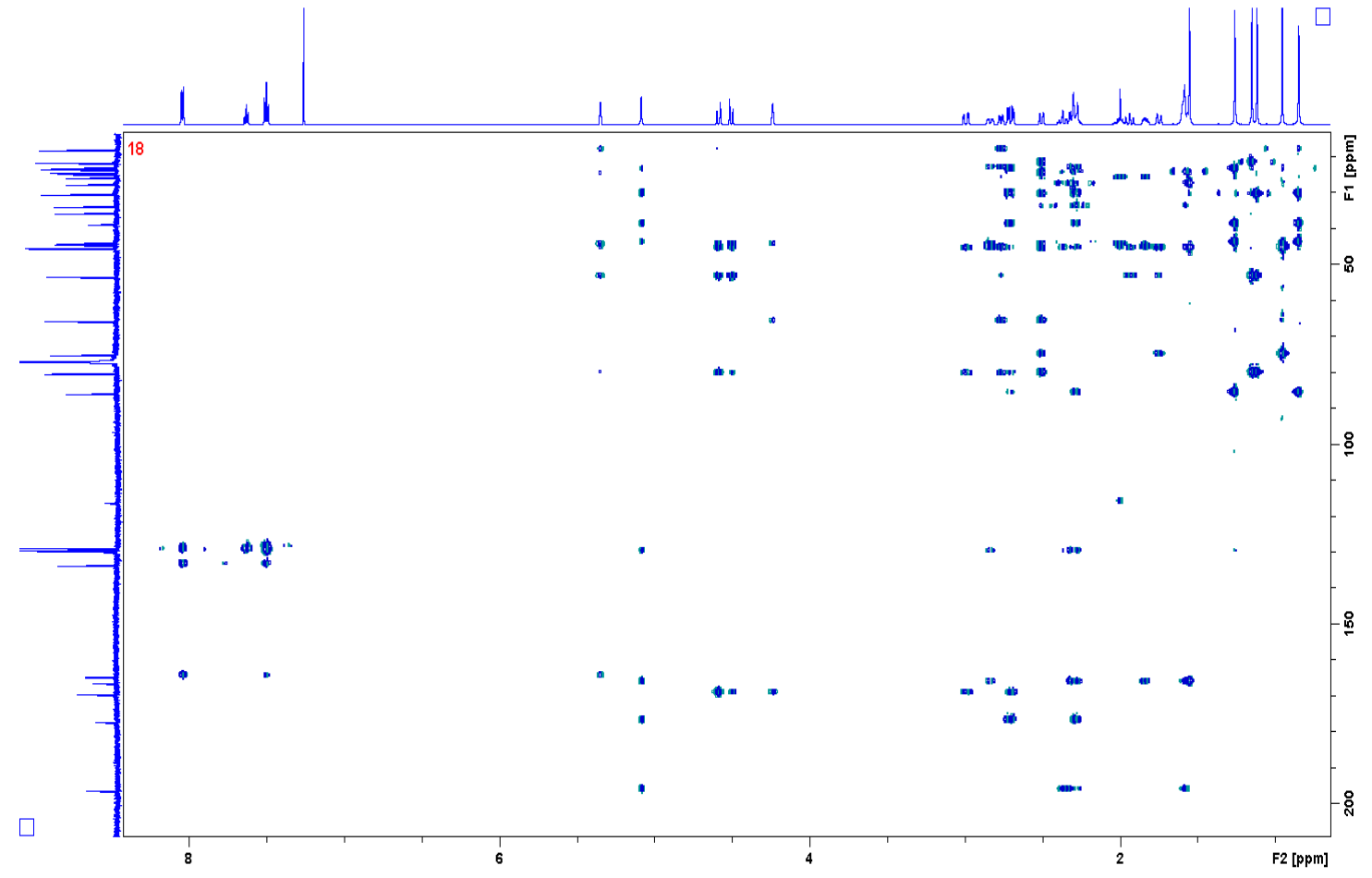

Figure S72. COSY spectrum of picraviane R (18) in $\mathrm{CDCl}_{3}(600 \mathrm{MHz})$.

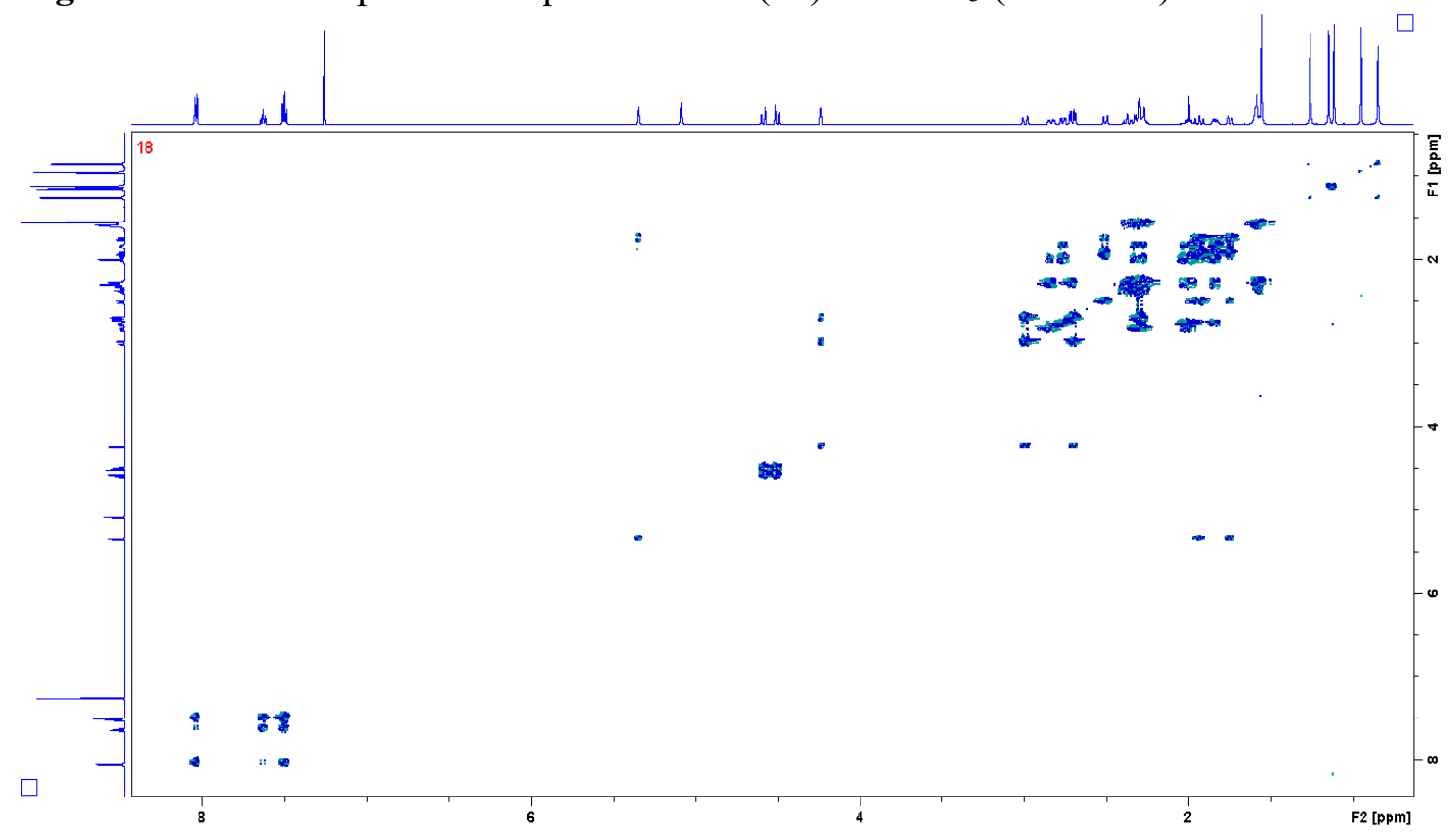


Figure S73. NOESY spectrum of picraviane R (18) in $\mathrm{CDCl}_{3}(600 \mathrm{MHz})$.

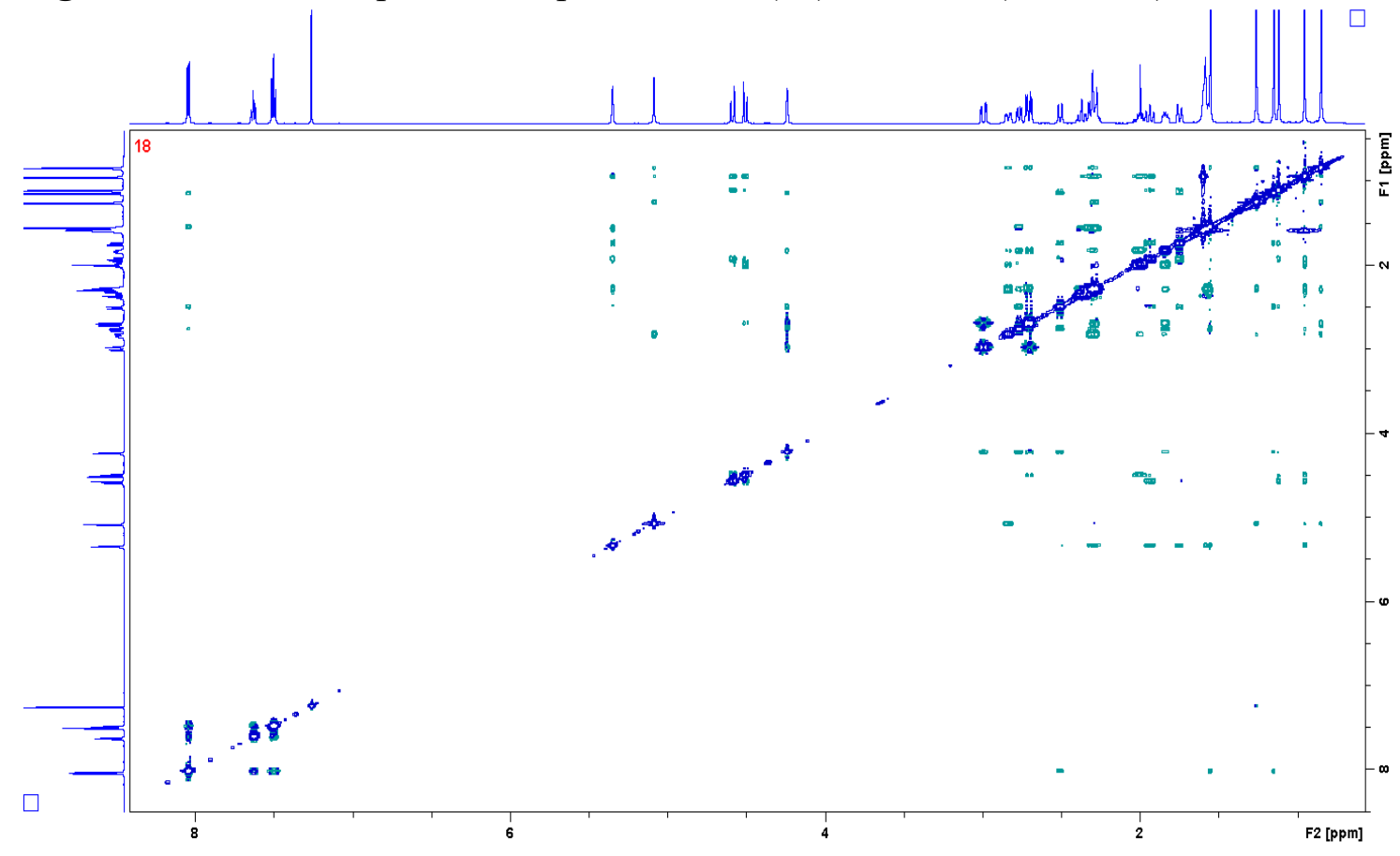

Figure S74. Expansion of the NOESY spectrum of picraviane R (18) in $\mathrm{CDCl}_{3}(600 \mathrm{MHz})$.

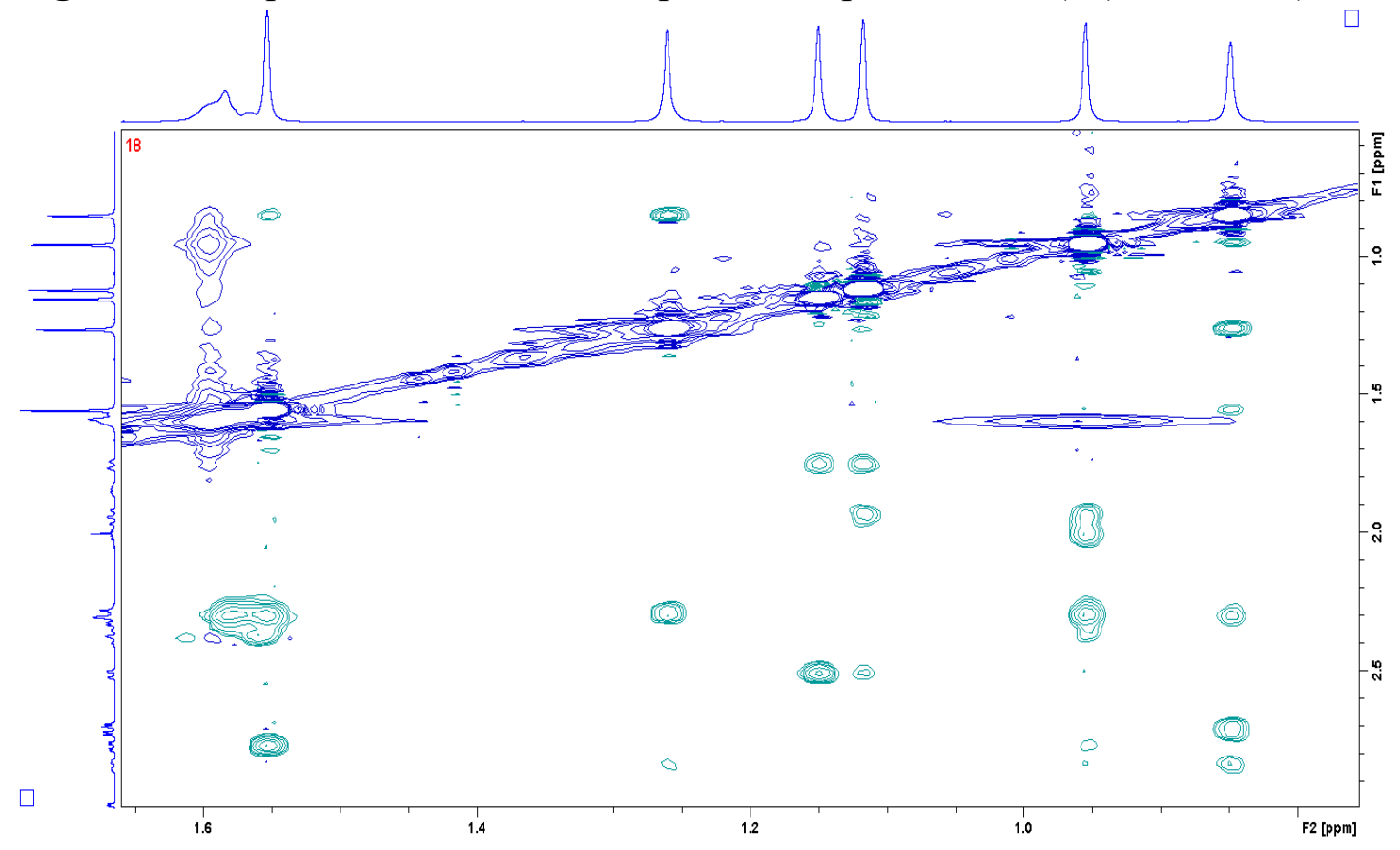


Figure S75. ${ }^{1} \mathrm{H}$ NMR spectrum of picraviane $\mathrm{S}(19)$ in $\mathrm{CDCl}_{3}(600 \mathrm{MHz})$.

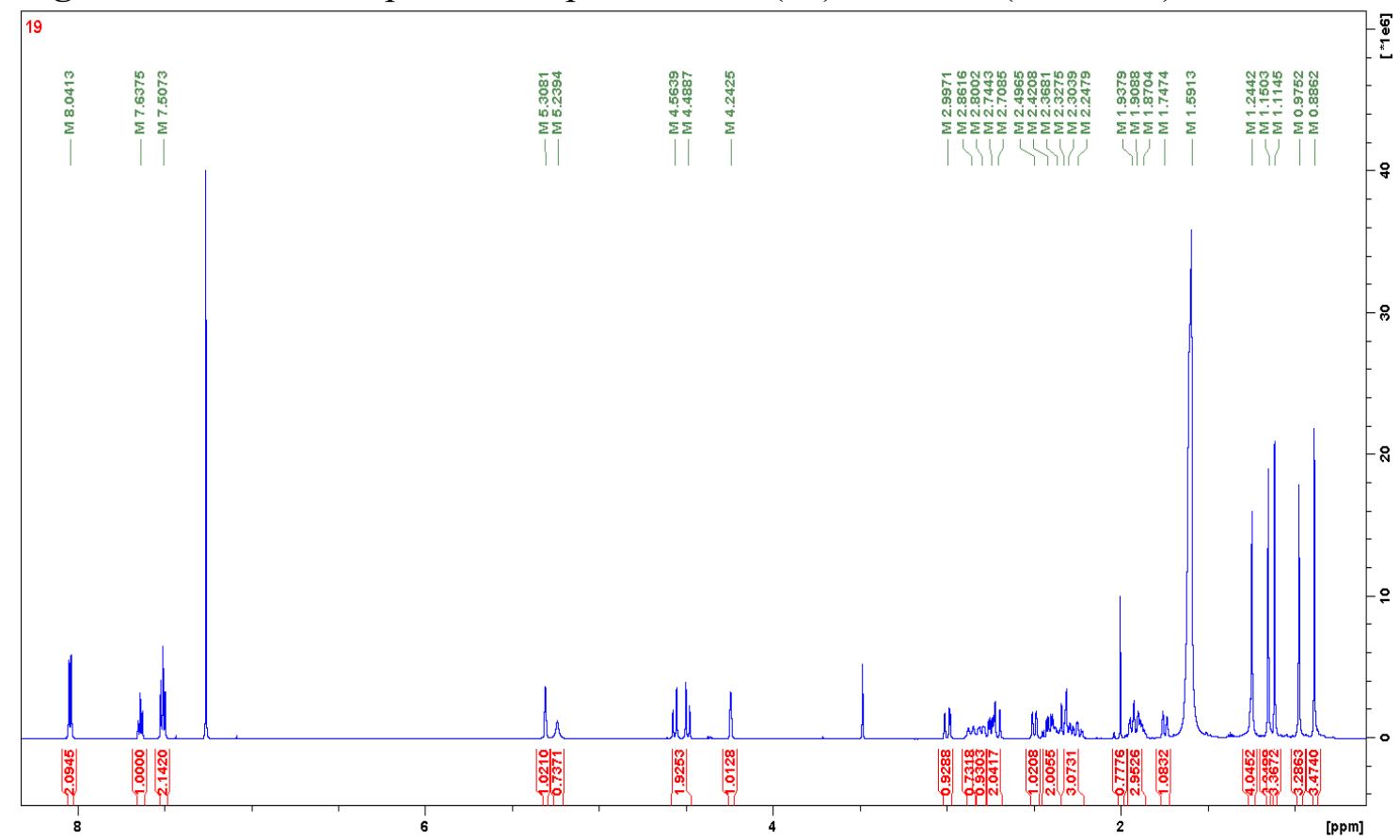

Figure S76. Expansion of the ${ }^{1} \mathrm{H}$ NMR spectrum of picraviane $\mathrm{S}(19)$ in $\mathrm{CDCl}_{3}(600 \mathrm{MHz})$.

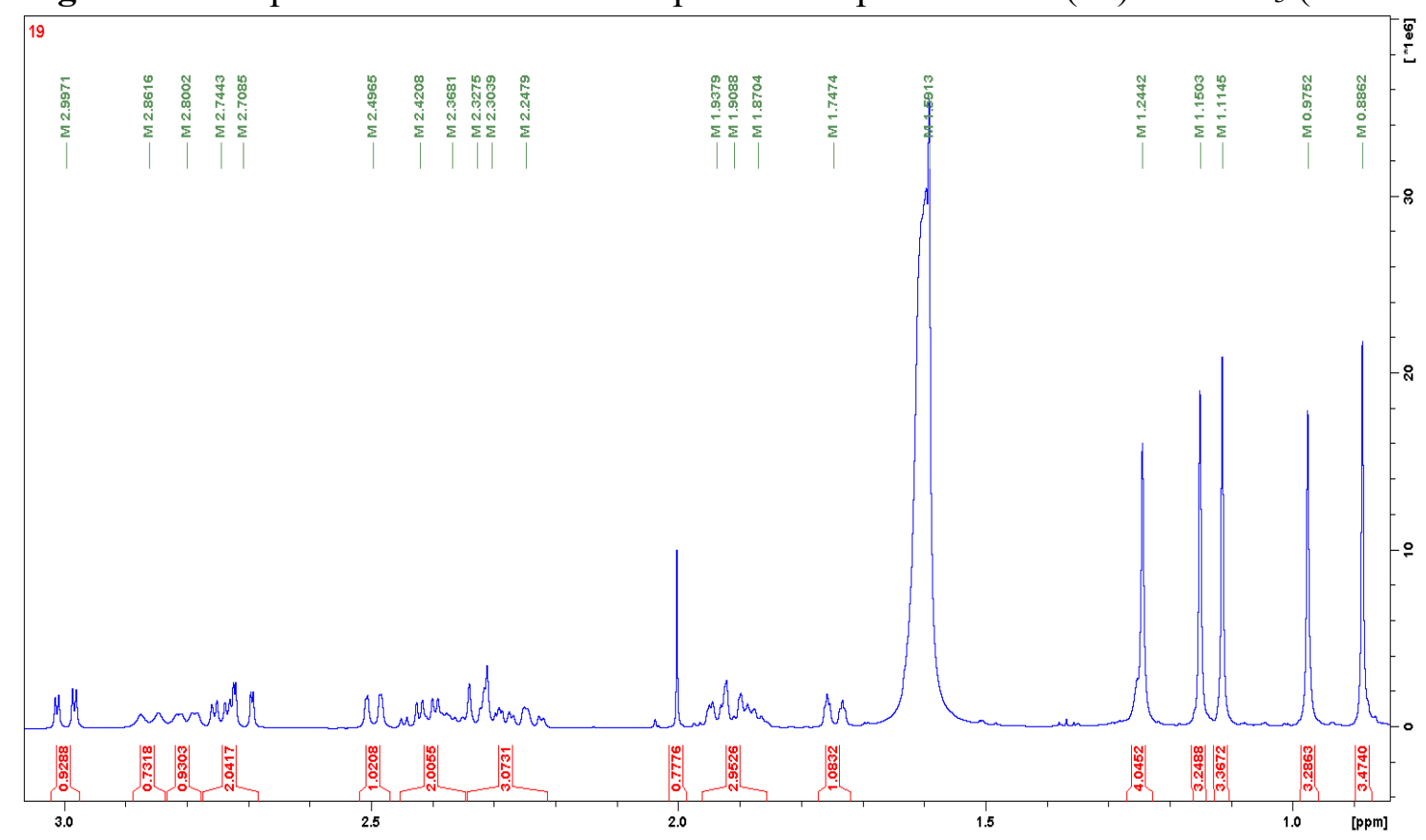


Figure S77. ${ }^{13} \mathrm{C}$ NMR spectrum of picraviane $\mathrm{S}(19)$ in $\mathrm{CDCl}_{3}(150 \mathrm{MHz})$.

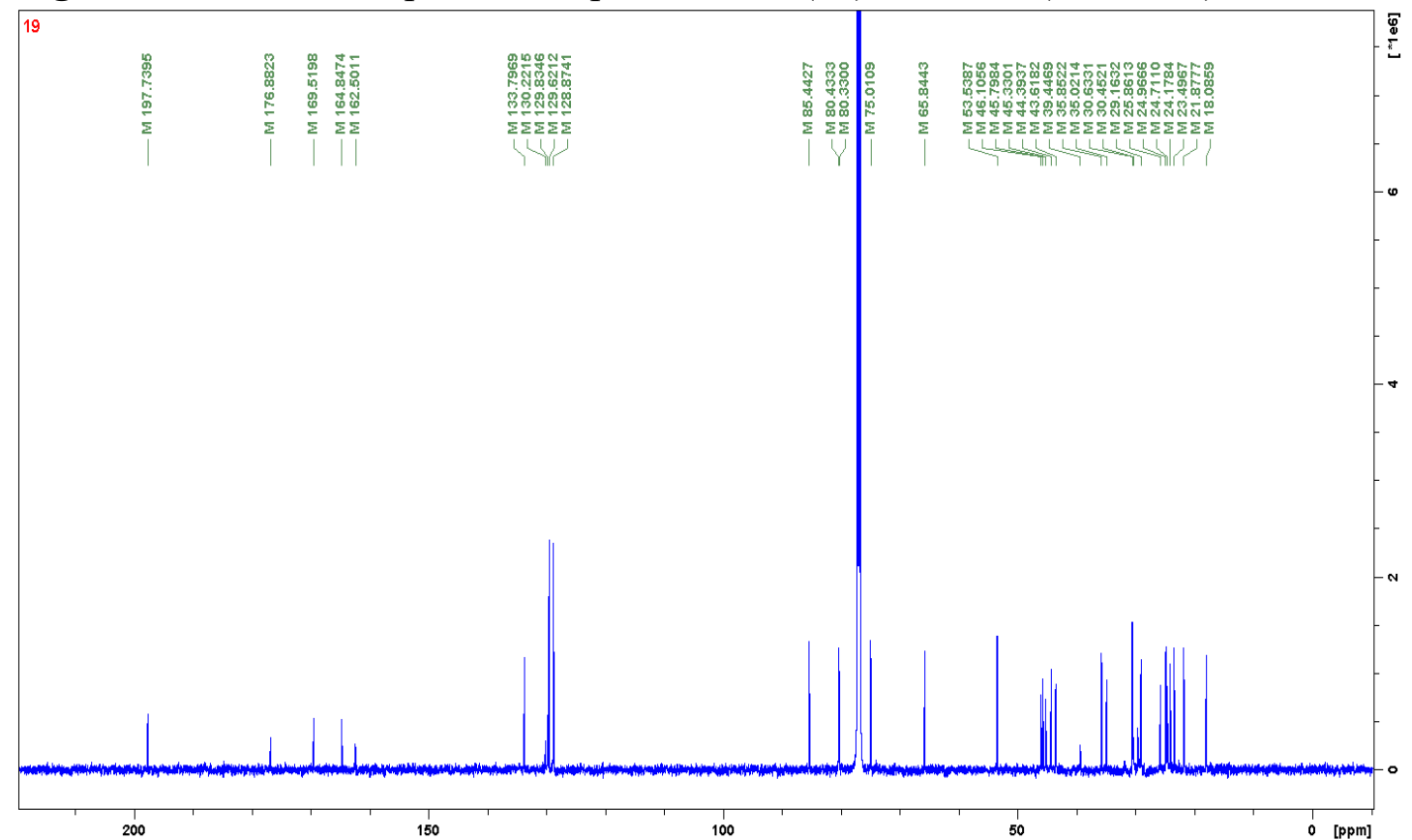

Figure S78. HSQC spectrum of picraviane $\mathrm{S}(19)$ in $\mathrm{CDCl}_{3}(600 \mathrm{MHz})$.

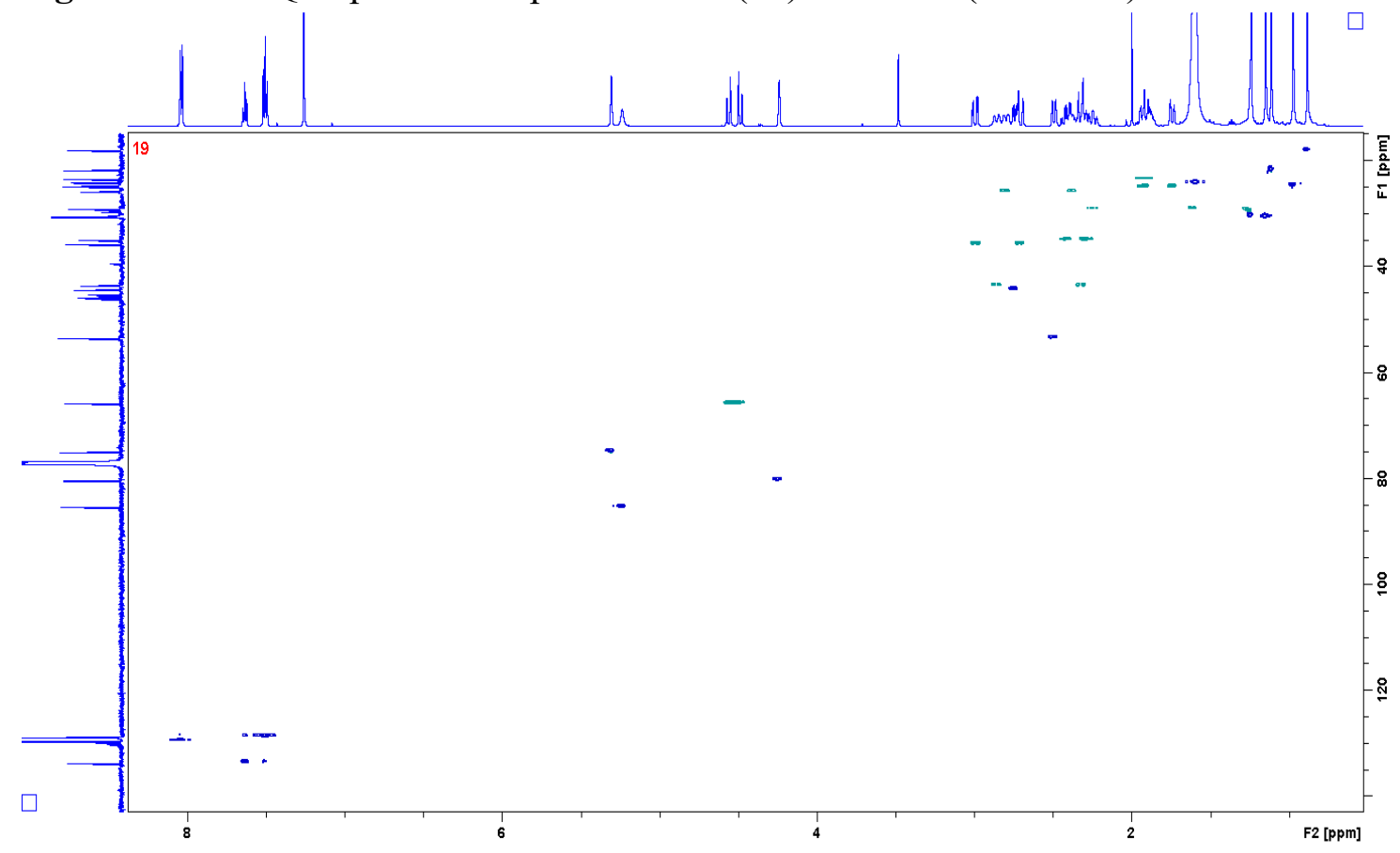


Figure S79. HMBC spectrum of picraviane $\mathrm{S}(19)$ in $\mathrm{CDCl}_{3}(600 \mathrm{MHz})$.

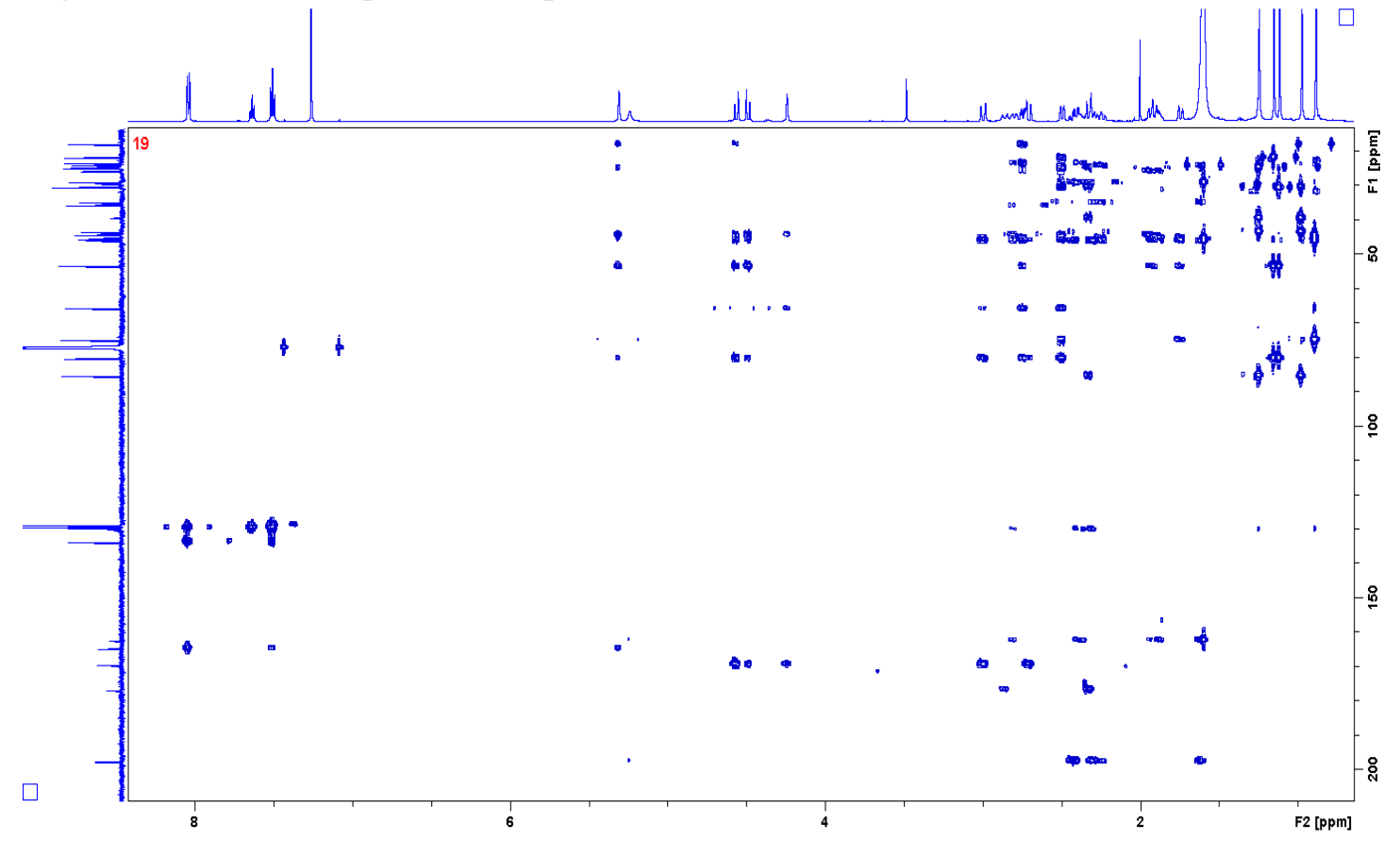

Figure S80. COSY spectrum of picraviane $\mathrm{S}(19)$ in $\mathrm{CDCl}_{3}(600 \mathrm{MHz})$.

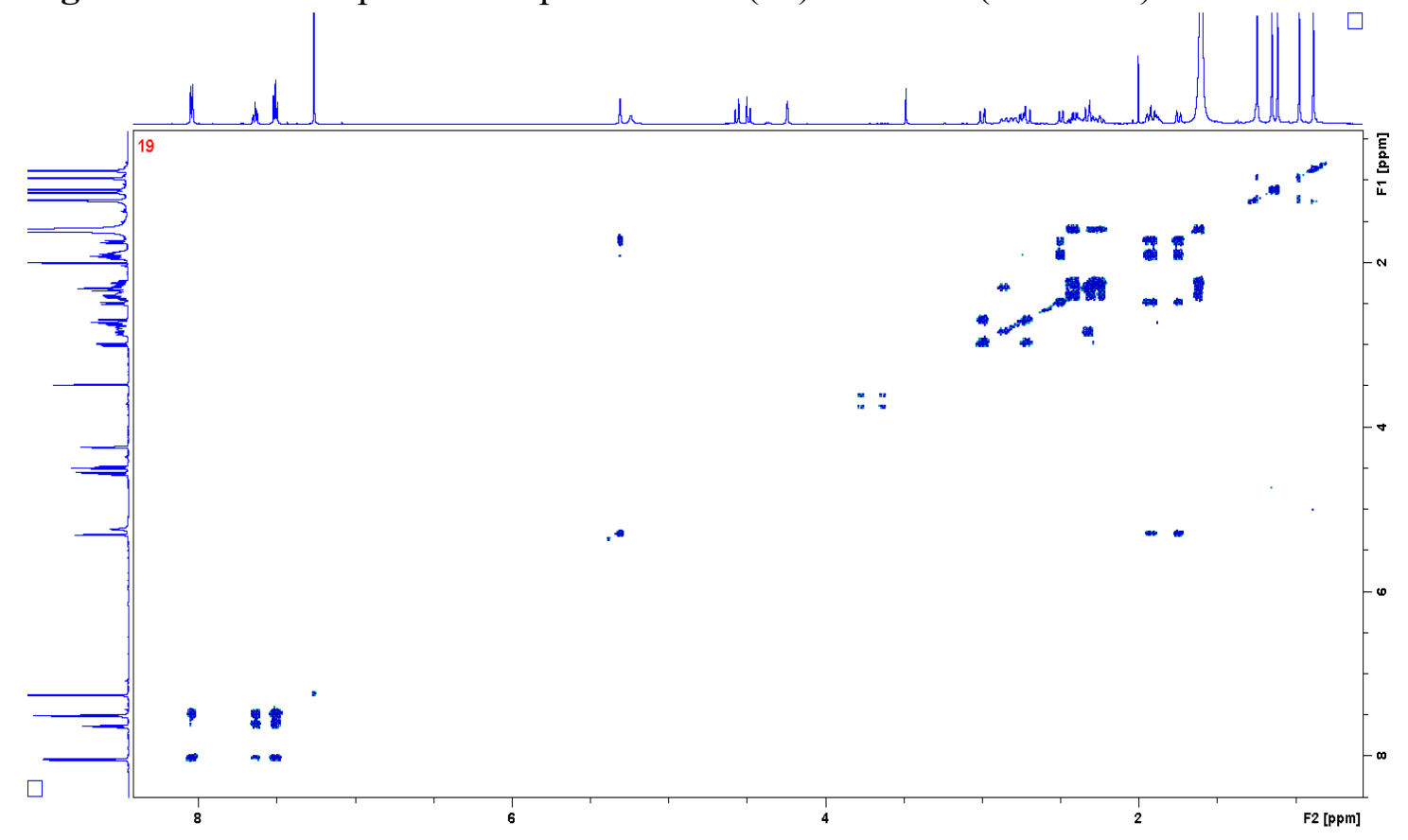


Figure S81. NOESY spectrum of picraviane $\mathrm{S}(19)$ in $\mathrm{CDCl}_{3}(600 \mathrm{MHz})$.

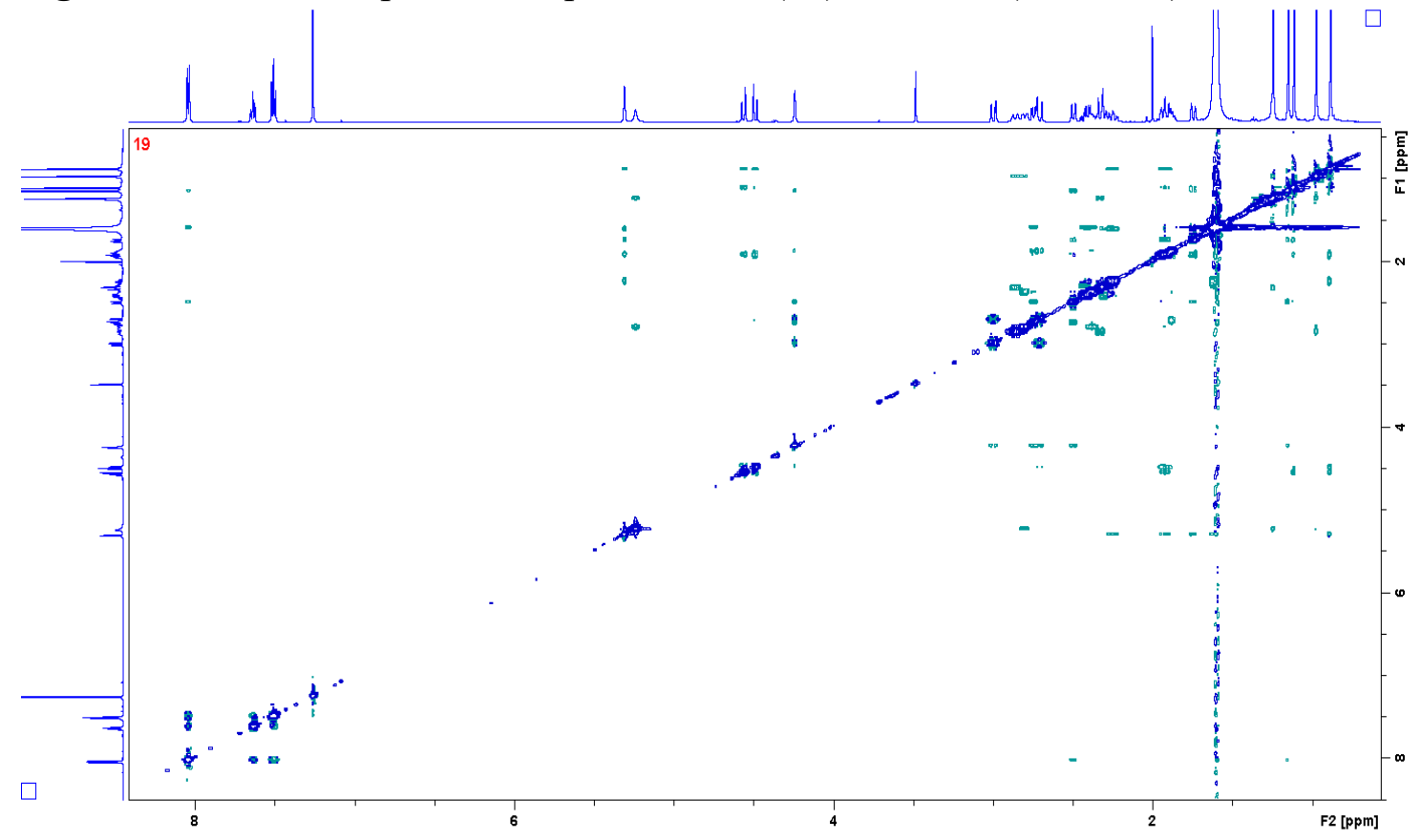

Figure S82. Expansion of the NOESY spectrum of picraviane $\mathrm{S}(\mathbf{1 9})$ in $\mathrm{CDCl}_{3}(600 \mathrm{MHz})$.

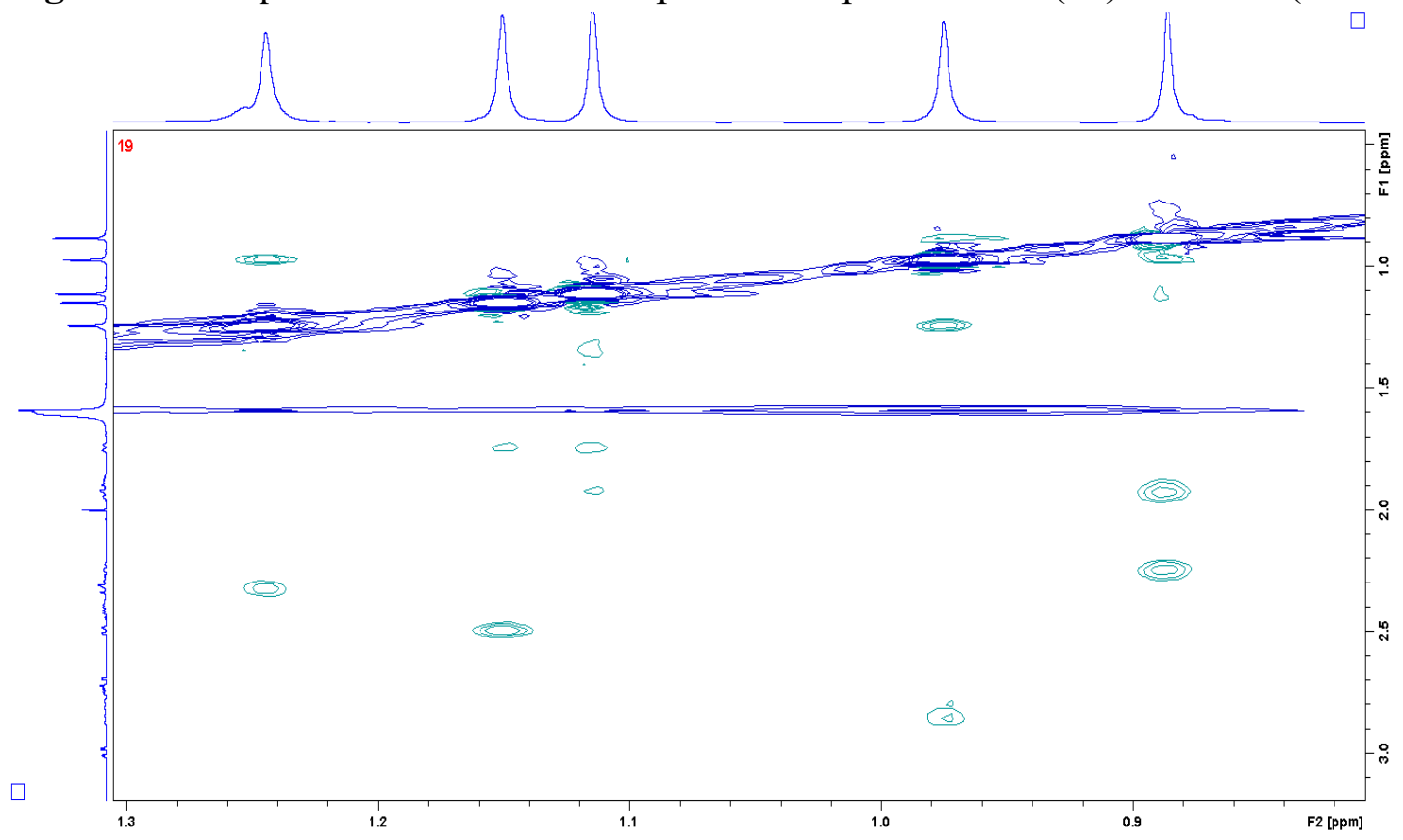


Figure S83. Structures of the two lowest-energy conformers of $(1 S, 5 R, 7 R, 8 R, 9 S, 10 R, 14 R, 19 S)-18$ at the B3LYP/PCM(MeOH)/TZVP level. The simple average of the two conformers was used for calculation of the ECD spectrum.

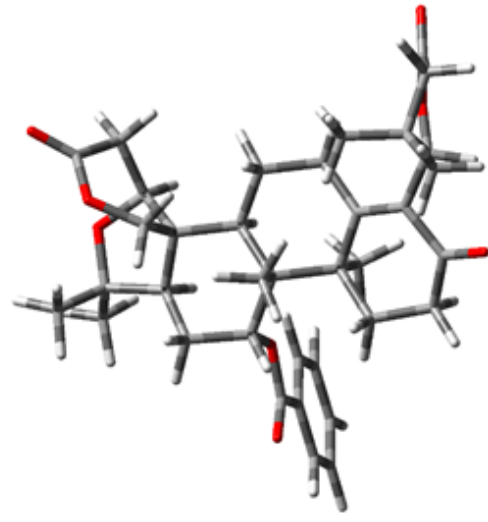

Conf. 1

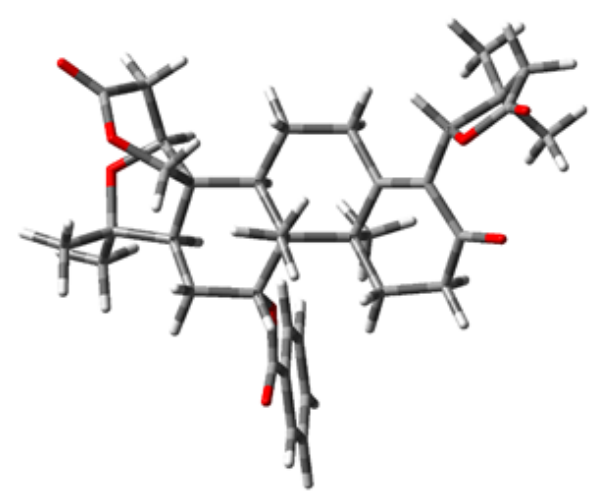

Conf. 2

Figure S84. Comparison of the experimental and calculated UV and ECD spectra of $(1 S, 5 R, 7 R, 8 R, 9 S, 10 R, 14 R, 19 S)-\mathbf{1 8}$ (blue trace) and its enantiomer (red trace). Experimental spectra were acquired in methanol, and UV and ECD spectra were calculated at the [CAM$\mathrm{B} 3 \mathrm{LYP} / \mathrm{PCM}(\mathrm{MeOH}) / \mathrm{TZVP}]$ level for the simple average of the two lowest-energy conformers identified.

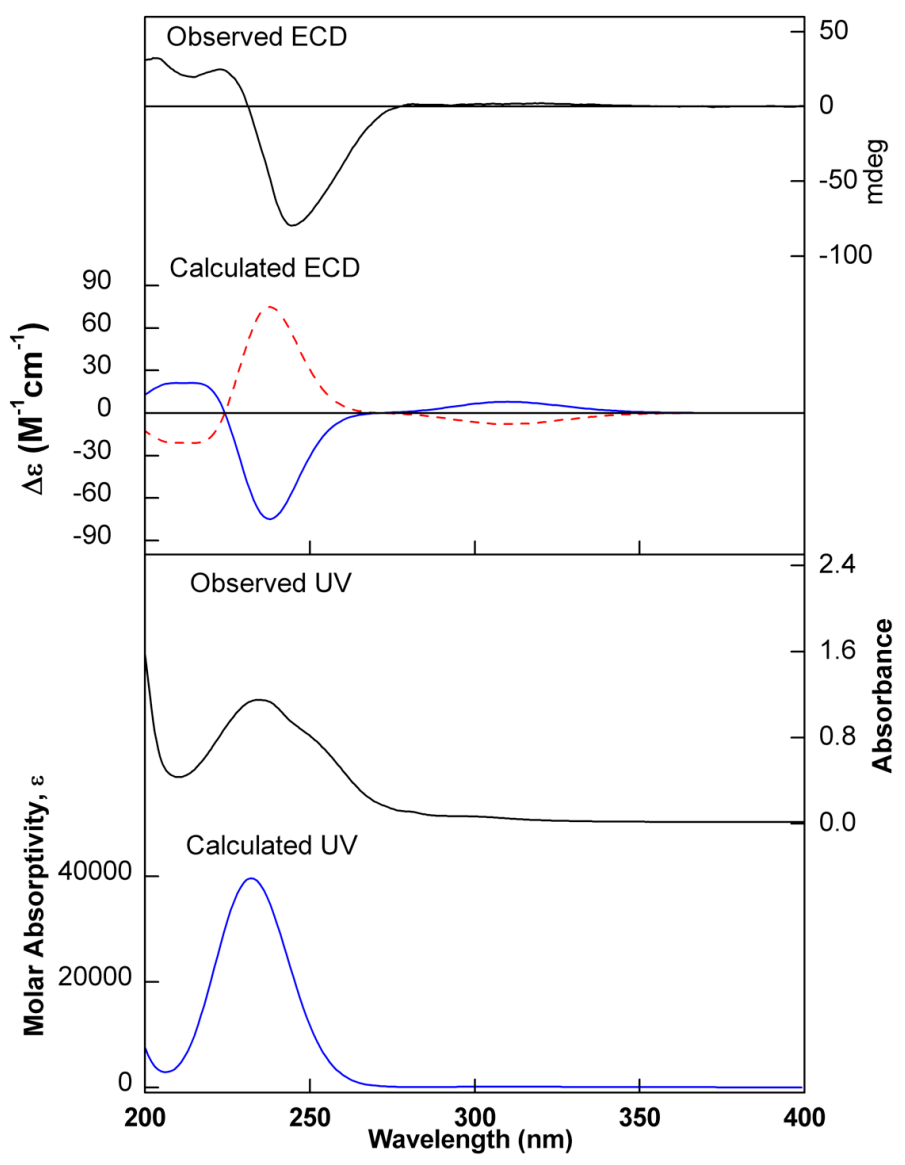


Figure S85. Structures of the two lowest-energy conformers of $(1 S, 5 R, 7 R, 8 R, 9 S, 10 R, 14 R, 19 R)-19$ at the B3LYP/PCM(MeOH)/TZVP level. The simple average of the two conformers was used for calculation of the ECD spectrum.

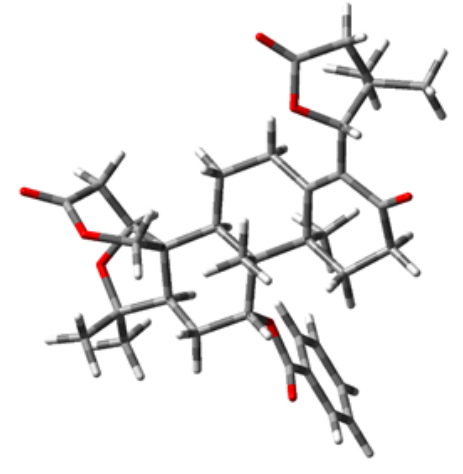

Conf. 1

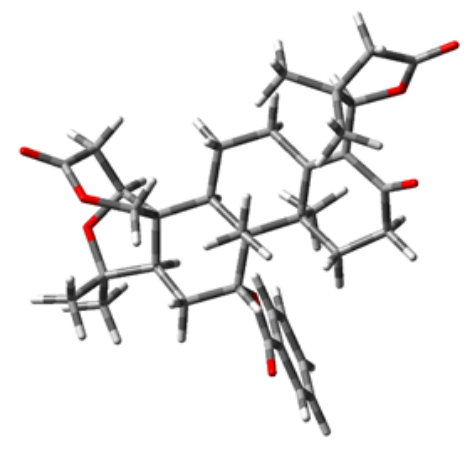

Conf. 2

Figure S86. Comparison of the experimental and calculated UV and ECD spectra of $(1 S, 5 R, 7 R, 8 R, 9 S, 10 R, 14 R, 19 R)-19$ (blue trace) and its enantiomer (red trace). Experimental spectra were acquired in methanol, and UV and ECD spectra were calculated at the [CAM$\mathrm{B} 3 \mathrm{LYP} / \mathrm{PCM}(\mathrm{MeOH}) / \mathrm{TZVP}]$ level for the simple average of the two lowest-energy conformers identified.

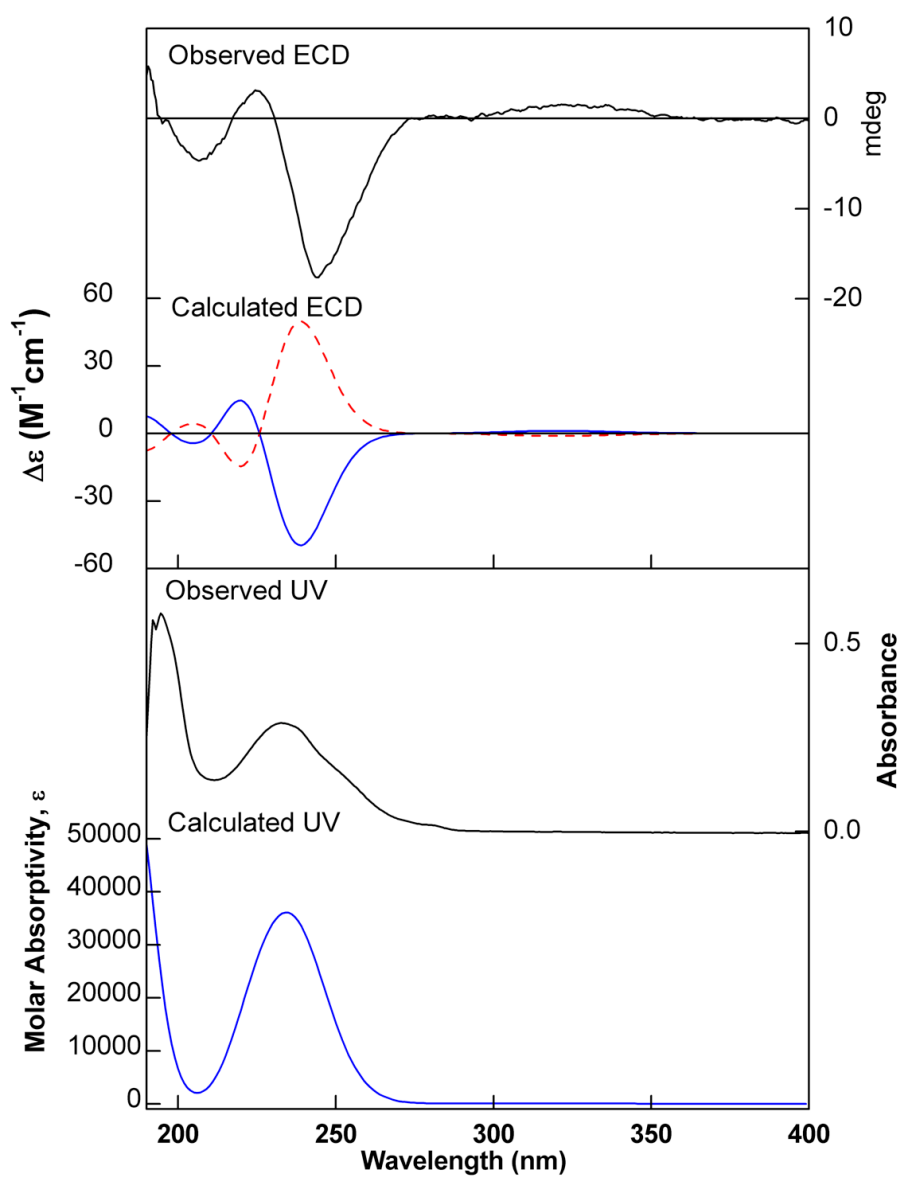


Figure S87. ${ }^{1} \mathrm{H}$ NMR spectrum of picraviane F (5) and the coeluting anthraquinone aloeemodin in $\mathrm{CDCl}_{3}(600 \mathrm{MHz})$.

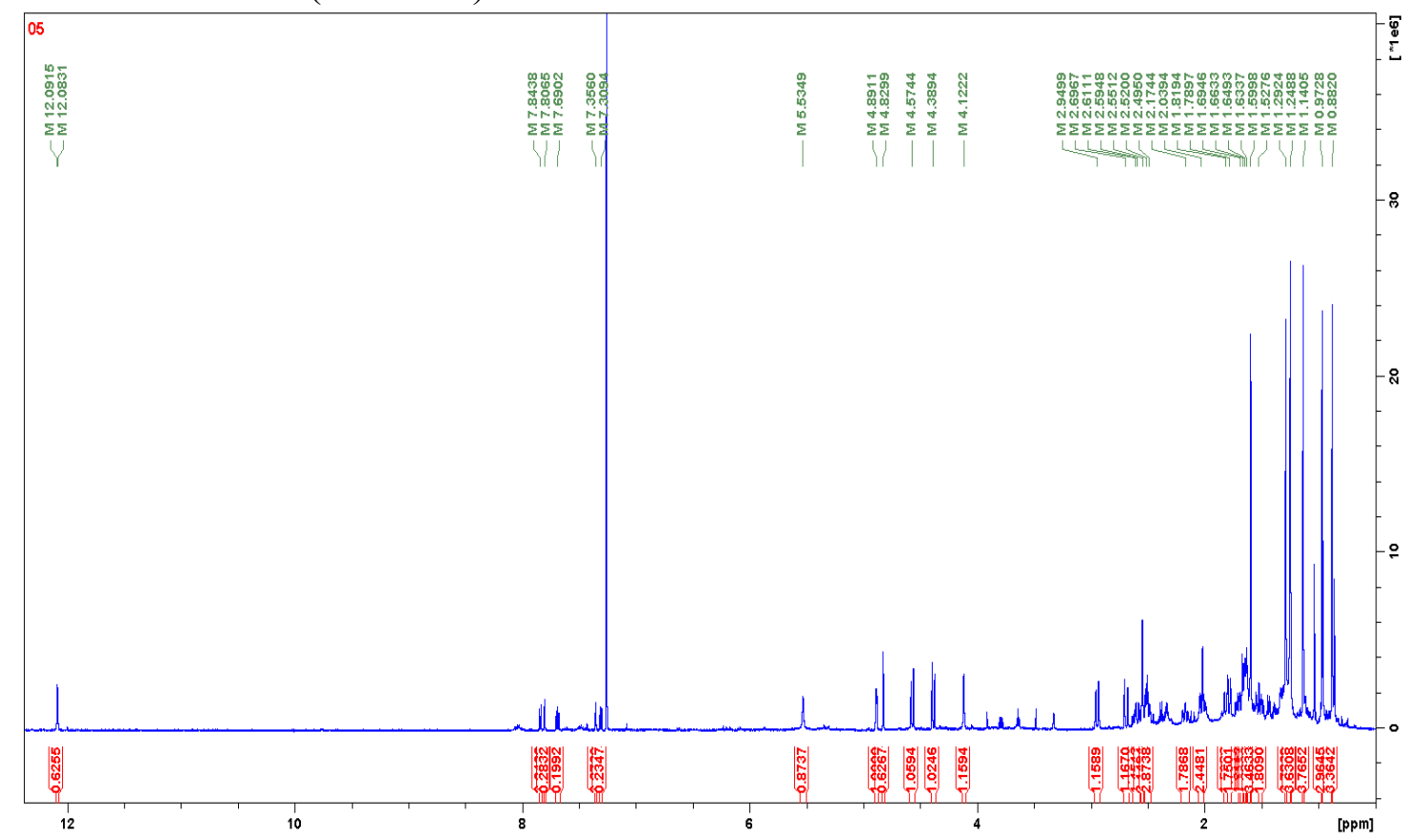

Figure S88. Expansion of the ${ }^{1} \mathrm{H}$ NMR spectrum of picraviane F (5) and the coeluting anthraquinone aloe-emodin in $\mathrm{CDCl}_{3}(600 \mathrm{MHz})$.

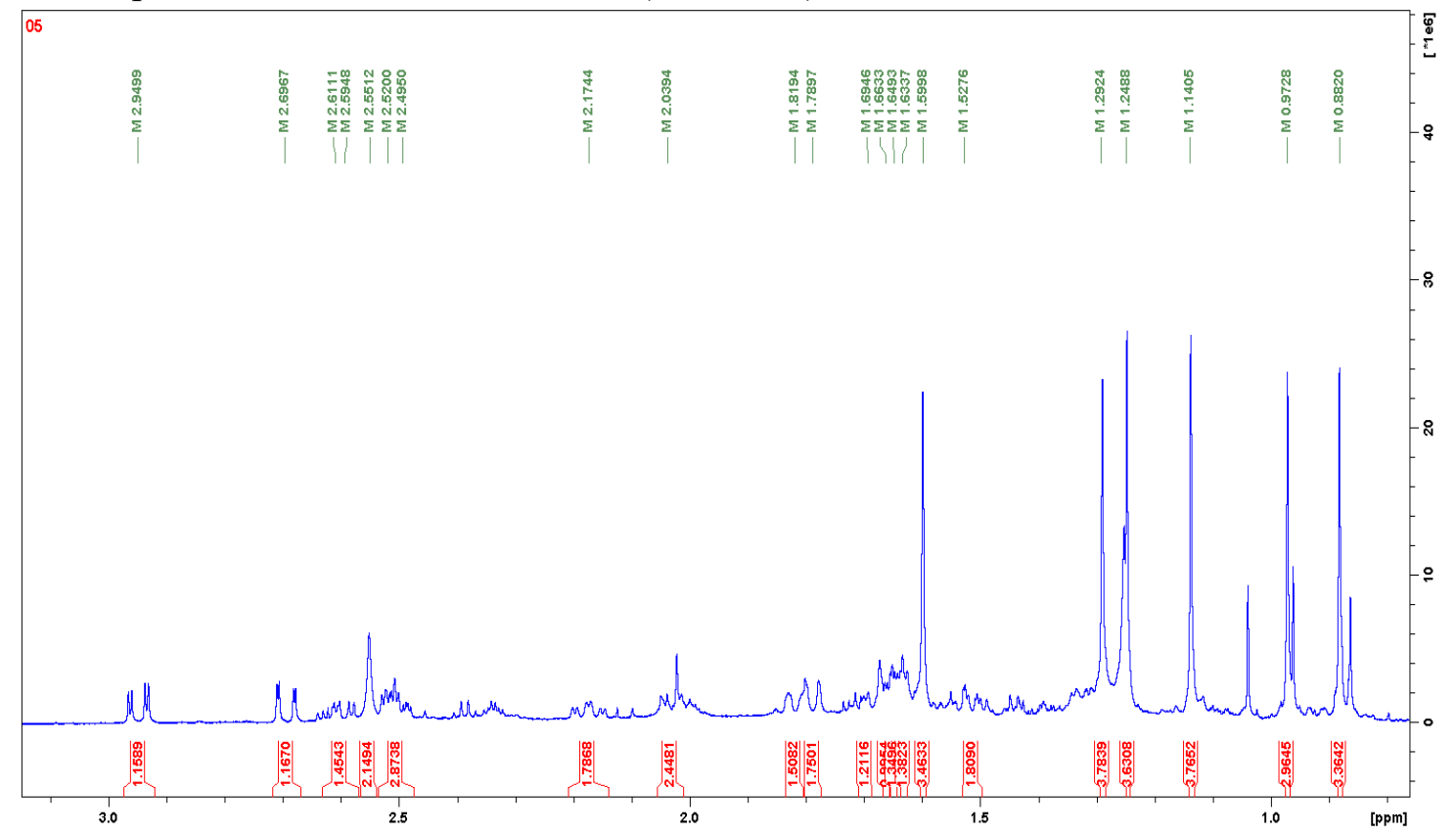


Figure S89. ${ }^{13} \mathrm{C}$ NMR spectrum of picraviane F (5) and the coeluting anthraquinone aloeemodin in $\mathrm{CDCl}_{3}(150 \mathrm{MHz})$.

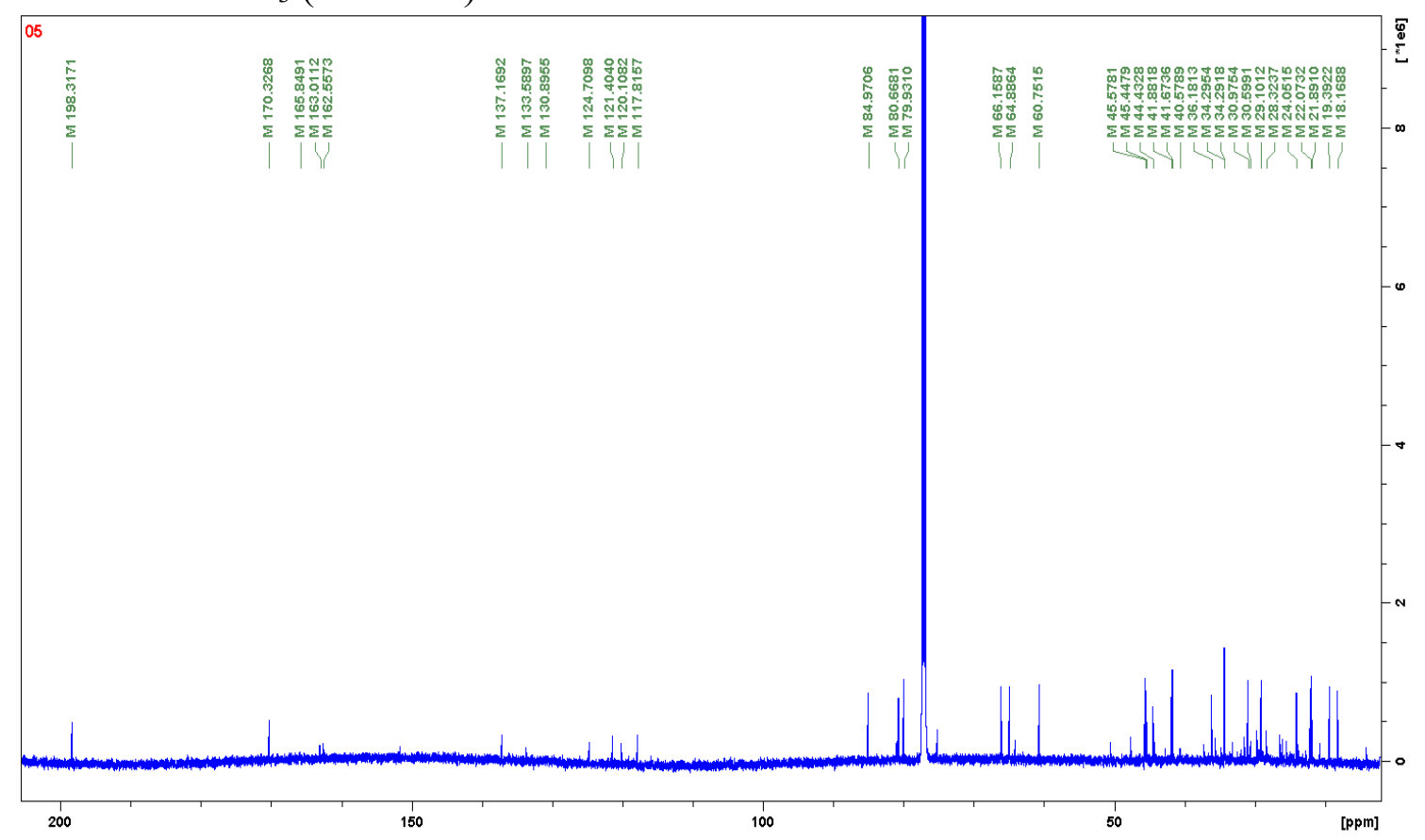

Figure S90. HSQC spectrum of picraviane F (5) and the coeluting anthraquinone aloeemodin in $\mathrm{CDCl}_{3}(600 \mathrm{MHz})$.

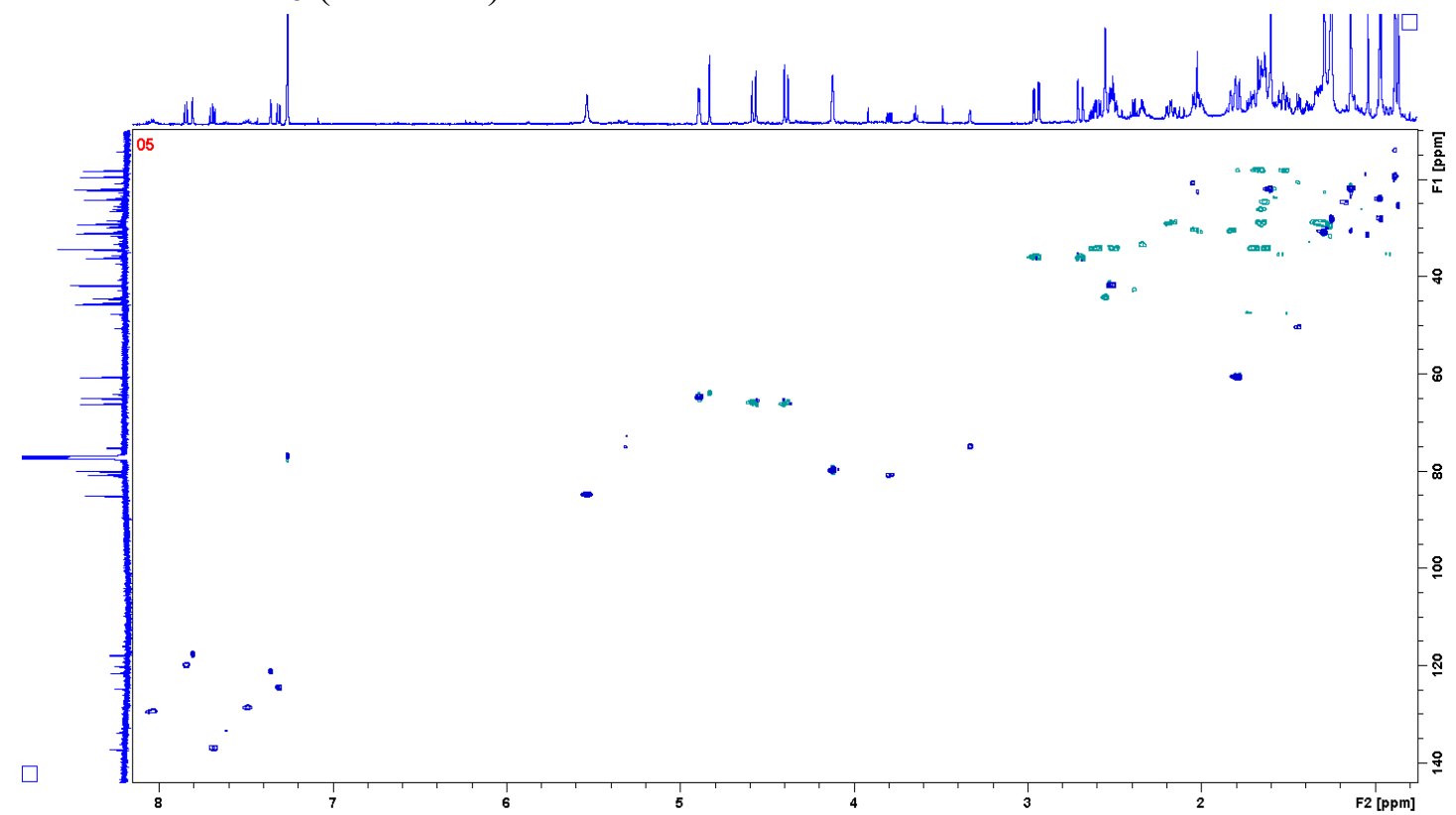


Figure S91. HMBC spectrum of picraviane F (5) and the coeluting anthraquinone aloeemodin in $\mathrm{CDCl}_{3}(600 \mathrm{MHz})$.

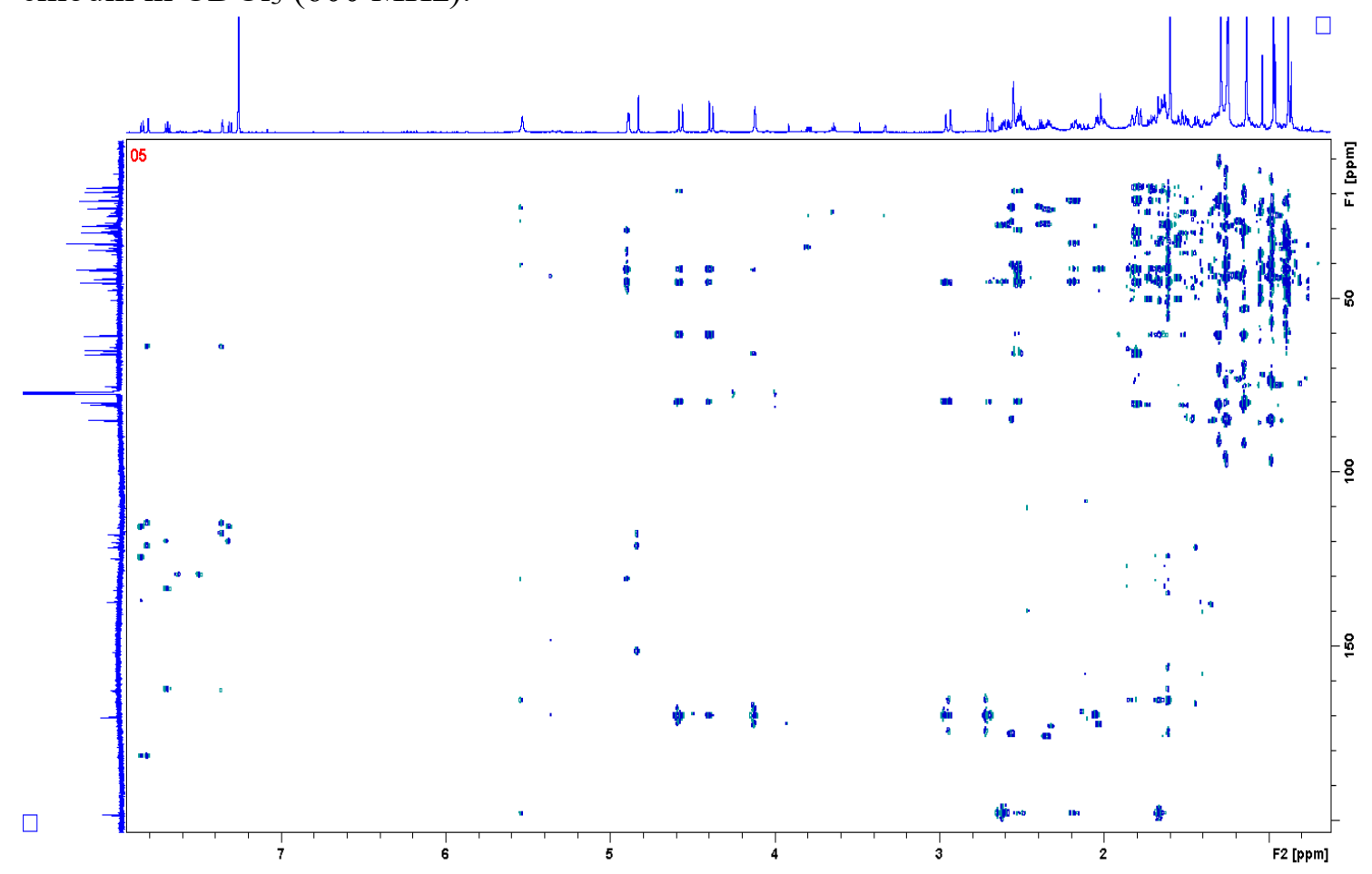

Figure S92. COSY spectrum of picraviane F (5) and the coeluting anthraquinone aloeemodin in $\mathrm{CDCl}_{3}(600 \mathrm{MHz})$.

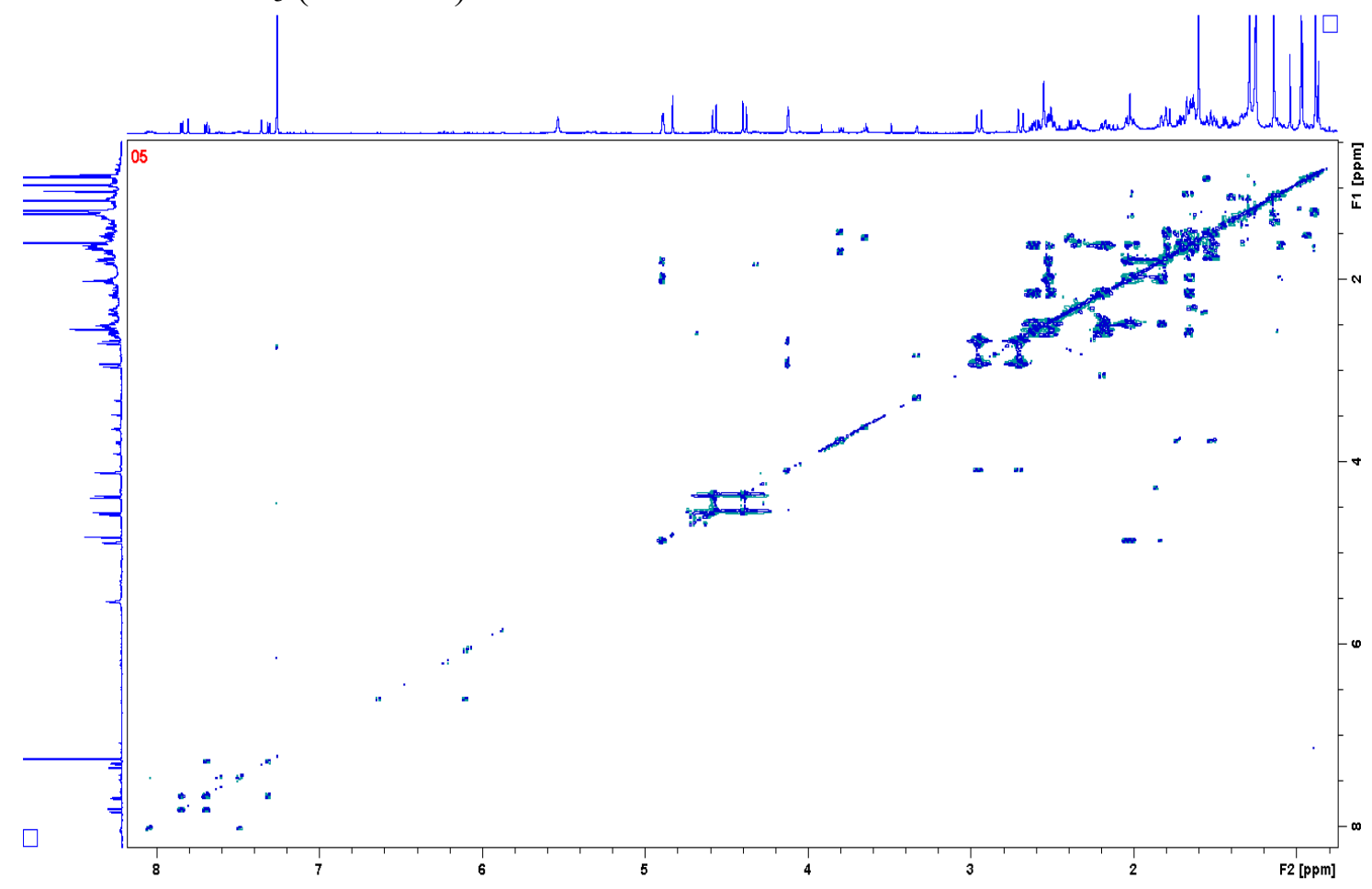


Figure S93. NOESY spectrum of picraviane F (5) and the coeluting anthraquinone aloeemodin in $\mathrm{CDCl}_{3}(600 \mathrm{MHz})$.

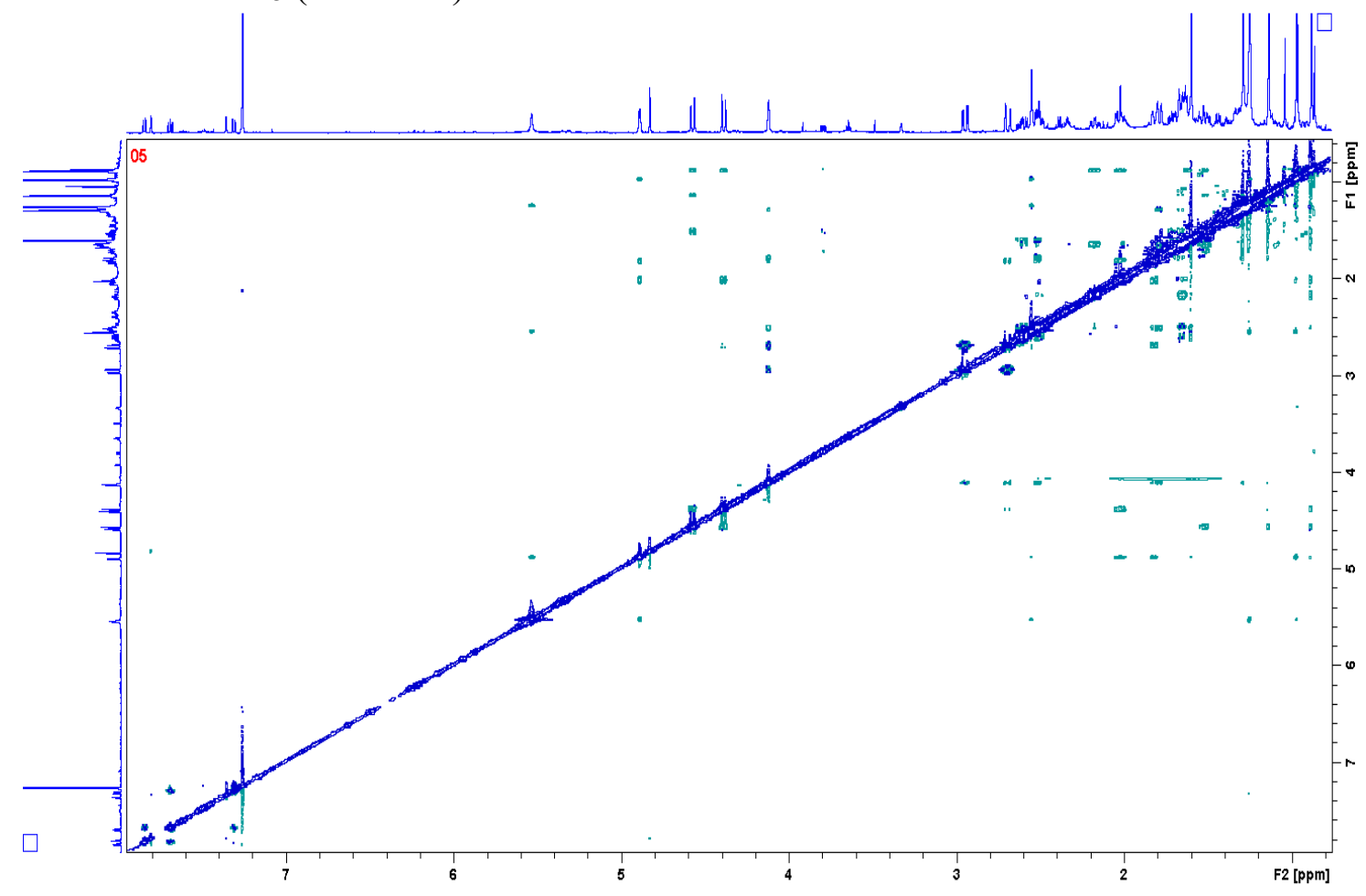

Figure S94. ${ }^{1} \mathrm{H}$ NMR spectrum of picraviane $\mathrm{L}(\mathbf{1 2})$ in $\mathrm{CDCl}_{3}(600 \mathrm{MHz})$.

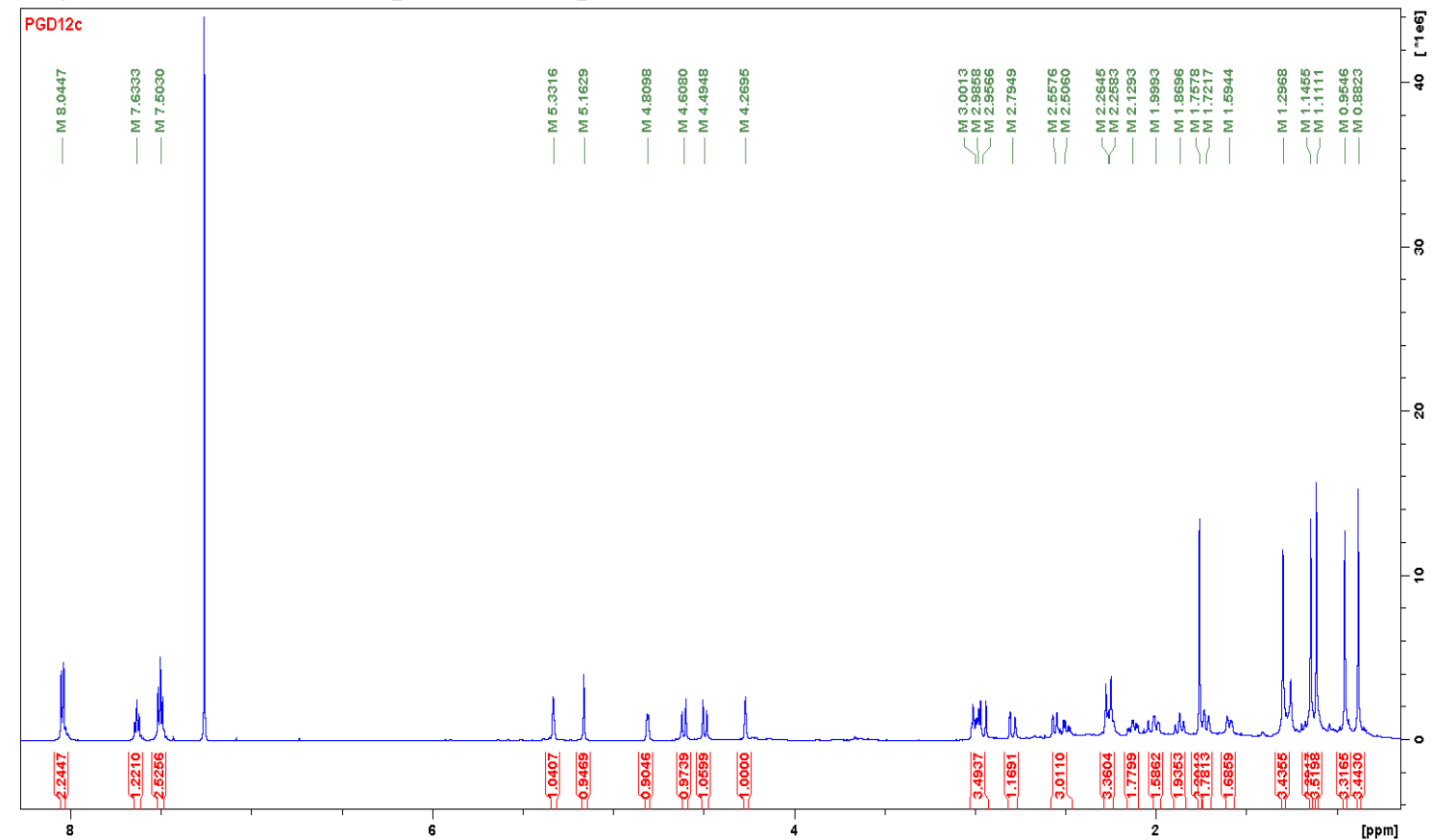


Figure S95. Expansion of the ${ }^{1} \mathrm{H}$ NMR spectrum of picraviane $\mathrm{L}(12)$ in $\mathrm{CDCl}_{3}(600 \mathrm{MHz})$.

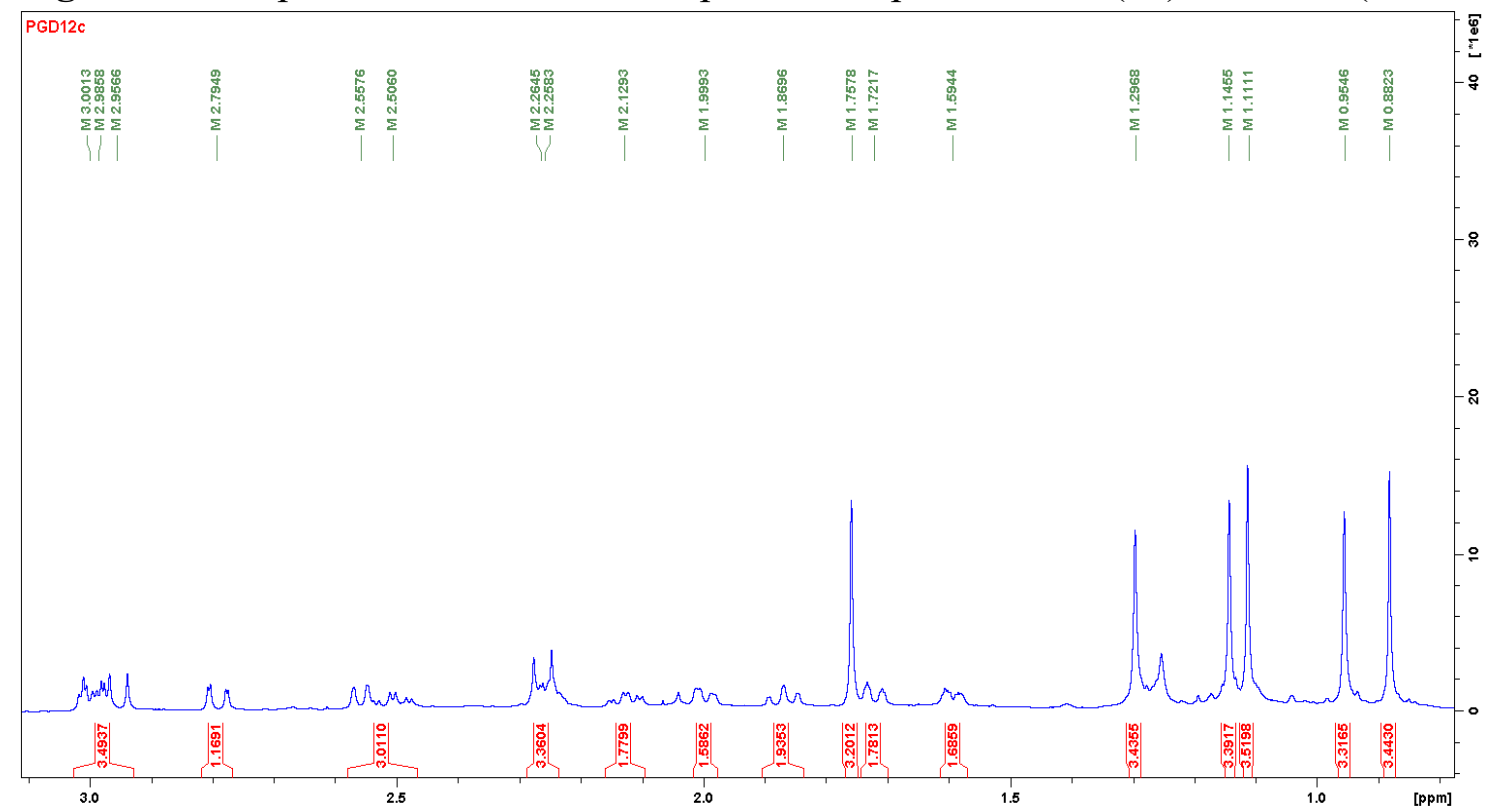

Figure S96. ${ }^{13} \mathrm{C}$ NMR spectrum of picraviane $\mathrm{L}(\mathbf{1 2})$ in $\mathrm{CDCl}_{3}(150 \mathrm{MHz})$.

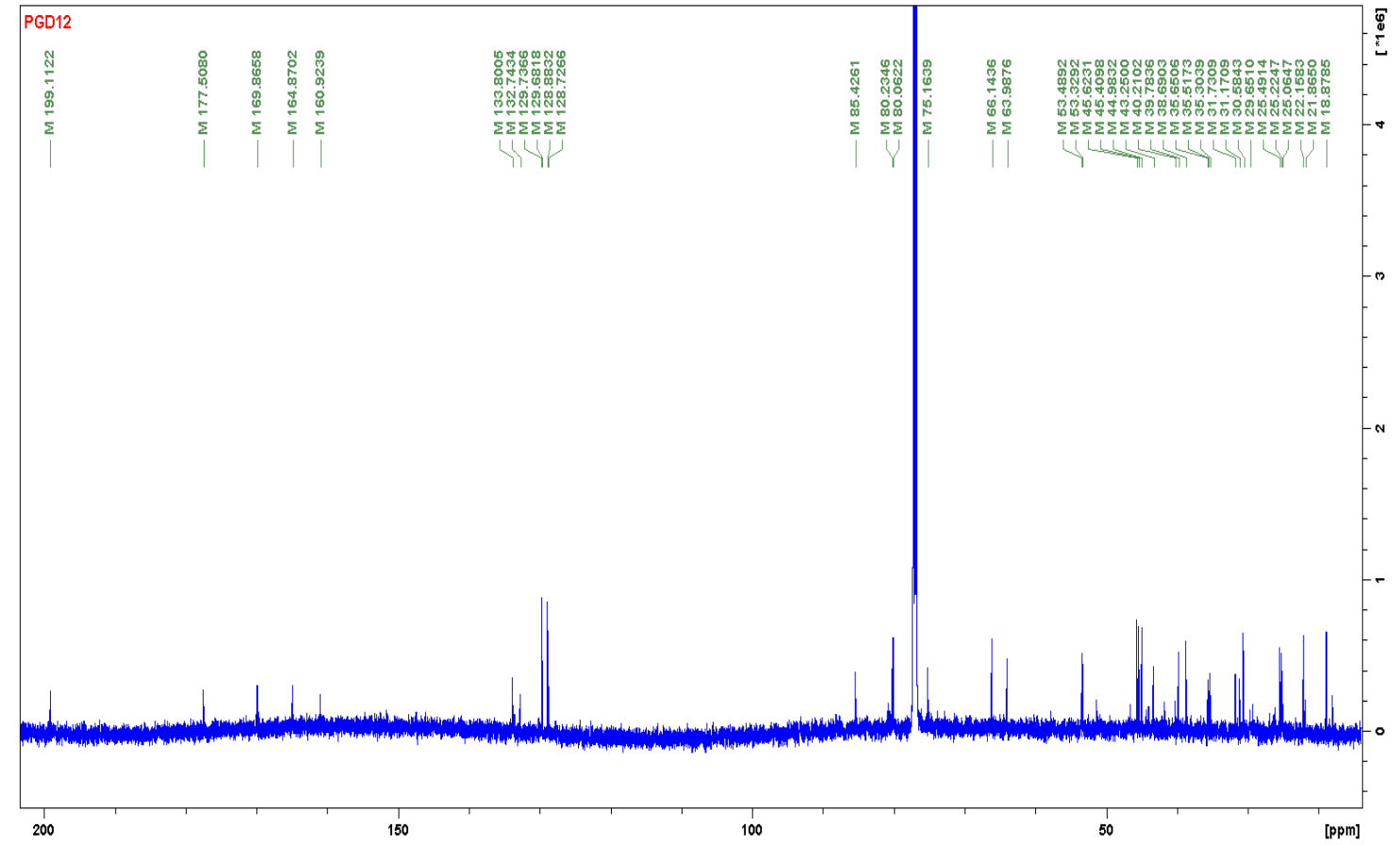


Figure S97. HSQC spectrum of picraviane L (12) in $\mathrm{CDCl}_{3}(600 \mathrm{MHz})$.

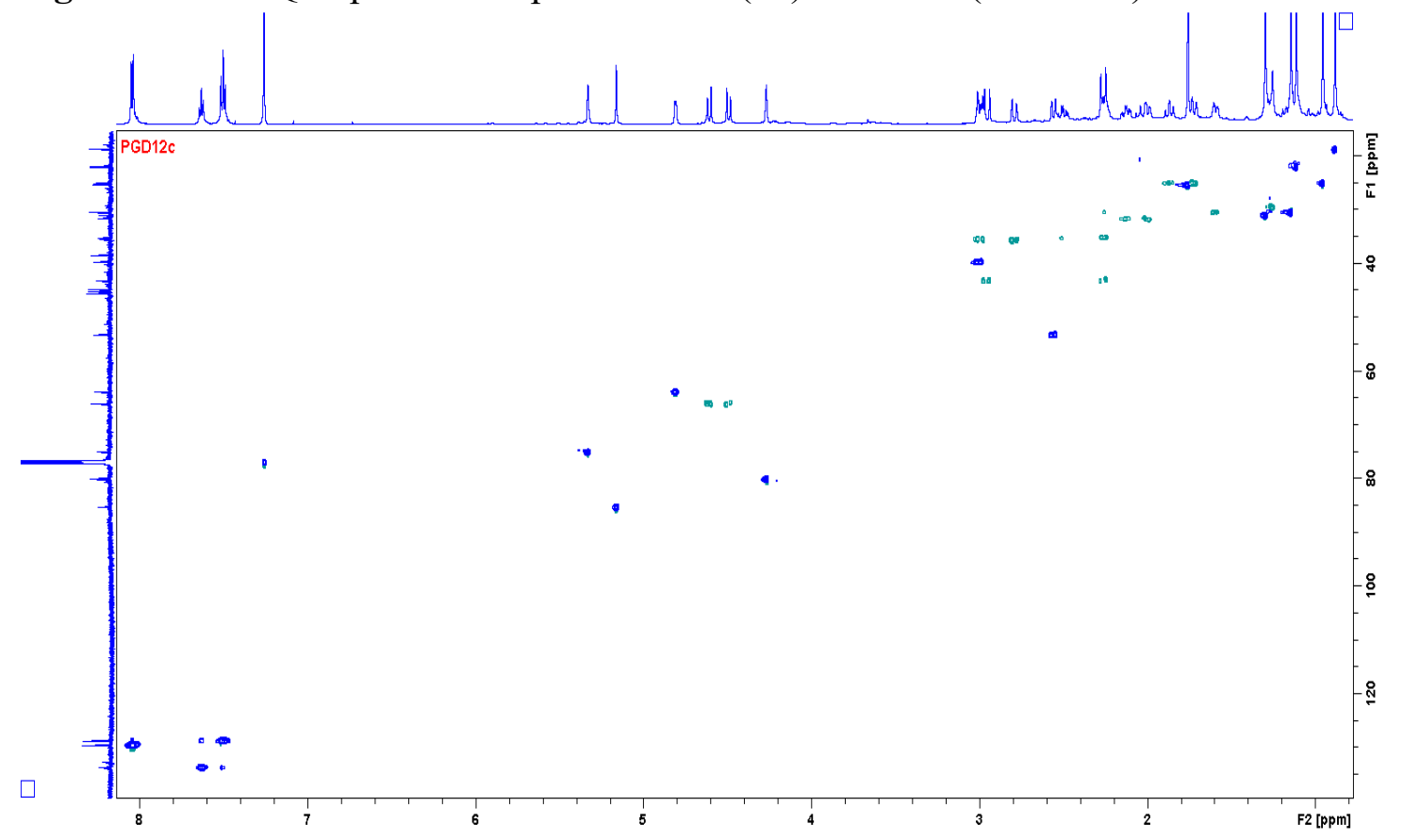

Figure S98. HMBC spectrum of picraviane $\mathrm{L}(12)$ in $\mathrm{CDCl}_{3}(600 \mathrm{MHz})$.

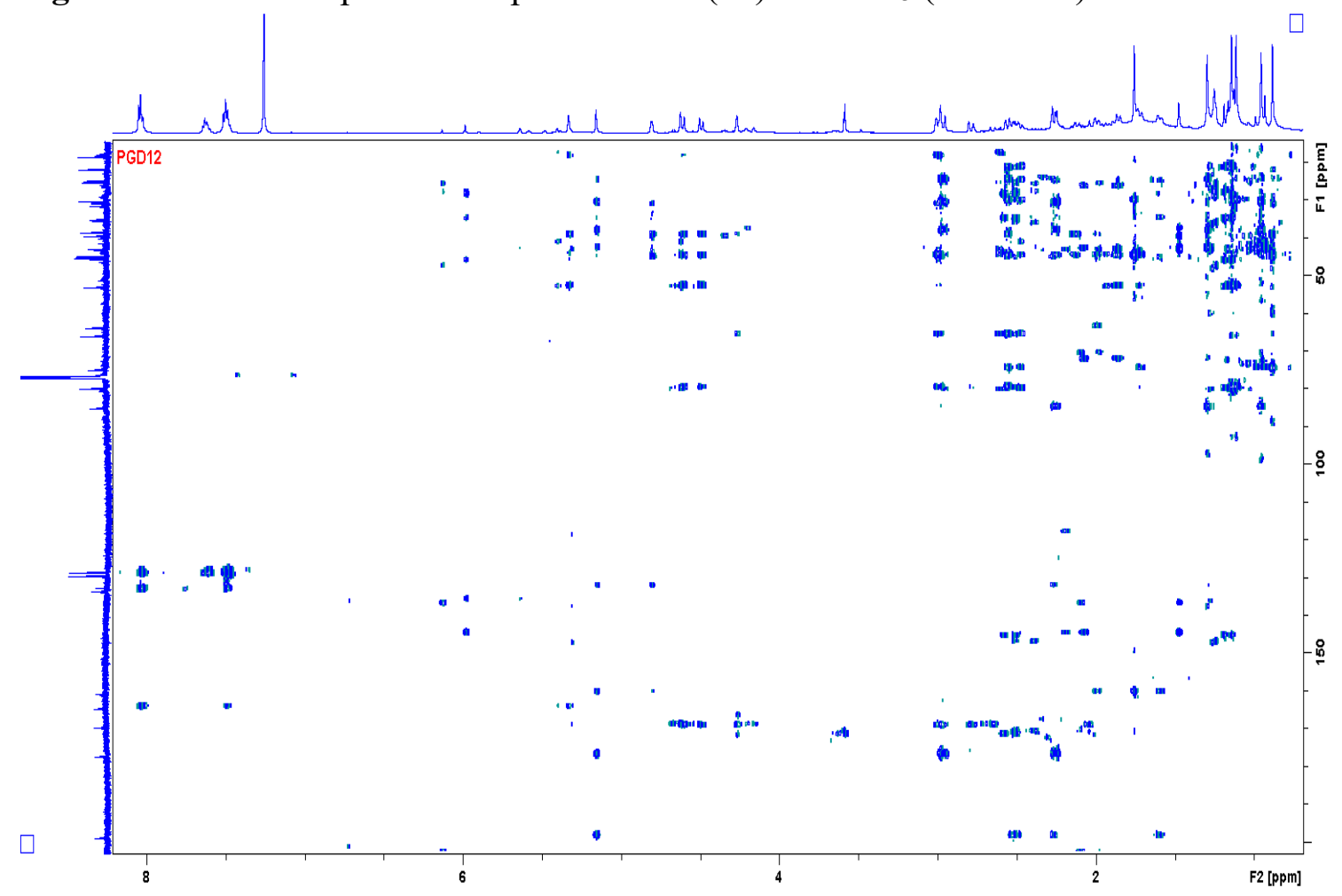


Figure S99. COSY spectrum of picraviane L (12) in $\mathrm{CDCl}_{3}(600 \mathrm{MHz})$.

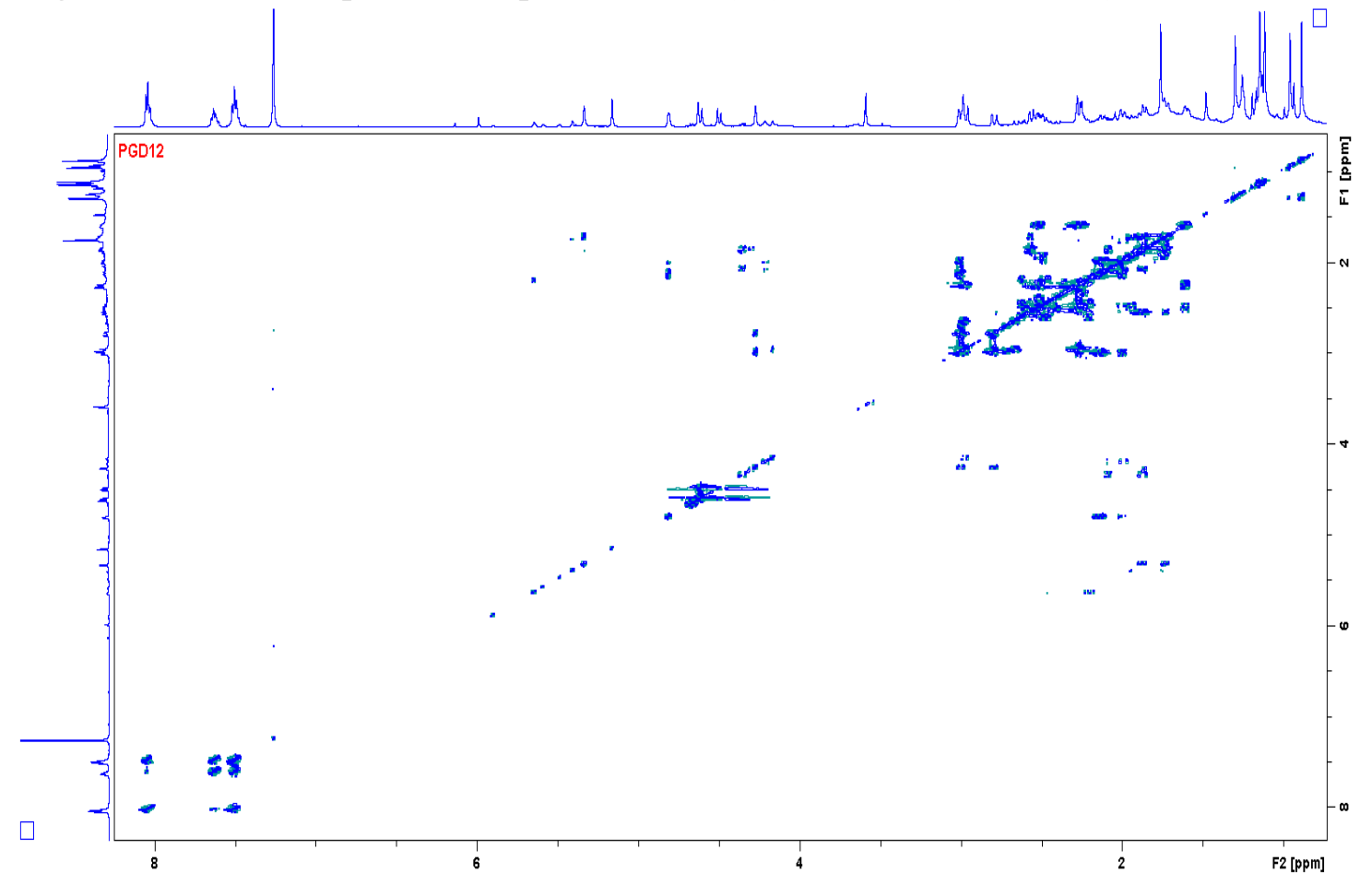

Figure S100. NOESY spectrum of picraviane $\mathrm{L}(12)$ in $\mathrm{CDCl}_{3}(600 \mathrm{MHz})$.

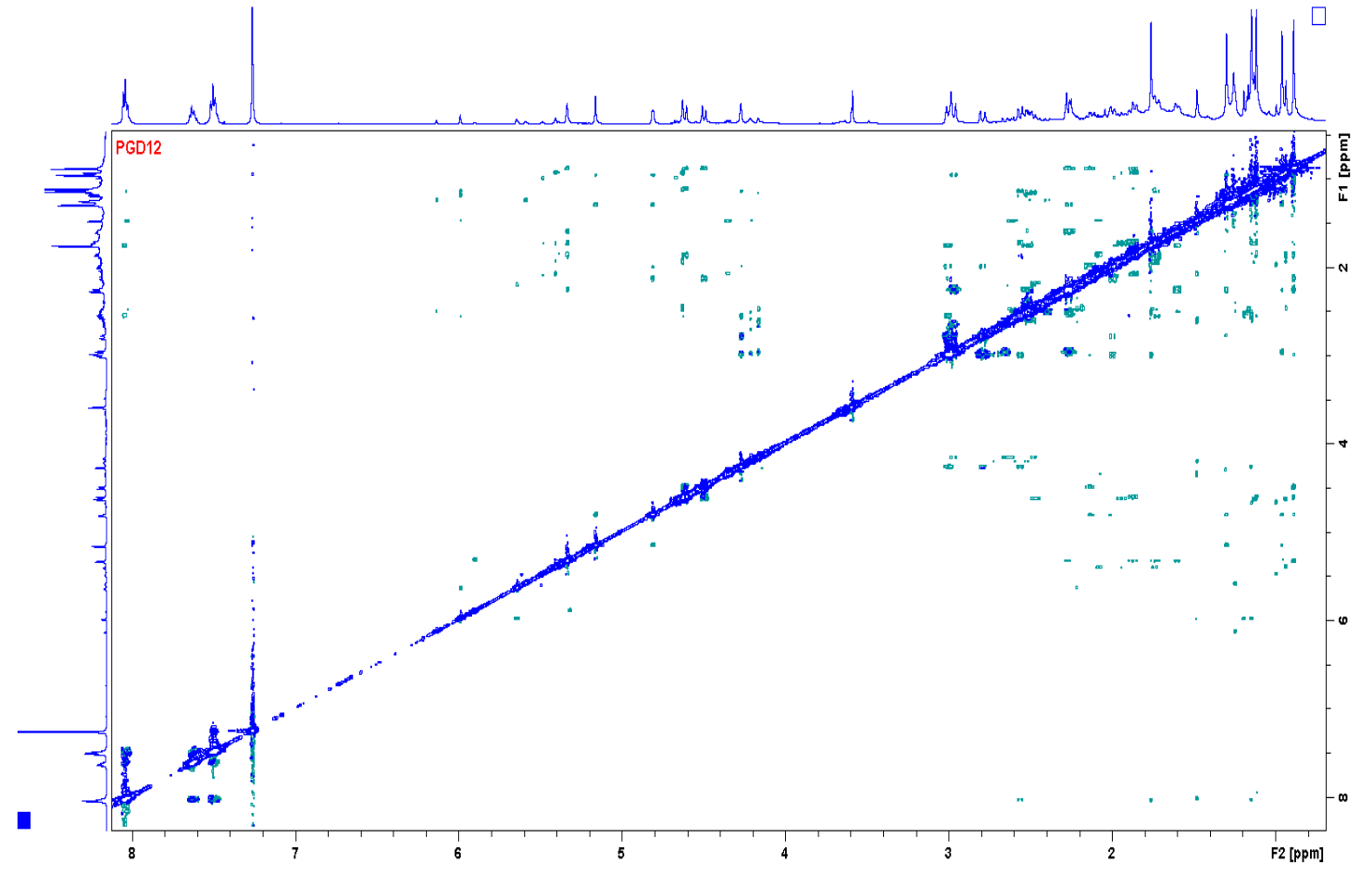


Figure S101. ${ }^{1} \mathrm{H}$ NMR spectrum of picraviane $\mathrm{M}(13)$ in $\mathrm{CDCl}_{3}(600 \mathrm{MHz})$.

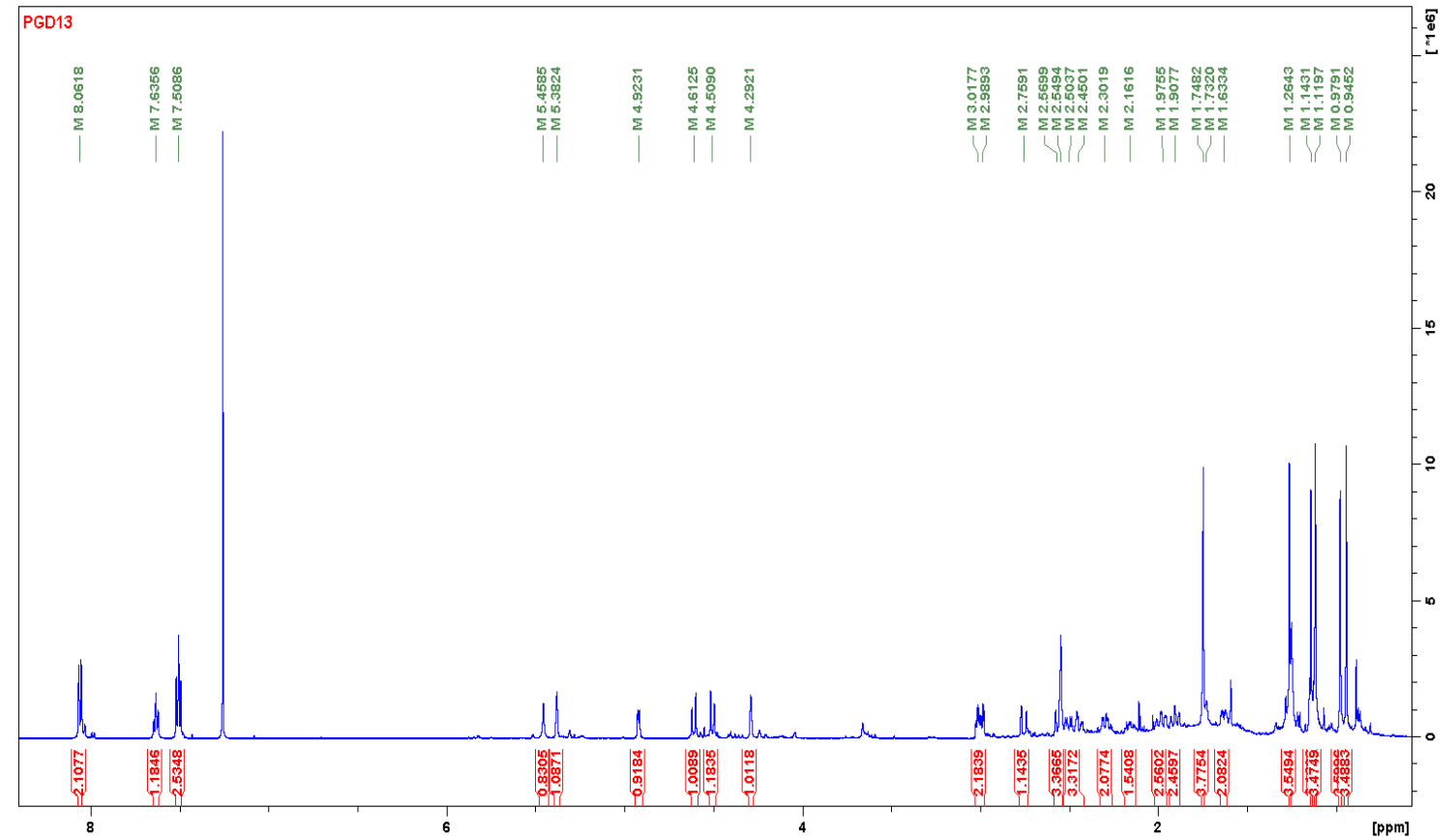

Figure S102. Expansion of the ${ }^{1} \mathrm{H}$ NMR spectrum of picraviane $\mathrm{M}(\mathbf{1 3})$ in $\mathrm{CDCl}_{3}(600 \mathrm{MHz})$.

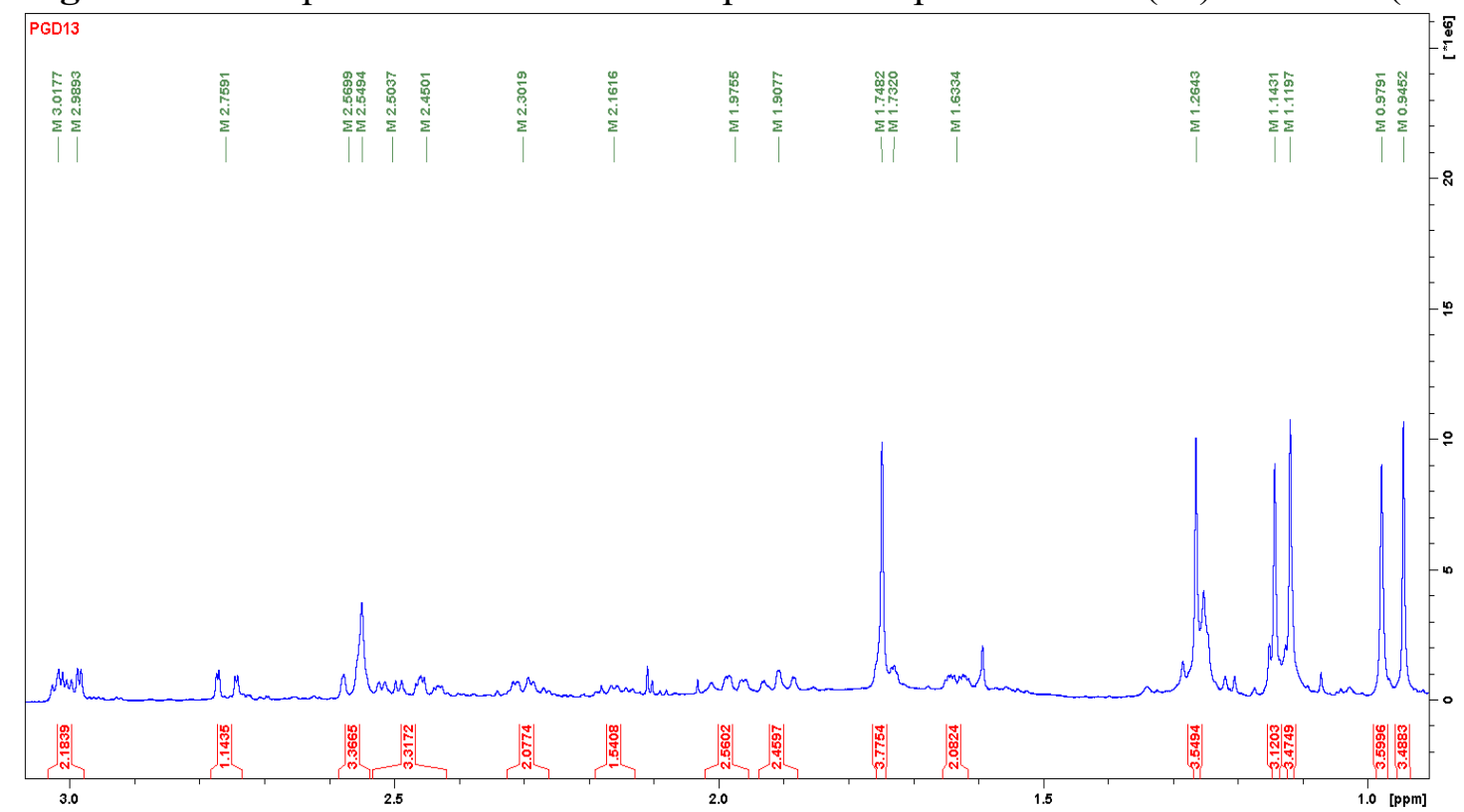


Figure S103. ${ }^{13} \mathrm{C}$ NMR spectrum of picraviane $\mathrm{M}(\mathbf{1 3})$ in $\mathrm{CDCl}_{3}(150 \mathrm{MHz})$.

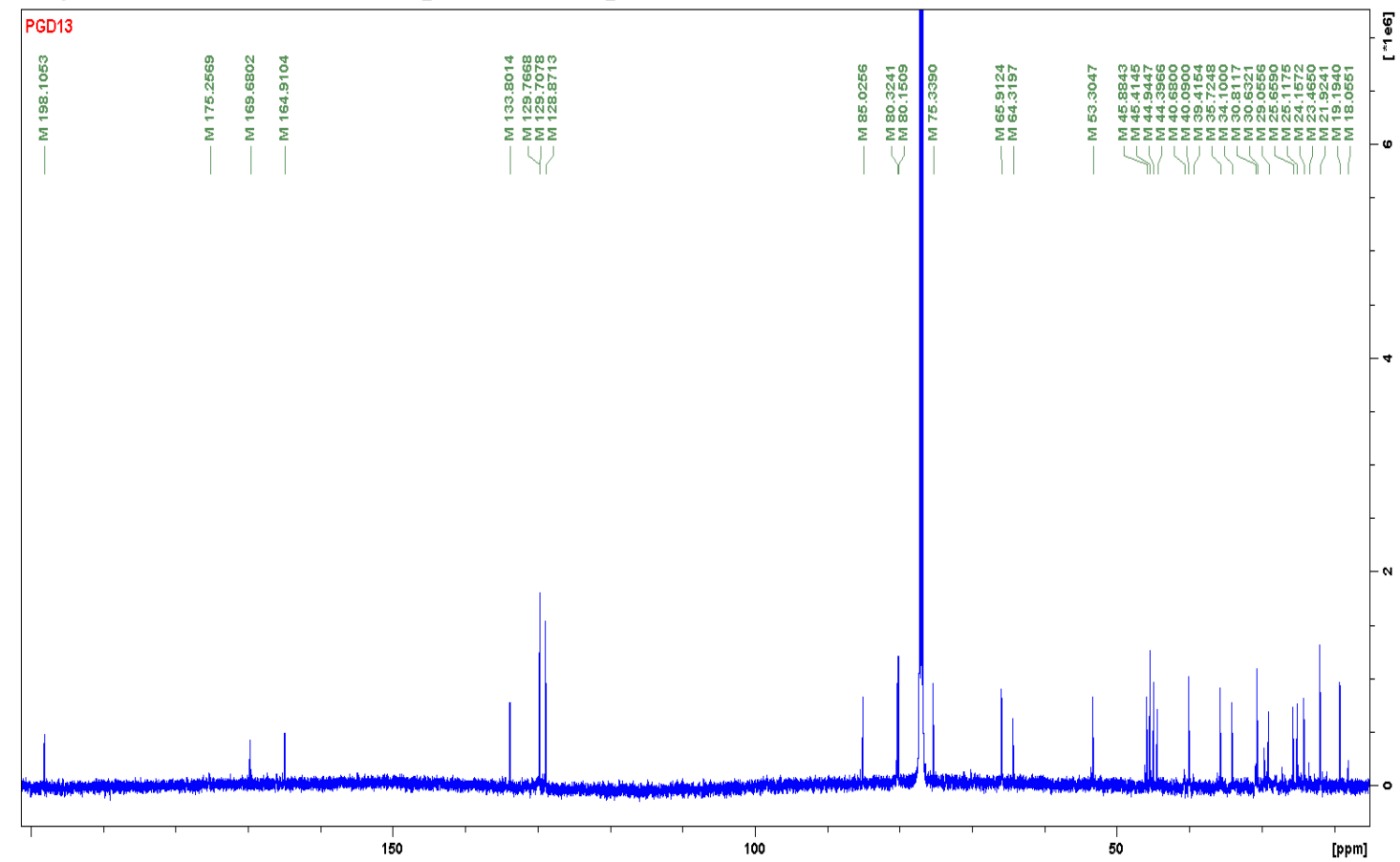

Figure S104. HSQC spectrum of picraviane $\mathrm{M}(13)$ in $\mathrm{CDCl}_{3}(600 \mathrm{MHz})$.

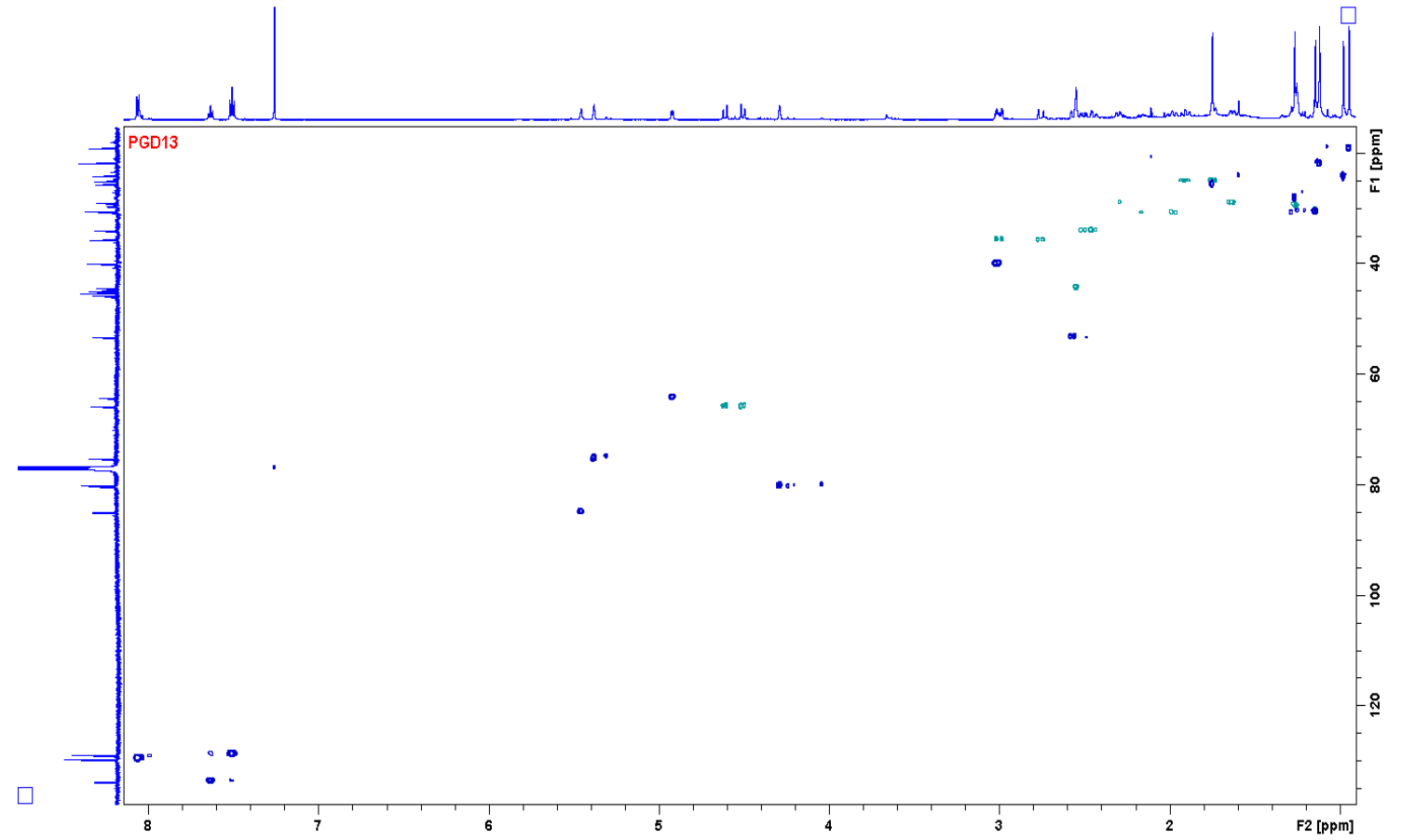


Figure S105. HMBC spectrum of picraviane $\mathrm{M}(13)$ in $\mathrm{CDCl}_{3}(600 \mathrm{MHz})$.

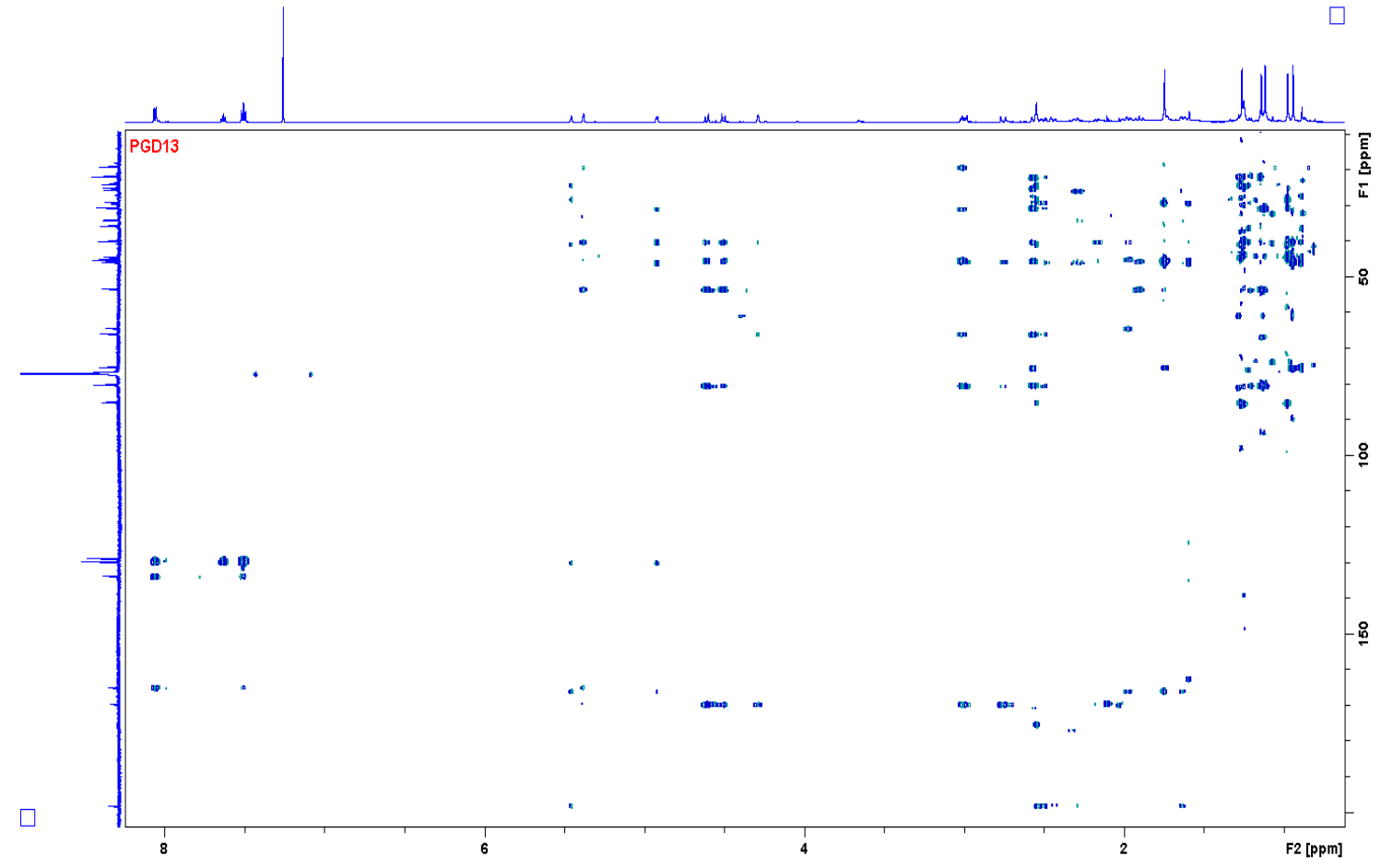

Figure S106. COSY spectrum of picraviane $\mathrm{M}(13)$ in $\mathrm{CDCl}_{3}(600 \mathrm{MHz})$.

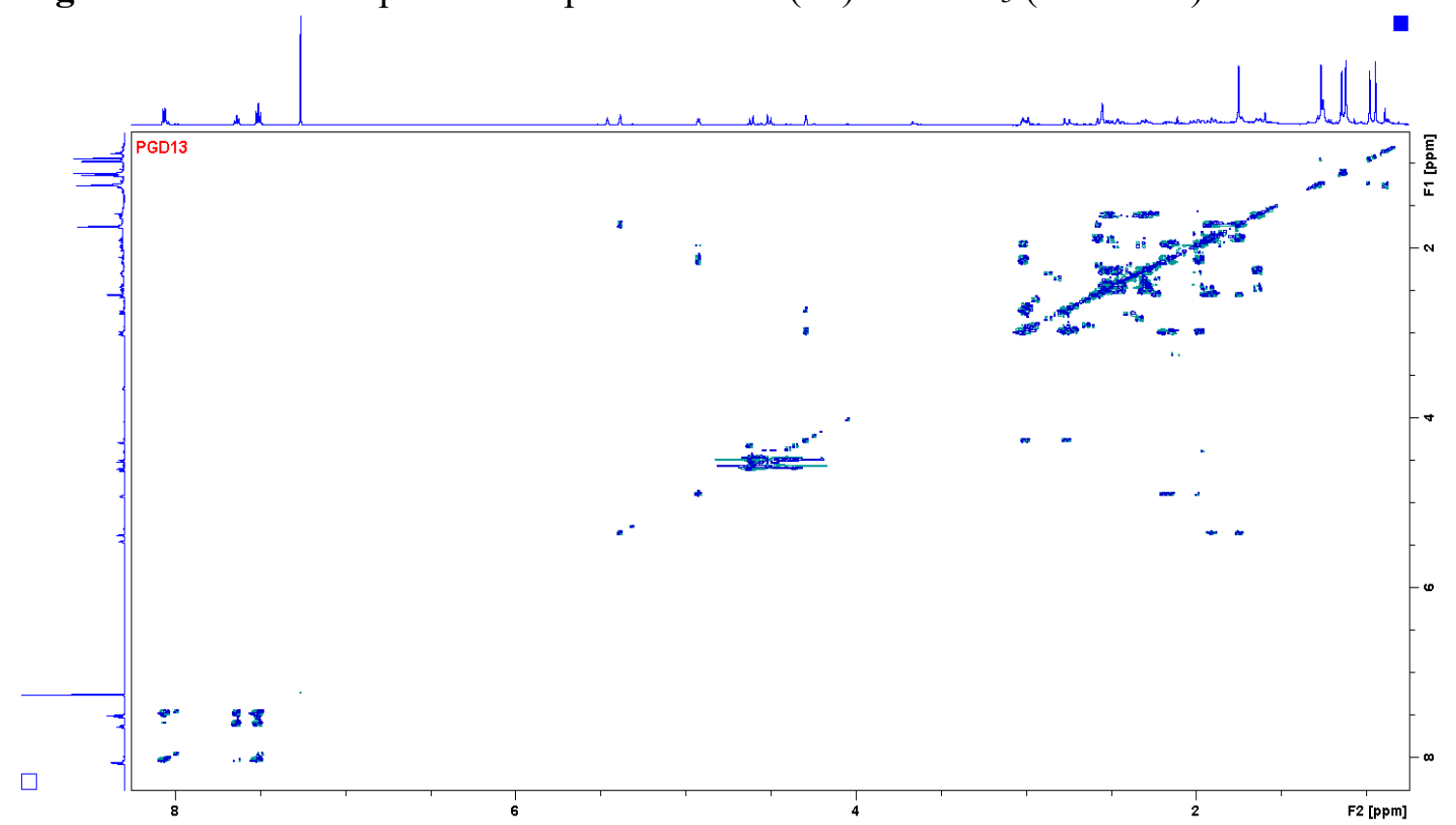


Figure S107. NOESY spectrum of picraviane $\mathrm{M}(13)$ in $\mathrm{CDCl}_{3}(600 \mathrm{MHz})$.

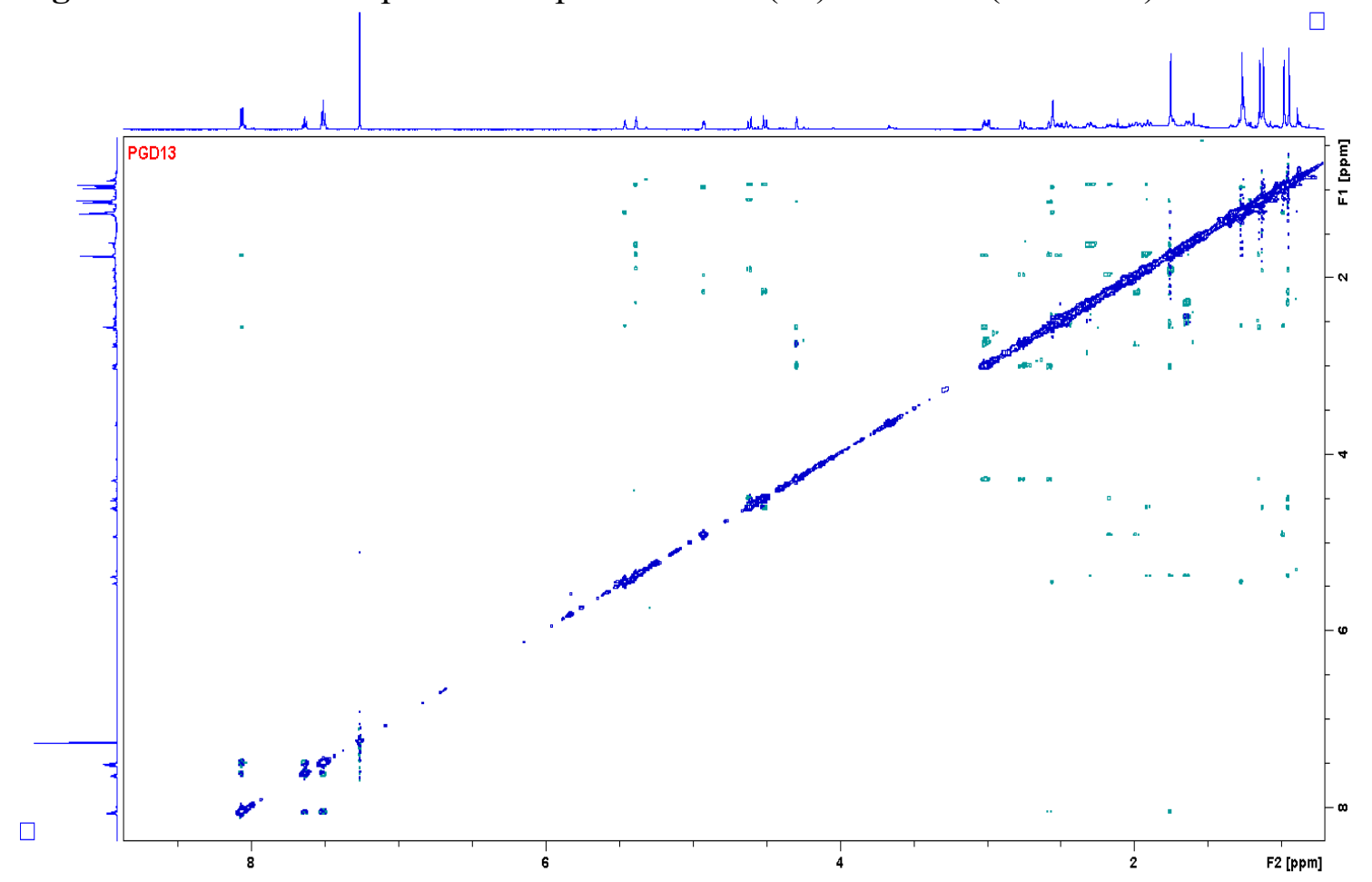

Figure S108. ${ }^{1} \mathrm{H}$ NMR spectrum of picraviane $\mathrm{H}(7)$ in $\mathrm{CDCl}_{3}(600 \mathrm{MHz})$.

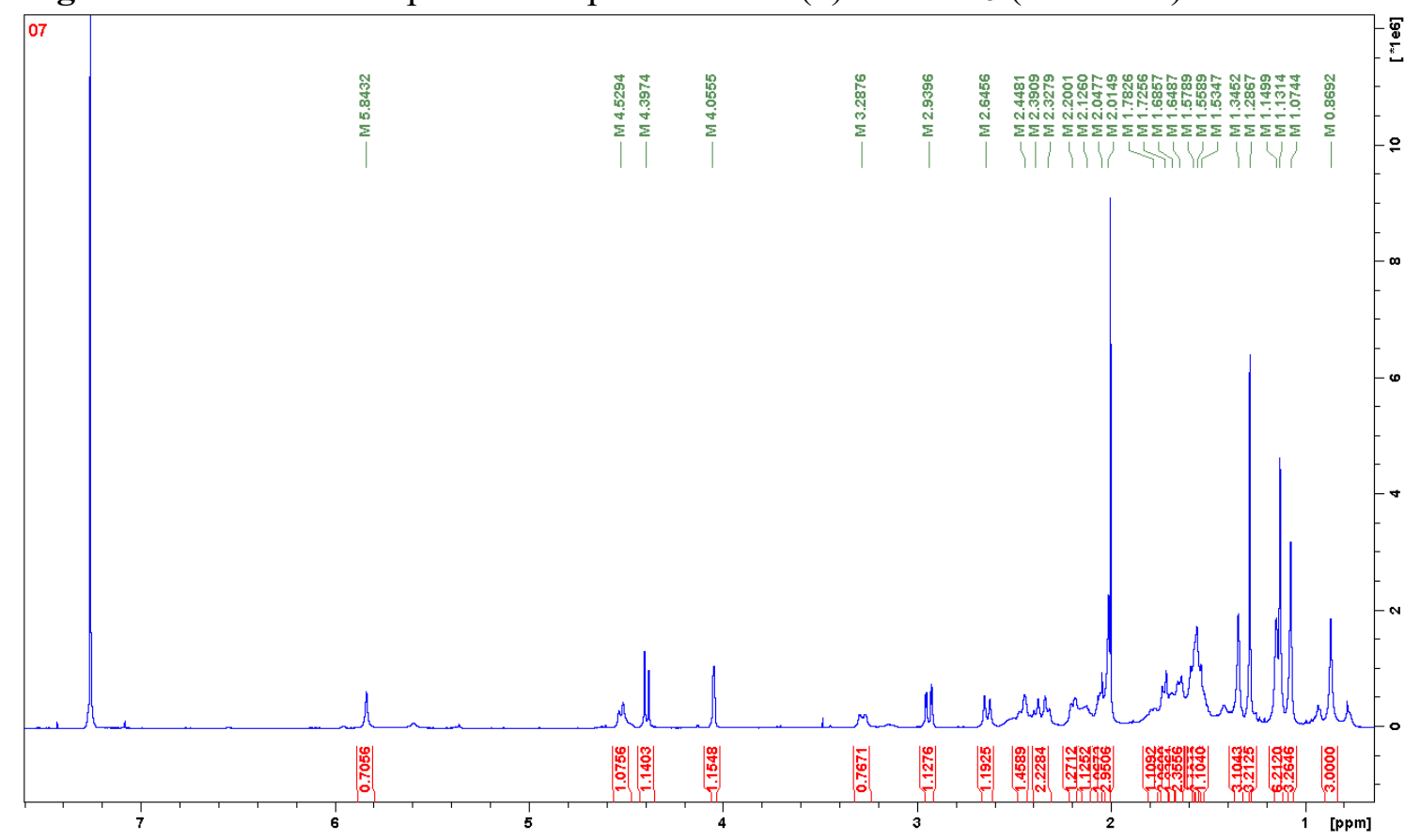


Figure S109. Expansion of the ${ }^{1} \mathrm{H}$ NMR spectrum of picraviane $\mathrm{H}(7)$ in $\mathrm{CDCl}_{3}(600 \mathrm{MHz})$.

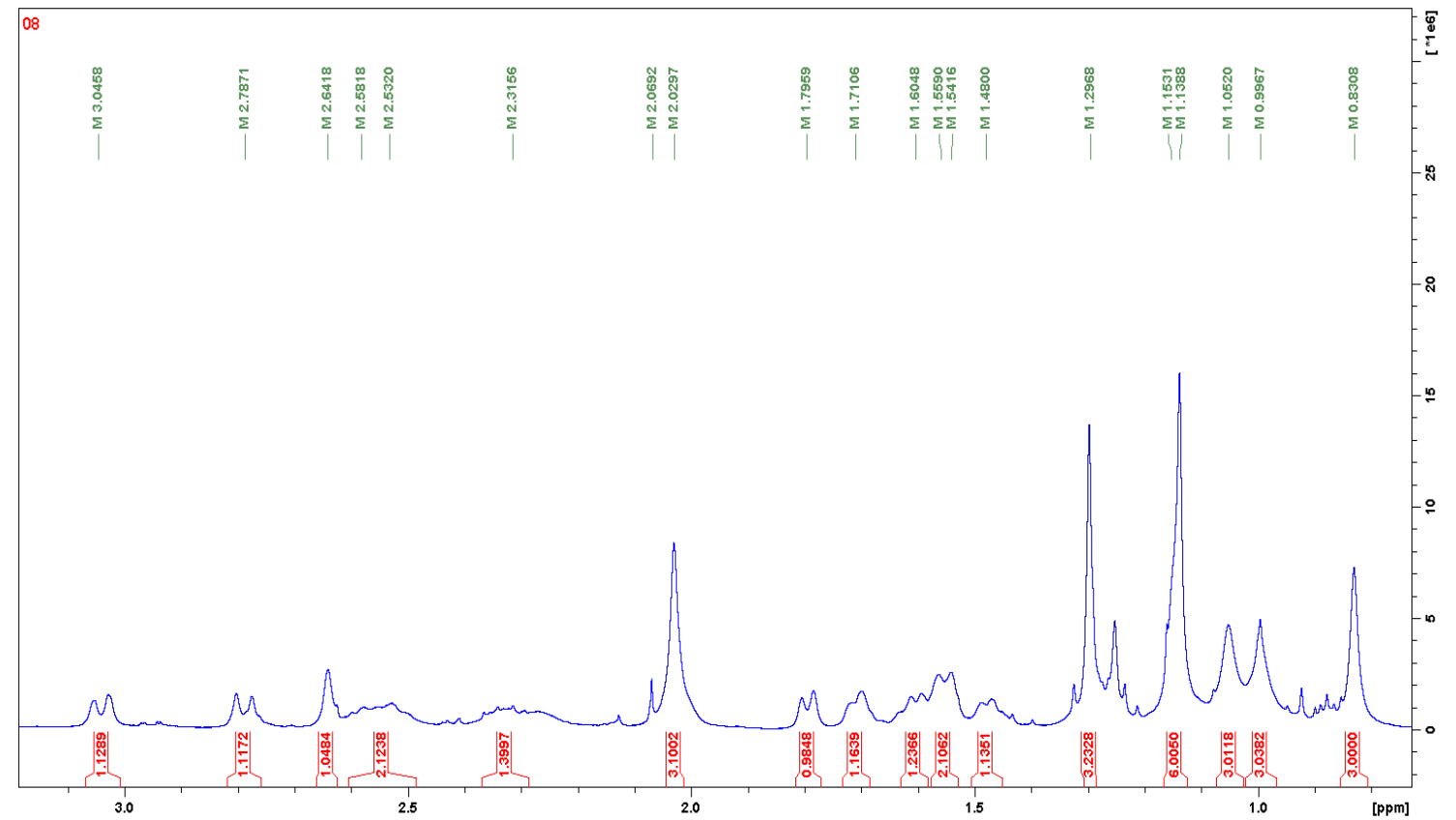

Figure S110. ${ }^{13} \mathrm{C}$ NMR spectrum of picraviane $\mathrm{H}(7)$ in $\mathrm{CDCl}_{3}(150 \mathrm{MHz})$.

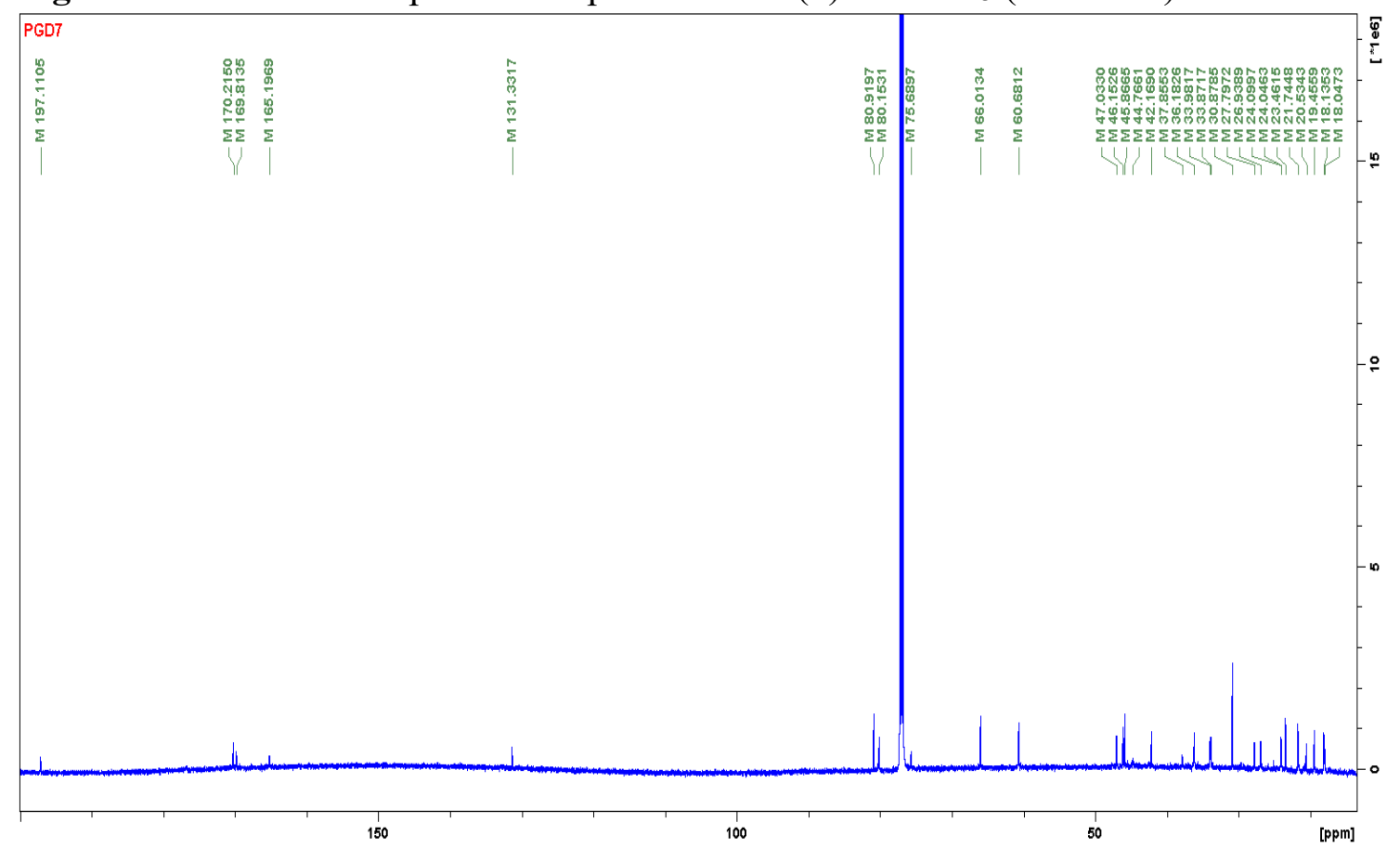


Figure S111. HSQC spectrum of picraviane $\mathrm{H}(7)$ in $\mathrm{CDCl}_{3}(600 \mathrm{MHz})$.

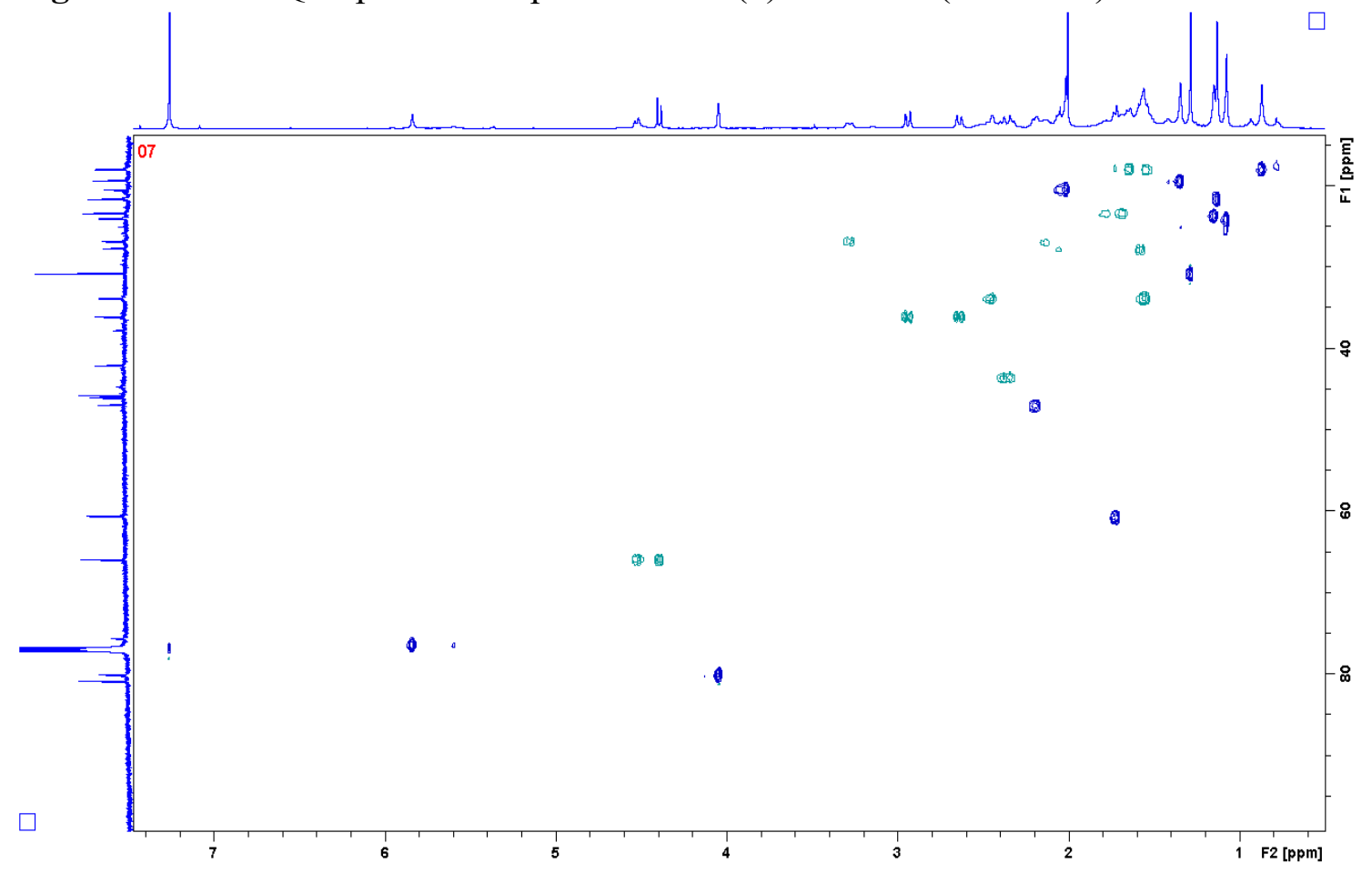

Figure S112. HMBC spectrum of picraviane $\mathrm{H}(7)$ in $\mathrm{CDCl}_{3}(600 \mathrm{MHz})$.

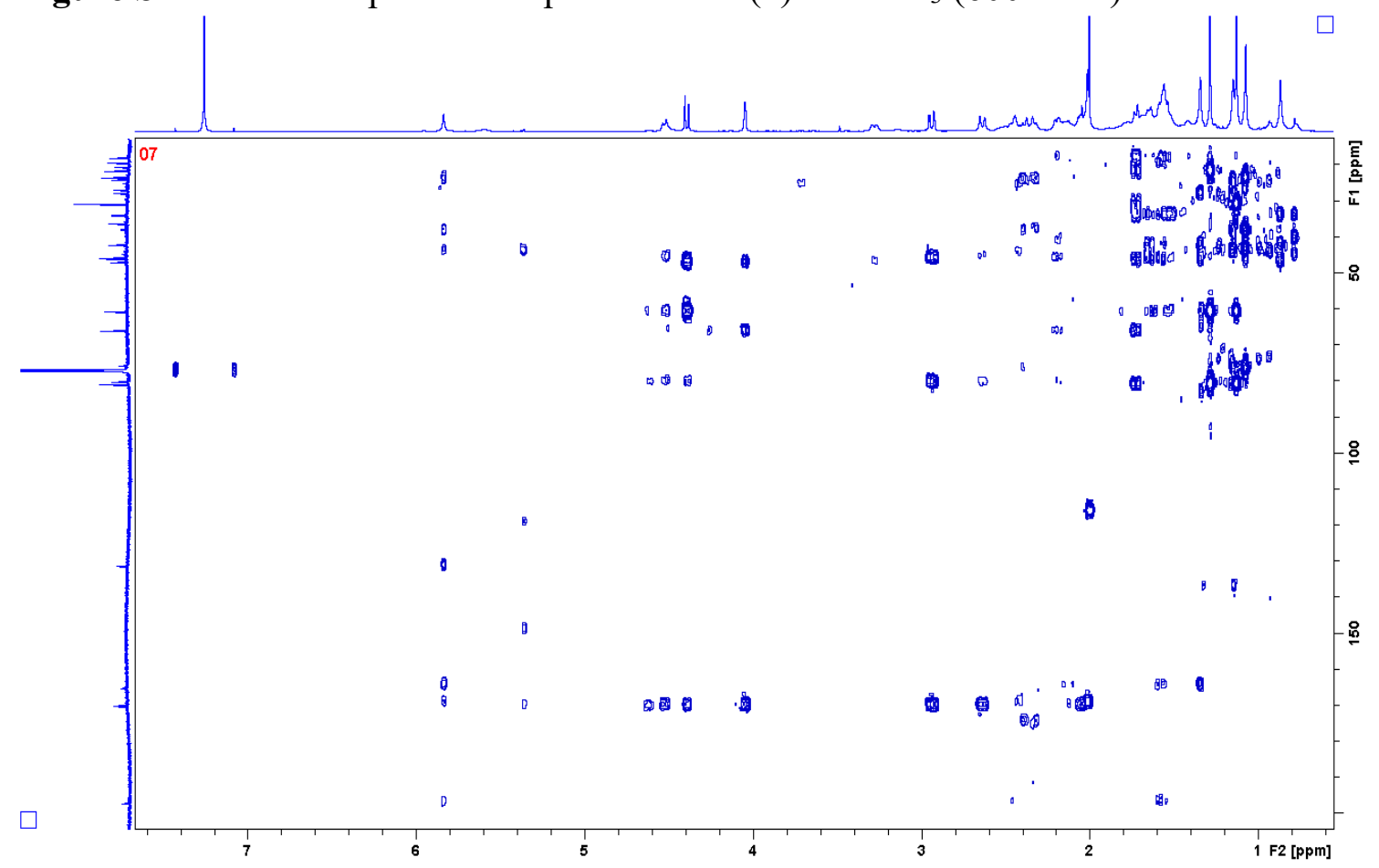


Figure S113. COSY spectrum of picraviane $\mathrm{H}(7)$ in $\mathrm{CDCl}_{3}(600 \mathrm{MHz})$.

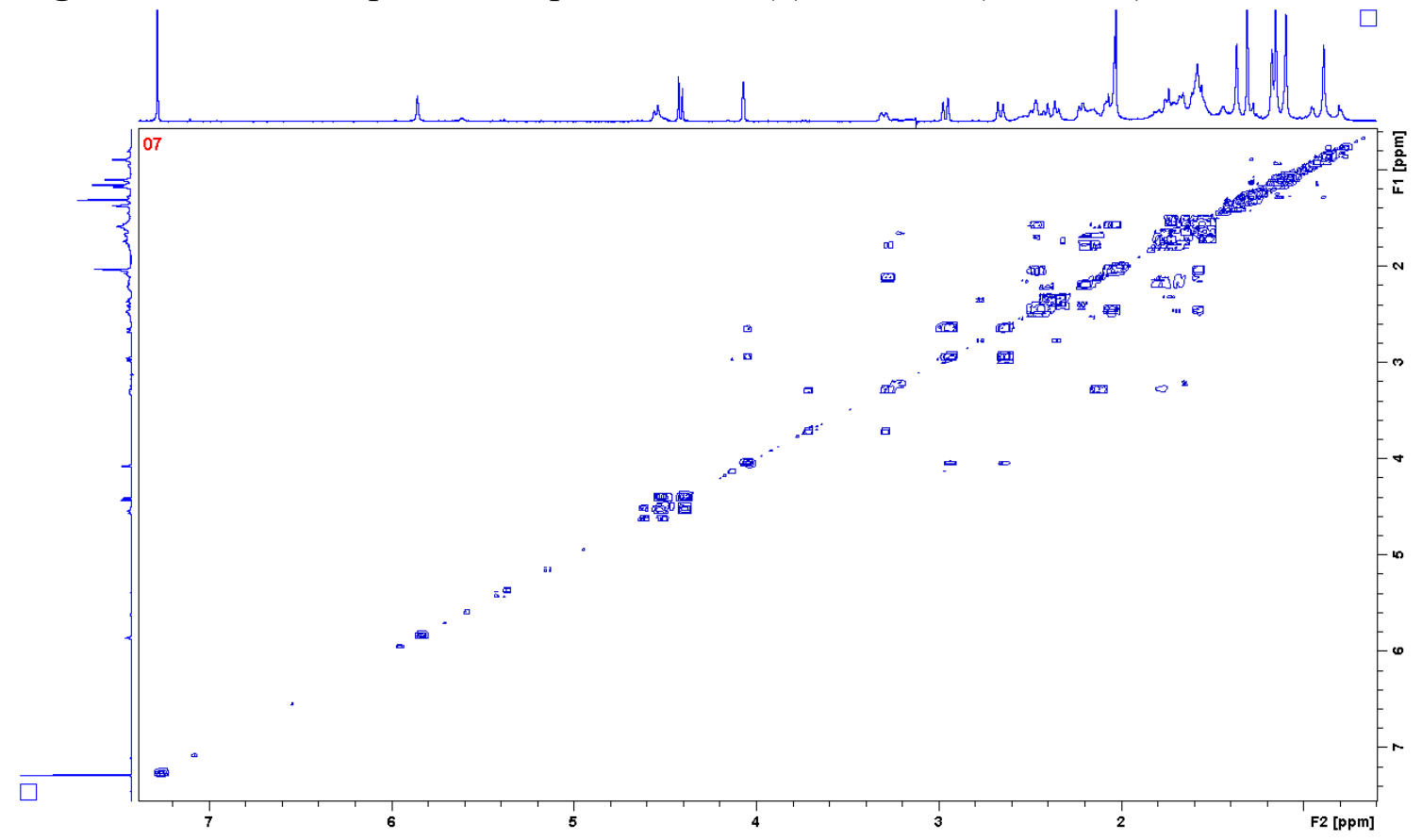

Figure S114. ROESY spectrum of picraviane $\mathrm{H}(7)$ in $\mathrm{CDCl}_{3}(600 \mathrm{MHz})$.

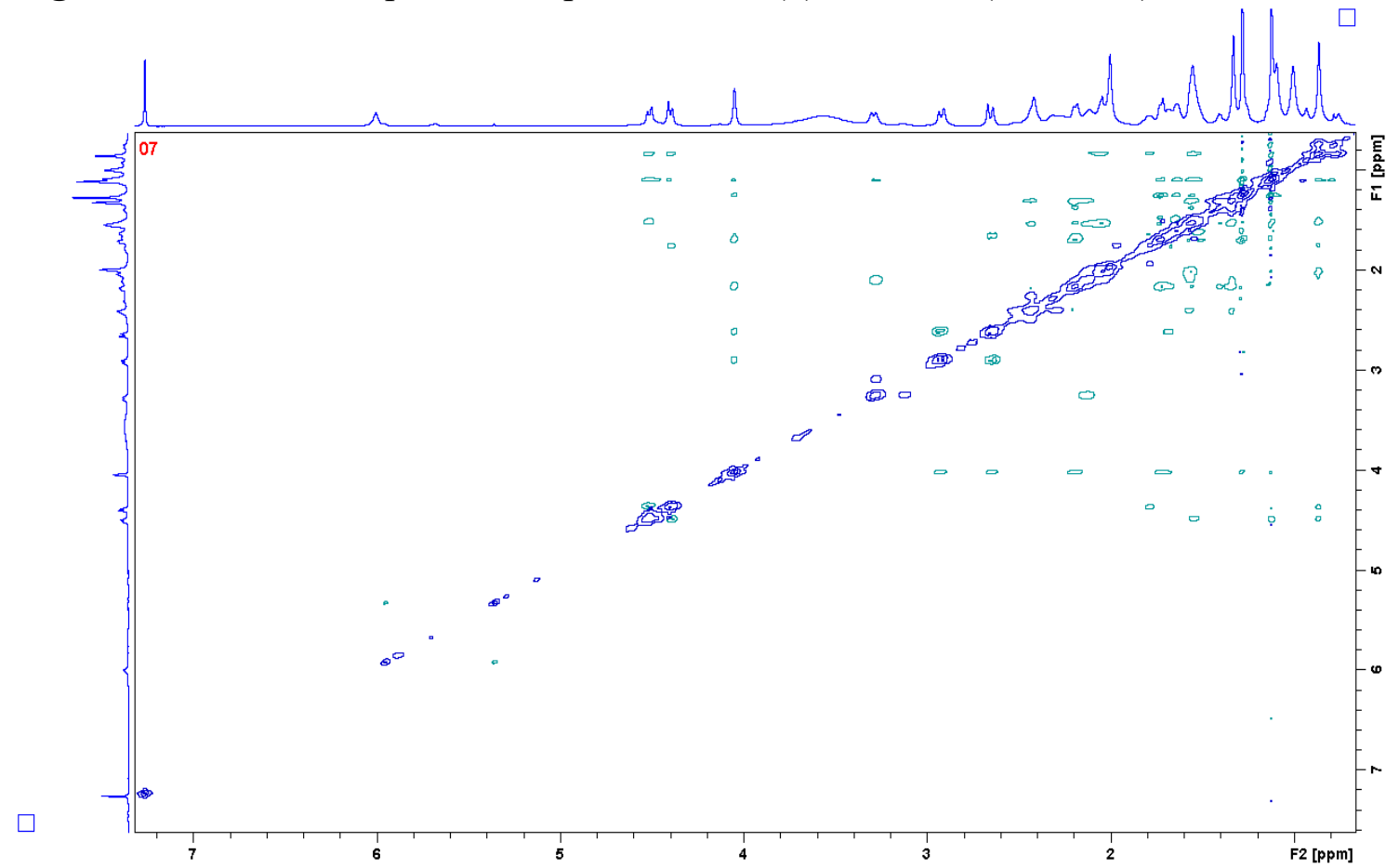


Figure S115. ${ }^{1} \mathrm{H}$ NMR spectrum of picraviane $\mathrm{N}(14)$ in $\mathrm{CDCl}_{3}(600 \mathrm{MHz})$.

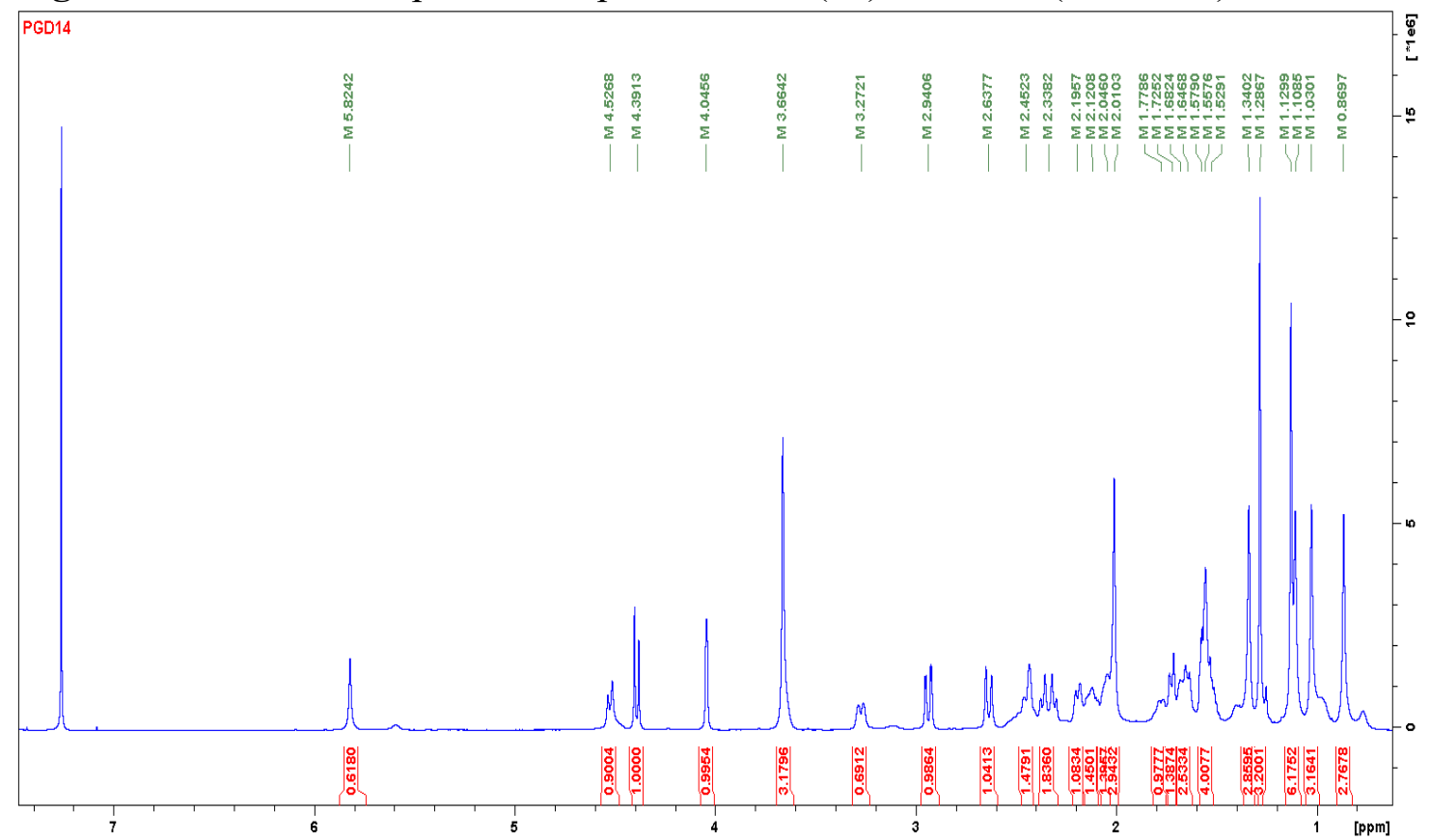

Figure S116. Expansion of the ${ }^{1} \mathrm{H}$ NMR spectrum of picraviane $\mathrm{N}(14)$ in $\mathrm{CDCl}_{3}(600 \mathrm{MHz})$.

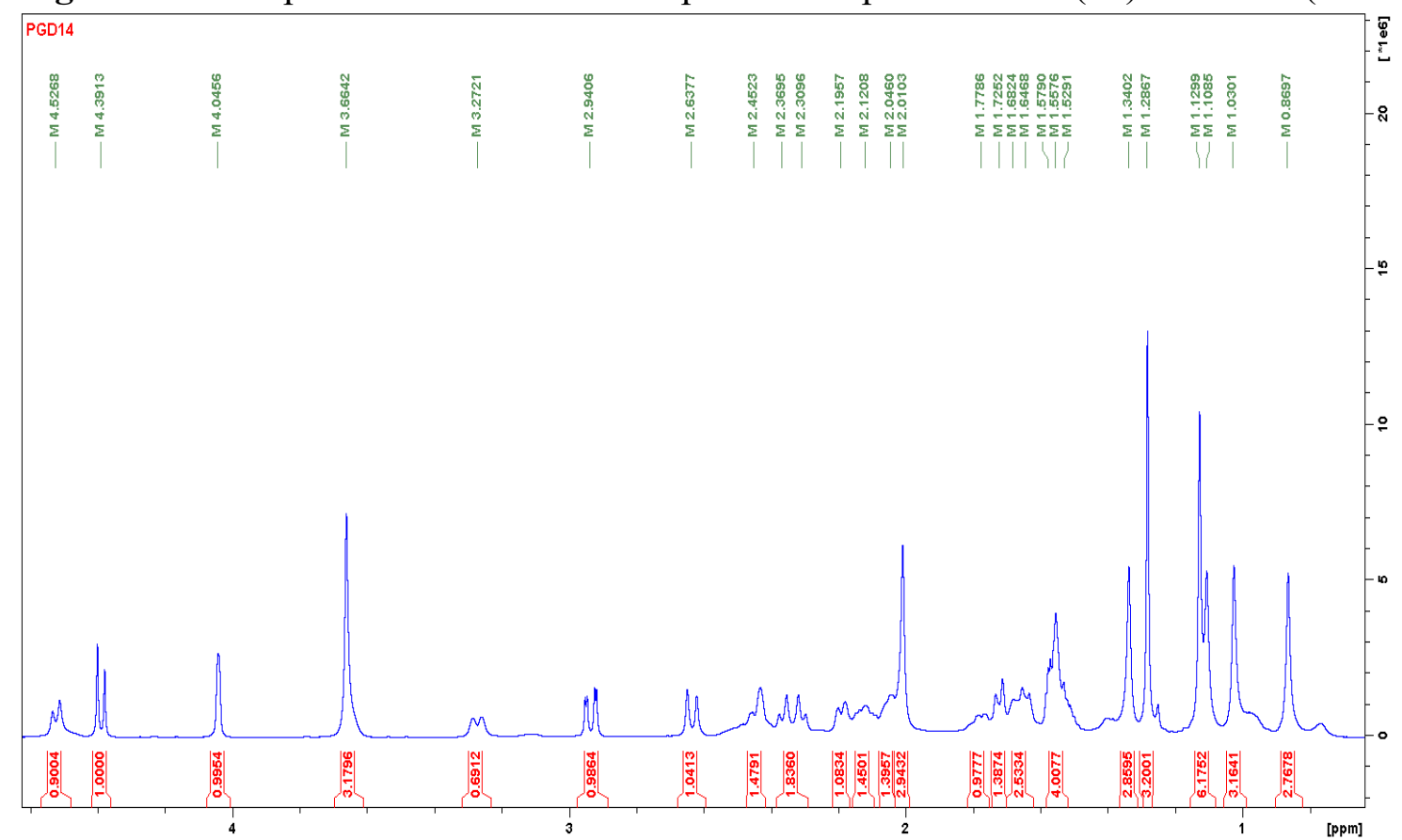


Figure S117. ${ }^{13} \mathrm{C}$ NMR spectrum of picraviane $\mathrm{N}(14)$ in $\mathrm{CDCl}_{3}(150 \mathrm{MHz})$.

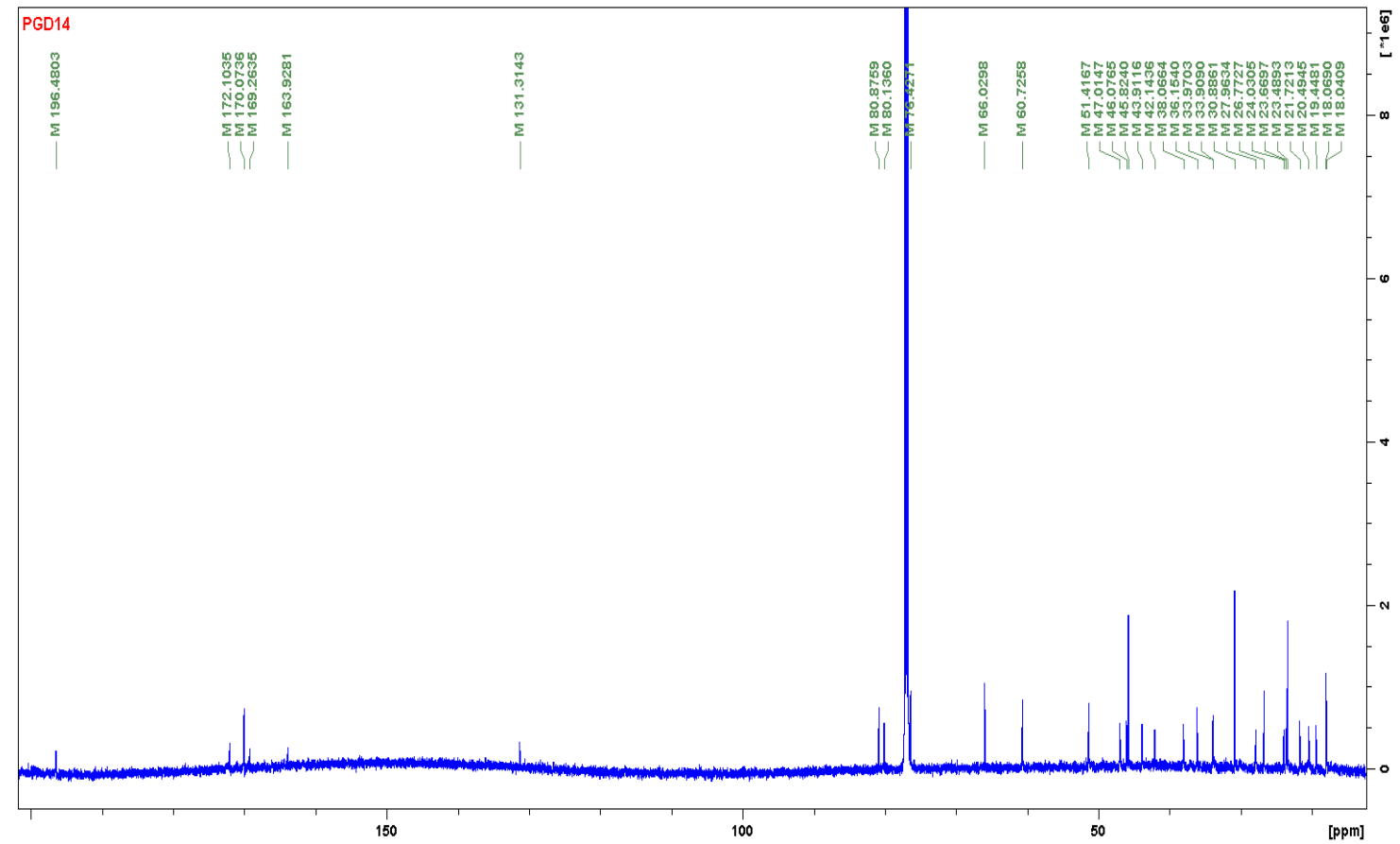

Figure S118. HSQC spectrum of picraviane $\mathrm{N}(\mathbf{1 4})$ in $\mathrm{CDCl}_{3}(600 \mathrm{MHz})$.

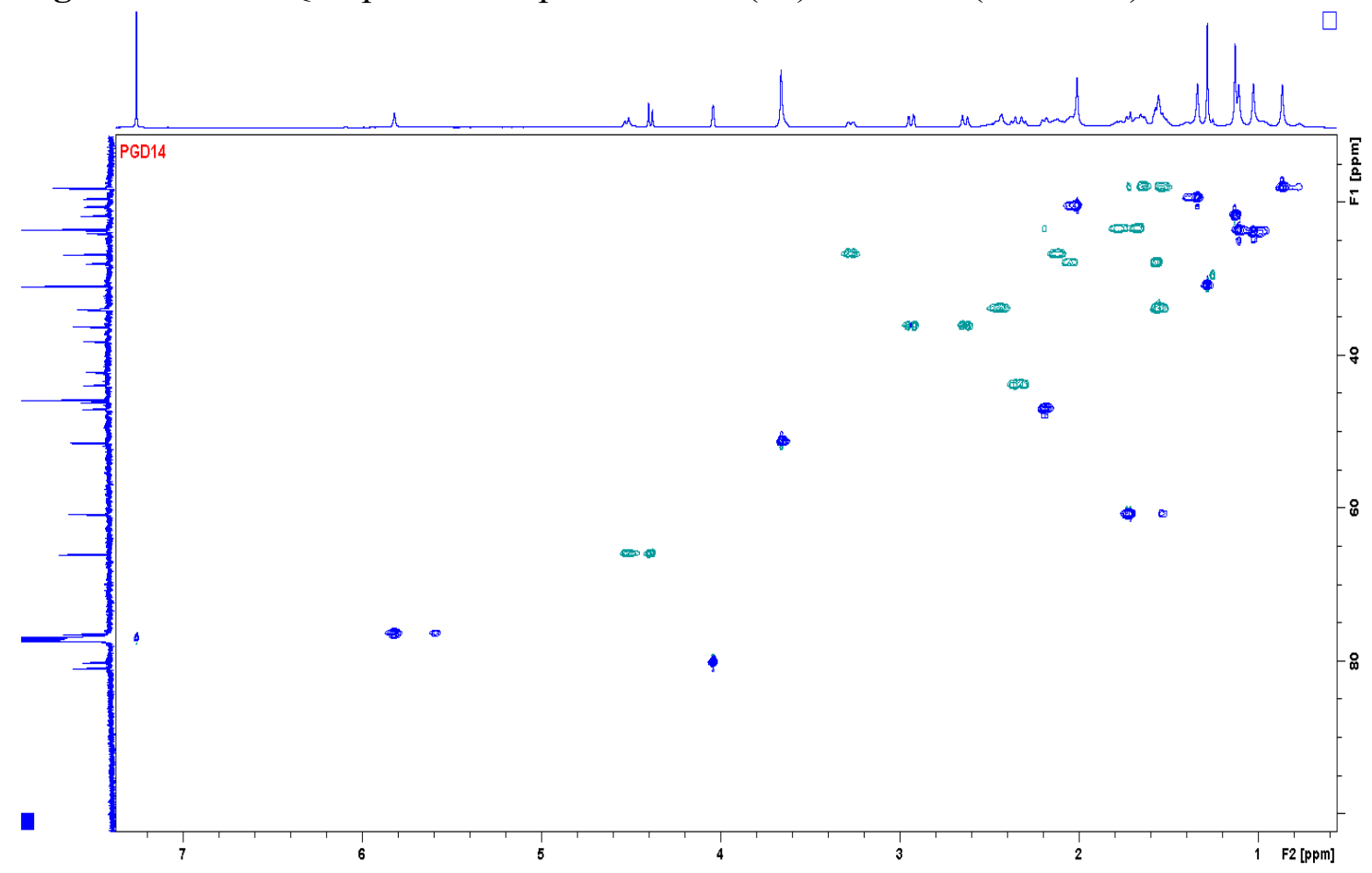


Figure S119. HMBC spectrum of picraviane $\mathrm{N}(14)$ in $\mathrm{CDCl}_{3}(600 \mathrm{MHz})$.

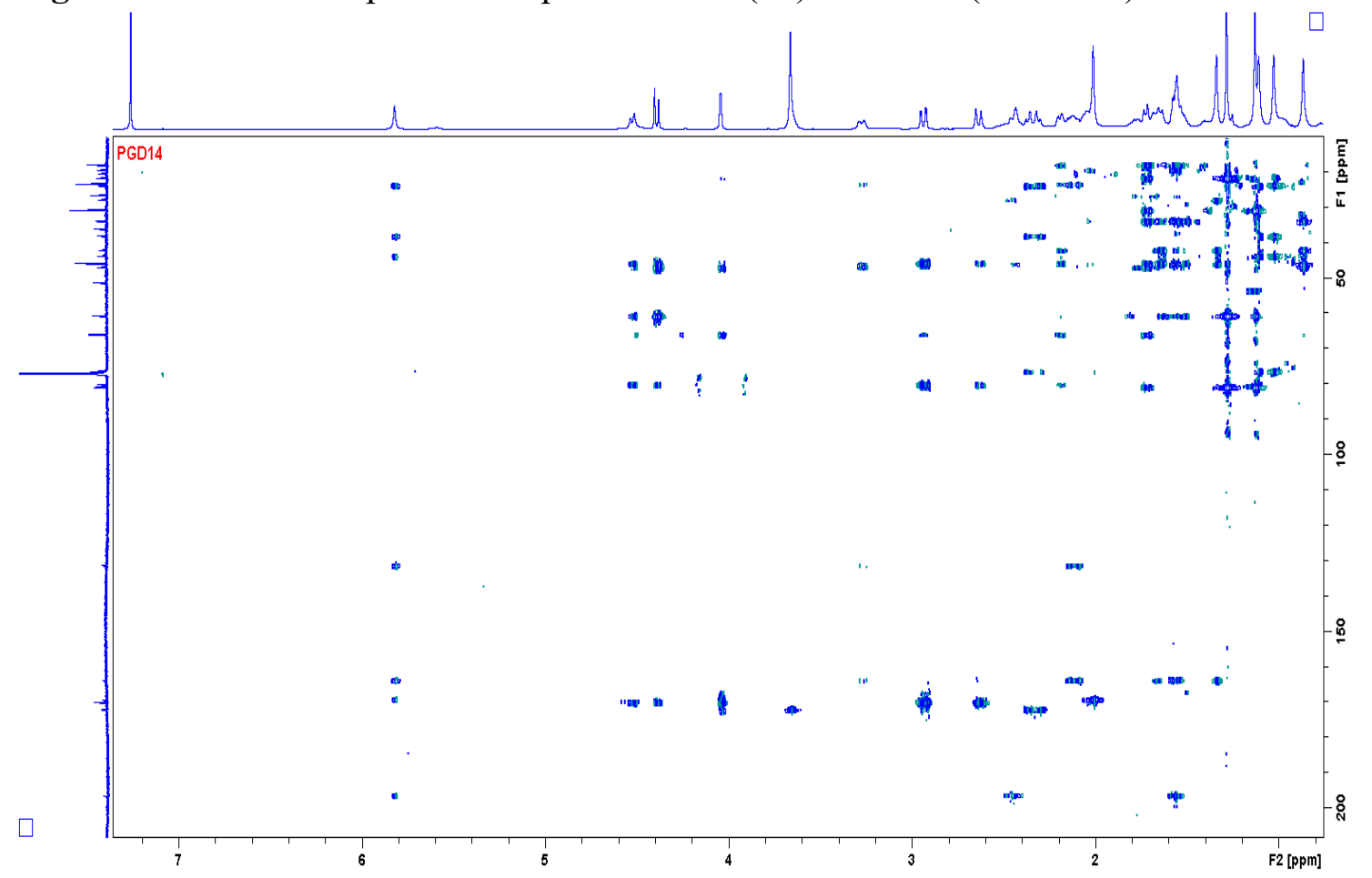

Figure S120. COSY spectrum of picraviane $\mathrm{N}(14)$ in $\mathrm{CDCl}_{3}(600 \mathrm{MHz})$.

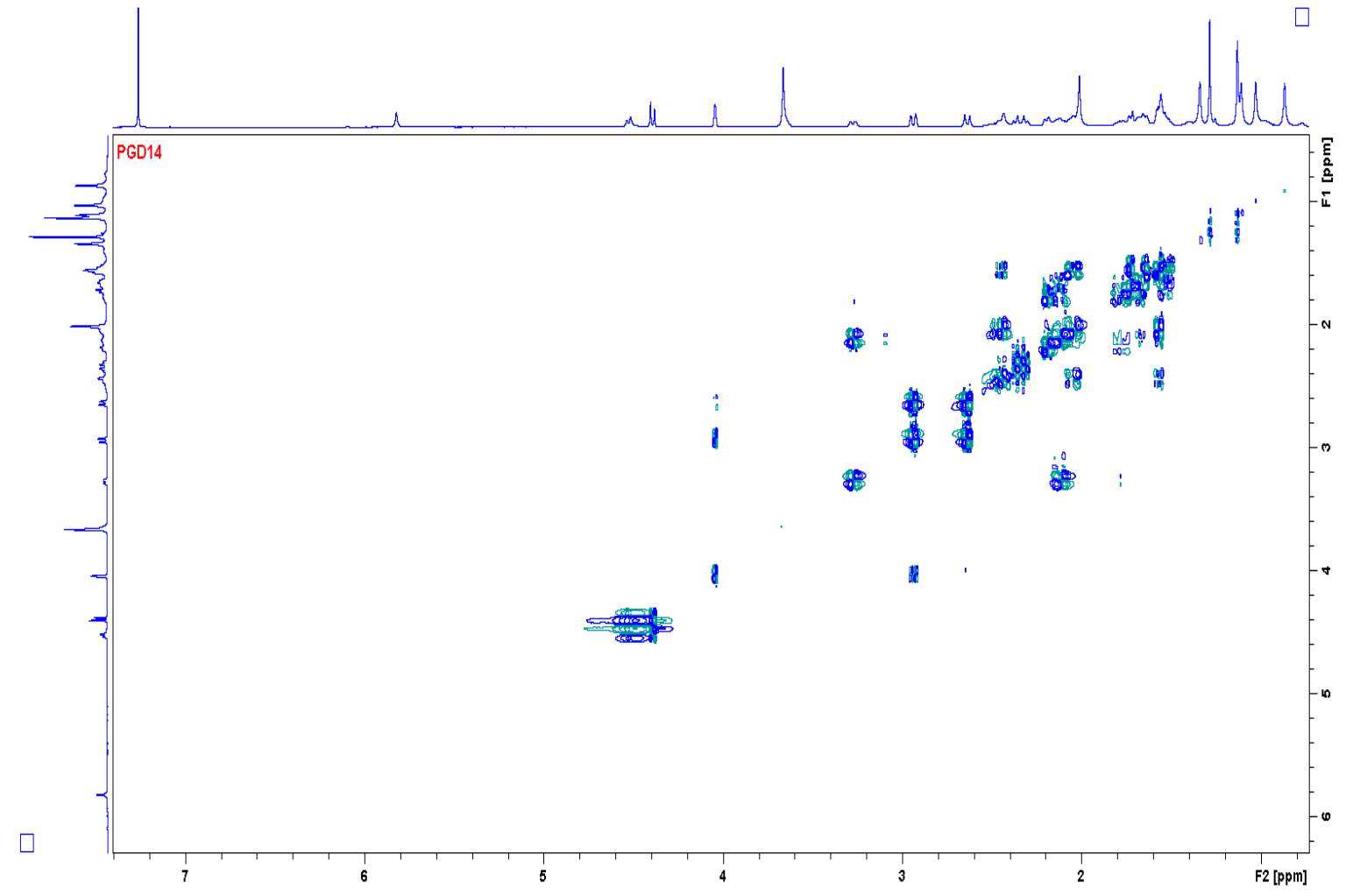


Figure S121. NOESY spectrum of picraviane N (14) in $\mathrm{CDCl}_{3}(600 \mathrm{MHz})$.

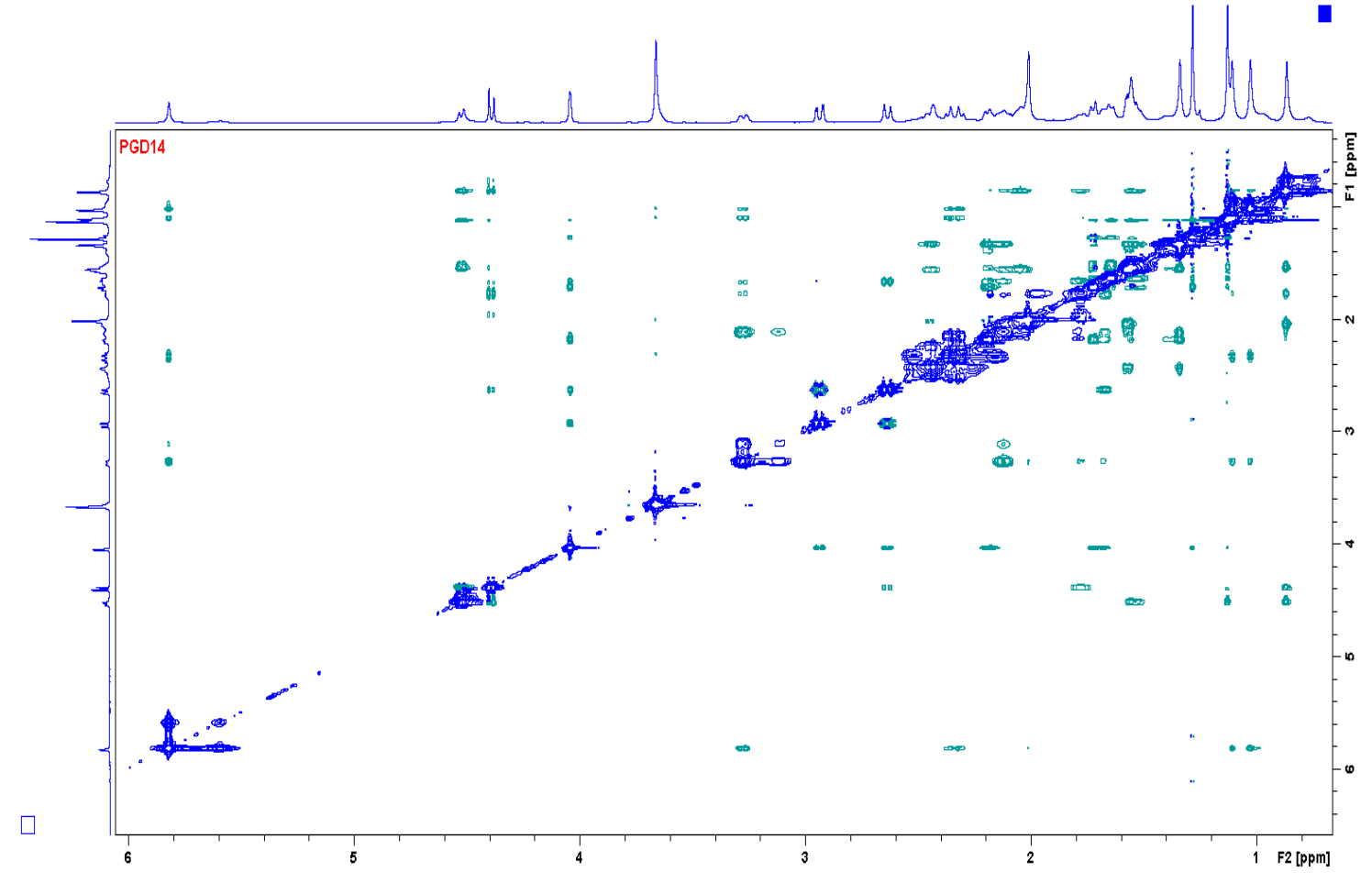


Figure S122. Structures of the five lowest-energy conformers of $(1 S, 5 R, 8 R, 9 S, 10 R, 14 S, 19 S)$ 7 at the B3LYP/PCM(MeOH)/TZVP level.

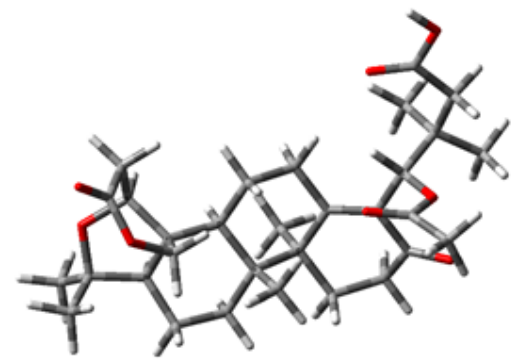

Conf. $1,0.0 \mathrm{kcal}^{-1}(30 \%)$

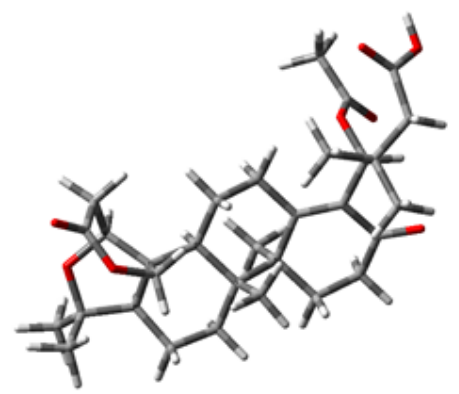

Conf. $3,+0.3 \mathrm{kcal}^{-1}(17 \%)$

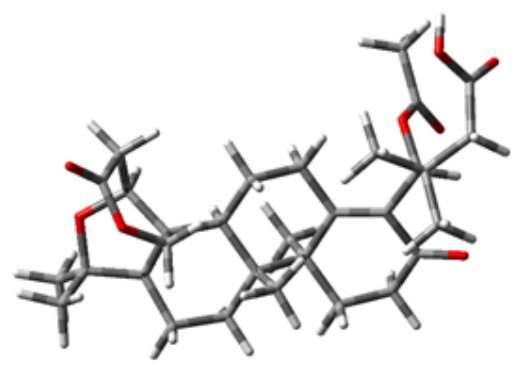

Conf. $2,+0.1 \mathrm{kcal}^{-1}(27 \%)$

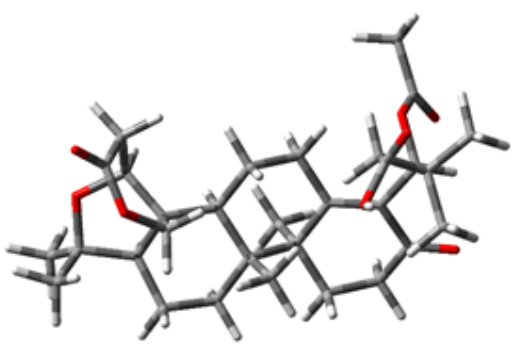

Conf. $4,+0.4 \mathrm{kcal}^{-1}(15 \%)$

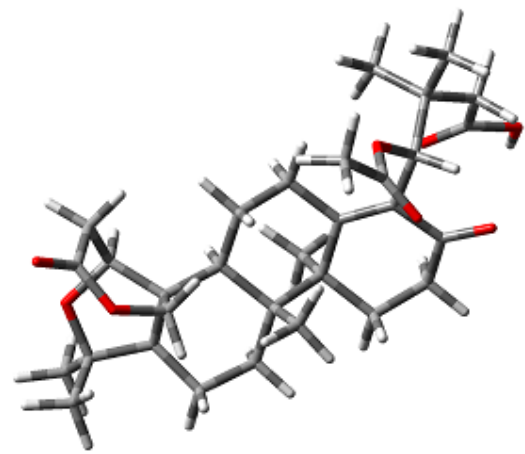

Conf. $5,+1.0 \mathrm{kcal}^{-1}(05 \%)$ 
Figure S123. Comparison of the experimental and calculated UV and ECD spectra of $(1 S, 5 R, 8 R, 9 S, 10 R, 14 S, 19 S)-7$ (blue trace) and its enantiomer (red trace). Experimental spectra were acquired in methanol, and UV and ECD spectra were calculated at the [CAM$\mathrm{B} 3 \mathrm{LYP} / \mathrm{PCM}(\mathrm{MeOH}) / \mathrm{TZVP}]$ level for the Boltzmann average of the five lowest-energy conformers identified.

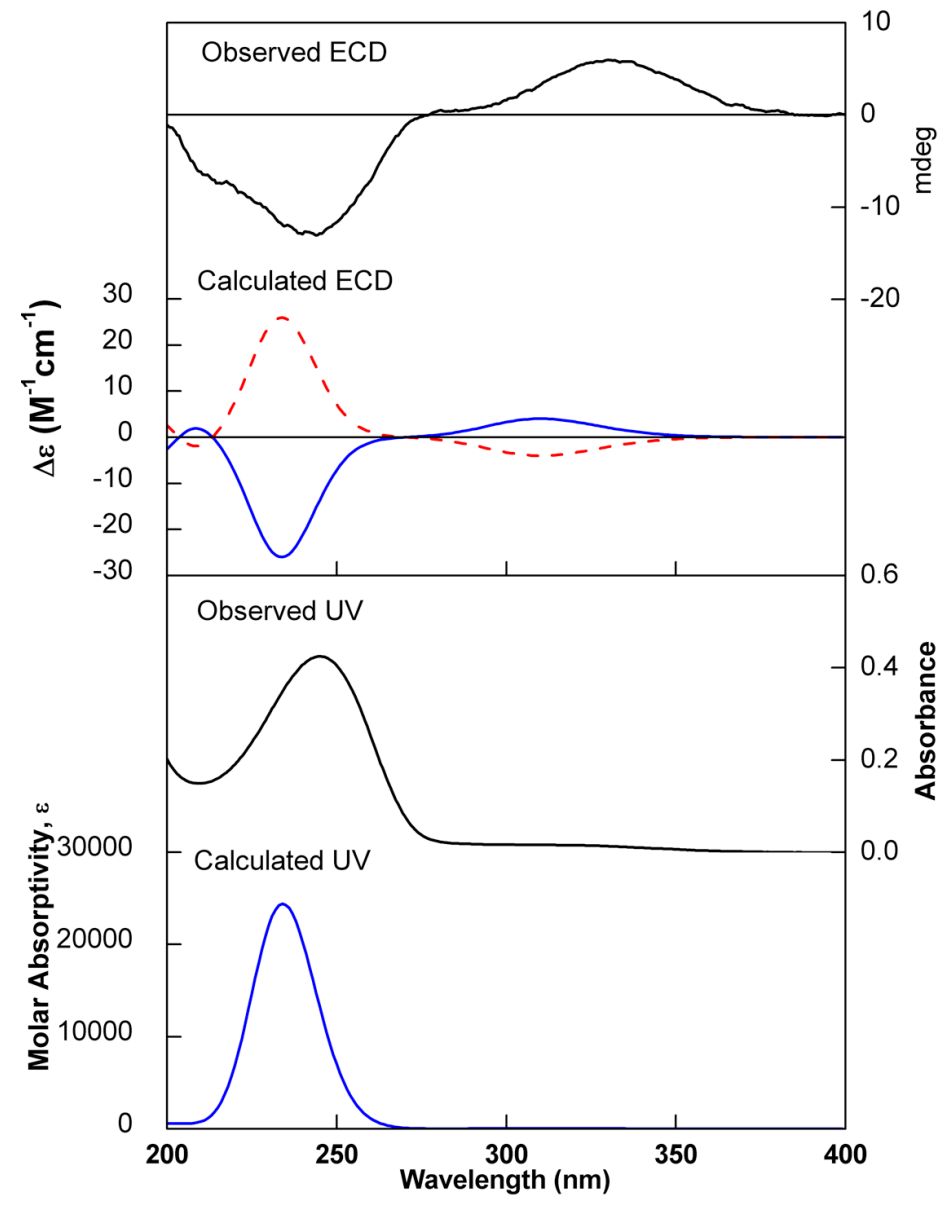


Figure S124. Structure of lowest-energy conformations and ECD spectra of the $(1 S, 5 R, 8 R, 9 S, 10 R, 14 S, 19 R)$-epimer of 7. Left: Experimental and calculated ECD spectra for the $(1 S, 5 R, 8 R, 9 S, 10 R, 14 S, 19 R)$-epimer of 7 at the CAM-B3LYP/PCM(MeOH)/TZVP level. Right: Structure, relative energies and Boltzmann populations of the lowest-energy conformers identified for $(1 S, 5 R, 8 R, 9 S, 10 R, 14 S, 19 R)-7$.

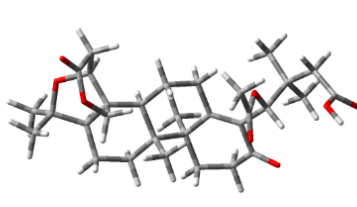

Conf. $1,0.0$ kcal $^{-1}(37 \%)$

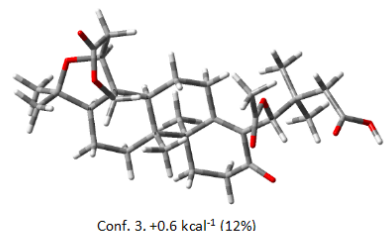

Conf. $3,+0.6 \mathrm{kcal}^{-1}(12 \%$

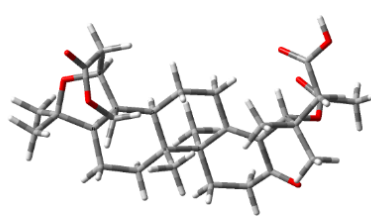

Conf. $5,+0.8 \mathrm{kcal}^{-1}(9 \%)$

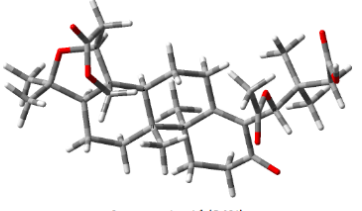

Conf. $2,+0.2 \mathrm{kcal}^{-1}(24 \%)$
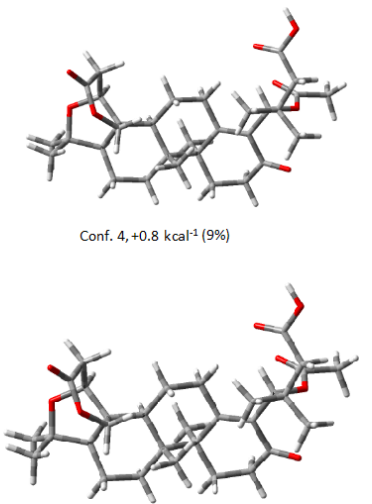

Conf. $6,+0.8 \mathrm{kcal}^{-1}(9 \%$

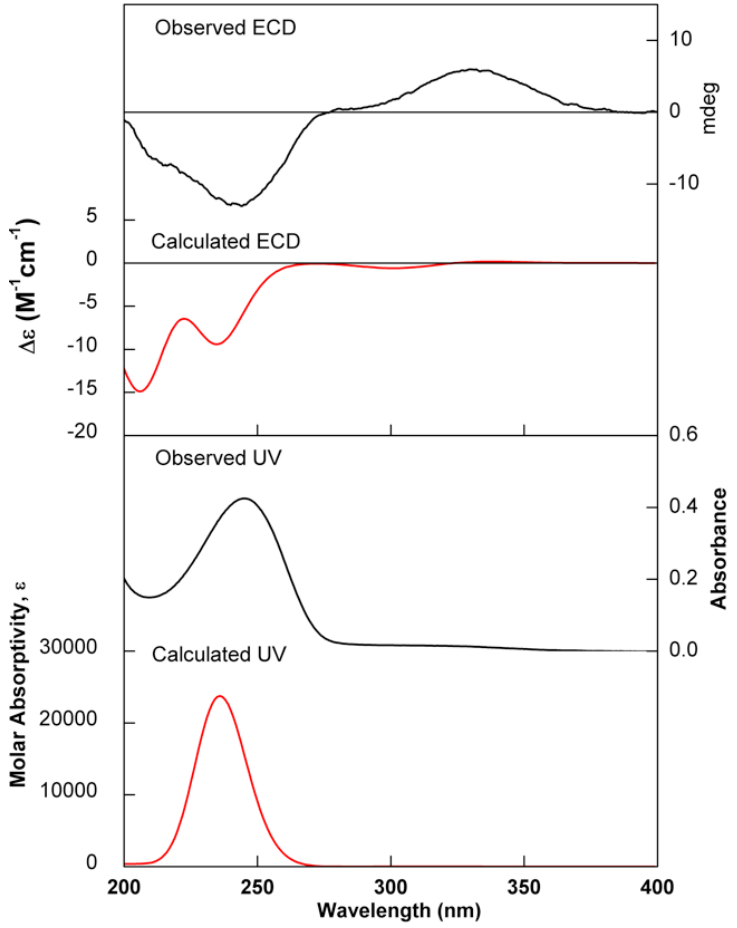


Figure S125. Comparison of the experimental ECD spectra of 7 and 14, which differ only by a methoxy group at C-22.

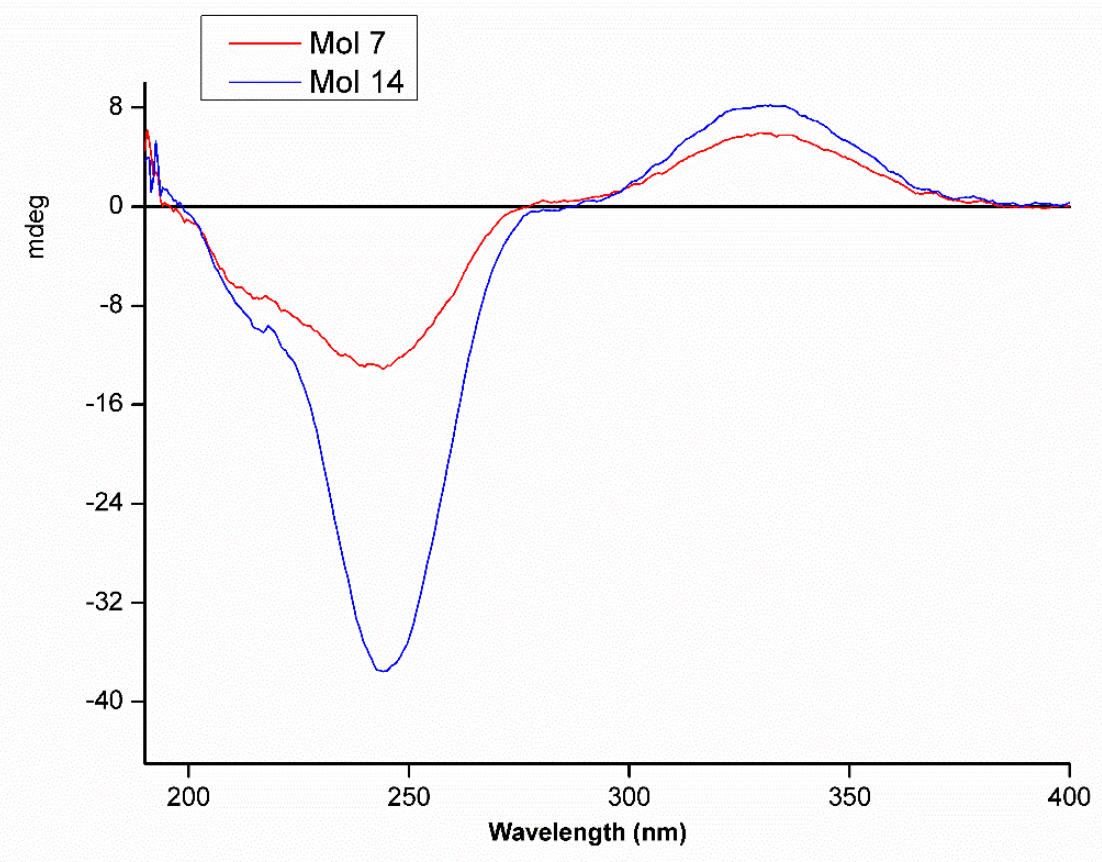

Figure S126. ${ }^{1} \mathrm{H}$ NMR spectrum of picraviane $\mathrm{O}(\mathbf{1 5})$ in $\mathrm{CDCl}_{3}(600 \mathrm{MHz})$.

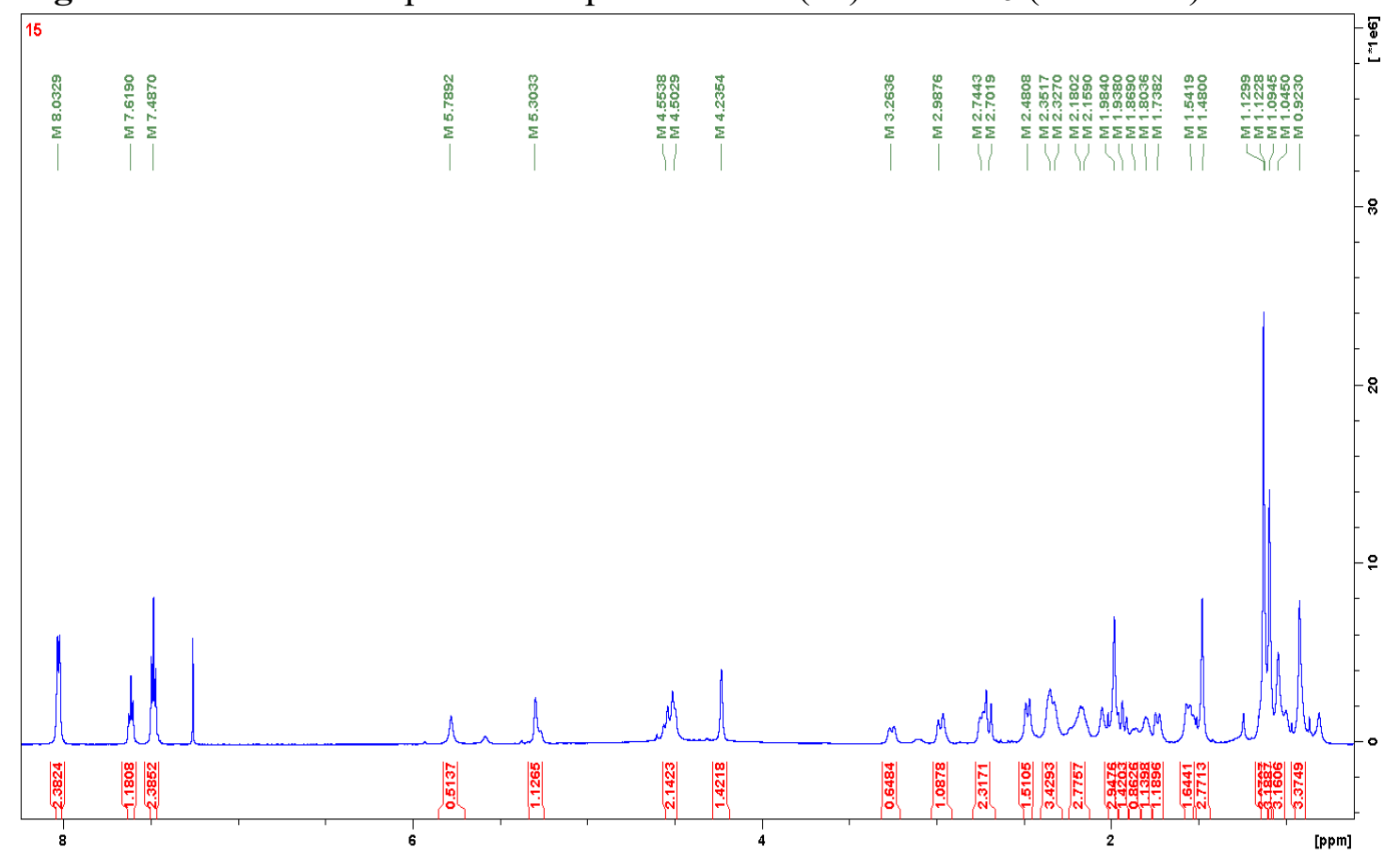


Figure S127. Expansion of the ${ }^{1} \mathrm{H}$ NMR spectrum of picraviane $\mathrm{O}(15)$ in $\mathrm{CDCl}_{3}(600 \mathrm{MHz})$.

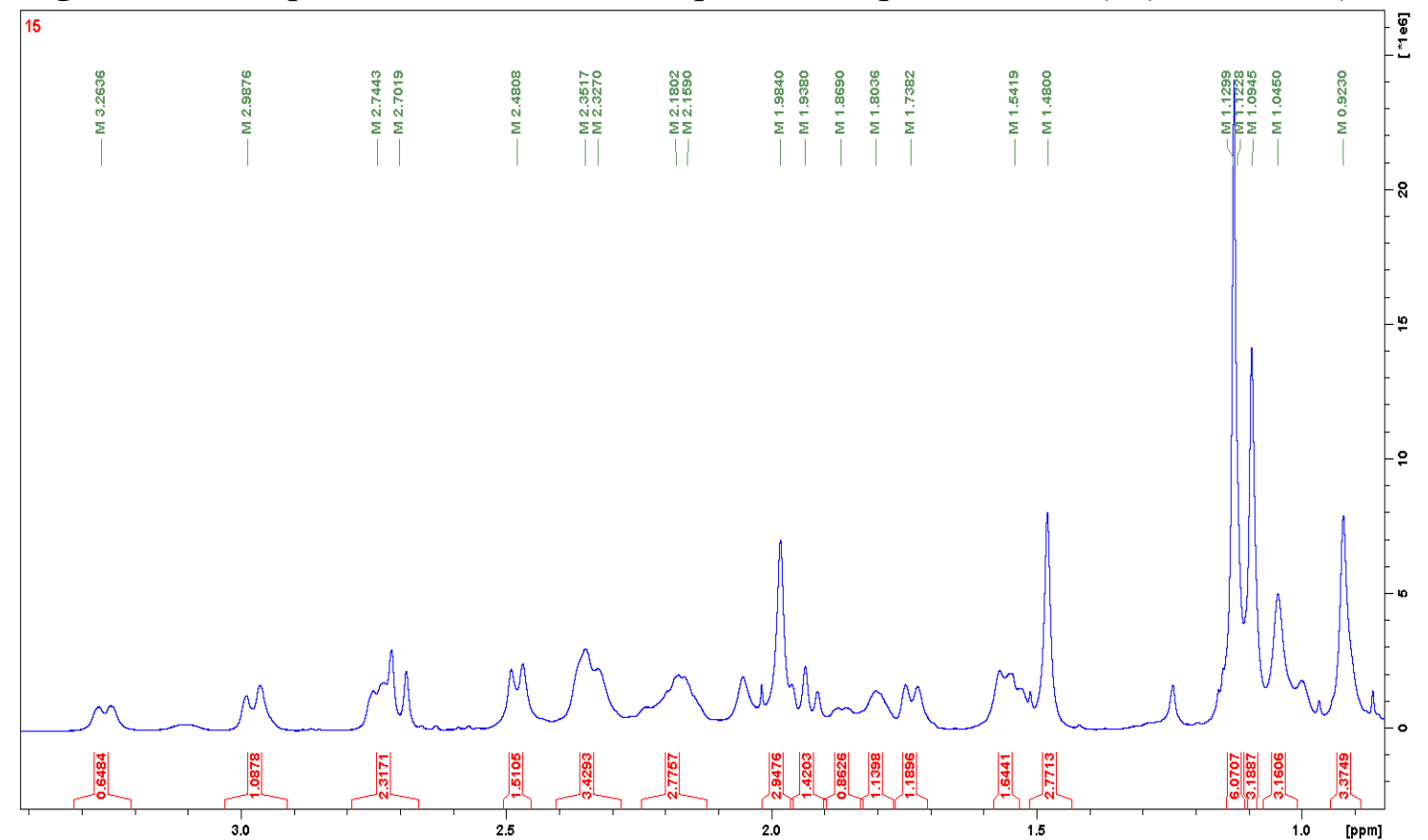

Figure S128. ${ }^{13} \mathrm{C}$ NMR spectrum of picraviane $\mathrm{O}(\mathbf{1 5})$ in $\mathrm{CDCl}_{3}(150 \mathrm{MHz})$.

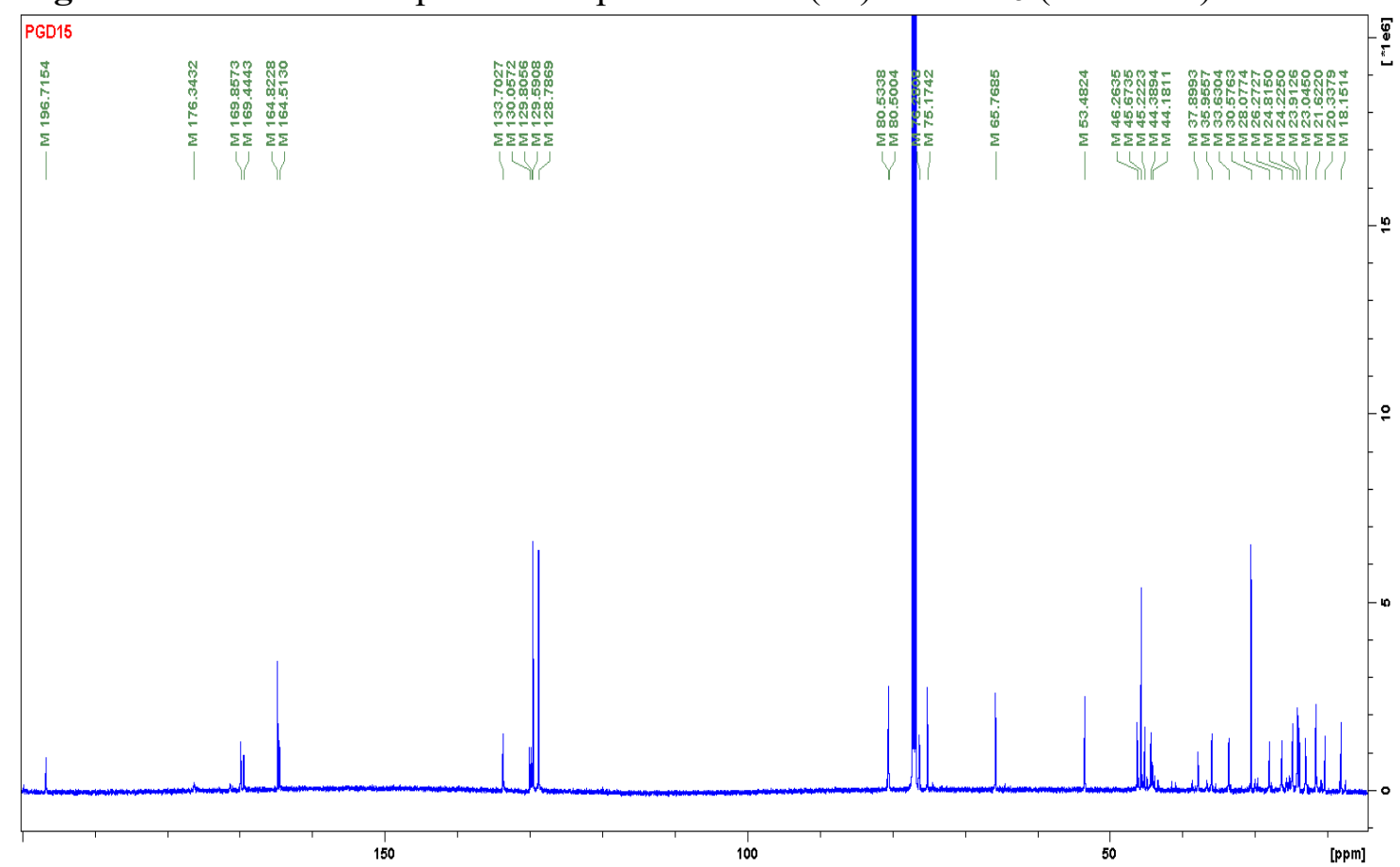


Figure S129. HSQC spectrum of picraviane $\mathrm{O}(\mathbf{1 5})$ in $\mathrm{CDCl}_{3}(600 \mathrm{MHz})$.

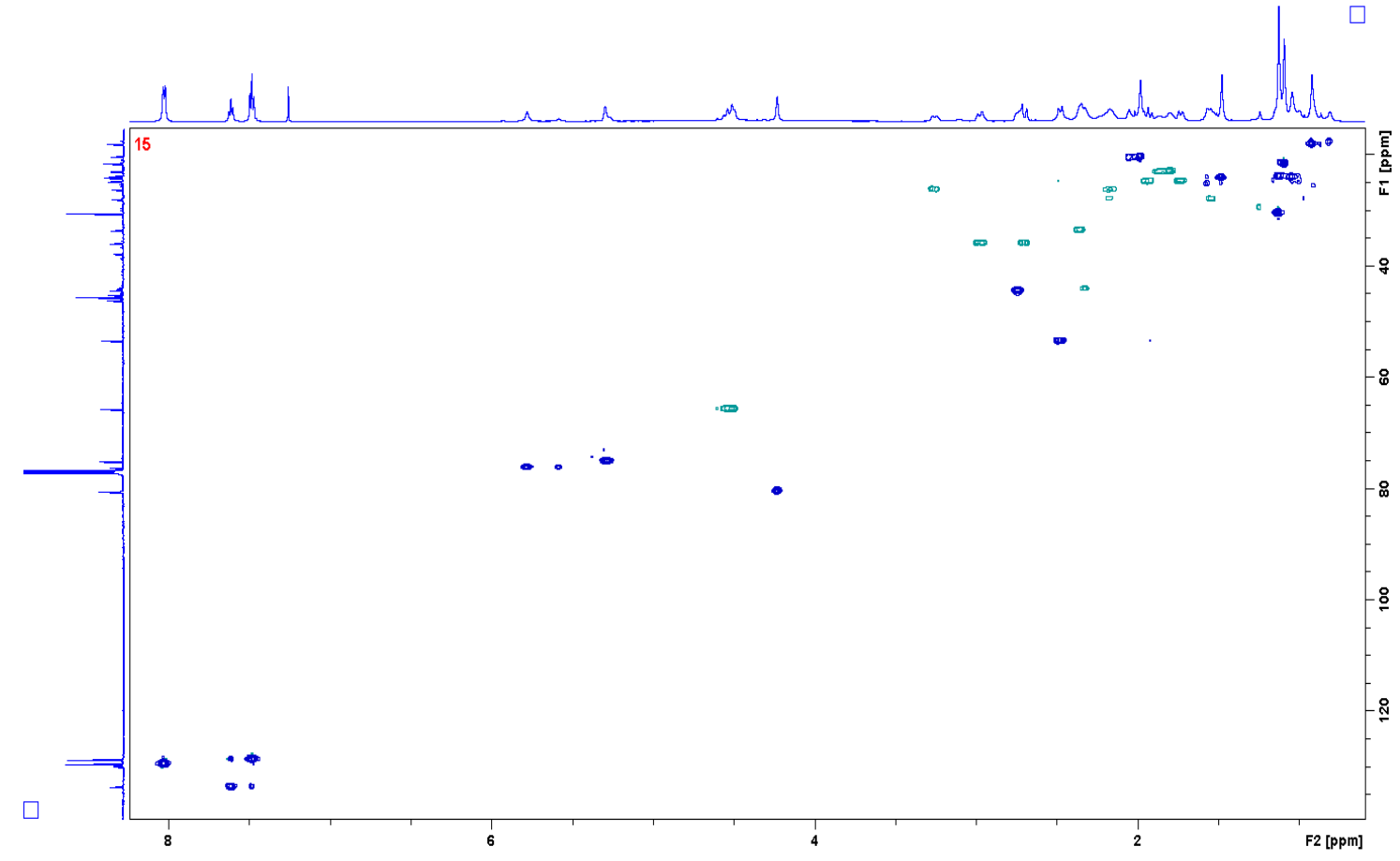

Figure S130. HMBC spectrum of picraviane $\mathrm{O}(\mathbf{1 5})$ in $\mathrm{CDCl}_{3}(600 \mathrm{MHz})$.

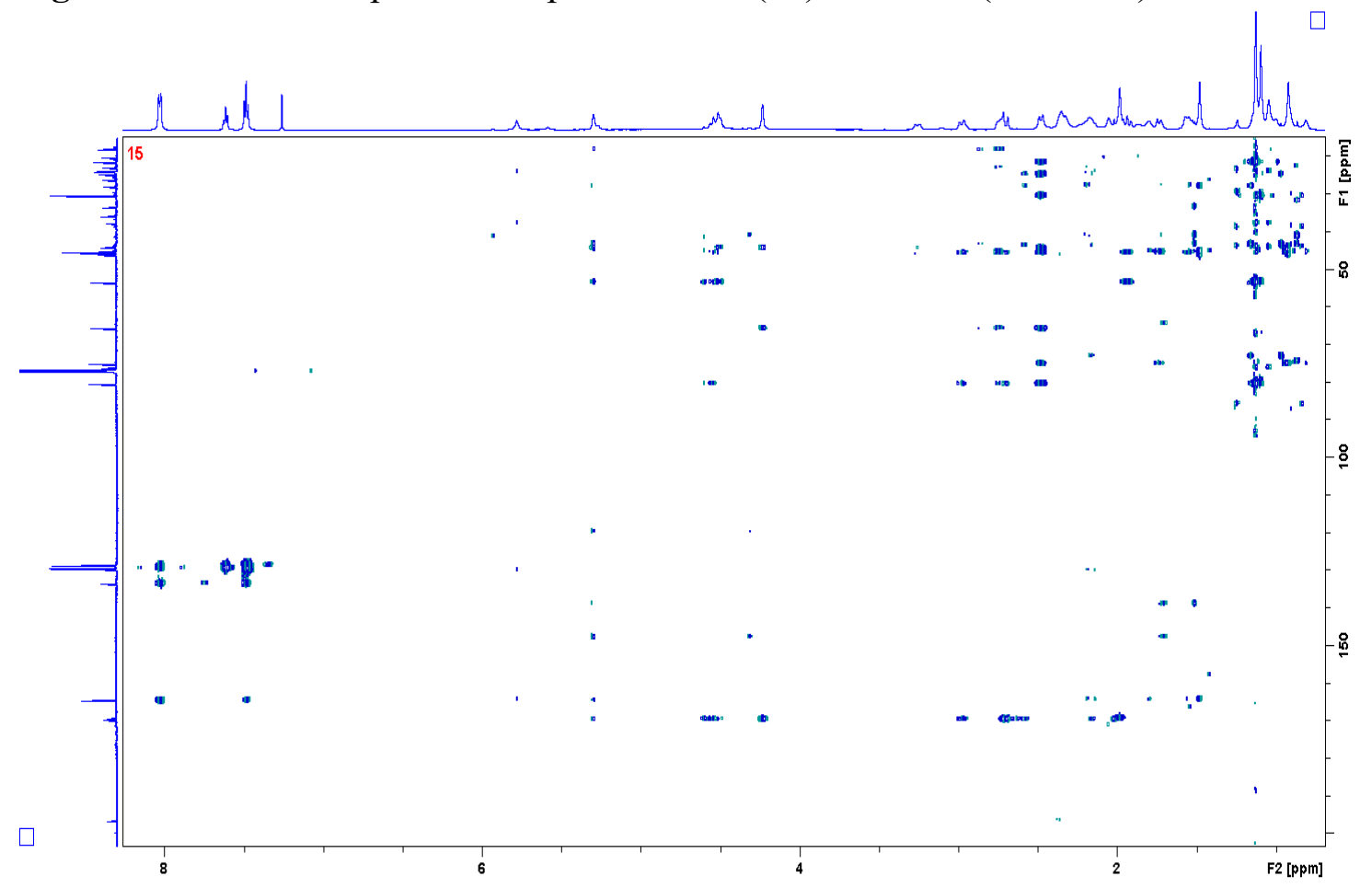


Figure S131. COSY spectrum of picraviane $\mathrm{O}(15)$ in $\mathrm{CDCl}_{3}(600 \mathrm{MHz})$.

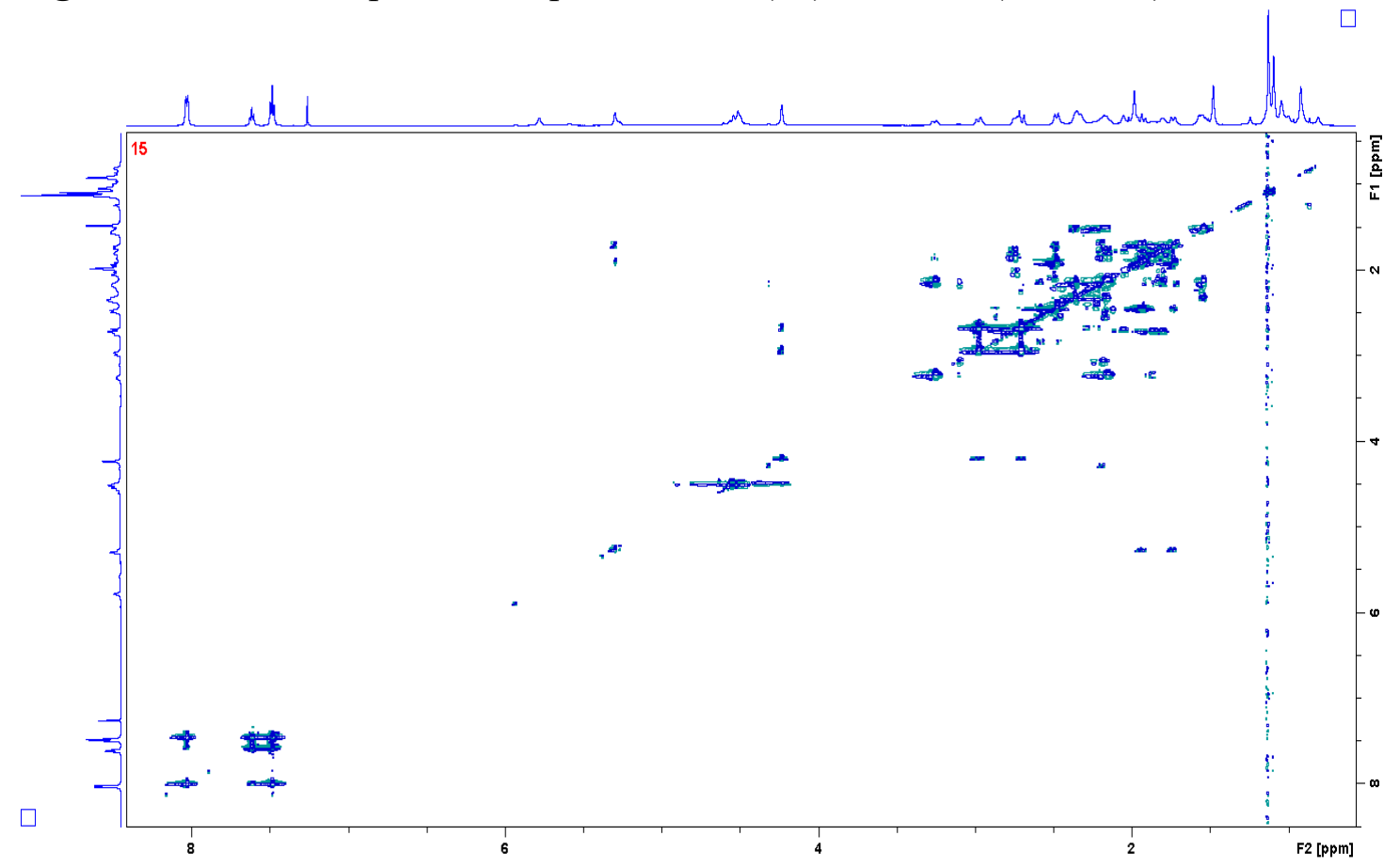

Figure S132. ROESY spectrum of picraviane $\mathrm{O}(\mathbf{1 5})$ in $\mathrm{CDCl}_{3}(600 \mathrm{MHz})$.

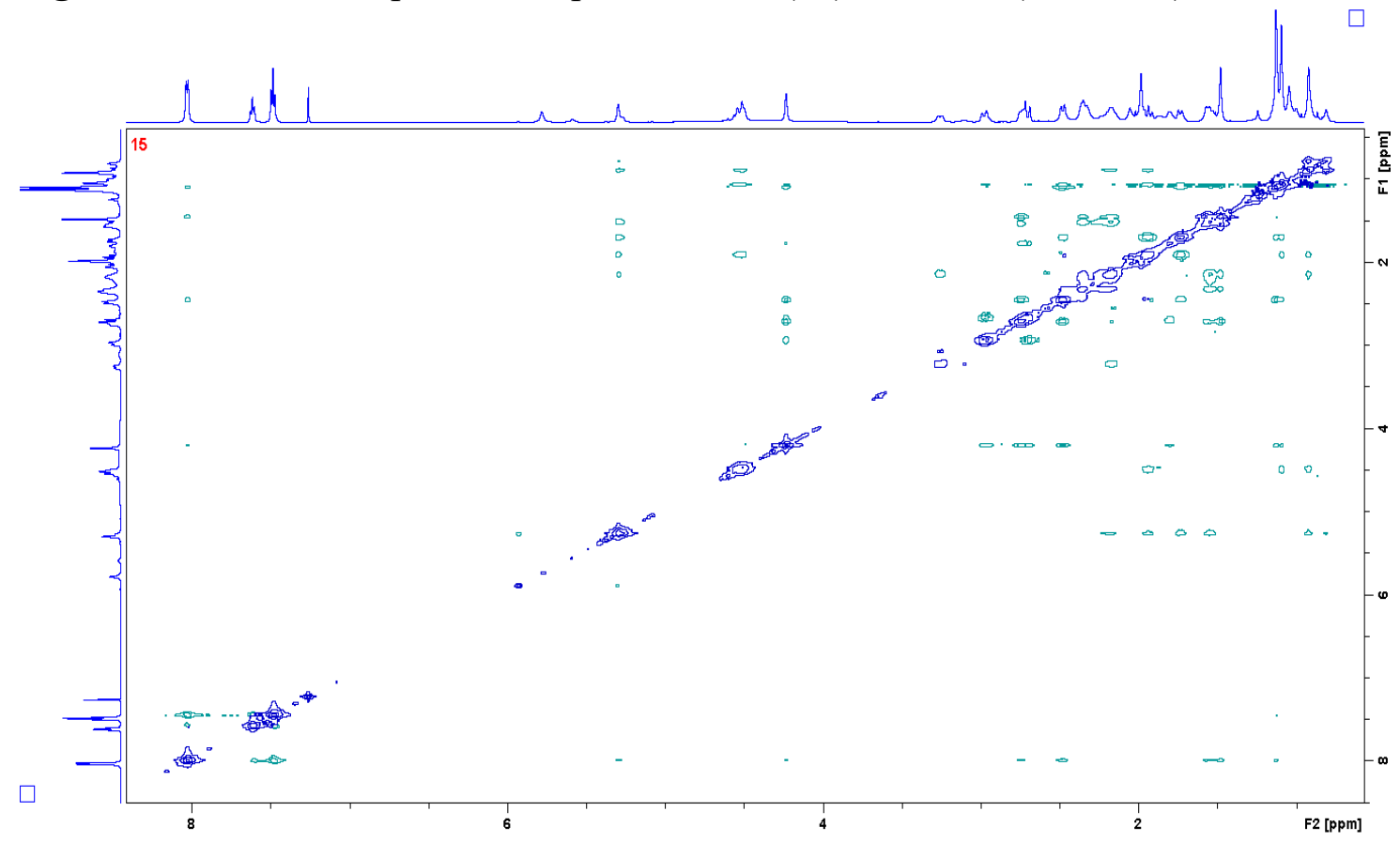


Figure S133. ${ }^{1} \mathrm{H}$ NMR spectrum of picraviane $\mathrm{T}(\mathbf{2 1})$ in $\mathrm{CDCl}_{3}(600 \mathrm{MHz})$.

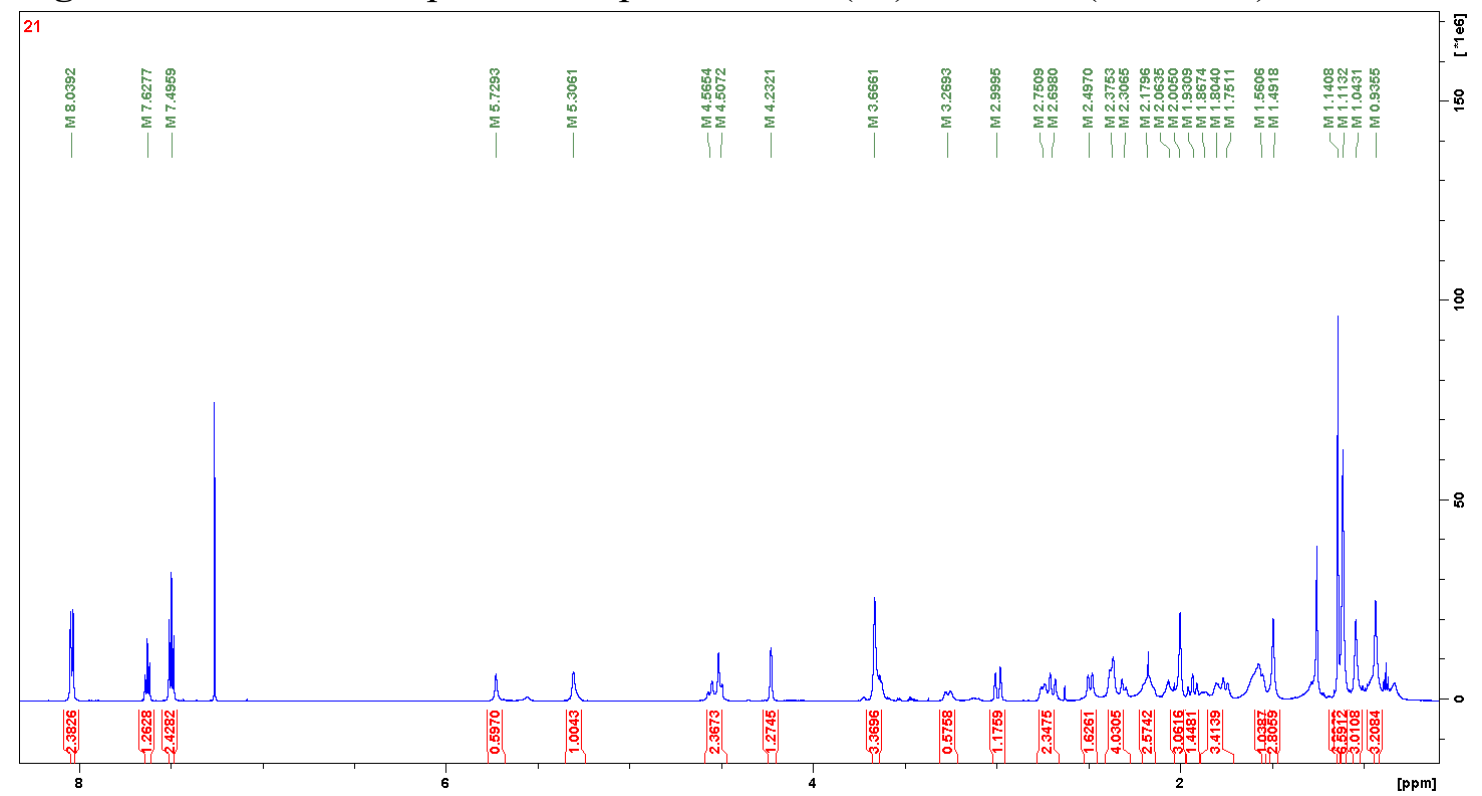

Figure S134. Expansion of the ${ }^{1} \mathrm{H}$ NMR spectrum of picraviane $\mathrm{T}(\mathbf{2 1})$ in $\mathrm{CDCl}_{3}(600 \mathrm{MHz})$.

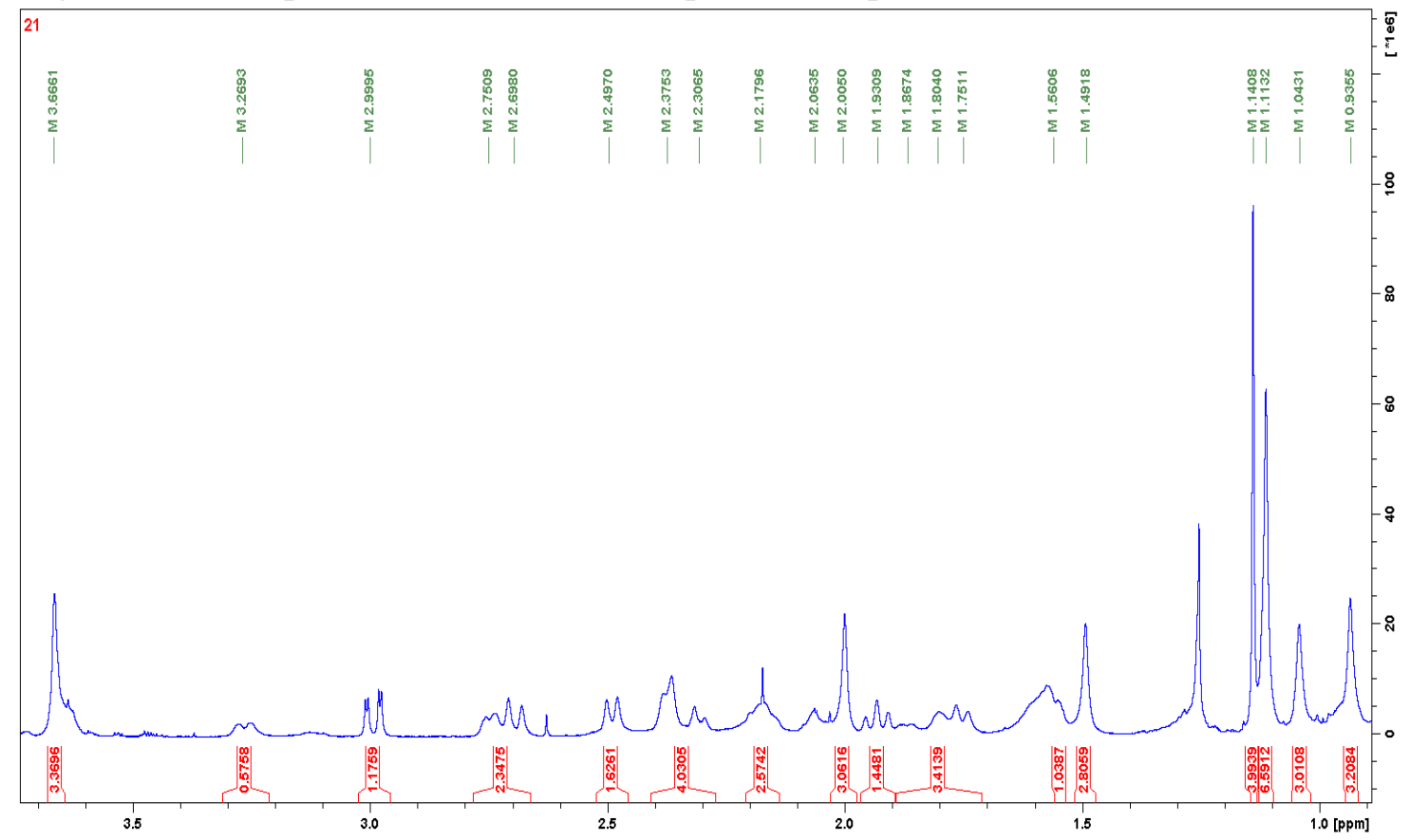


Figure S135. ${ }^{13} \mathrm{C}$ NMR spectrum of picraviane $\mathrm{T}(\mathbf{2 1})$ in $\mathrm{CDCl}_{3}(150 \mathrm{MHz})$.

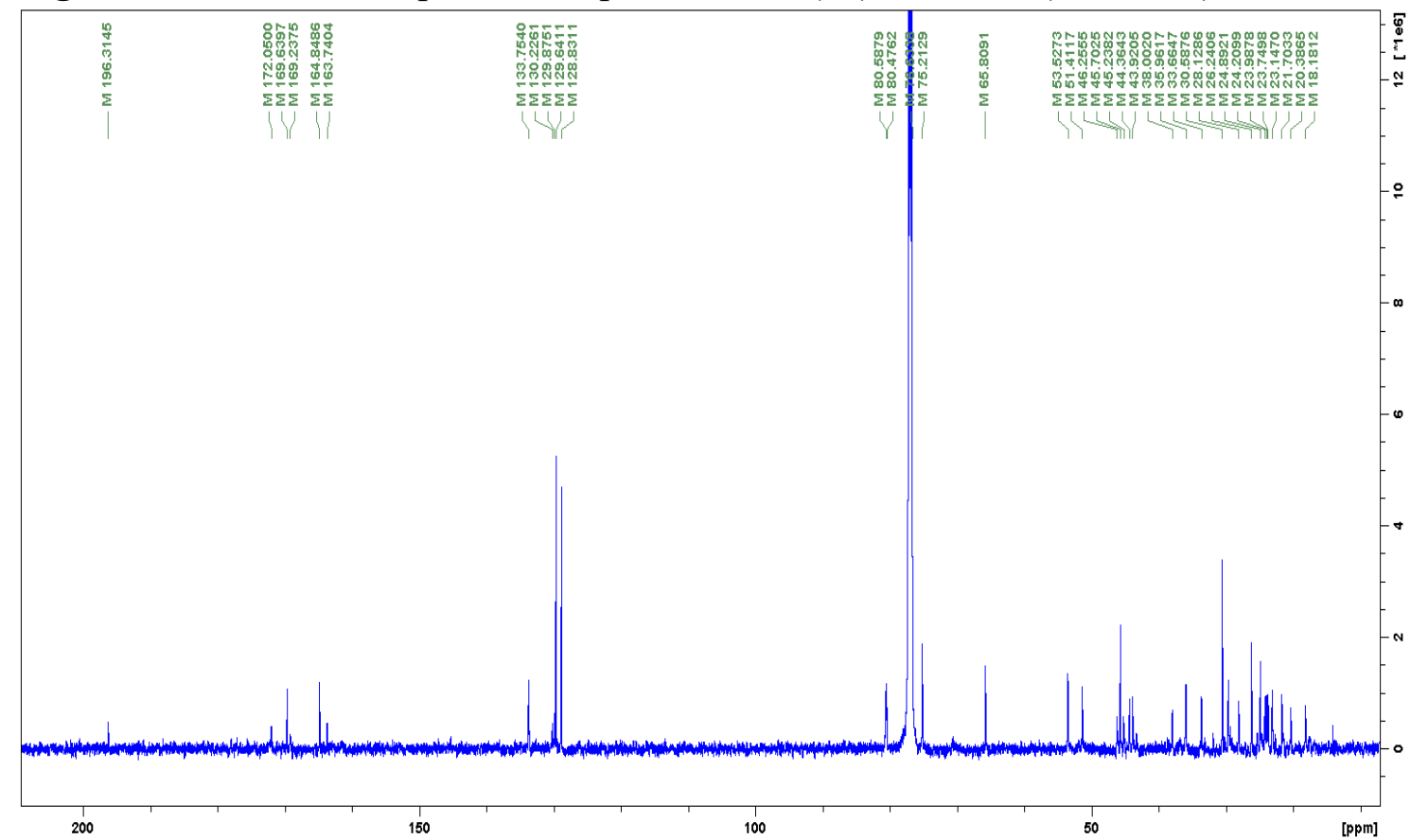

Figure S136. HSQC spectrum of picraviane $\mathrm{T}(\mathbf{2 1})$ in $\mathrm{CDCl}_{3}(600 \mathrm{MHz})$.

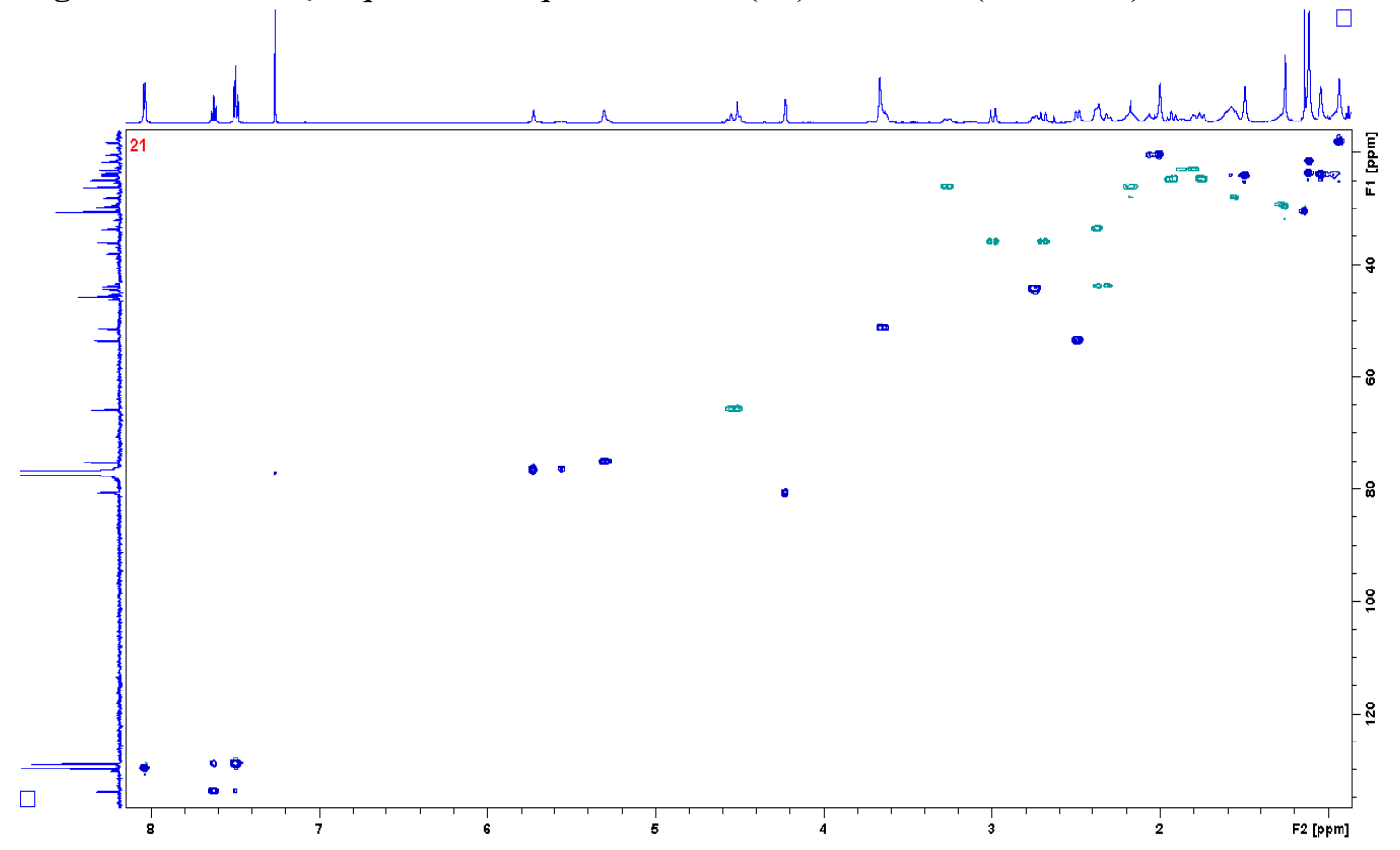


Figure S137. HMBC spectrum of picraviane T (21) in $\mathrm{CDCl}_{3}(600 \mathrm{MHz})$.

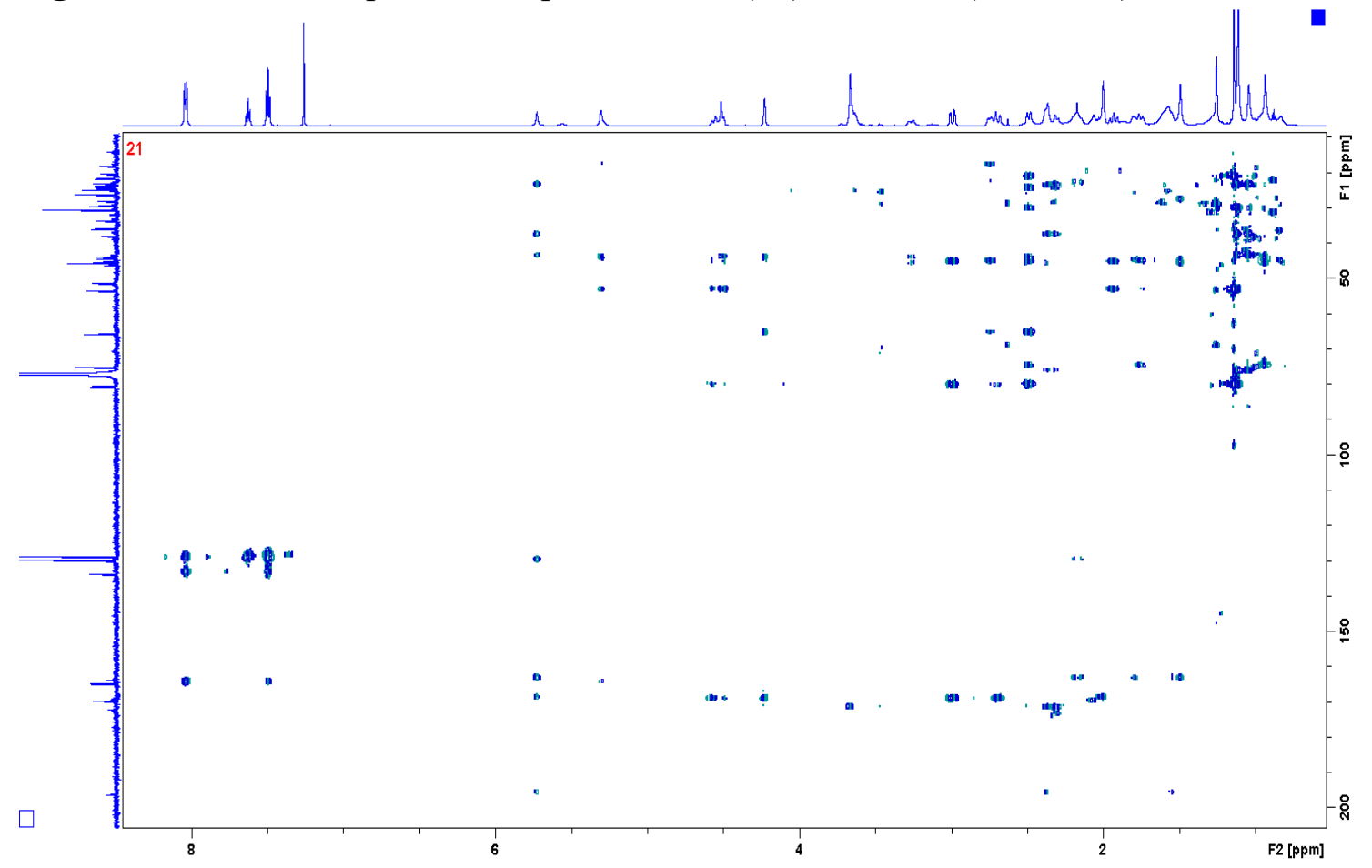

Figure S138. COSY spectrum of picraviane T (21) in $\mathrm{CDCl}_{3}(600 \mathrm{MHz})$.

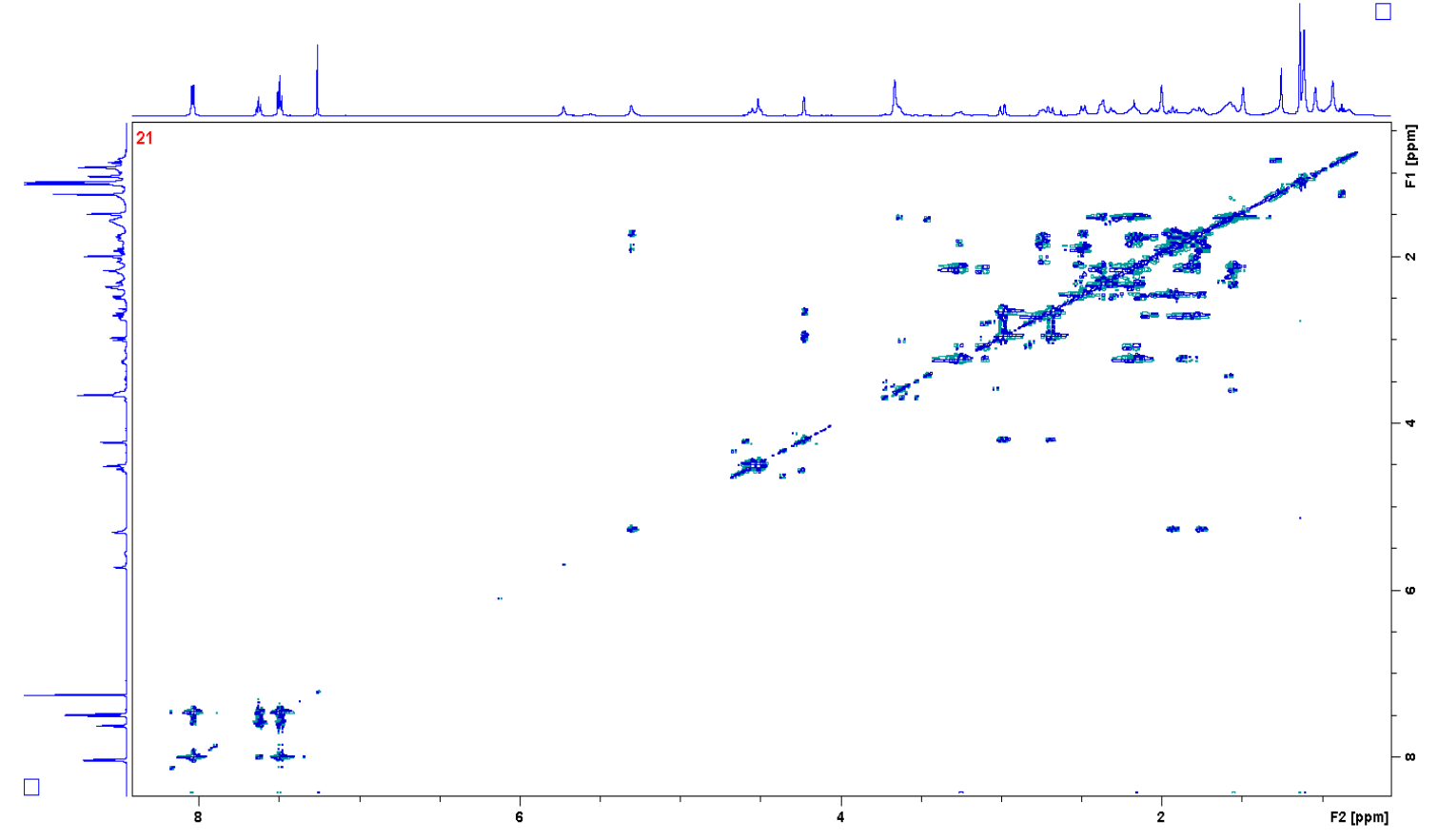


Figure S139. NOESY spectrum of picraviane $\mathrm{T}(\mathbf{2 1})$ in $\mathrm{CDCl}_{3}(600 \mathrm{MHz})$.

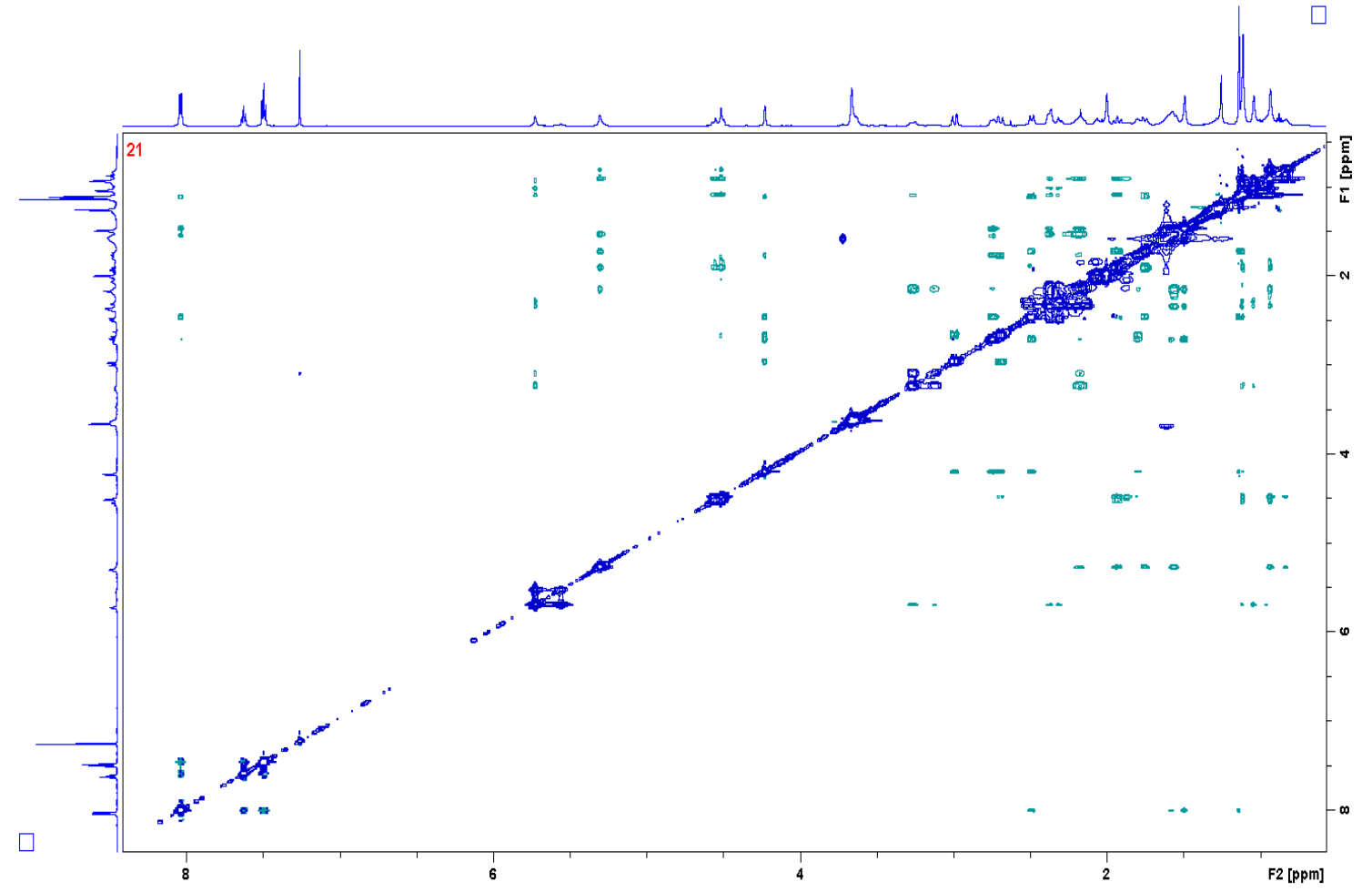

Figure S140. Structures of the three lowest-energy conformers of $(1 S, 5 R, 7 R, 8 R, 9 S, 10 R, 14 R, 19 S)-15$ at the B3LYP/PCM(MeOH)/TZVP level.

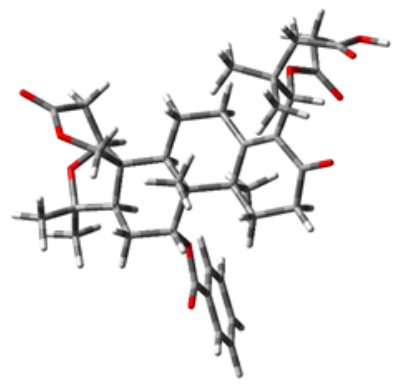

Conf. $1,0.0 \mathrm{kcal}^{-1}(71,5 \%)$

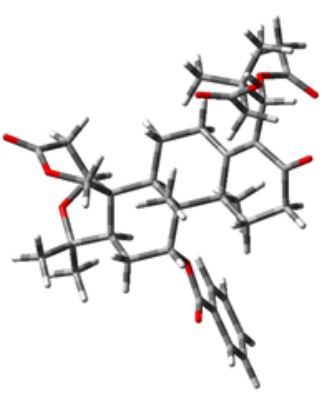

Conf. $2,+0.8 \mathrm{kcal}^{-1}(18,5 \%)$

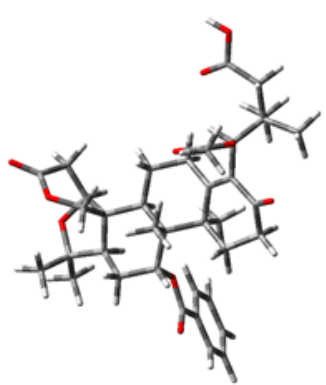

Conf. $3,+1.1 \mathrm{kcal}^{-1}(10 \%)$ 
Figure S141. Comparison of the experimental and calculated UV and ECD spectra of $(1 S, 5 R, 7 R, 8 R, 9 S, 10 R, 14 R, 19 S)-15$ (blue trace) and its enantiomer (red trace). Experimental spectra were acquired in methanol, and UV and ECD spectra were calculated at the [CAM$\mathrm{B} 3 \mathrm{LYP} / \mathrm{PCM}(\mathrm{MeOH}) / \mathrm{TZVP}]$ level for the Boltzmann average of the three lowest-energy conformers identified for $(1 S, 5 R, 7 R, 8 R, 9 S, 10 R, 14 R, 19 S)-\mathbf{1 5}$.

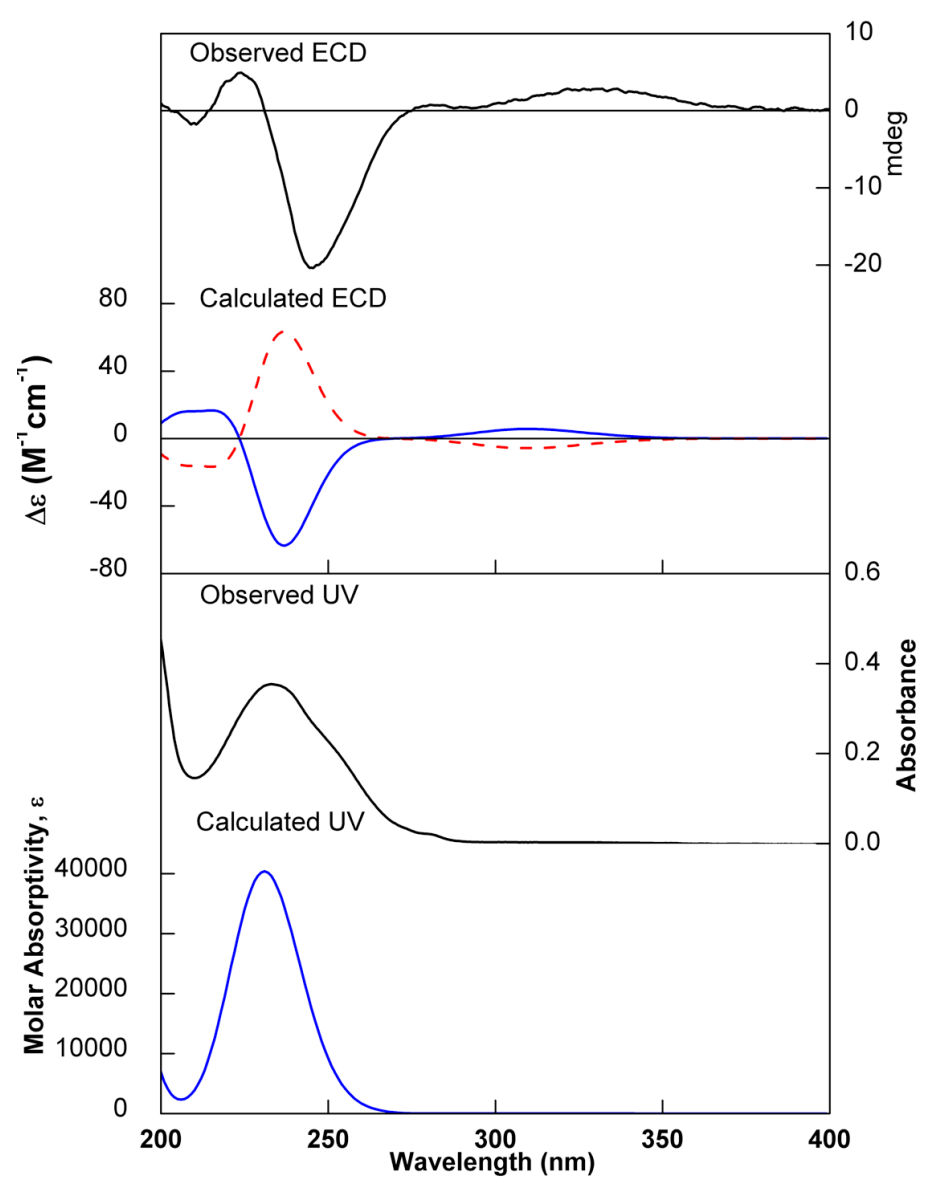


Figure S142. Structures of lowest-energy conformations and ECD spectra of $(1 S, 5 R, 7 R, 8 R, 9 S, 10 R, 14 R, 19 R)-15$. Left: Structures, relative energies and Boltzman populations of the lowest-energy conformers identified for $(1 S, 5 R, 7 R, 8 R, 9 S, 10 R, 14 R, 19 R)$ 15. Right: Experimental and calculated ECD spectra for $(1 S, 5 R, 7 R, 8 R, 9 S, 10 R, 14 R, 19 R)-15$ at the CAM-B3LYP/PCM(MeOH)/TZVP level.
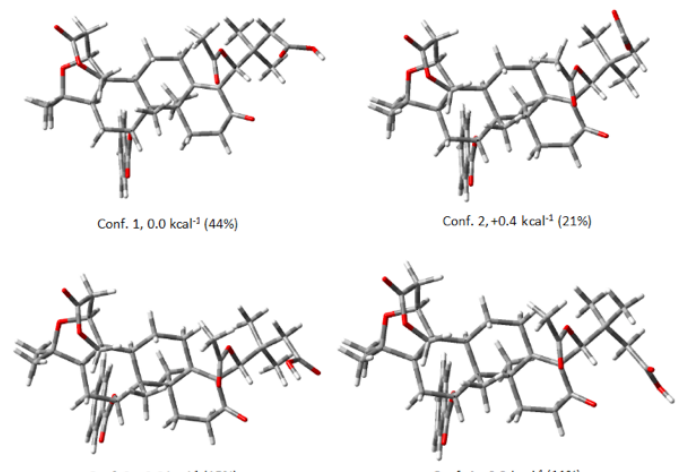

Conf. $3,+0.6 \mathrm{kcal}^{-1}(15 \%)$

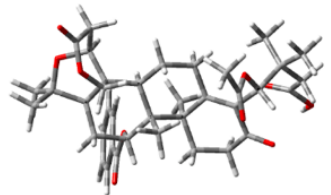

Conf. $5,+0.9 \mathrm{kcal}^{-1}(9 \%)$

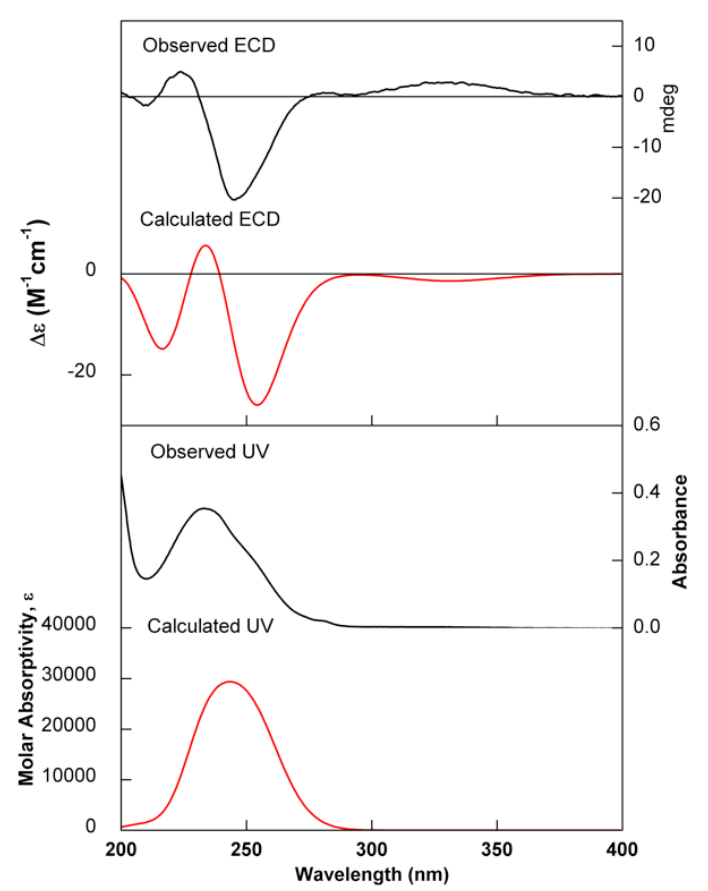


Figure S143. Comparison of the experimental ECD spectra of 15 and 21, which differ only by a methoxy group at C-22.

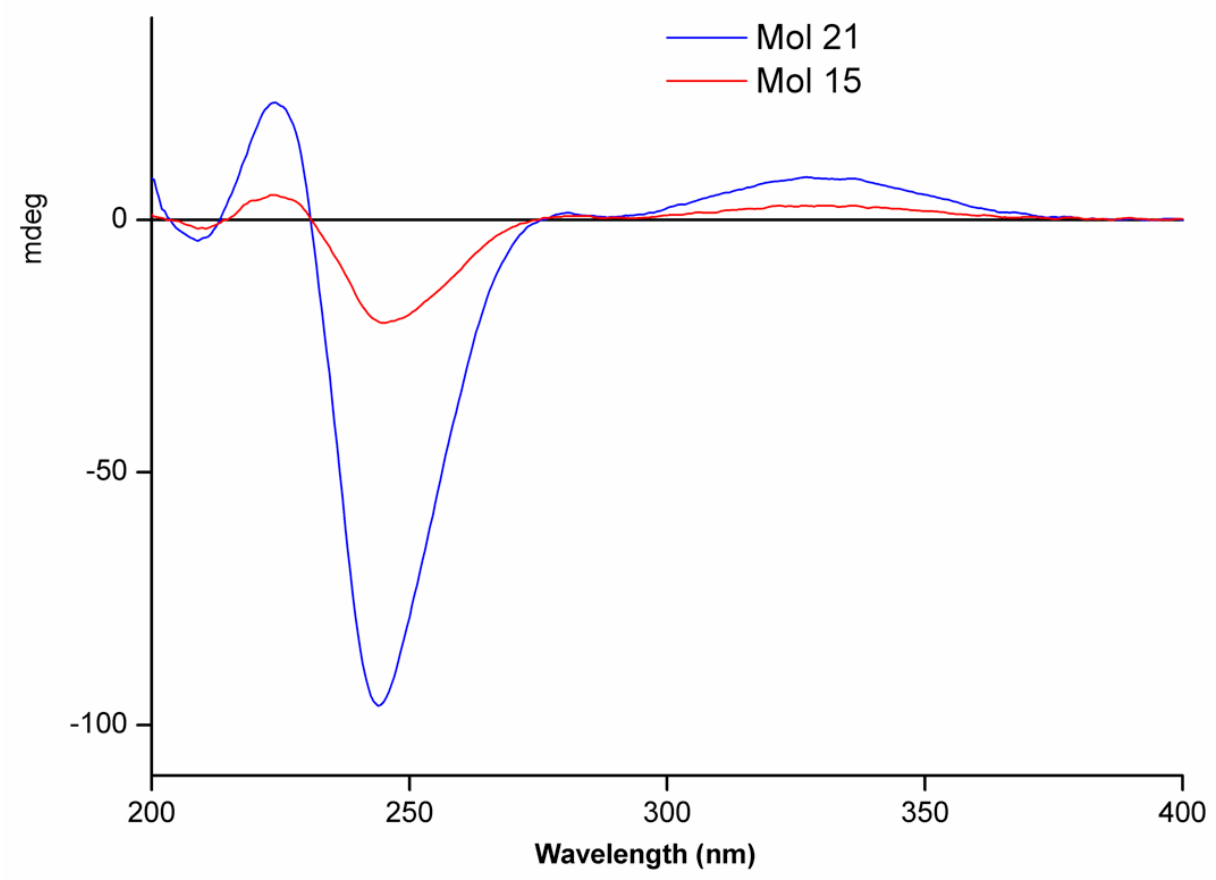

Figure S144. ${ }^{1} \mathrm{H}$ NMR spectrum of picraviane C (1) in $\mathrm{CDCl}_{3}(600 \mathrm{MHz})$.

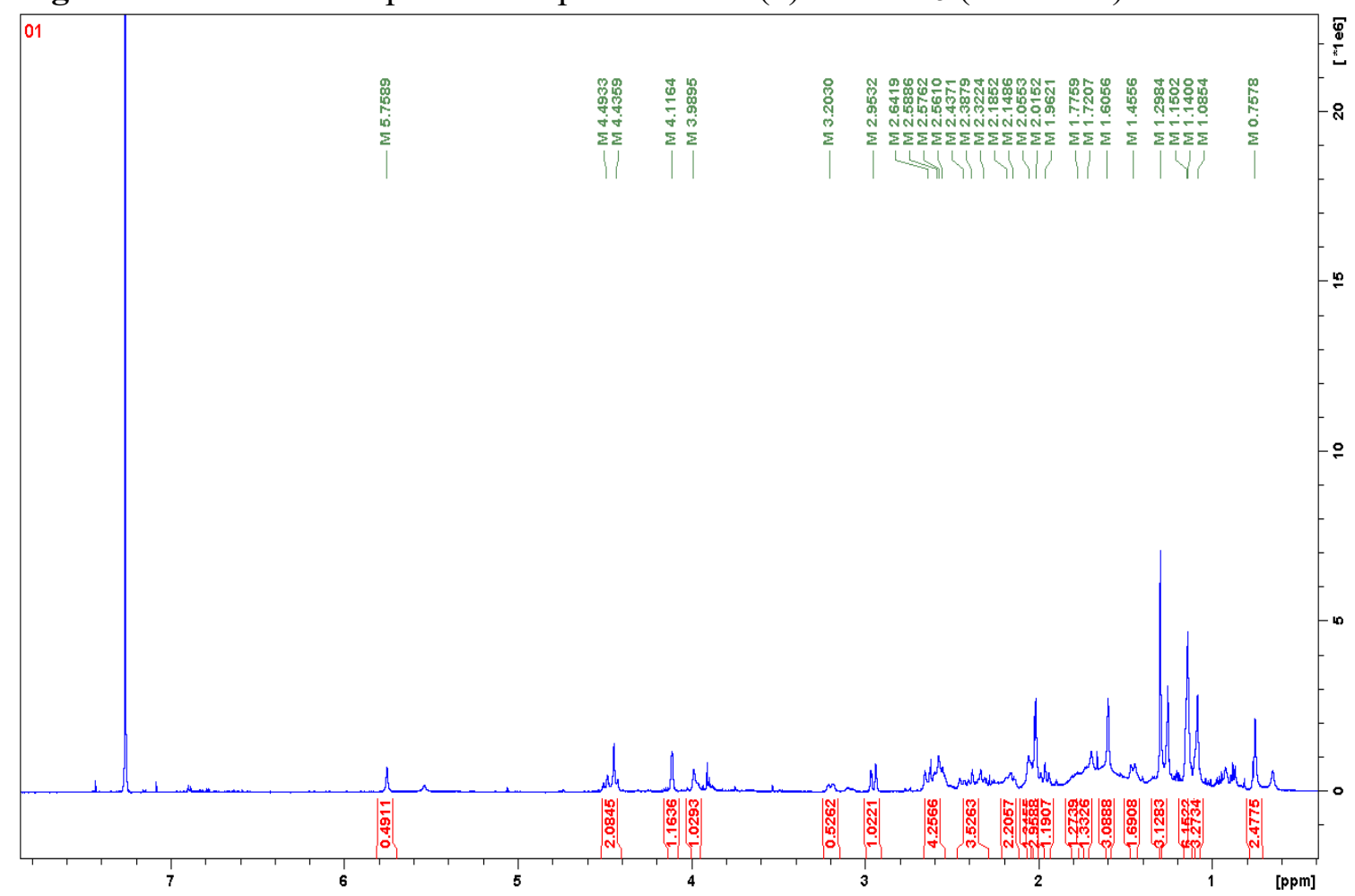


Figure S145. Expansion of the ${ }^{1} \mathrm{H}$ NMR spectrum of picraviane $\mathrm{C}(1)$ in $\mathrm{CDCl}_{3}(600 \mathrm{MHz})$.

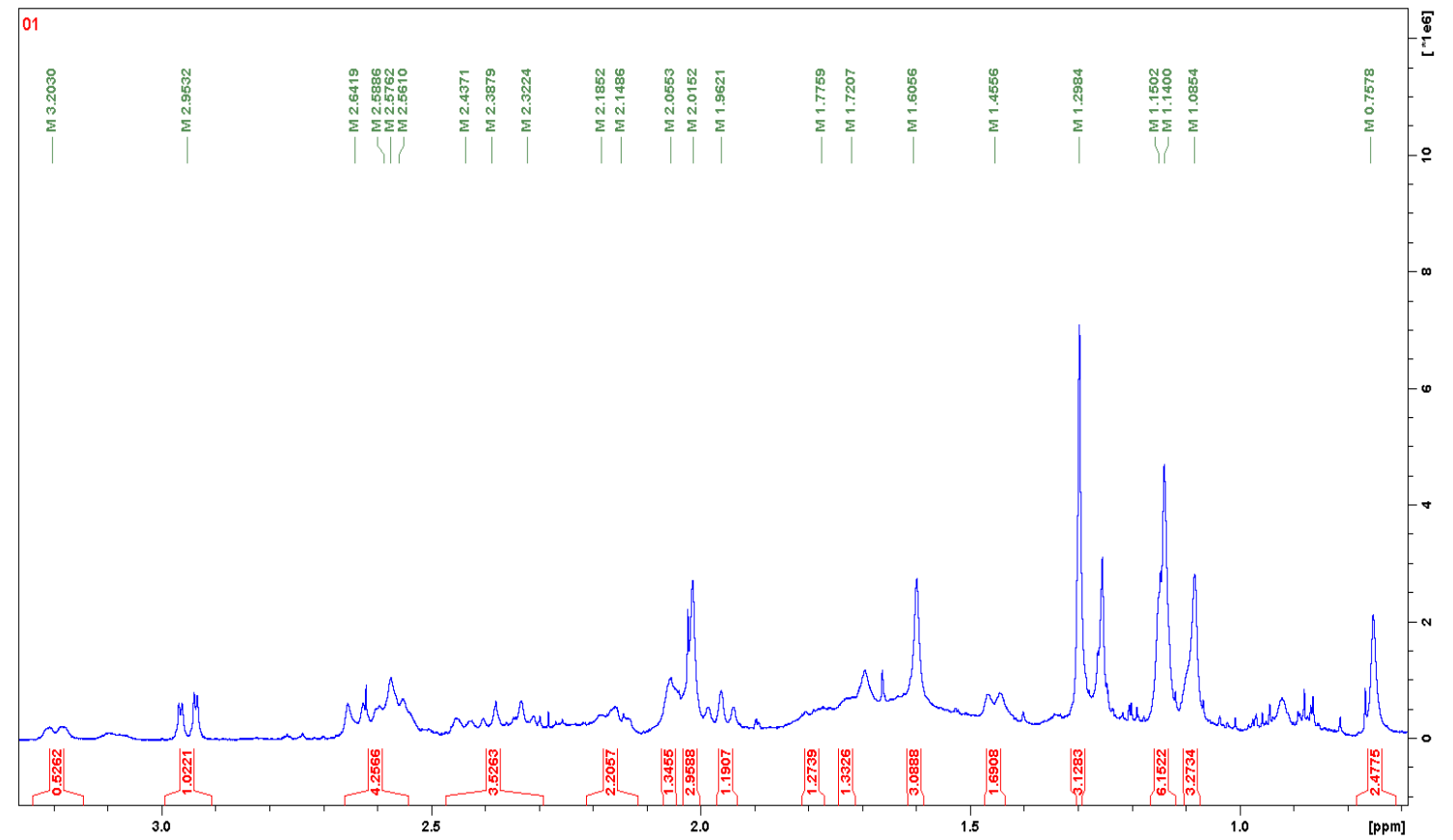

Figure S146. HSQC spectrum of picraviane C (1) in $\mathrm{CDCl}_{3}(600 \mathrm{MHz})$.

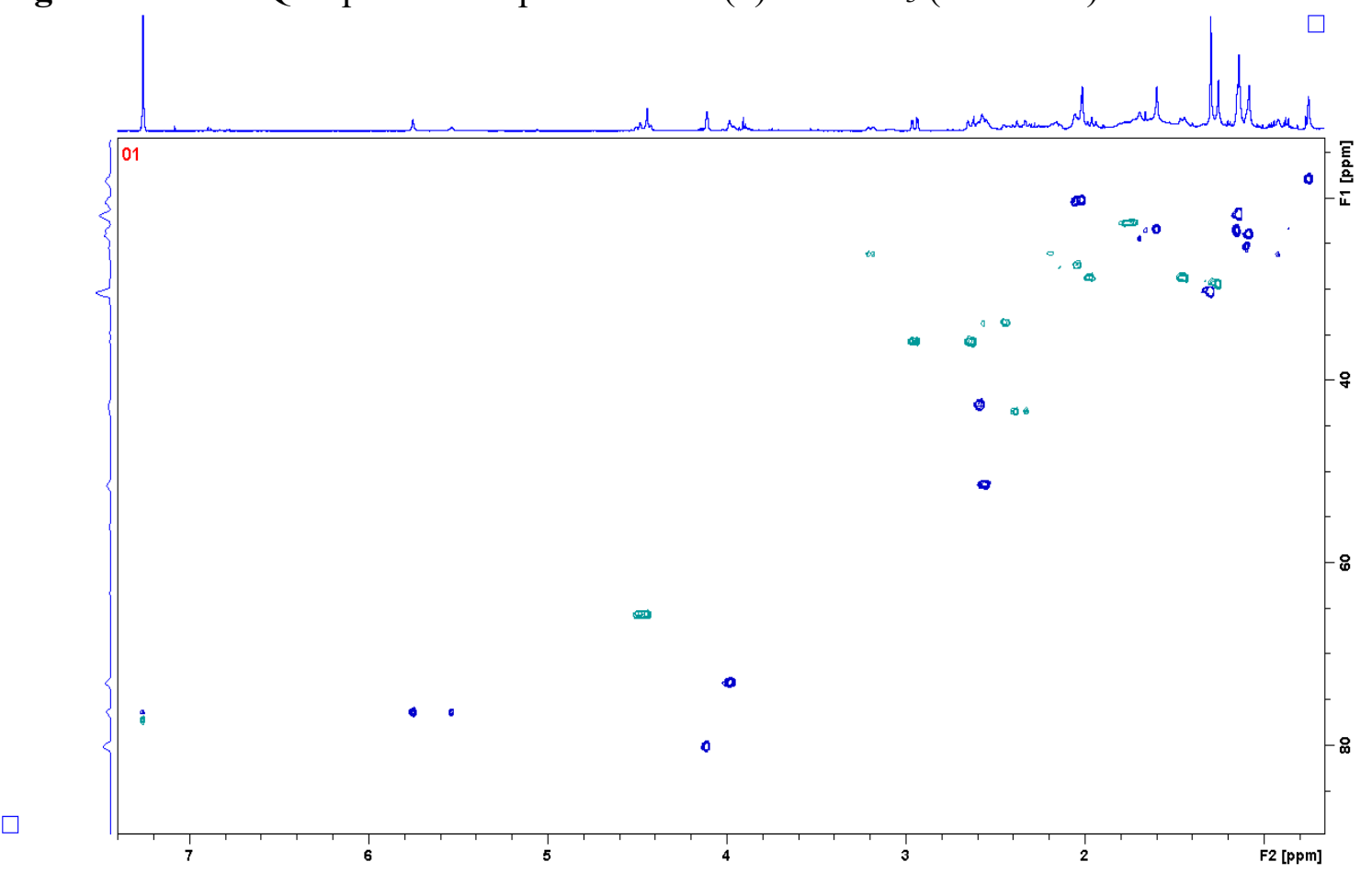


Figure S147. HMBC spectrum of picraviane C (1) in $\mathrm{CDCl}_{3}(600 \mathrm{MHz})$.

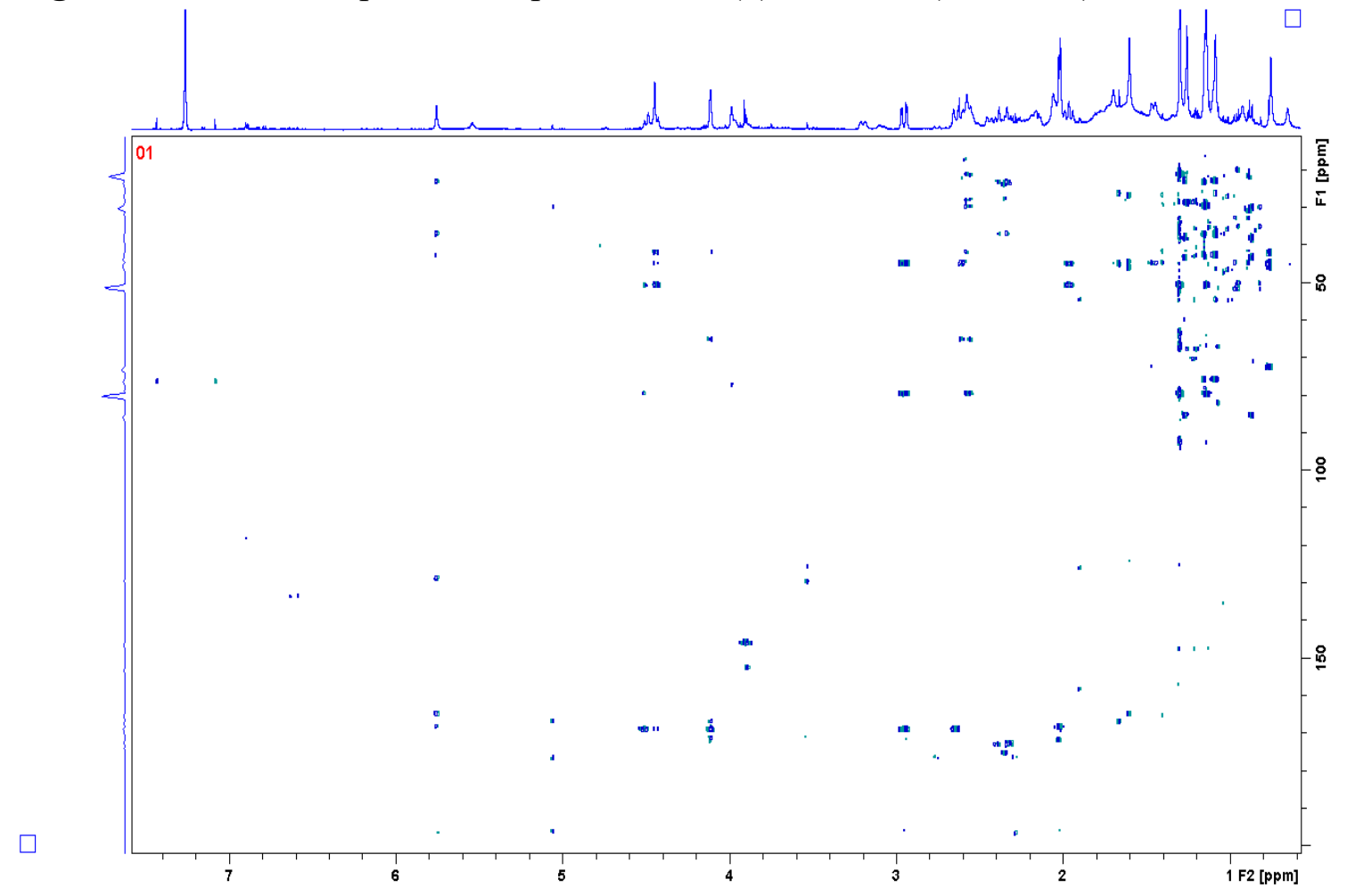

Figure S148. COSY spectrum of picraviane C (1) in $\mathrm{CDCl}_{3}(600 \mathrm{MHz})$.

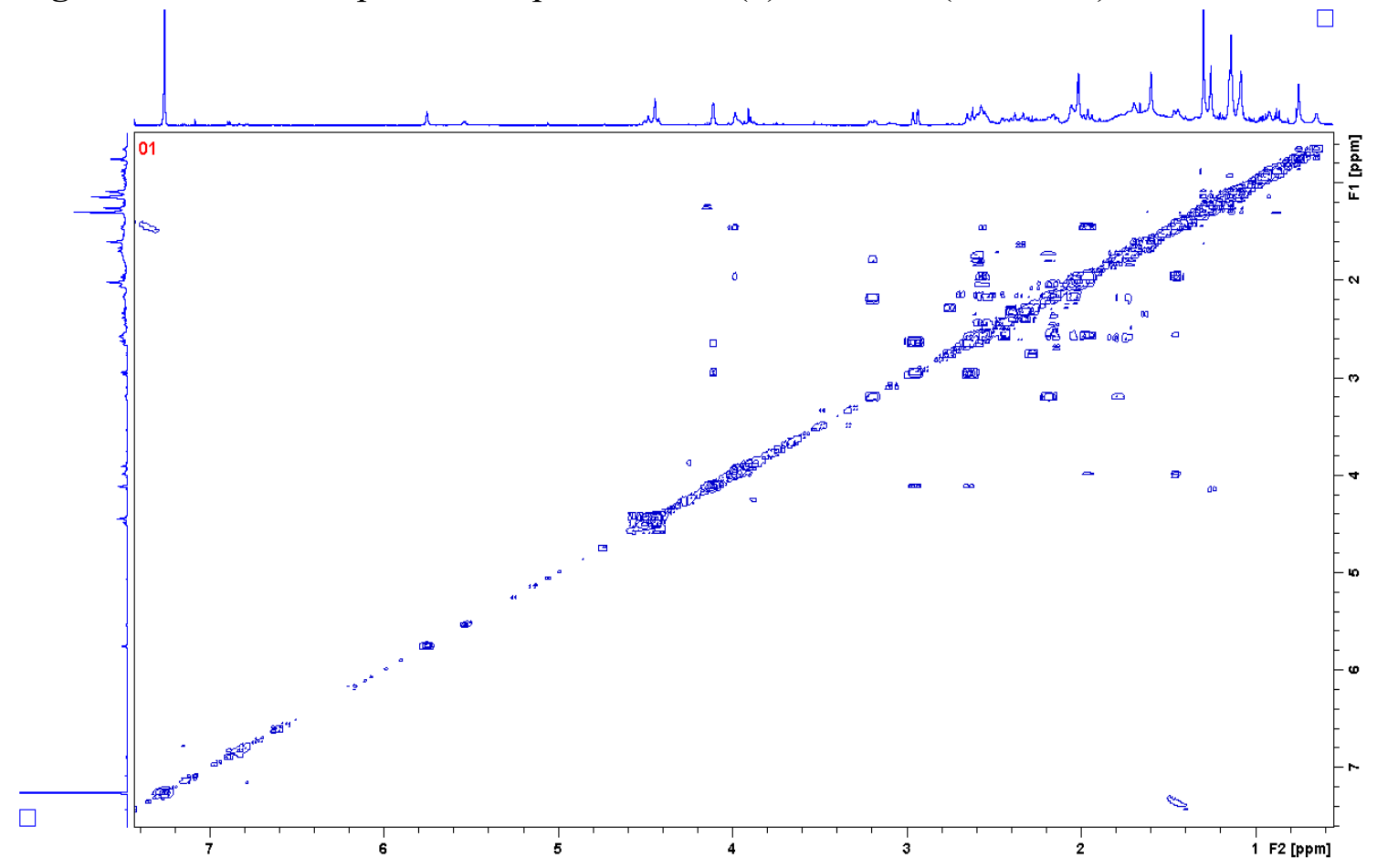


Figure S149. ROESY spectrum of picraviane C (1) in $\mathrm{CDCl}_{3}(600 \mathrm{MHz})$.

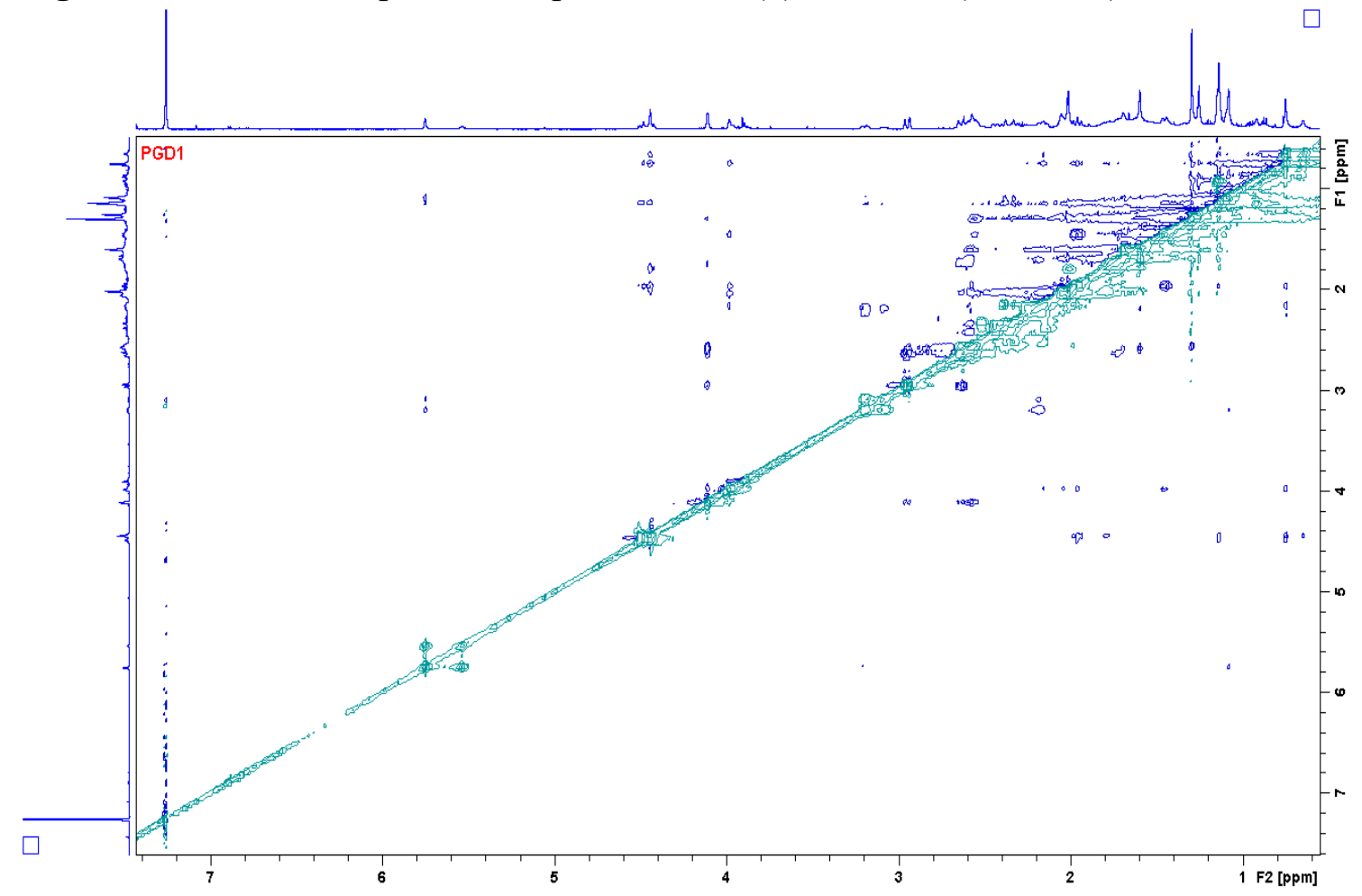

Figure S150. ${ }^{1} \mathrm{H}$ NMR spectrum of picraviane $\mathrm{I}(8)$ in $\mathrm{CDCl}_{3}(600 \mathrm{MHz})$.

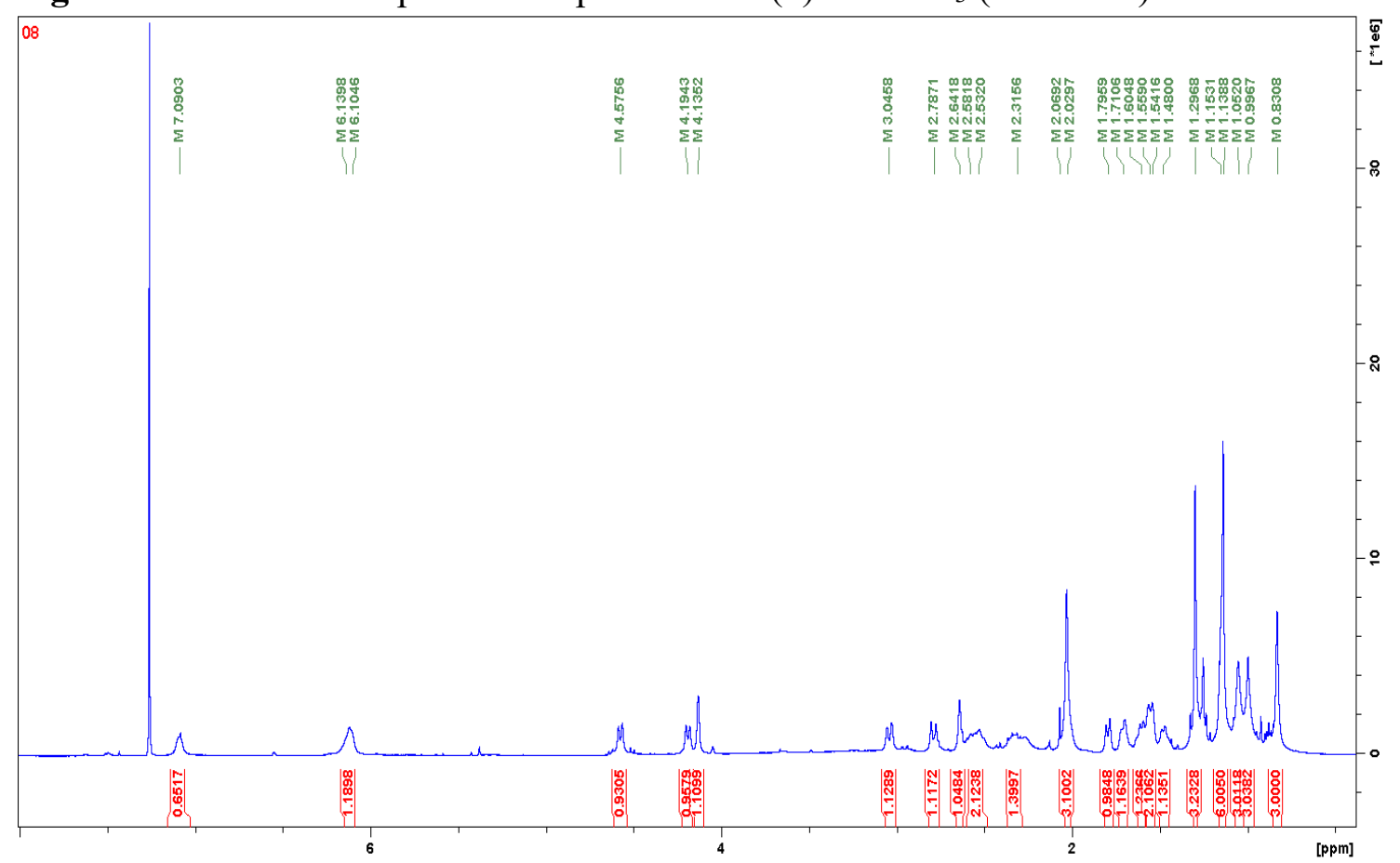


Figure S151. Expansion of the ${ }^{1} \mathrm{H}$ NMR spectrum of picraviane $\mathrm{I}(8)$ in $\mathrm{CDCl}_{3}(600 \mathrm{MHz})$.

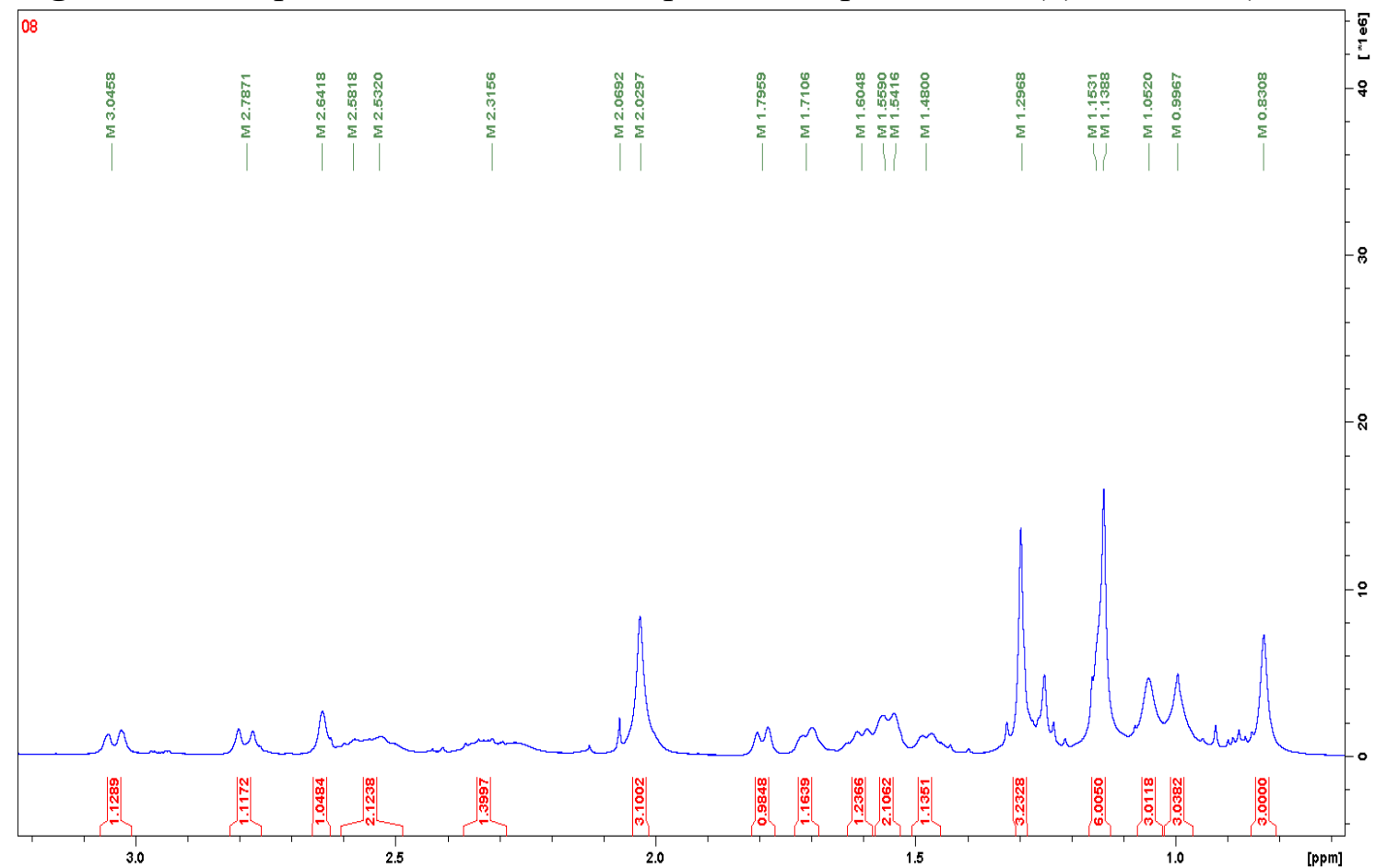

Figure S152. ${ }^{13} \mathrm{C}$ NMR spectrum of picraviane $\mathrm{I}(\mathbf{8})$ in $\mathrm{CDCl}_{3}(150 \mathrm{MHz})$.

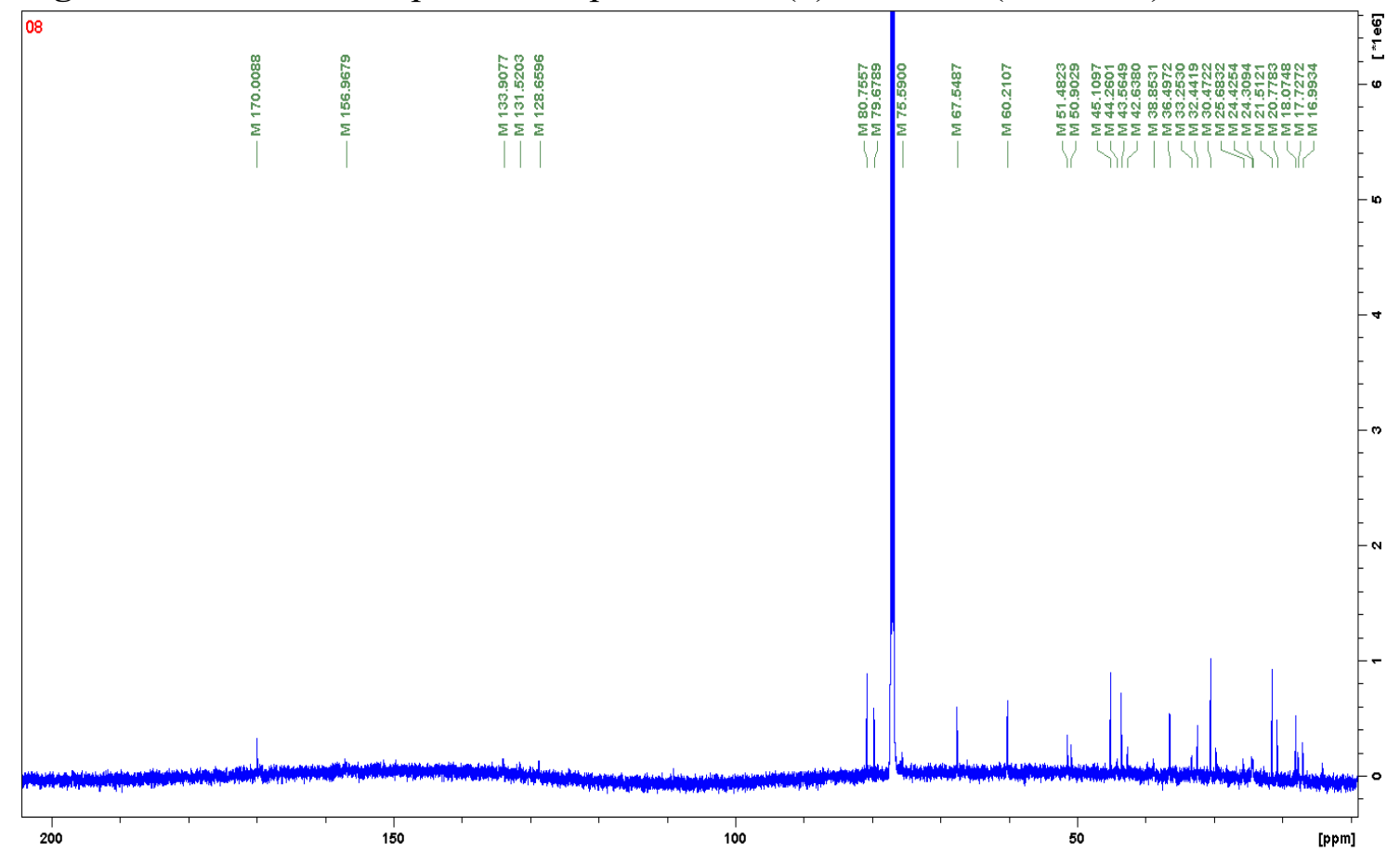


Figure S153. HSQC spectrum of picraviane I (8) in $\mathrm{CDCl}_{3}(600 \mathrm{MHz})$.

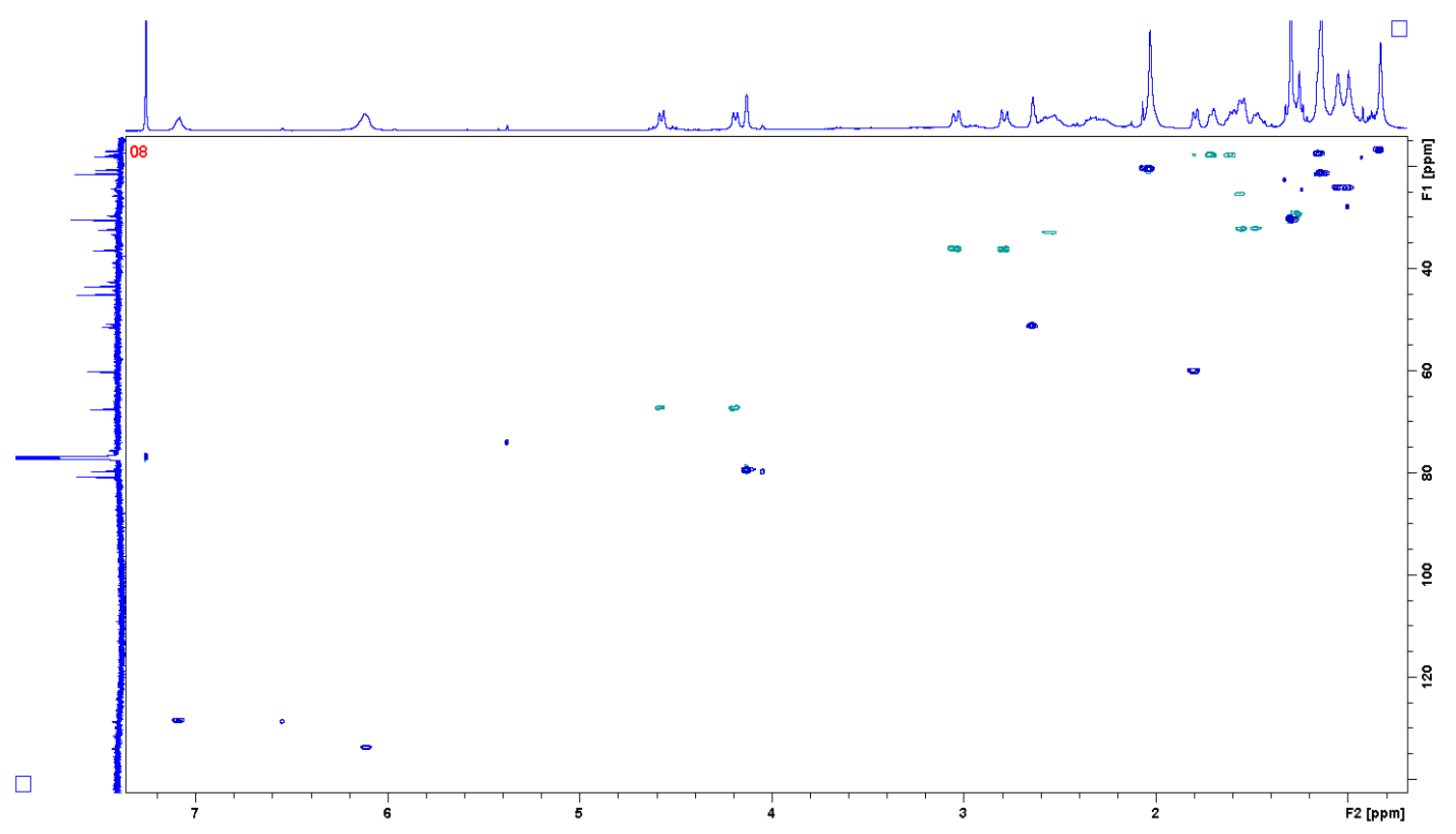

Figure S154. HMBC spectrum of picraviane I (8) in $\mathrm{CDCl}_{3}(600 \mathrm{MHz})$.

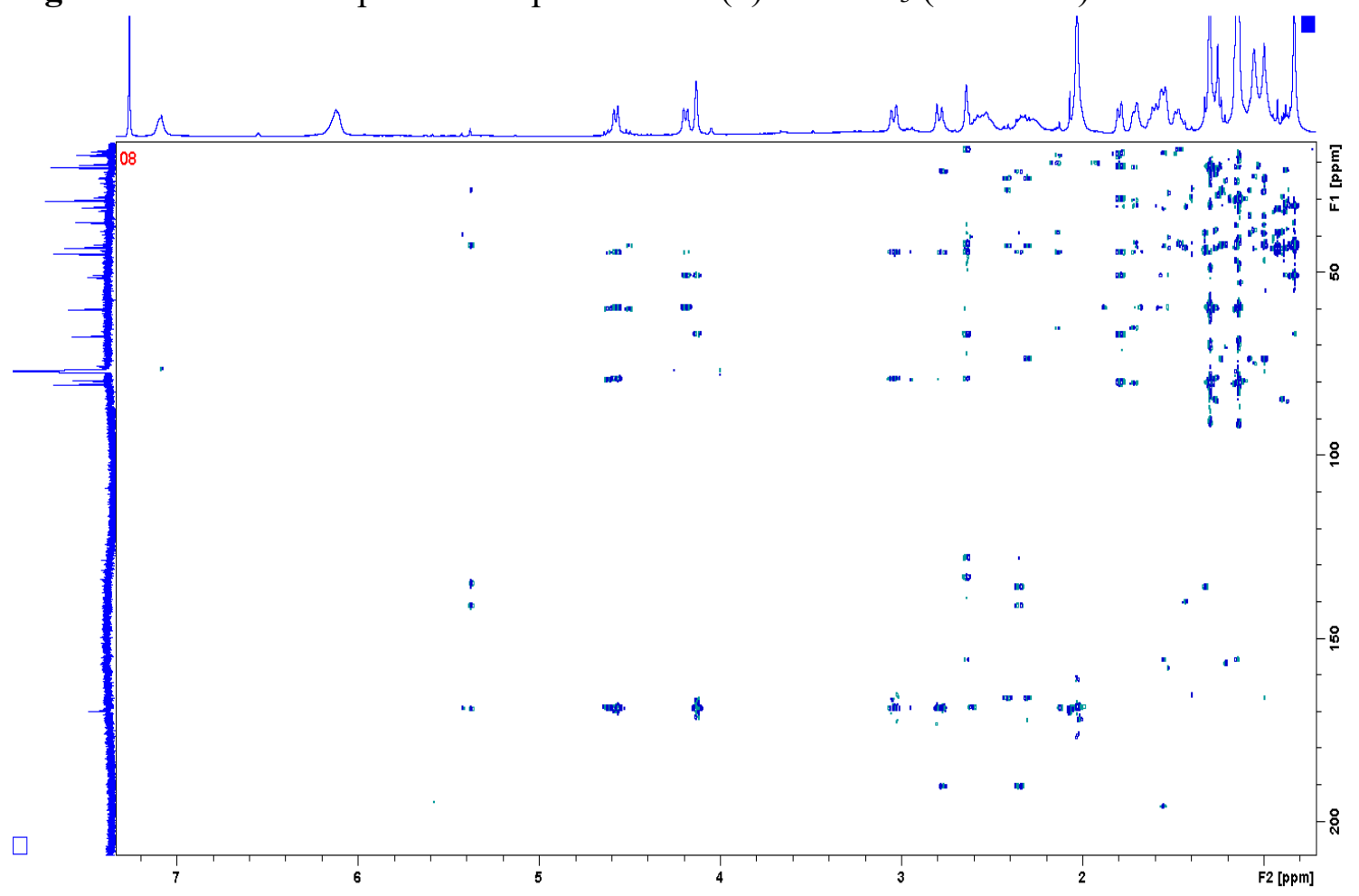

92 
Figure S155. COSY spectrum of picraviane I (8) in $\mathrm{CDCl}_{3}(600 \mathrm{MHz})$.

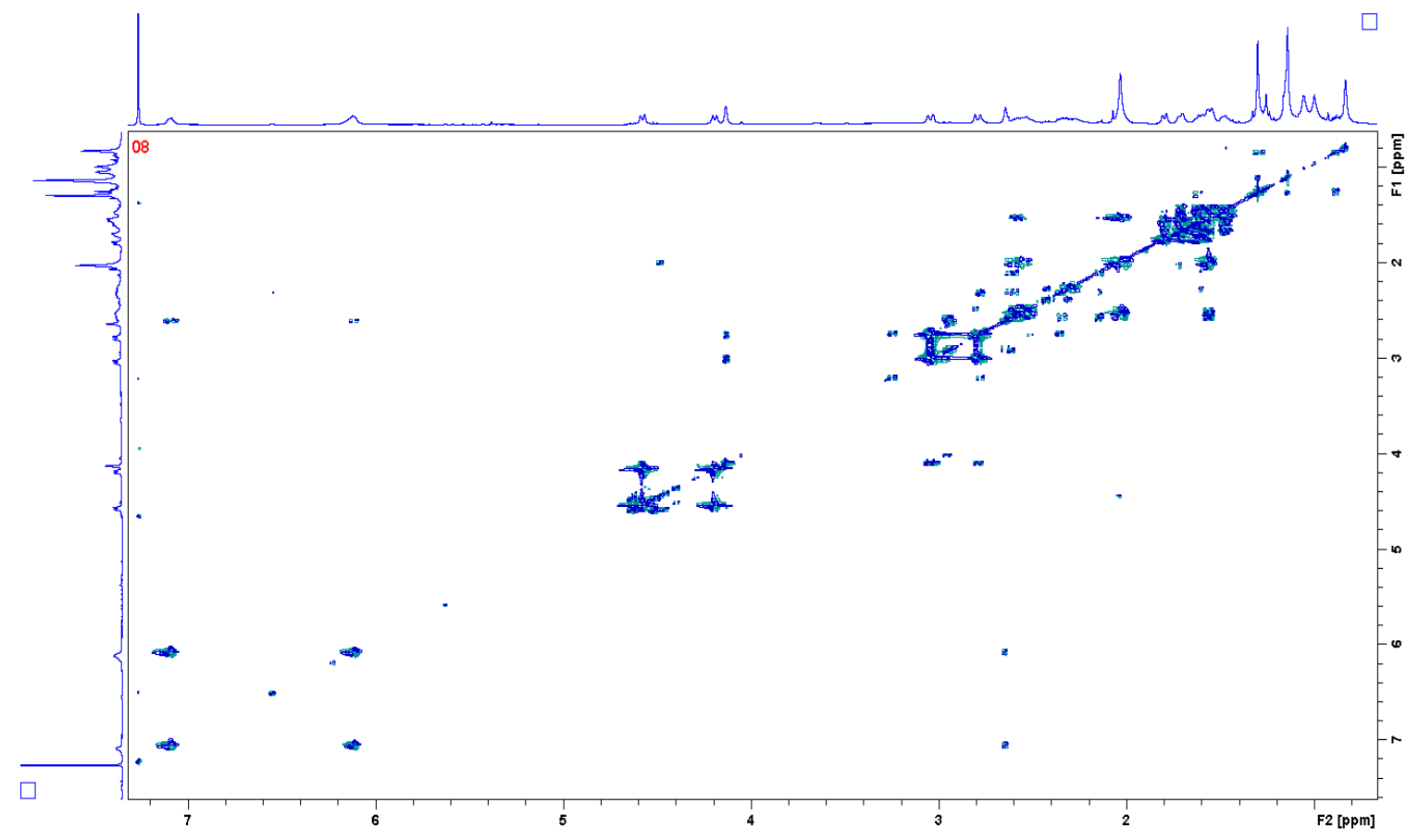

Figure S156. ROESY spectrum of picraviane I (8) in $\mathrm{CDCl}_{3}(600 \mathrm{MHz})$.

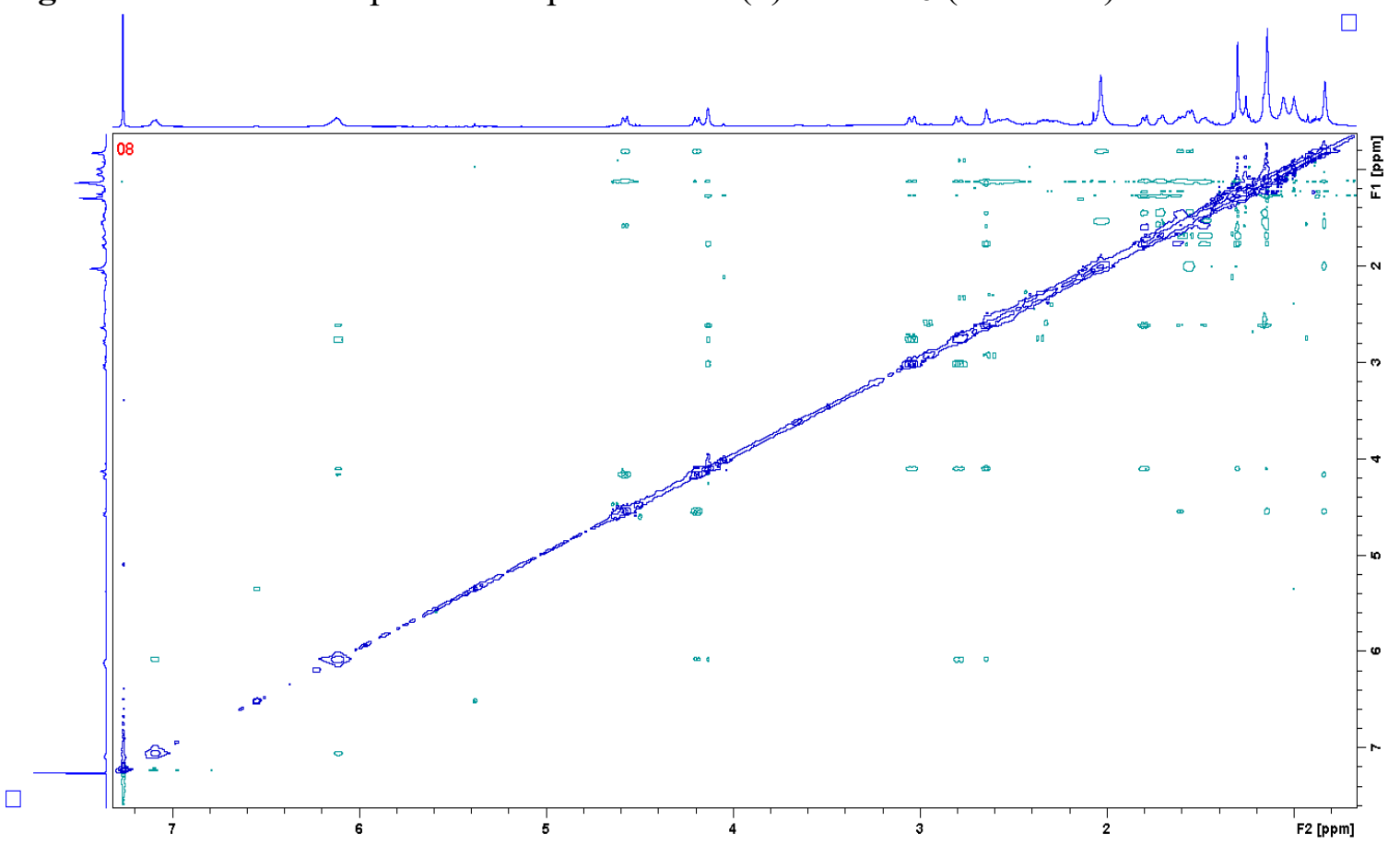


Figure S157. ${ }^{1} \mathrm{H}$ NMR spectrum of picraviane Q (17) in $\mathrm{CDCl}_{3}(600 \mathrm{MHz})$.

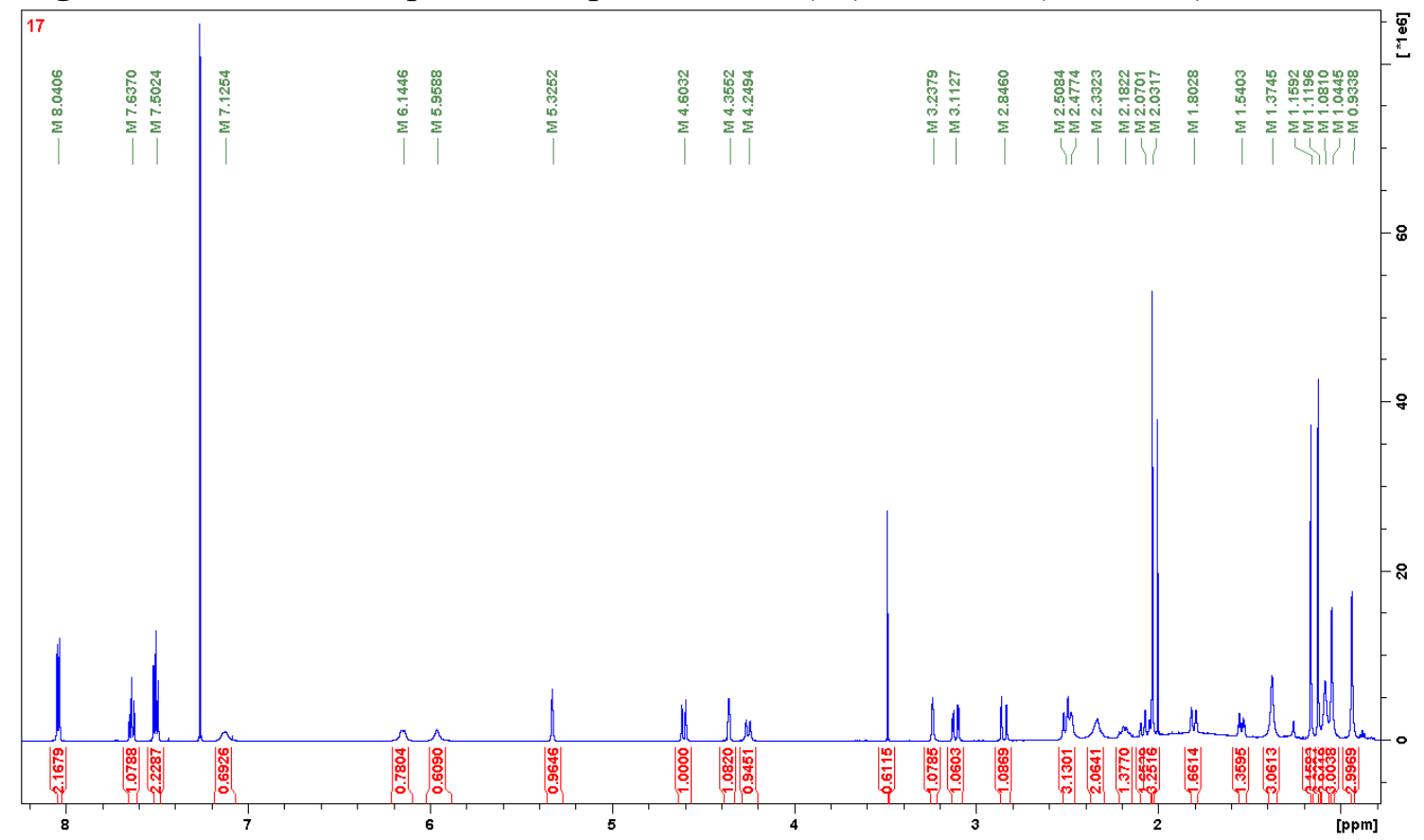

Figure S158. Expansion of the ${ }^{1} \mathrm{H}$ NMR spectrum of picraviane Q (17) in $\mathrm{CDCl}_{3}(600 \mathrm{MHz})$.

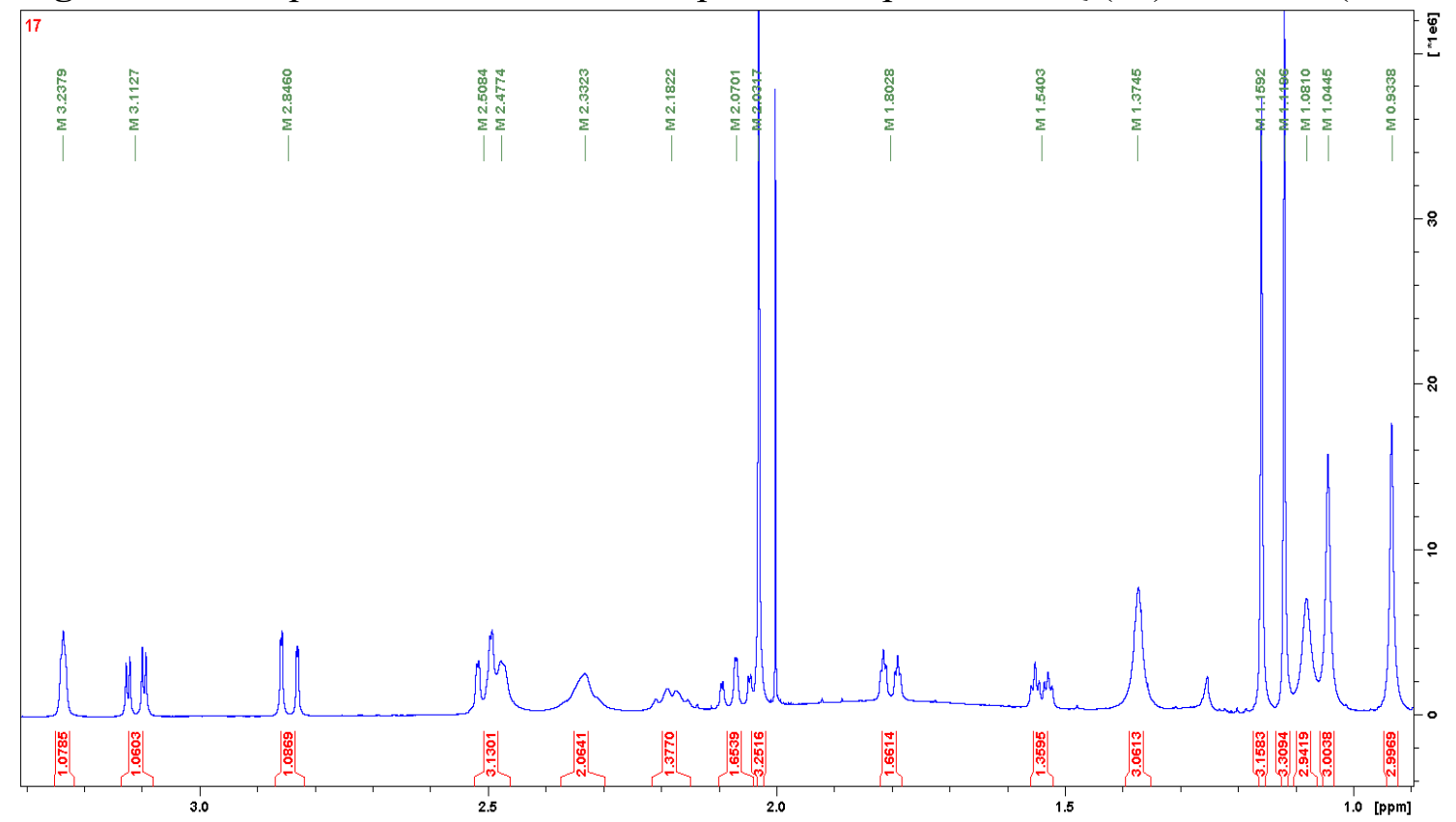


Figure S159. ${ }^{13} \mathrm{C}$ NMR spectrum of picraviane Q (17) in $\mathrm{CDCl}_{3}(150 \mathrm{MHz})$.

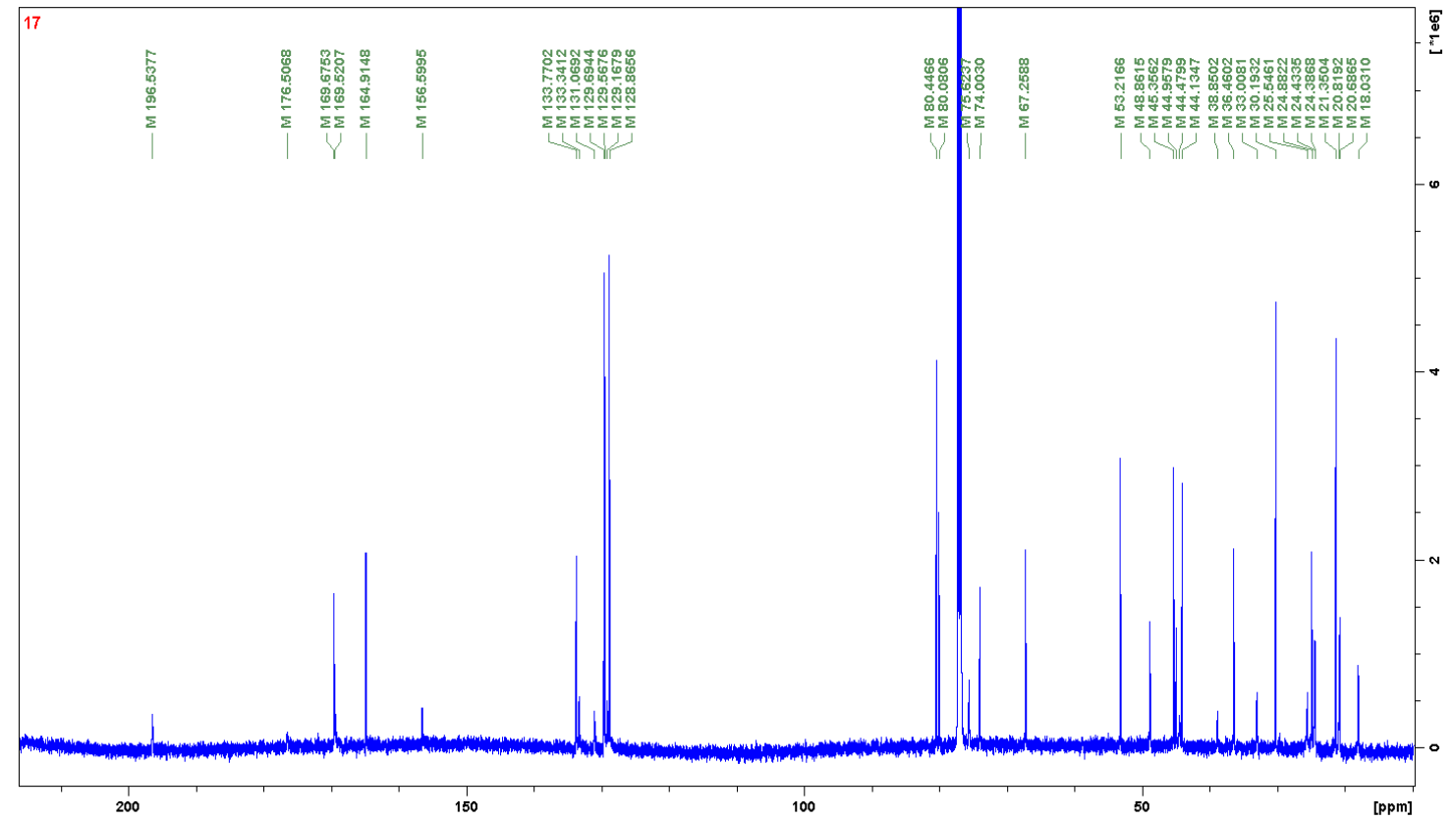

Figure S160. HSQC spectrum of picraviane Q (17) in $\mathrm{CDCl}_{3}(600 \mathrm{MHz})$.

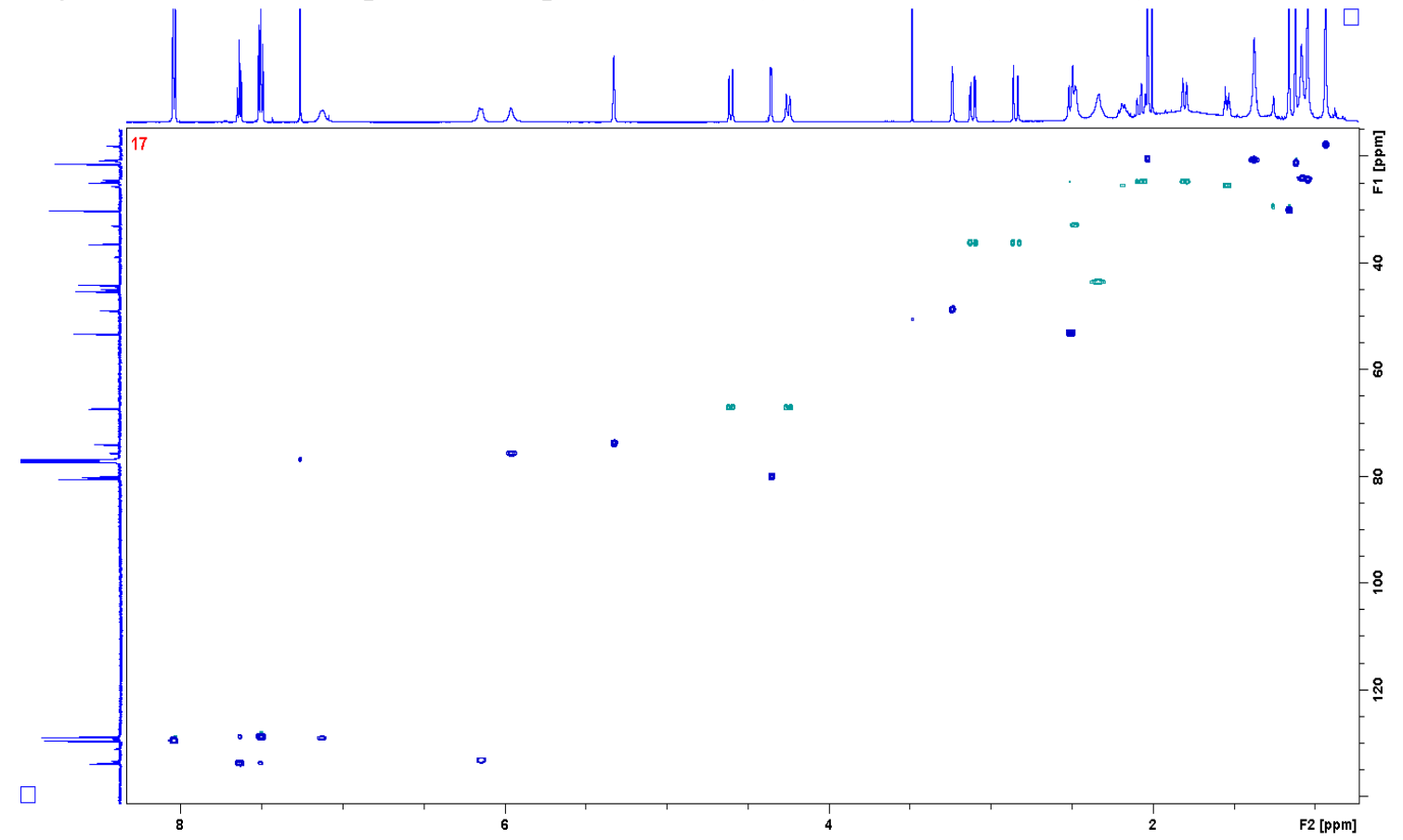


Figure S161. HMBC spectrum of picraviane Q (17) in $\mathrm{CDCl}_{3}(600 \mathrm{MHz})$.

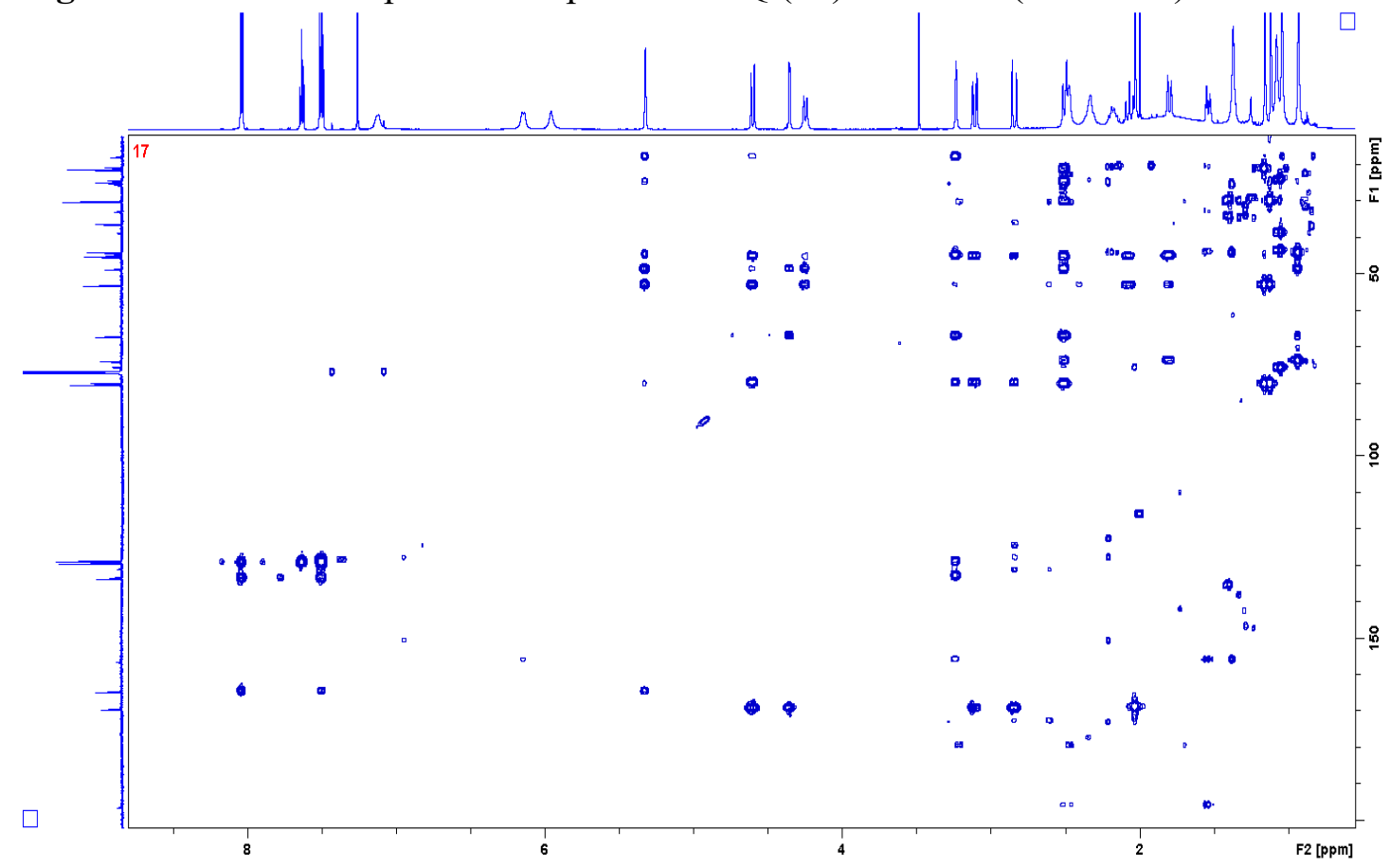

Figure S162. COSY spectrum of picraviane Q (17) in $\mathrm{CDCl}_{3}(600 \mathrm{MHz})$.

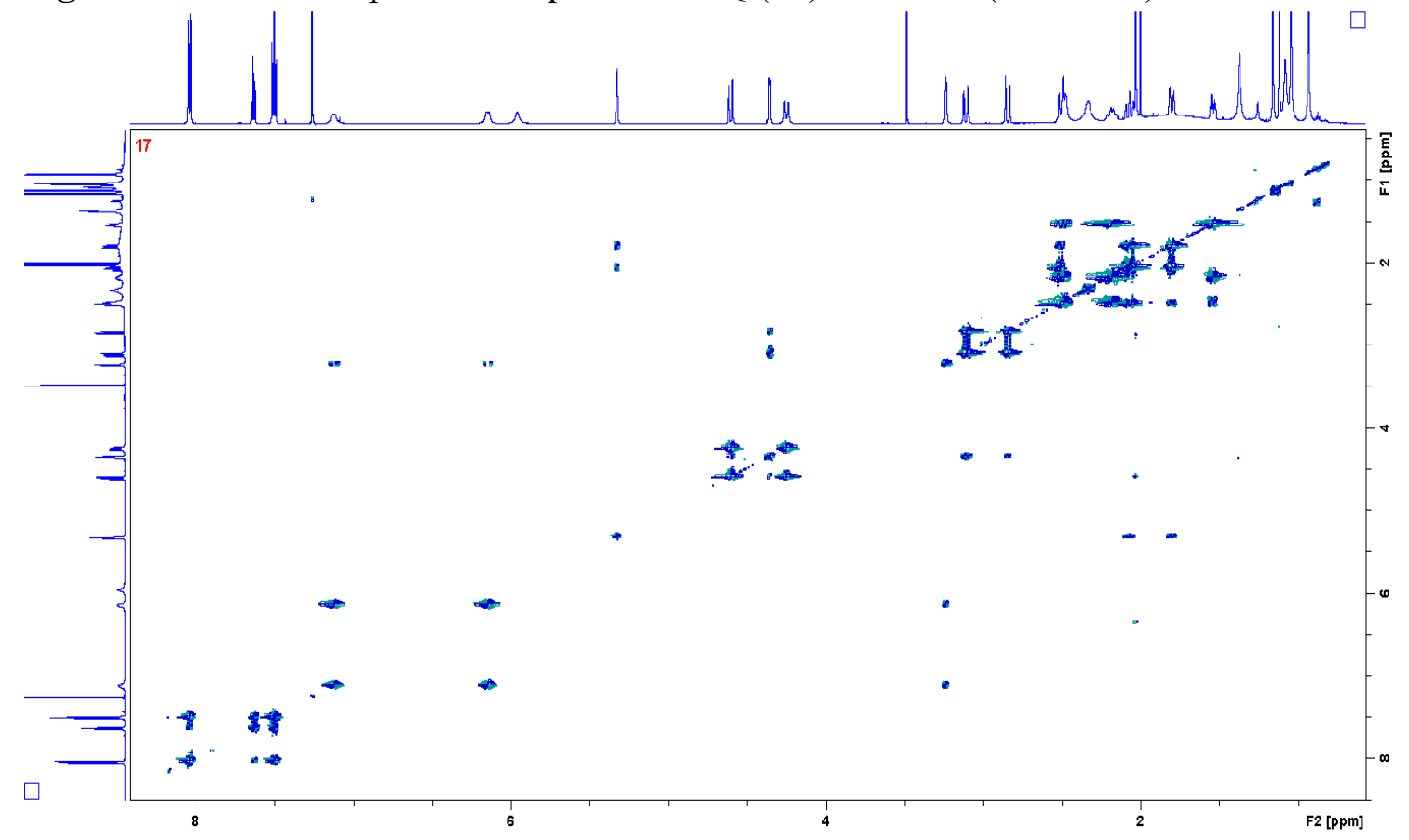


Figure S163. NOESY spectrum of picraviane Q (17) in $\mathrm{CDCl}_{3}(600 \mathrm{MHz})$.

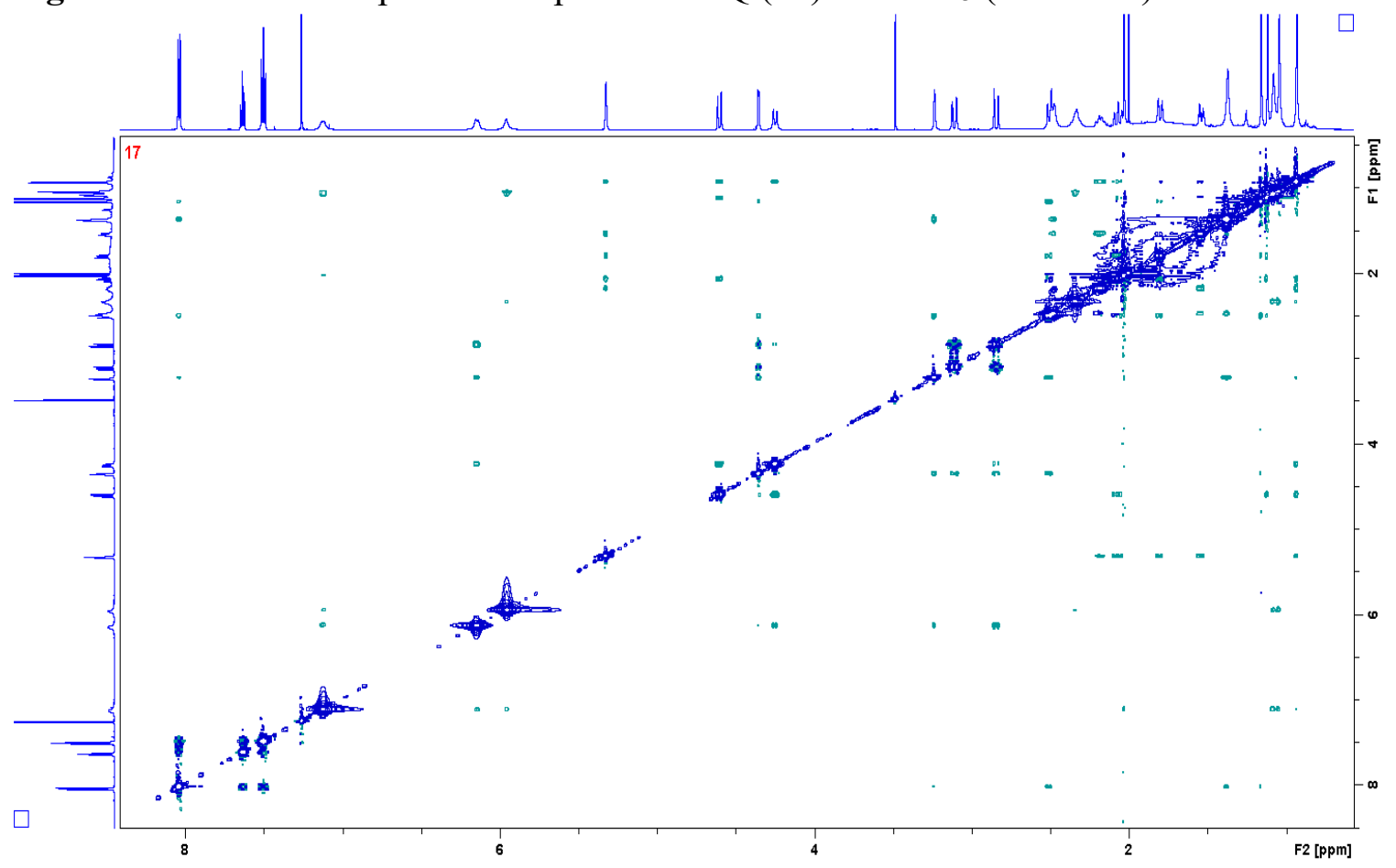

97 
Figure S164. Structures of the five lowest-energy conformers of $(1 S, 5 R, 7 R, 8 R, 9 S, 10 R, 14 R, 19 S)-\mathbf{1 7}$ at the B3LYP/PCM(MeOH)/TZVP level.

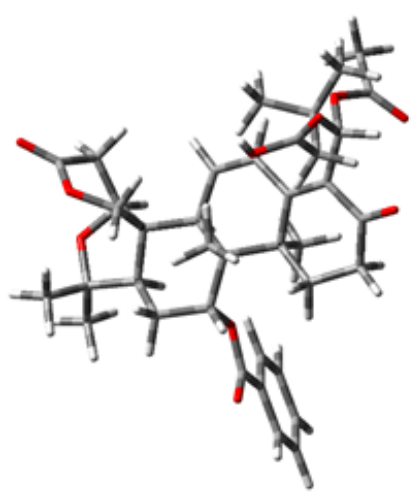

Conf. 1, $0.0 \mathrm{kcal}^{-1}(28 \%)$

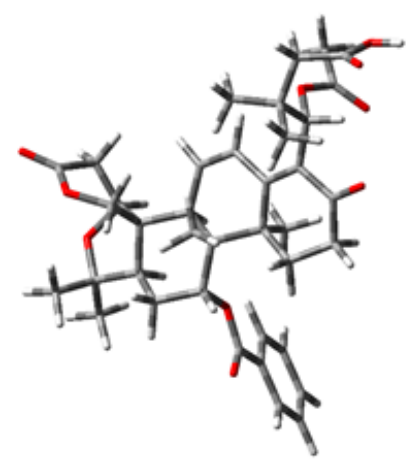

Conf. $3,+0.2 \mathrm{kcal}^{-1}(18 \%)$

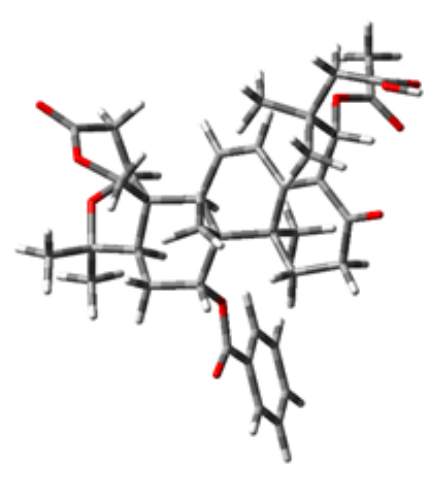

Conf. $2,+0.1 \mathrm{kcal}^{-1}(24 \%)$

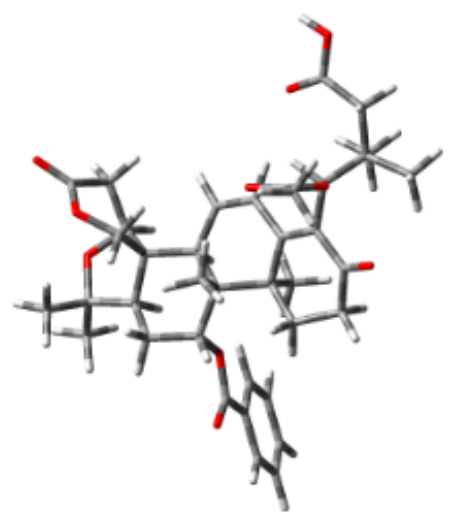

Conf. $4,+0.3 \mathrm{kcal}^{-1}(17 \%)$

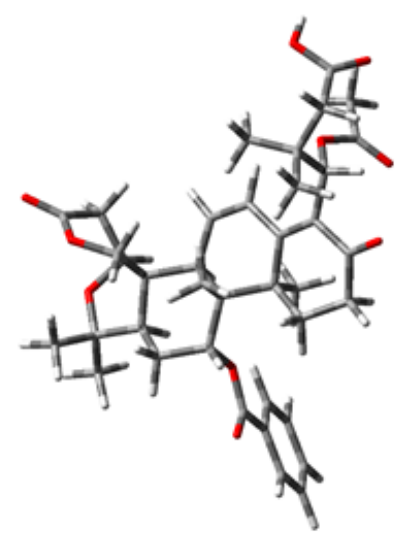

Conf. $5,+0.7 \mathrm{kcal}^{-1}(09 \%)$ 
Figure S165. Comparison of the experimental and calculated UV and ECD spectra of $(1 S, 5 R, 7 R, 8 R, 9 S, 10 R, 14 R, 19 S)-17$ (blue trace) and its enantiomer (red trace). Experimental spectra were acquired in methanol, and UV and ECD spectra were calculated at the [CAM$\mathrm{B} 3 \mathrm{LYP} / \mathrm{PCM}(\mathrm{MeOH}) / \mathrm{TZVP}$ level for the Boltzmann average of the six lowest-energy conformers identified for $(1 S, 5 R, 7 R, 8 R, 9 S, 10 R, 14 R, 19 S)-17$.

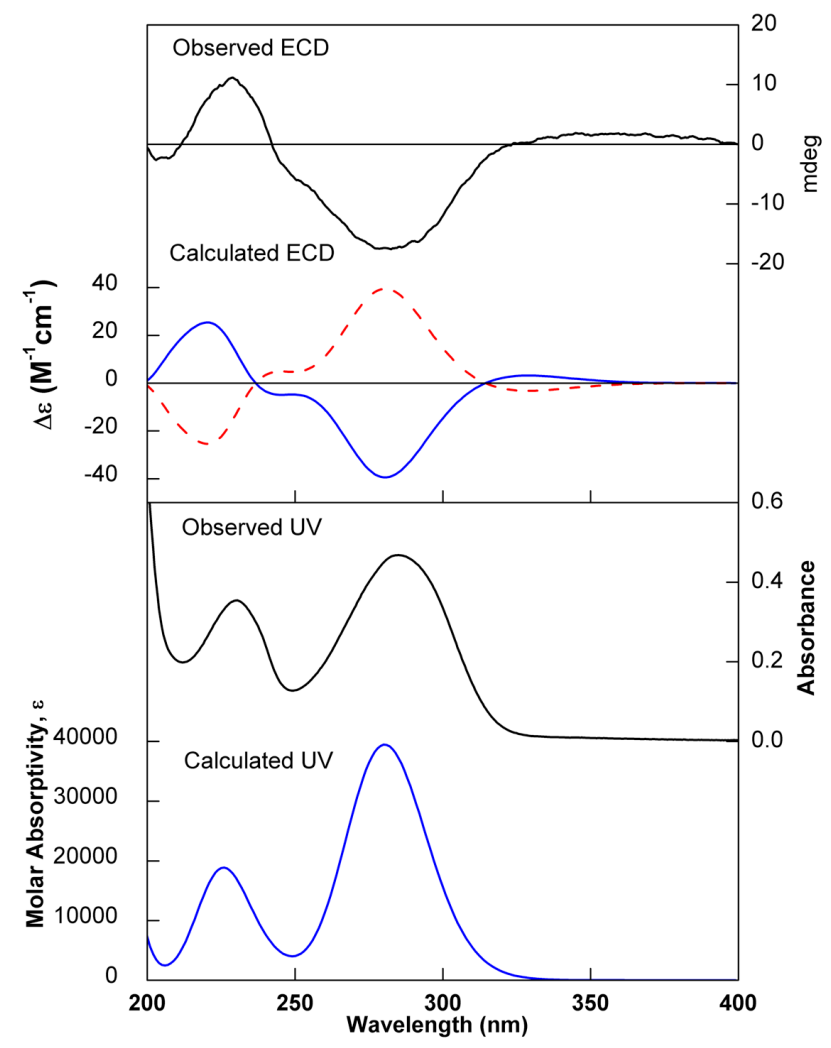

Figure S166. Calculated ECD spectrum of the epimer $(1 S, 5 R, 7 R, 8 R, 9 S, 10 R, 14 R, 19 R)-17$ at the CAM-B3LYP/PCM(MeOH)/TZVP level.

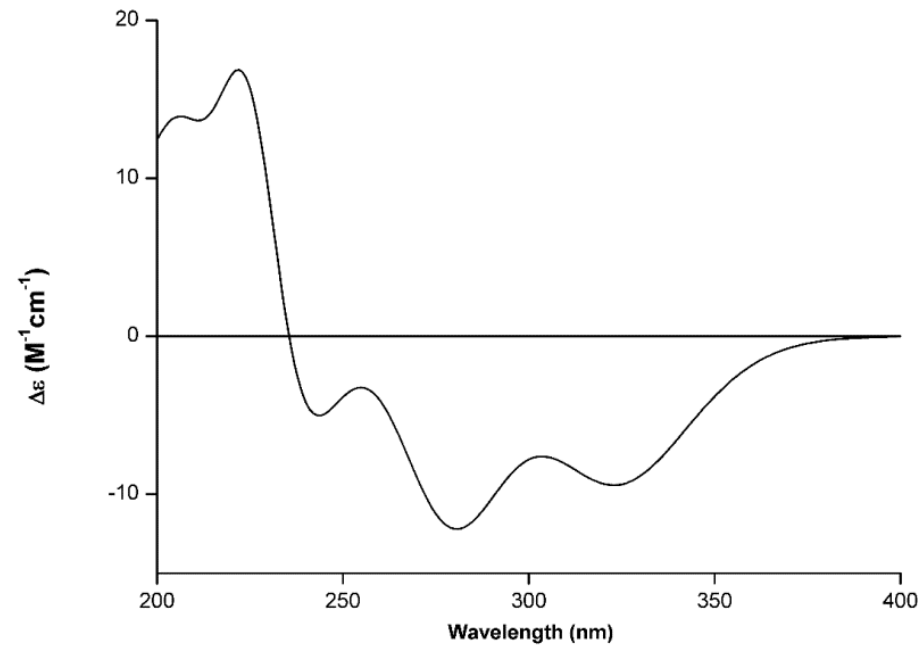


Figure S167. ${ }^{1} \mathrm{H}$ NMR spectrum of picraviane $\mathrm{P}(\mathbf{1 6})$ in $\mathrm{CDCl}_{3}(600 \mathrm{MHz})$.

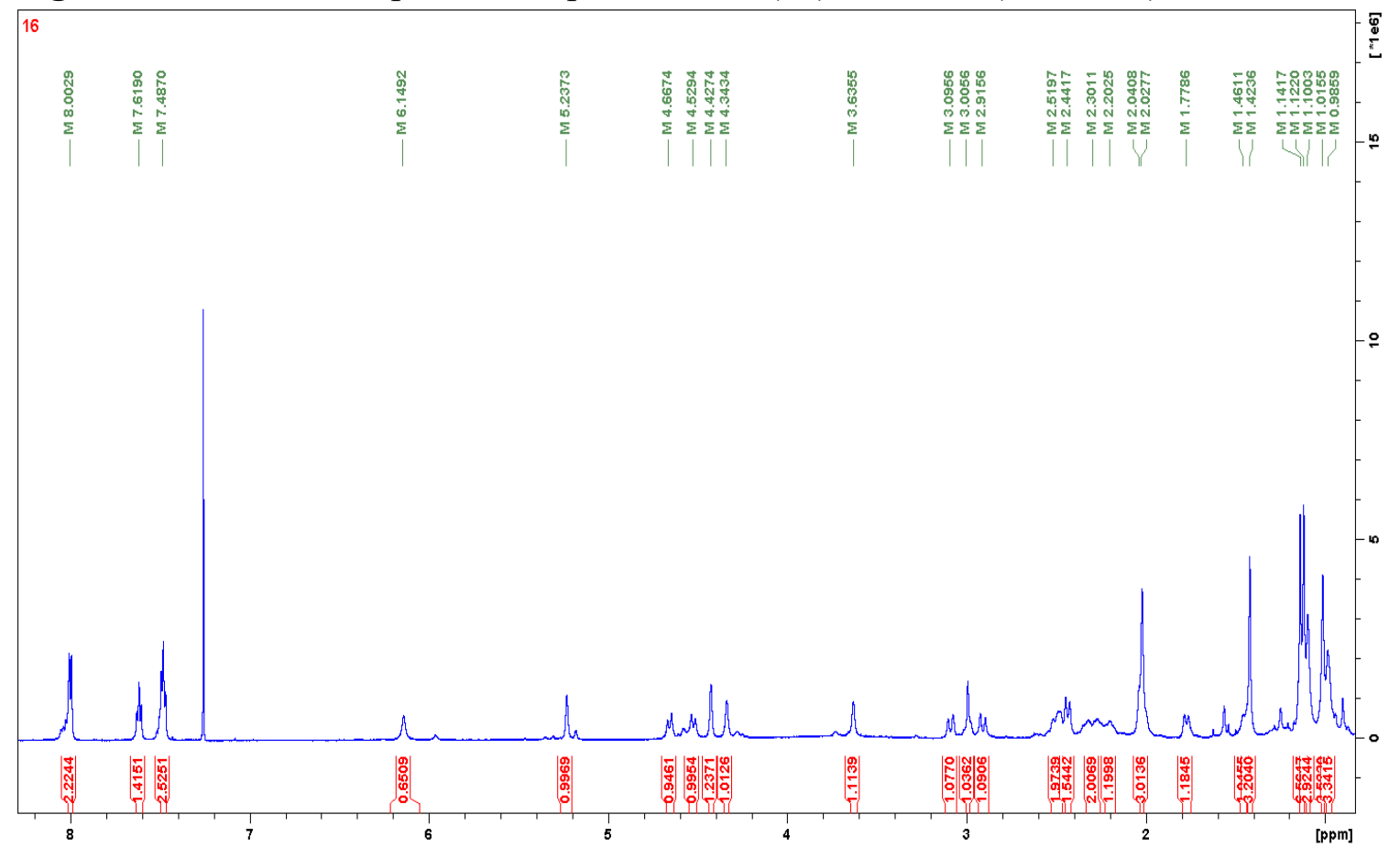

Figure S168. Expansion of the ${ }^{1} \mathrm{H}$ NMR spectrum of picraviane $\mathrm{P}(16)$ in $\mathrm{CDCl}_{3}(600 \mathrm{MHz})$.

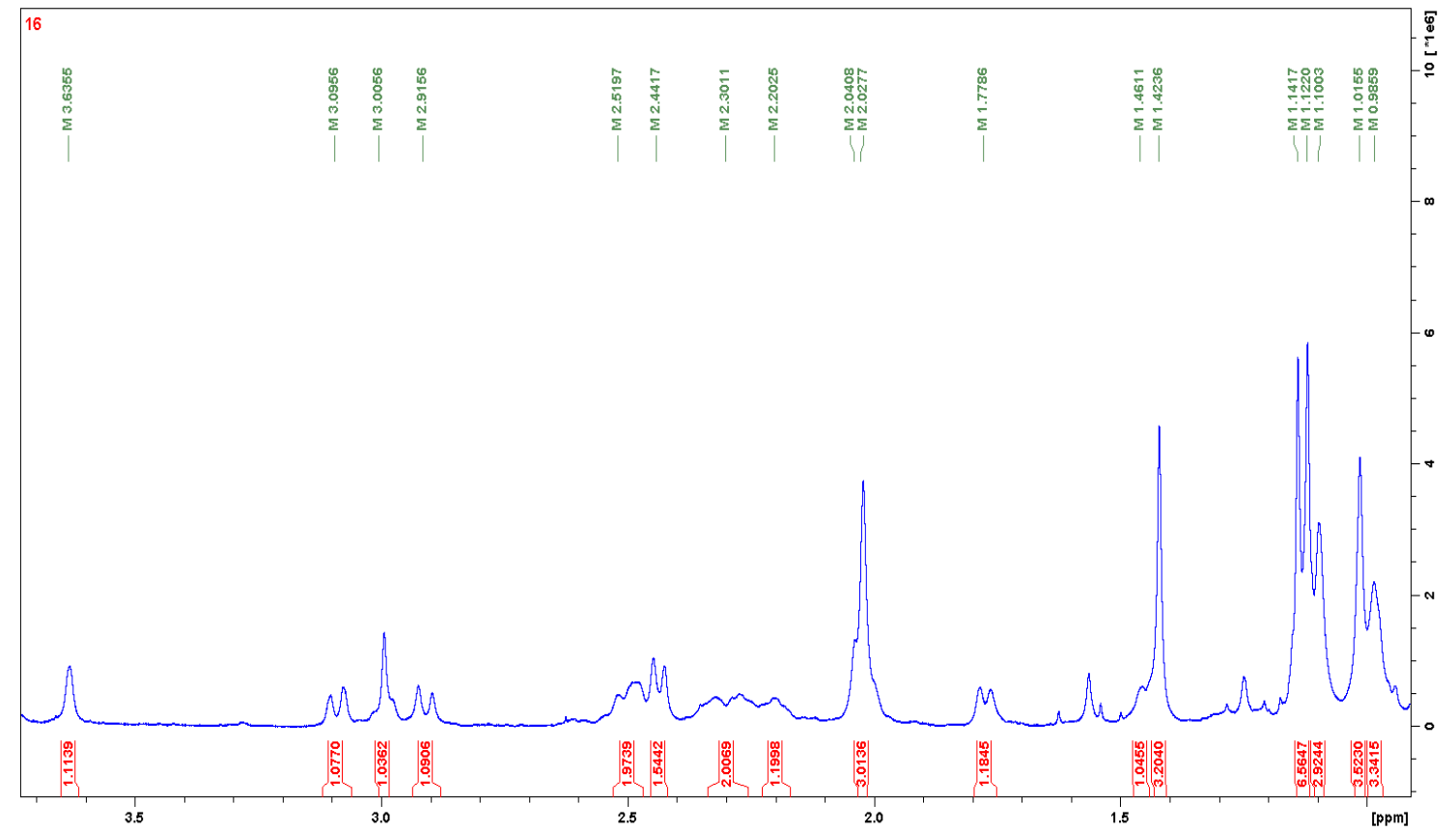


Figure S169. ${ }^{13} \mathrm{C}$ NMR spectrum of picraviane $\mathrm{P}(\mathbf{1 6})$ in $\mathrm{CDCl}_{3}(150 \mathrm{MHz})$.

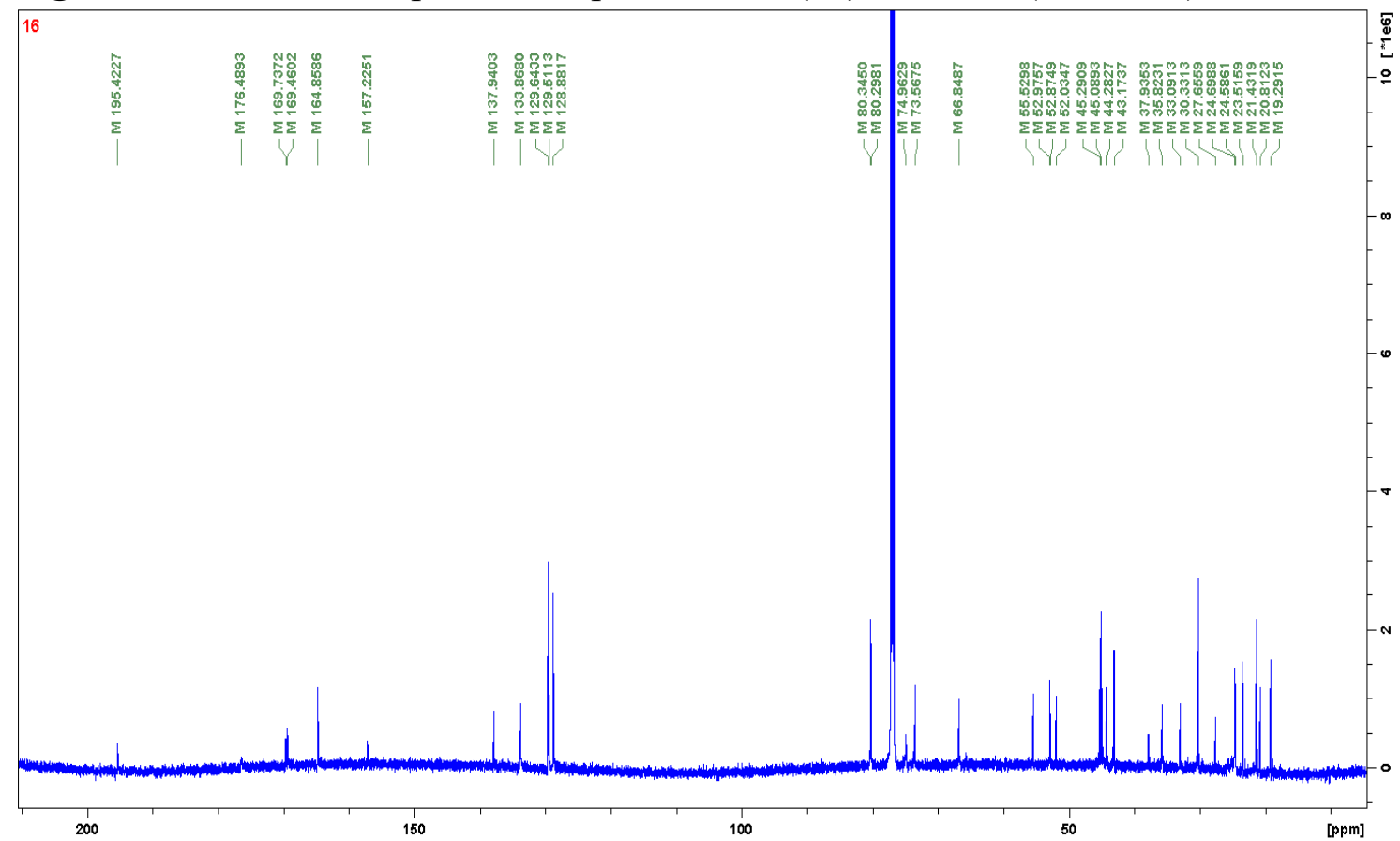

Figure S170. HSQC spectrum of picraviane $\mathrm{P}(\mathbf{1 6})$ in $\mathrm{CDCl}_{3}(600 \mathrm{MHz})$.

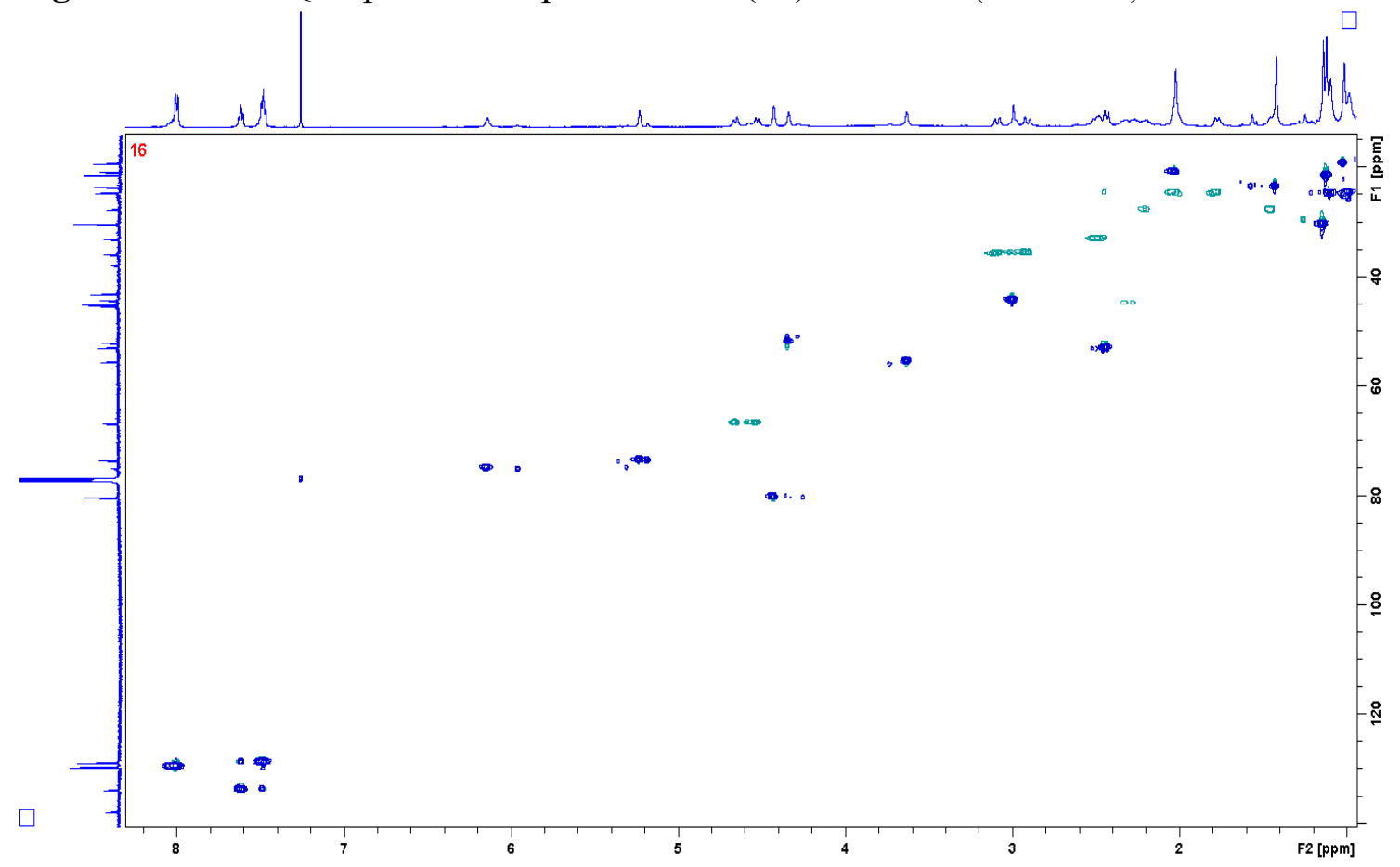


Figure S171. HMBC spectrum of picraviane $\mathrm{P}(\mathbf{1 6})$ in $\mathrm{CDCl}_{3}(600 \mathrm{MHz})$.

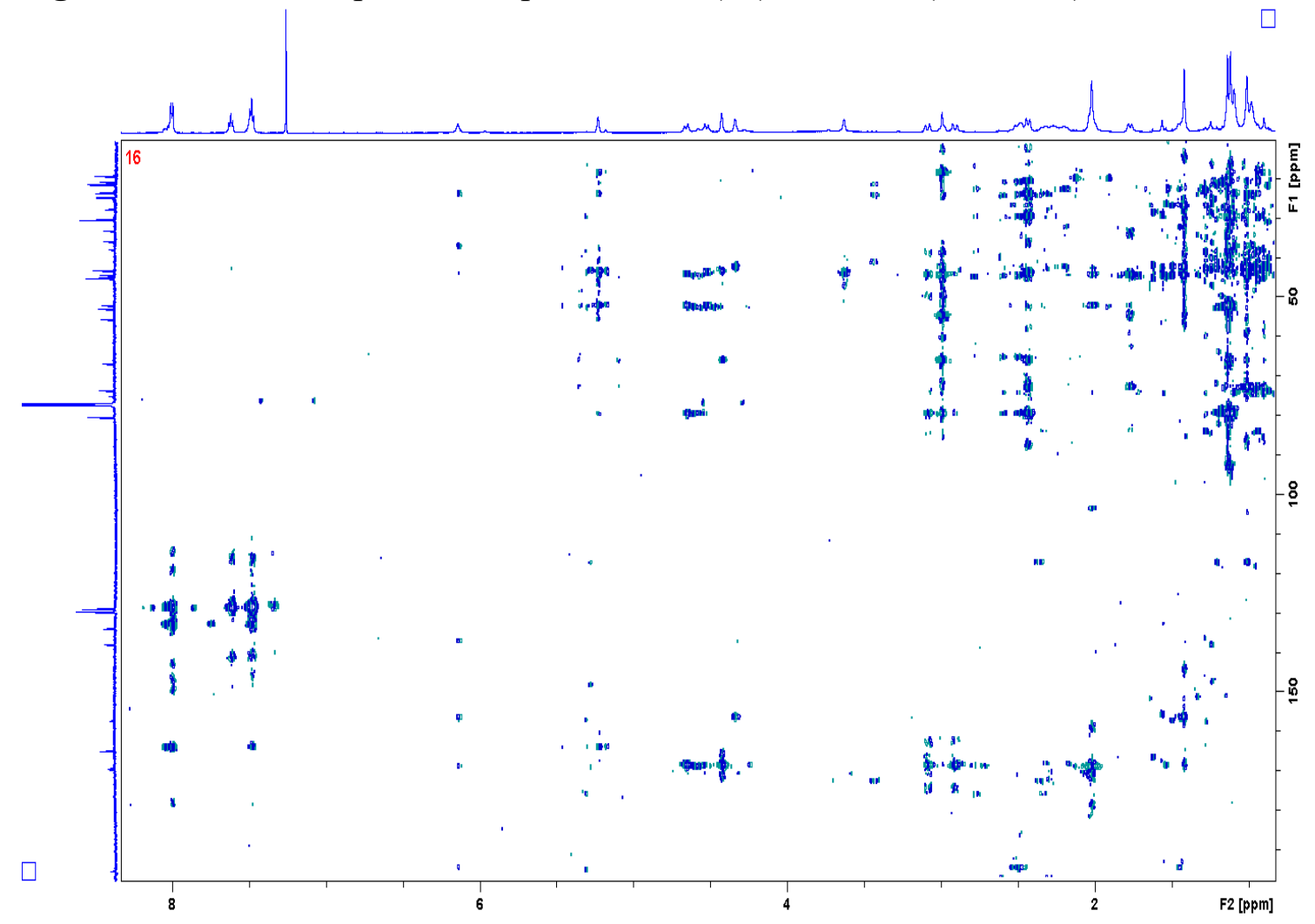

Figure S172. COSY spectrum of picraviane $\mathrm{P}(\mathbf{1 6})$ in $\mathrm{CDCl}_{3}(600 \mathrm{MHz})$.

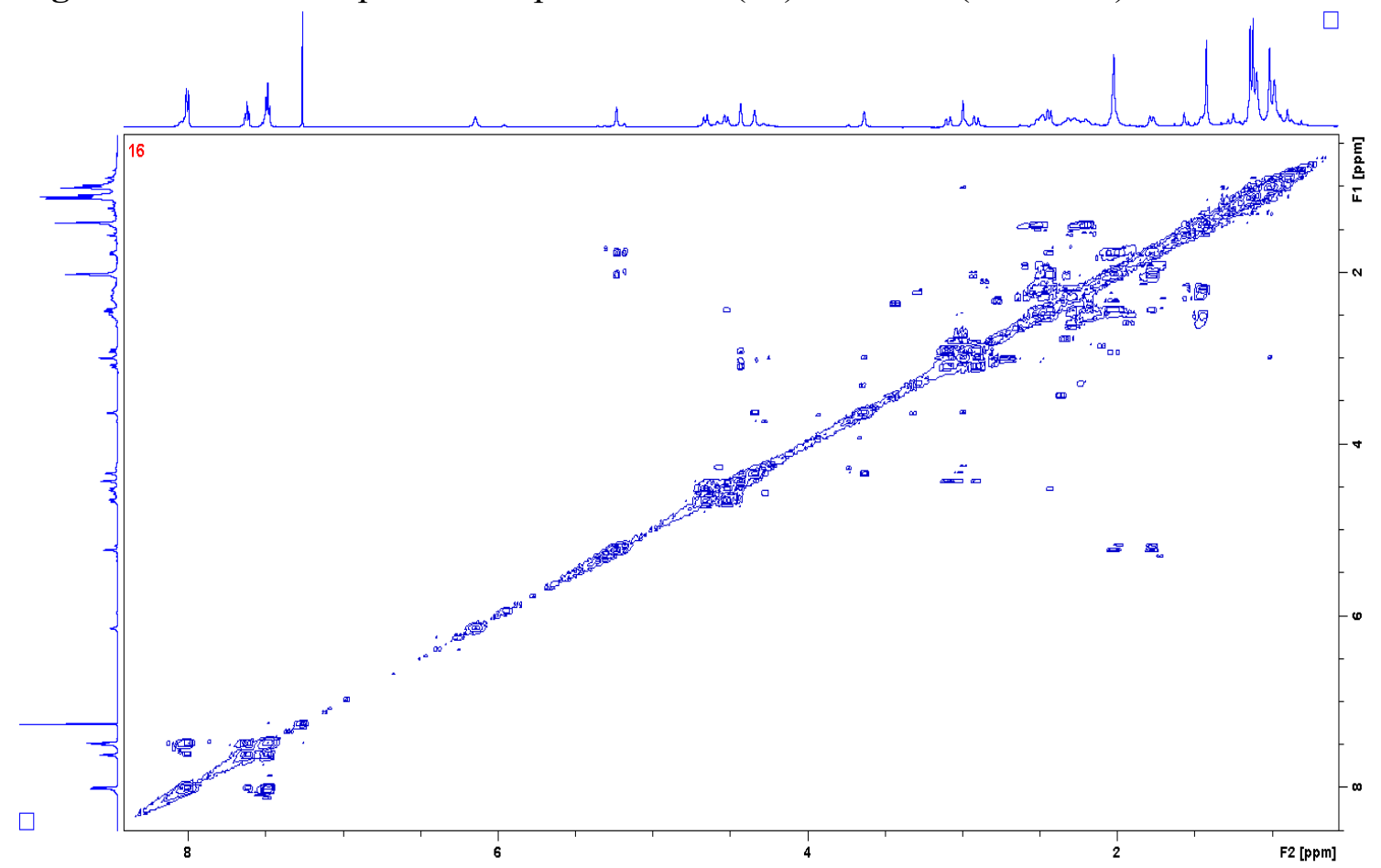


Figure S173. ROESY spectrum of picraviane $\mathrm{P}(16)$ in $\mathrm{CDCl}_{3}(600 \mathrm{MHz})$.

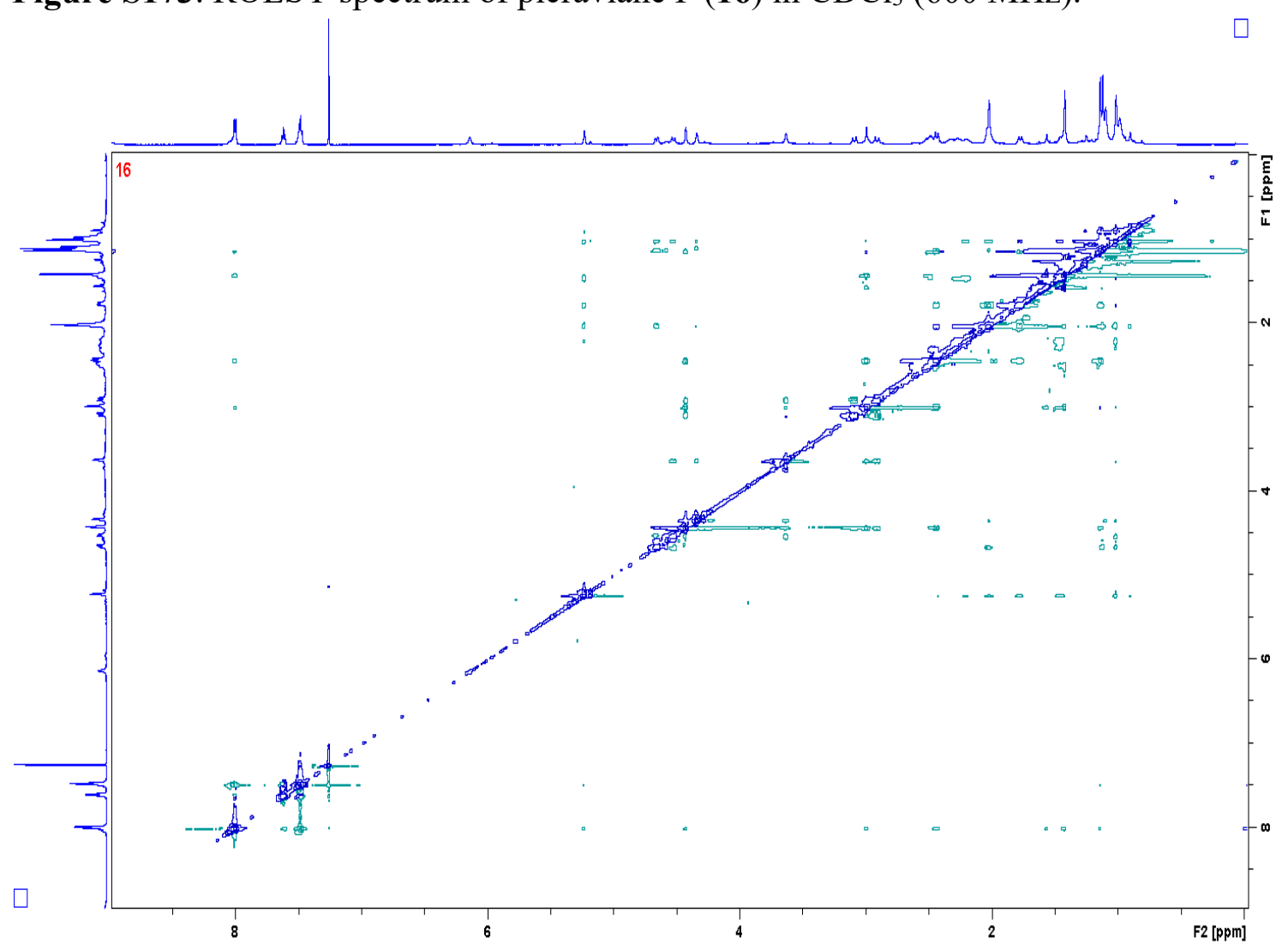

103 
Figure S174. Effect of compounds 6, 10, 14, 16, 17, 18, 19 and 21 tested in different concentrations $(1.56-100 \mu \mathrm{M})$ on the viability of MDA-MB-231 triple negative breast cancer cells. $^{a}$ Data represent mean $\pm \mathrm{SD}$ of three independent assays in triplicate. Control indicates the cell viability in the absence of the tested compounds.

Compound 6:

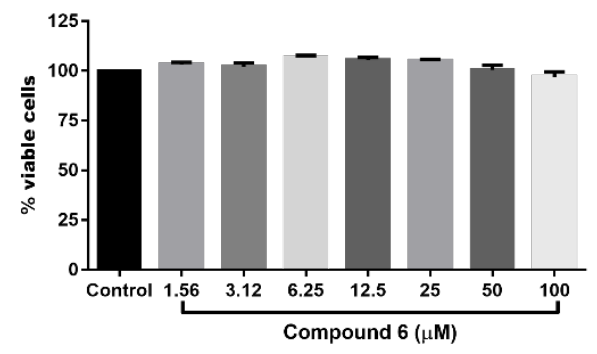

Compound 14:

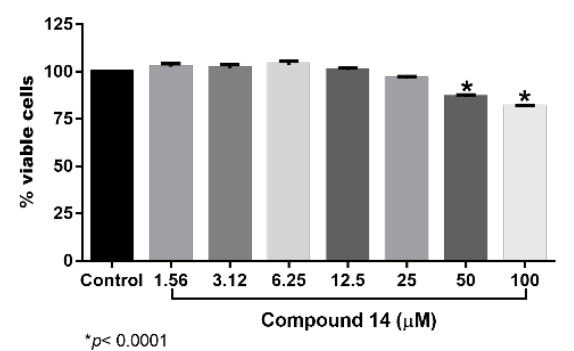

Compound 17:

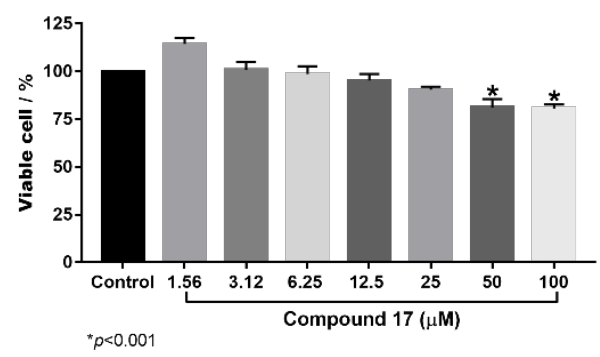

Compound 19:

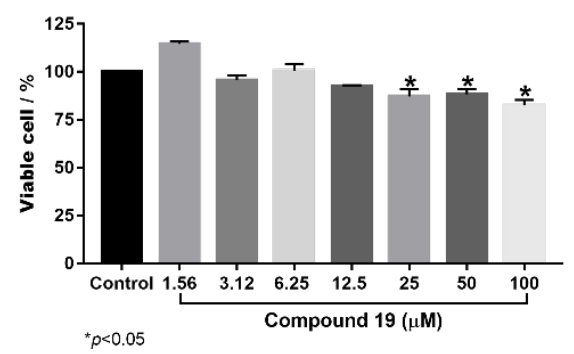

Compound 10:

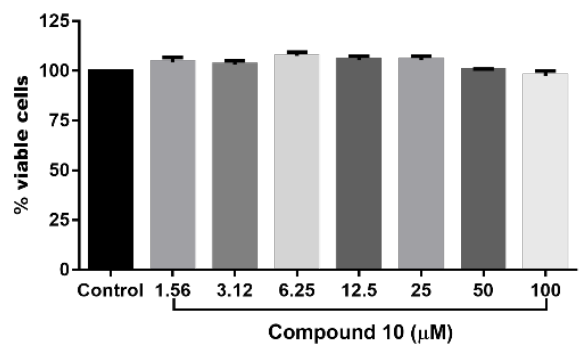

Compound 16:

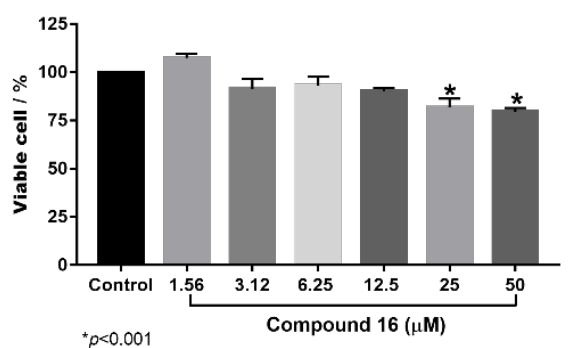

Compound 18:

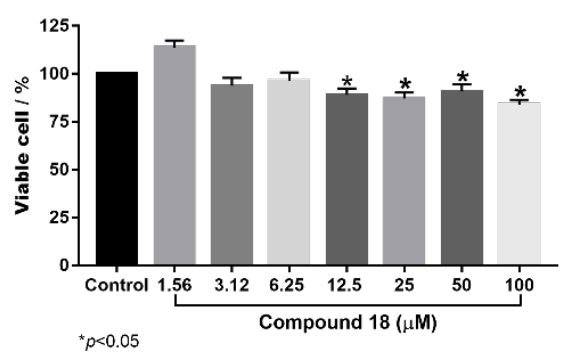

Compound 21:

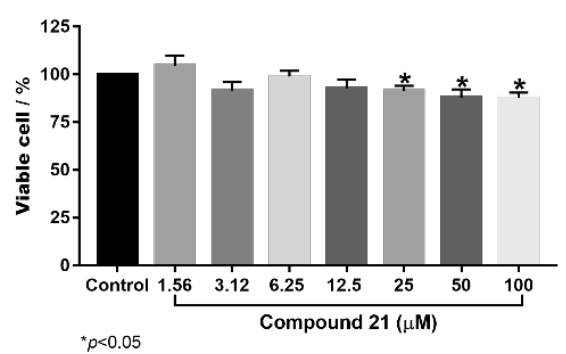

${ }^{\text {a }} \mathrm{IC}_{50}$ could not be calculated due the low activity of the compounds. 
Figure S175. Effect of compounds 6, 10, 14, 16, 17, 18, 19 and 21 tested in different concentrations $(1.56-100 \mu \mathrm{M})$ on the viability of SKBR-31 Her2-overexpressing breast cancer cells. ${ }^{a}$ Data represent mean \pm SD of three independent assays in triplicate. Control indicates the cell viability in the absence of the tested compounds.

Compound 6:

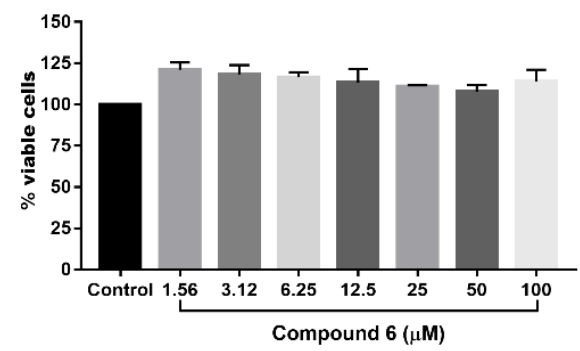

Compound 14:

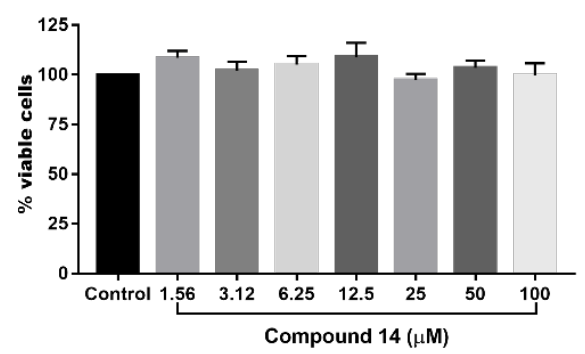

Compound 17:

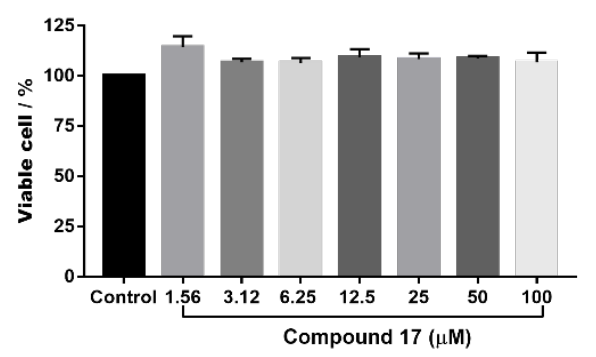

Compound 19:

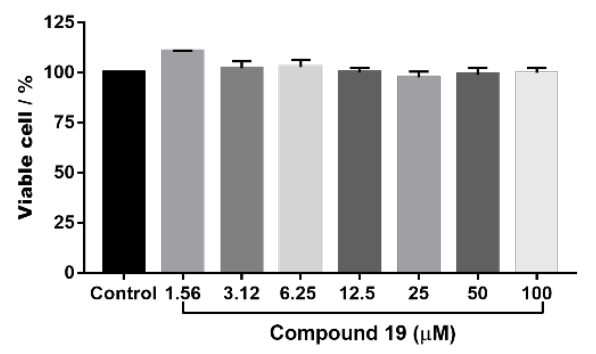

Compound 10:

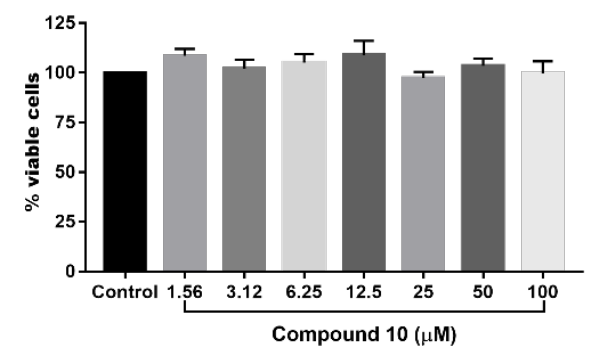

Compound 16:

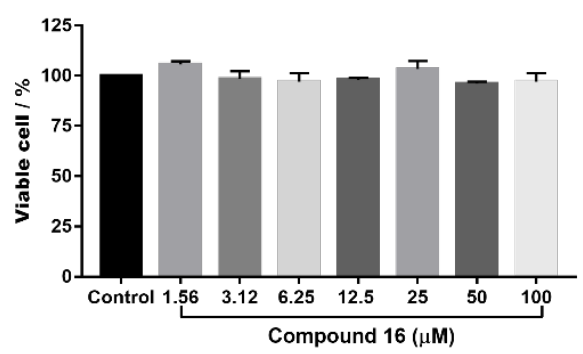

Compound 18:

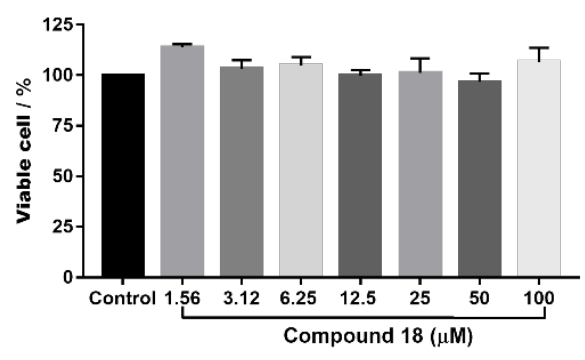

Compound 21:

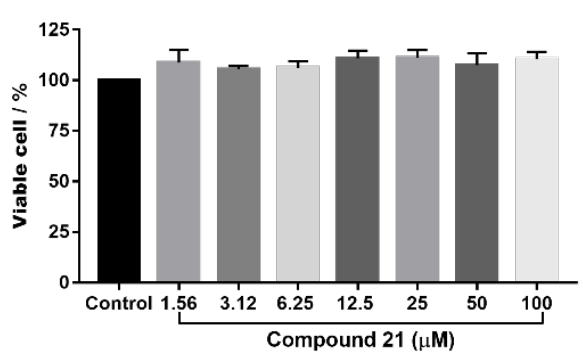

${ }^{a} \mathrm{IC}_{50}$ could not be calculated due the low activity of the compounds. 
Figure S176. Effect of compounds 6, 10, 14, 16, 17, 18, 19 and 21 tested in different concentrations $(1.56-100 \mu \mathrm{M})$ on the viability of A549 lung tumor cells. ${ }^{a}$ Data represent mean \pm SD of three independent assays in triplicate. Control indicates the cell viability in the absence of the tested compounds.

Compound 6:

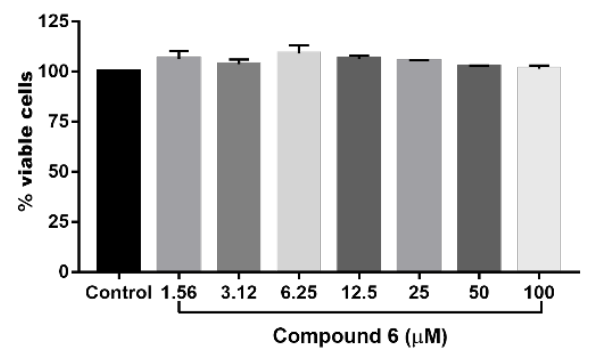

Compound 14:

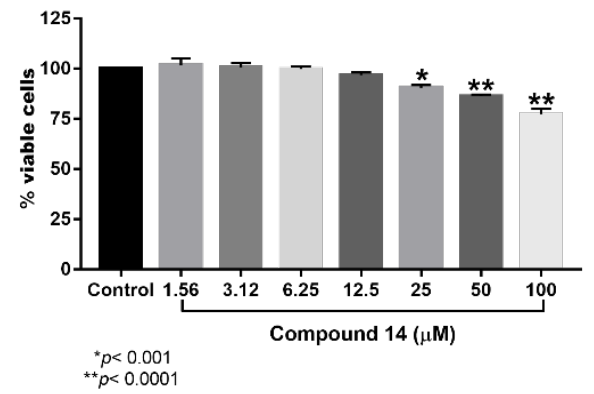

Compound 17:

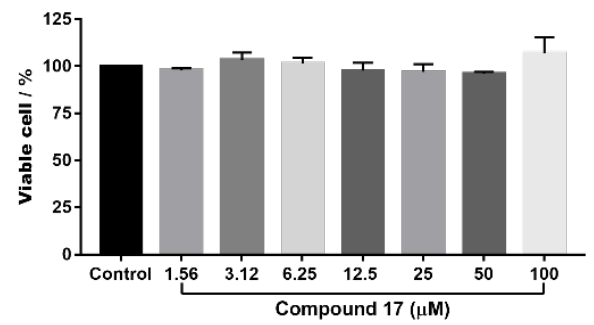

Compound 19:

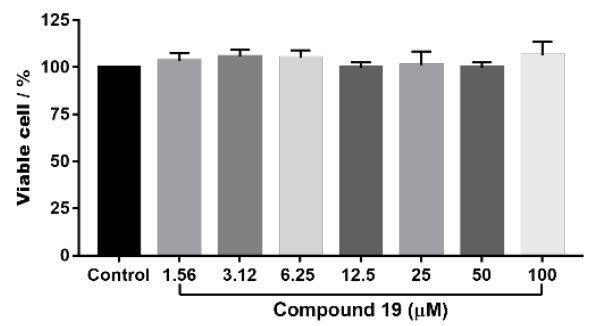

Compound 10:

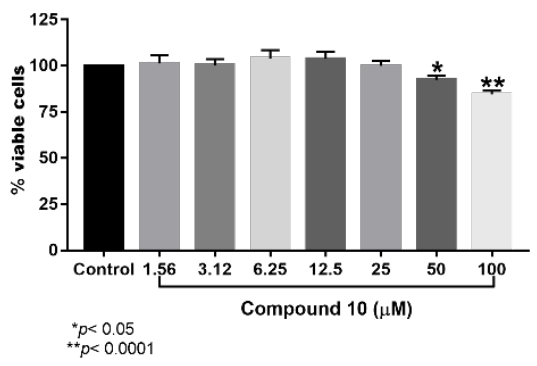

Compound 16:

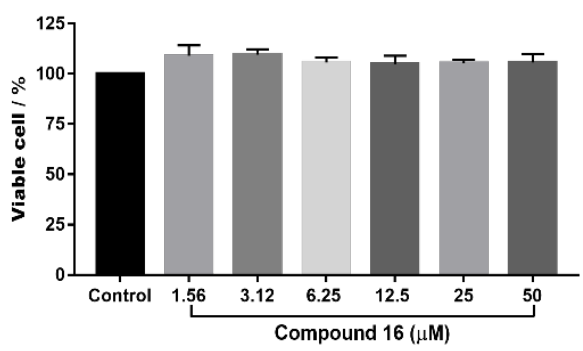

Compound 18:

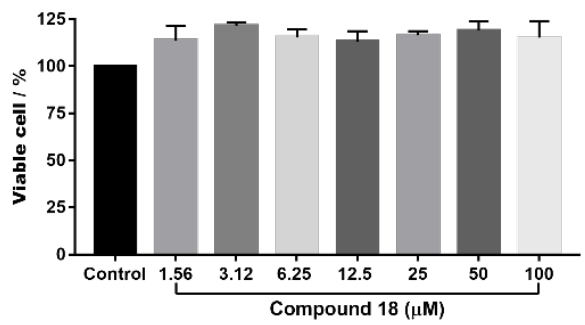

Compound 21:

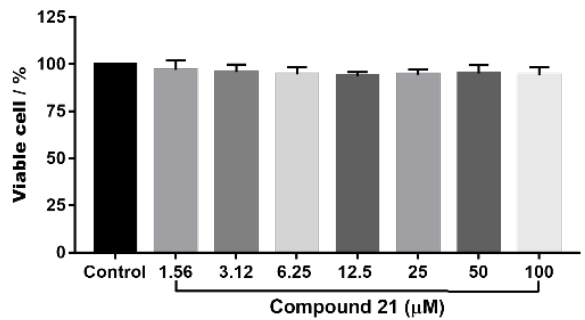

${ }^{a} \mathrm{IC}_{50}$ could not be calculated due the low activity of the compounds. 